\title{
Catalyst-controlled Regioselective Chlorination of Phenols and Anilines through a Lewis basic selenoether catalyst
}

\author{
Andrew N. Dinh, Sean M. Maddox, Sagar D. Vaidya, Mirza A. Saputra, Christopher J. \\ Nalbandian and Jeffrey L. Gustafson* \\ Department of Chemistry and Biochemistry at San Diego State University, \\ 5500 Campanile Drive, San Diego, California 92182-1030 (USA)
}

Supporting Information 


\section{Table of Contents}
A. Examples of Crude NMR Spectra for Determination of Regioselectivity ..................S-3

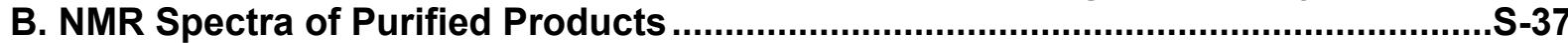
C. Crystal Structure Experimental Summary...........................................................69
D. Isotopically Labeled Experiments with Lewis Base Catalysts ............................... -71
E. Preliminary Mechanistic Experiment: Observing NMR Shifts with Catalyst 10 and $\mathbf{N -}$

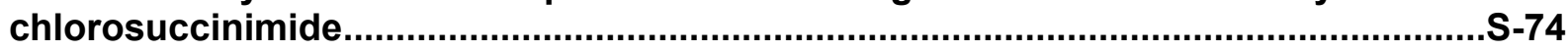
F. Full Optimization Table for ortho chlorination of compound $2 a$............................. -77
G. Measurement of complete conversion with excess NCS .................................... 


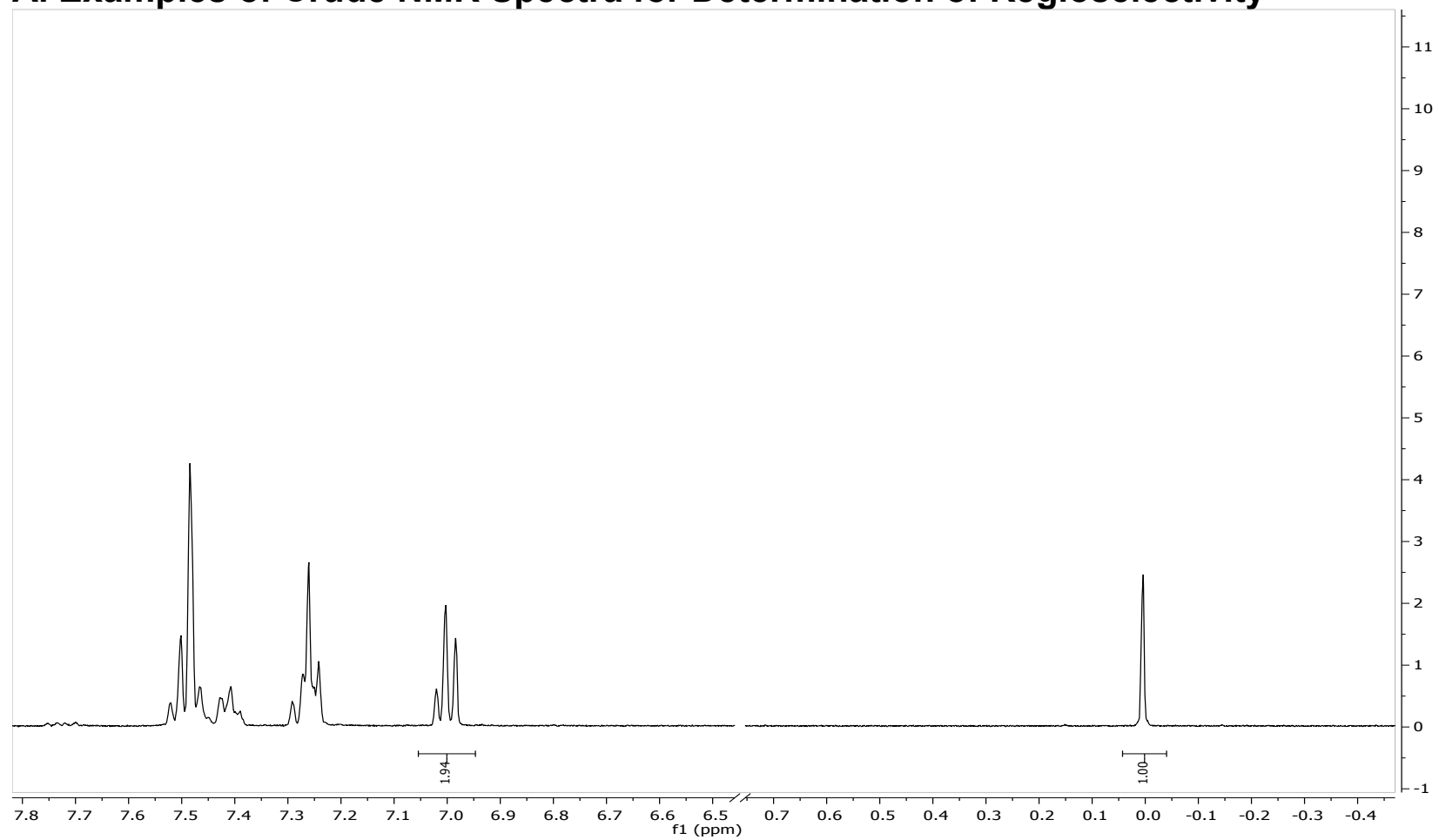

Example of ${ }^{1} \mathrm{H}\left(500 \mathrm{MHz}, \mathrm{CDCl}_{3}\right), 11 \mathrm{a}+$ catalyst 3 (triphenylphosphine sulfide); $\mathrm{t}=0 \mathrm{~h}$

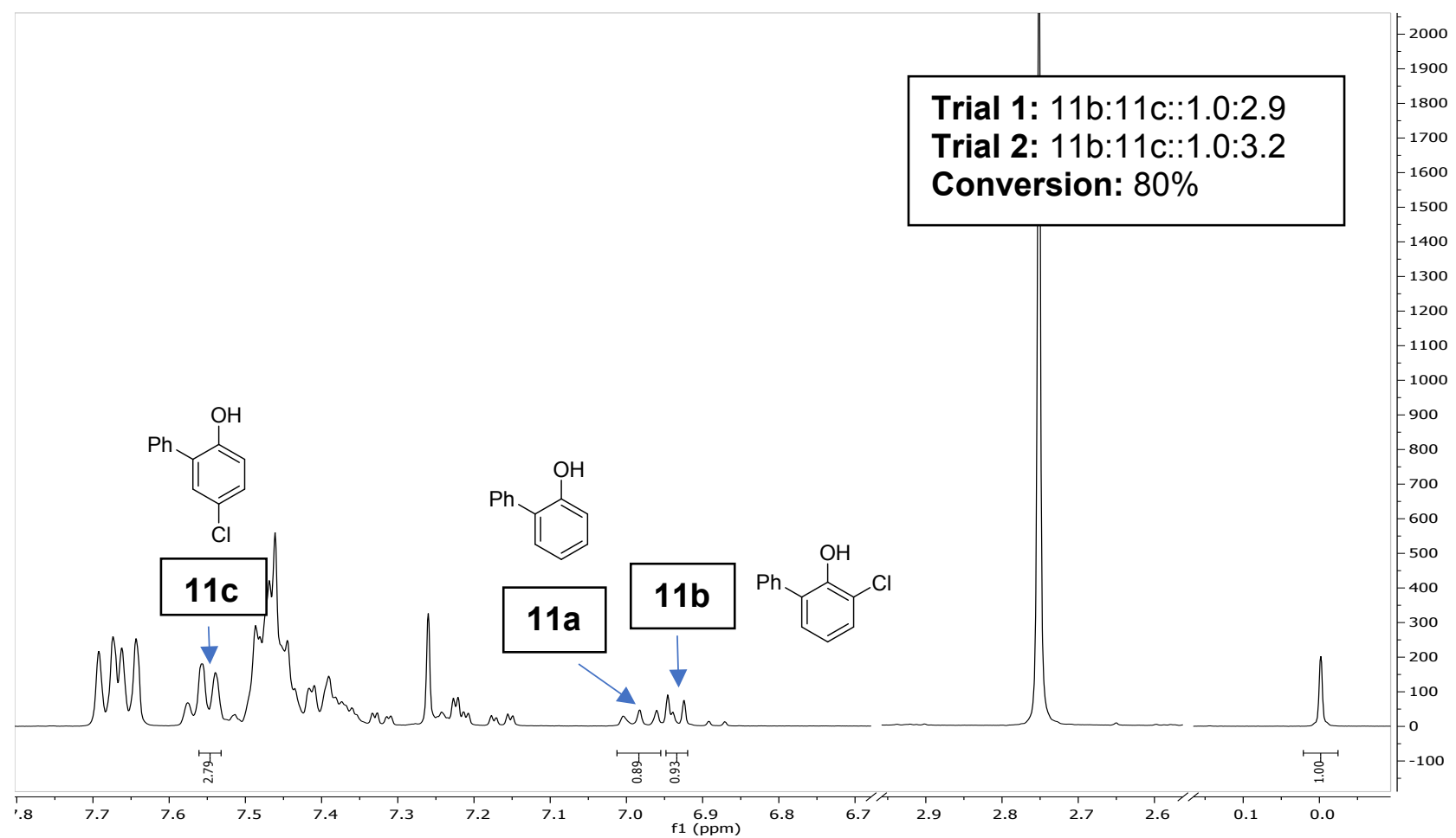

Example of ${ }^{1} \mathrm{H}\left(400 \mathrm{MHz}, \mathrm{CDCl}_{3}\right), 11 \mathrm{a}+$ catalyst 3 (triphenylphosphine sulfide); $\mathrm{t}=12 \mathrm{~h}$. 


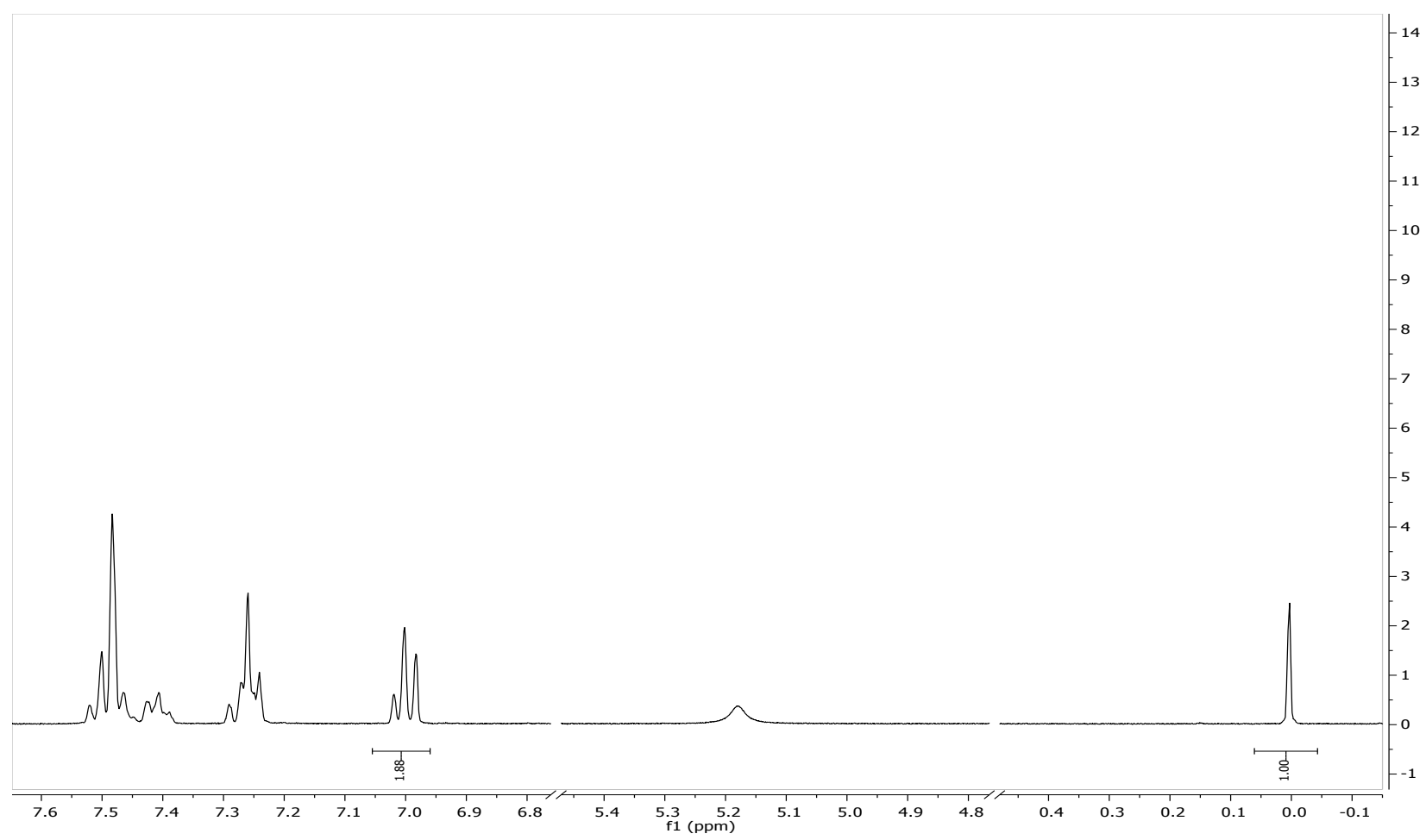

Example of ${ }^{1} \mathrm{H}\left(500 \mathrm{MHz}, \mathrm{CDCl}_{3}\right), 11 \mathrm{a}+$ catalyst $10 ; \mathrm{t}=0 \mathrm{~h}$

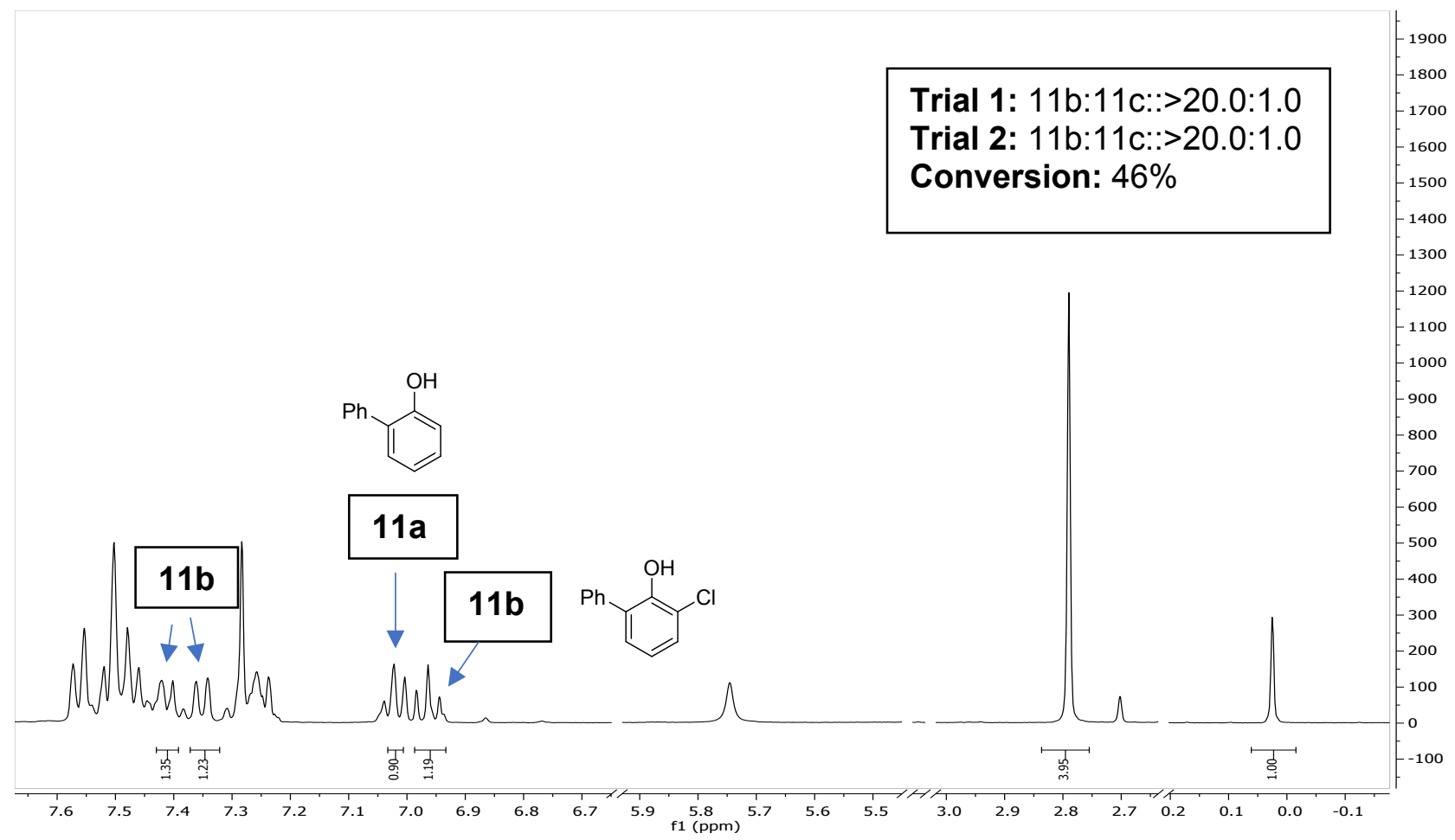

Example of ${ }^{1} \mathrm{H}\left(400 \mathrm{MHz}, \mathrm{CDCl}_{3}\right), 11 \mathrm{a}+$ catalyst 10; Full conversion. 11c is not observed in crude NMR. 


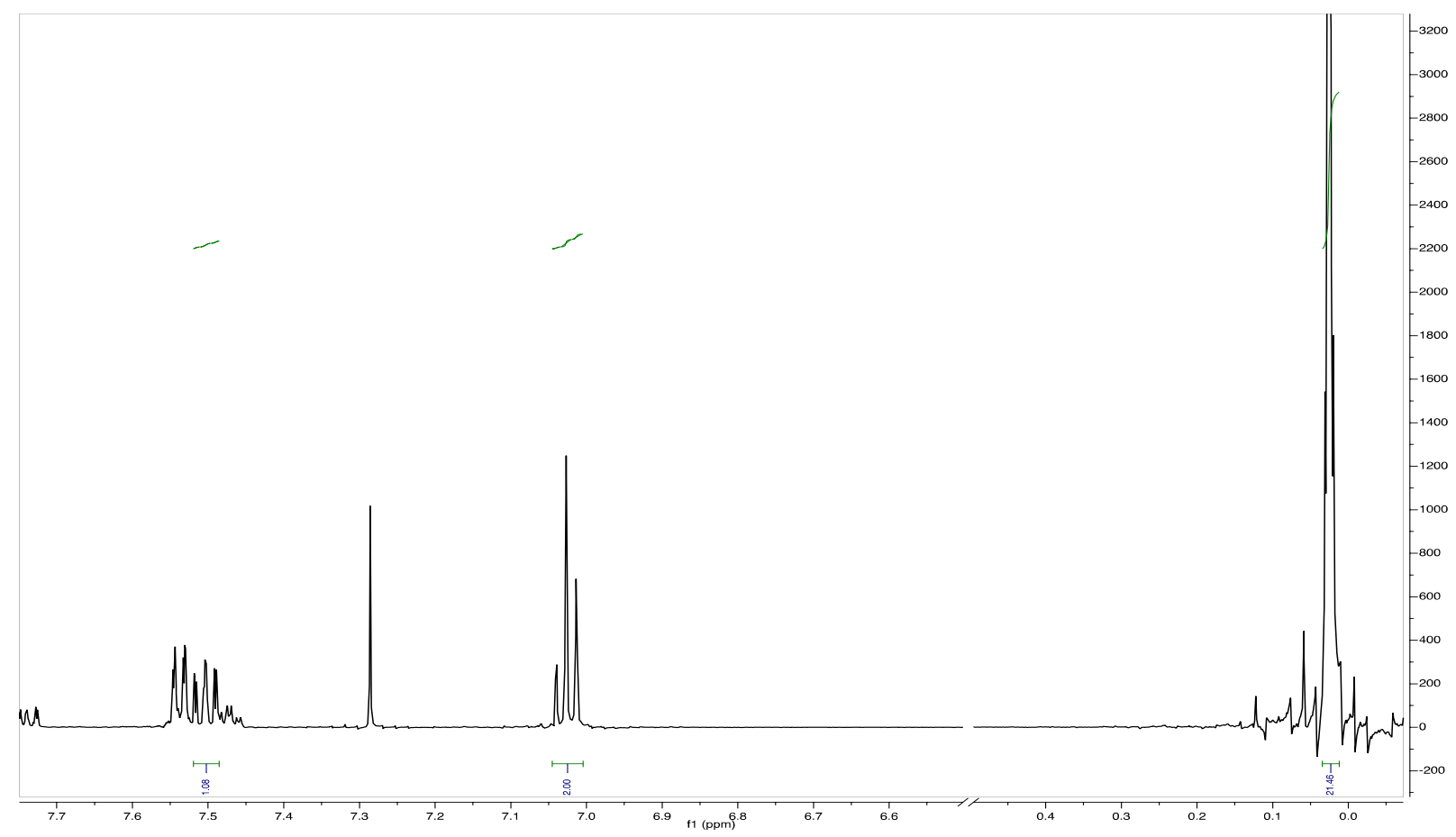

Example of ${ }^{1} \mathrm{H}\left(400 \mathrm{MHz}, \mathrm{CDCl}_{3}\right), 12 \mathrm{a}+$ catalyst 3 (triphenylphosphine sulfide); $\mathrm{t}=0 \mathrm{~h}$

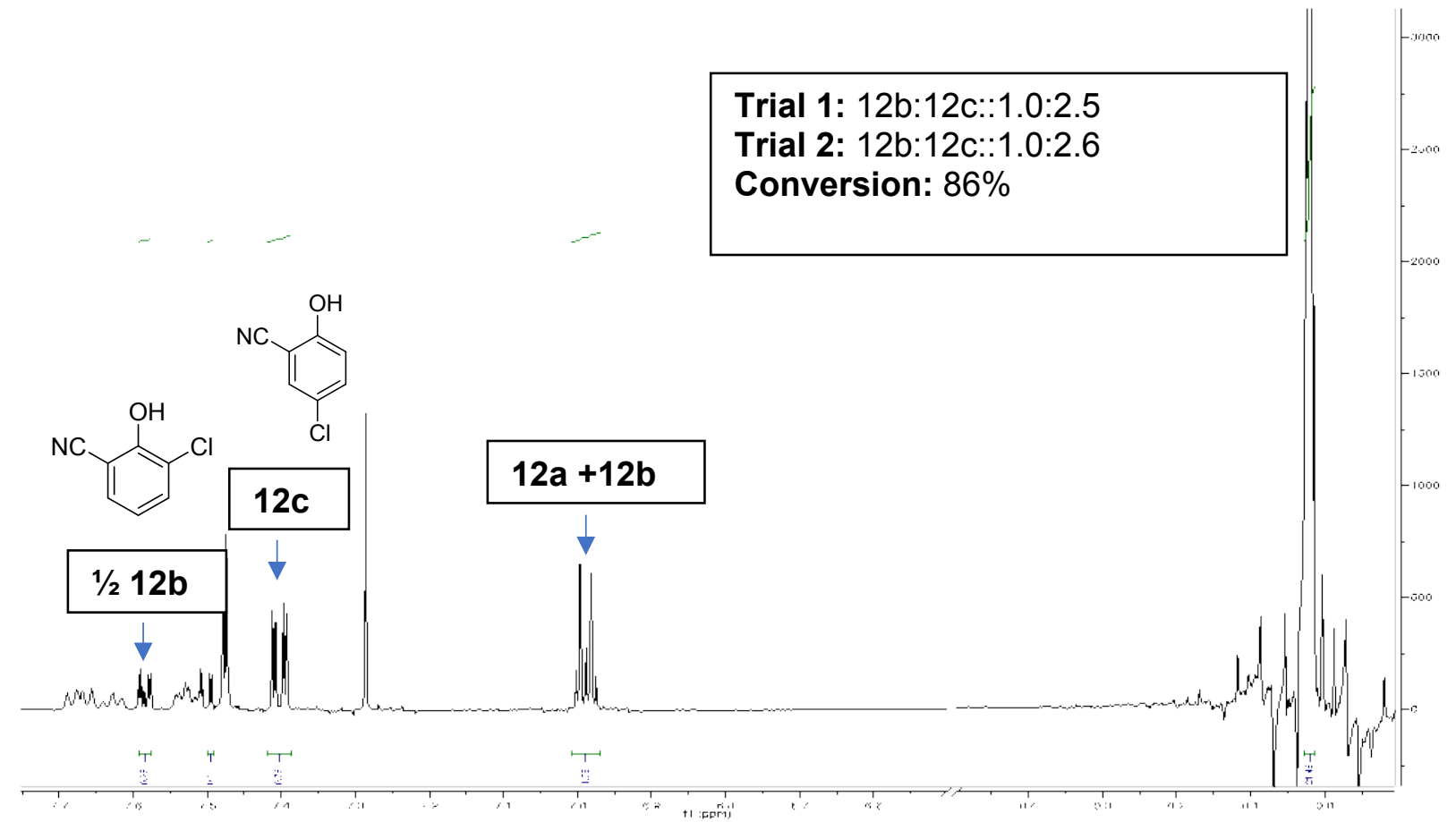

Example of ${ }^{1} \mathrm{H}\left(400 \mathrm{MHz}, \mathrm{CDCl}_{3}\right), 12 \mathrm{a}+$ catalyst 3 (triphenylphosphine sulfide). NMRs were taken from previously published literature in our laboratory and the results were reproduced. ${ }^{6}$ 


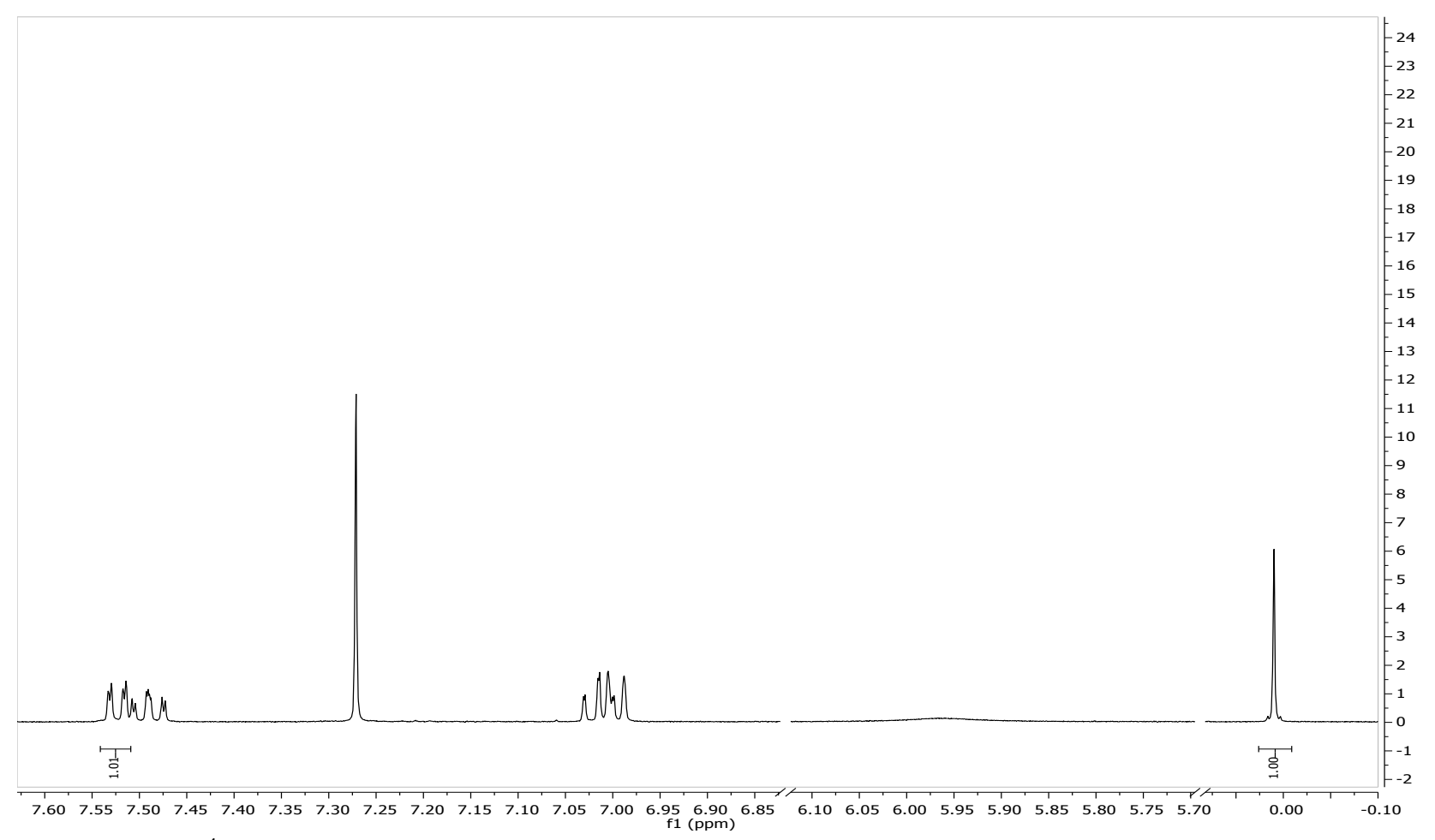

Example of ${ }^{1} \mathrm{H}\left(500 \mathrm{MHz}, \mathrm{CDCl}_{3}\right), 12 \mathrm{a}+$ catalyst $10 ; \mathrm{t}=0 \mathrm{~h}$

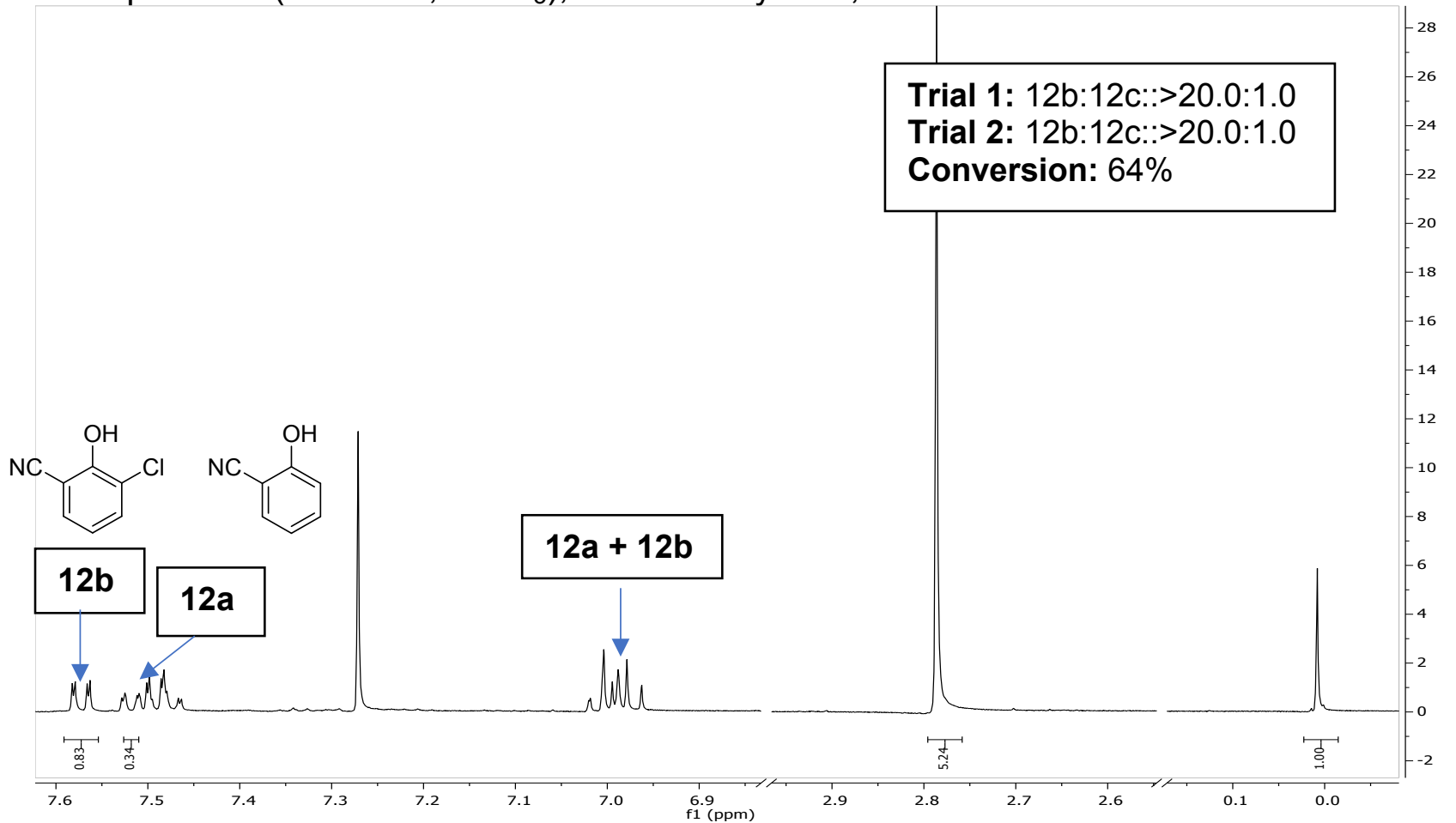

Example of ${ }^{1} \mathrm{H}\left(500 \mathrm{MHz}, \mathrm{CDCl}_{3}\right), 12 \mathrm{a}+$ catalyst $10 . t=12 \mathrm{~h} .12 \mathrm{c}$ is not observed in crude NMR. 


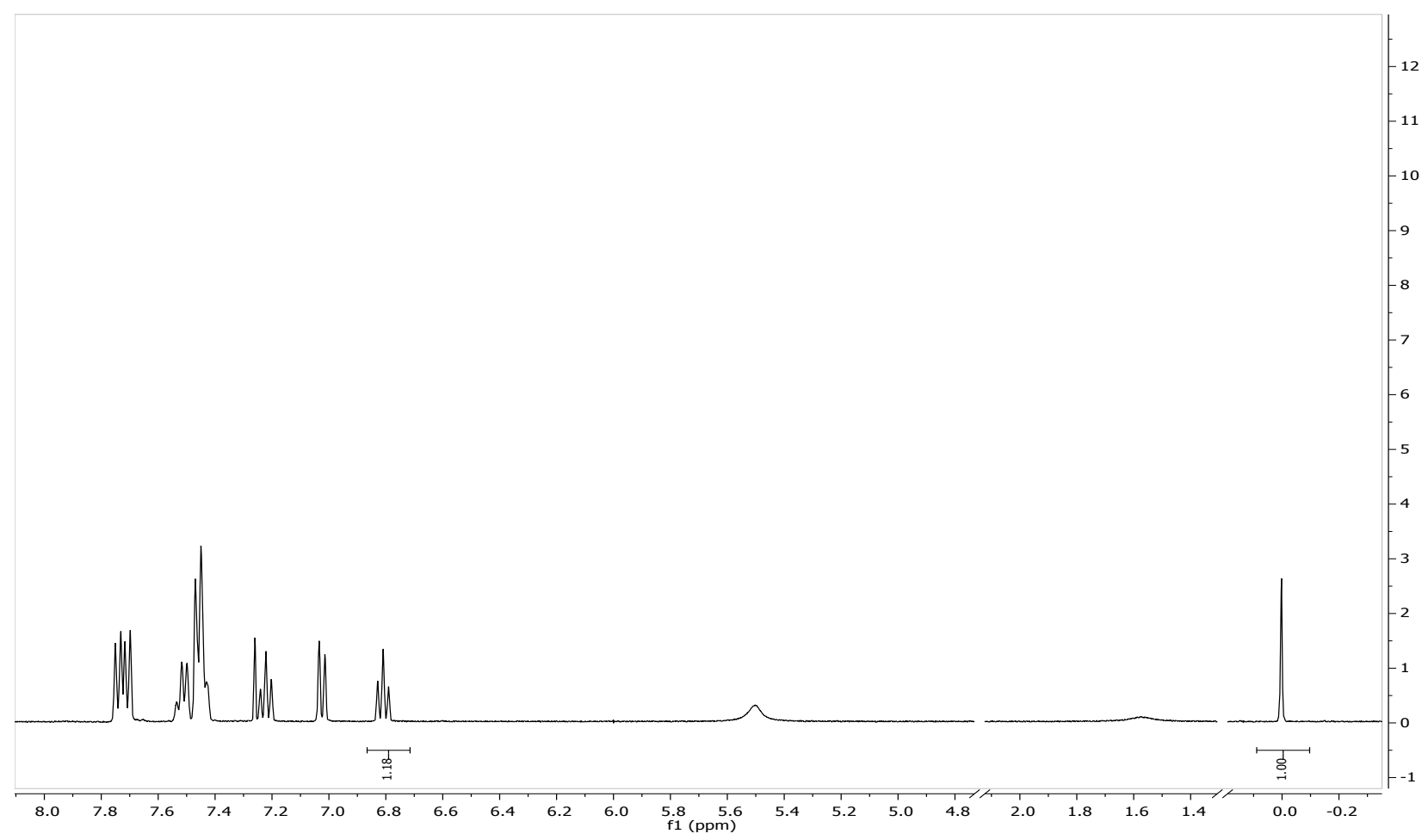

Example of ${ }^{1} \mathrm{H}\left(500 \mathrm{MHz}, \mathrm{CDCl}_{3}\right), 13 \mathrm{a}+$ catalyst 3 (triphenylphosphine sulfide); $\mathrm{t}=0 \mathrm{~h}$

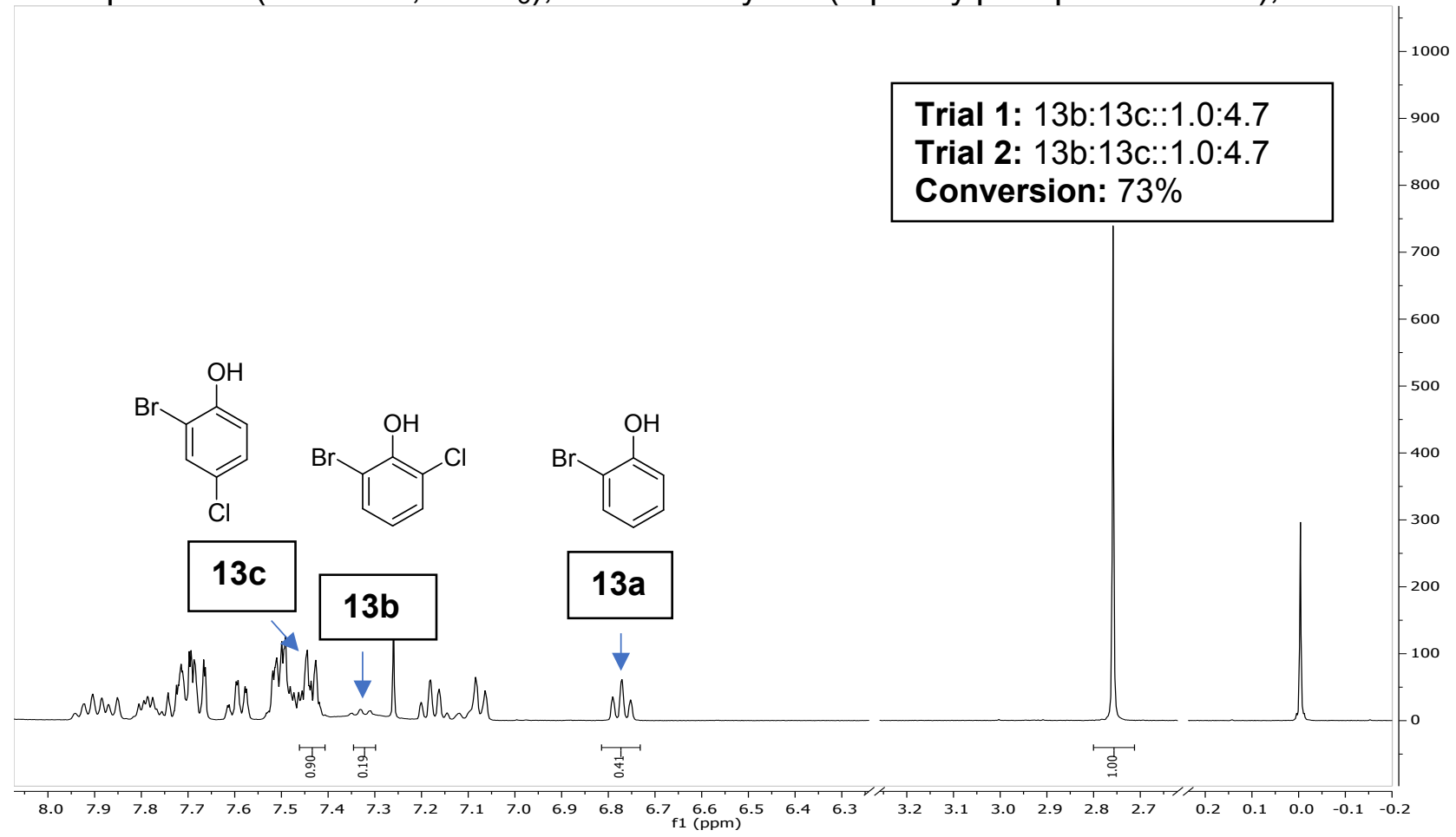

Example of ${ }^{1} \mathrm{H}\left(400 \mathrm{MHz}, \mathrm{CDCl}_{3}\right), 13 \mathrm{a}+$ catalyst 3 (triphenylphosphine sulfide). $\mathrm{t}=12 \mathrm{~h}$. 


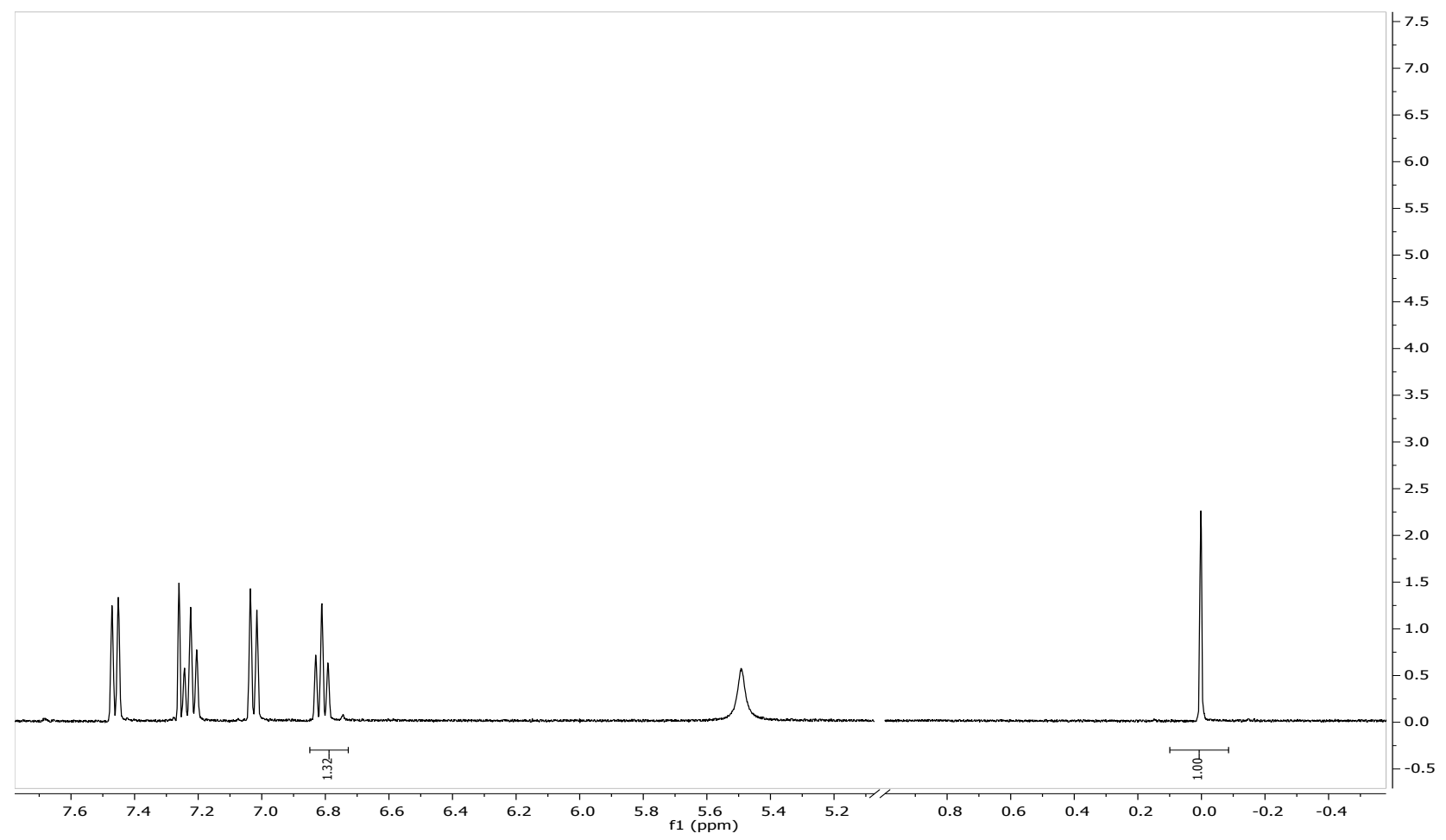

Example of ${ }^{1} \mathrm{H}\left(500 \mathrm{MHz}, \mathrm{CDCl}_{3}\right), 13 \mathrm{a}+$ catalyst $10 ; \mathrm{t}=0 \mathrm{~h}$

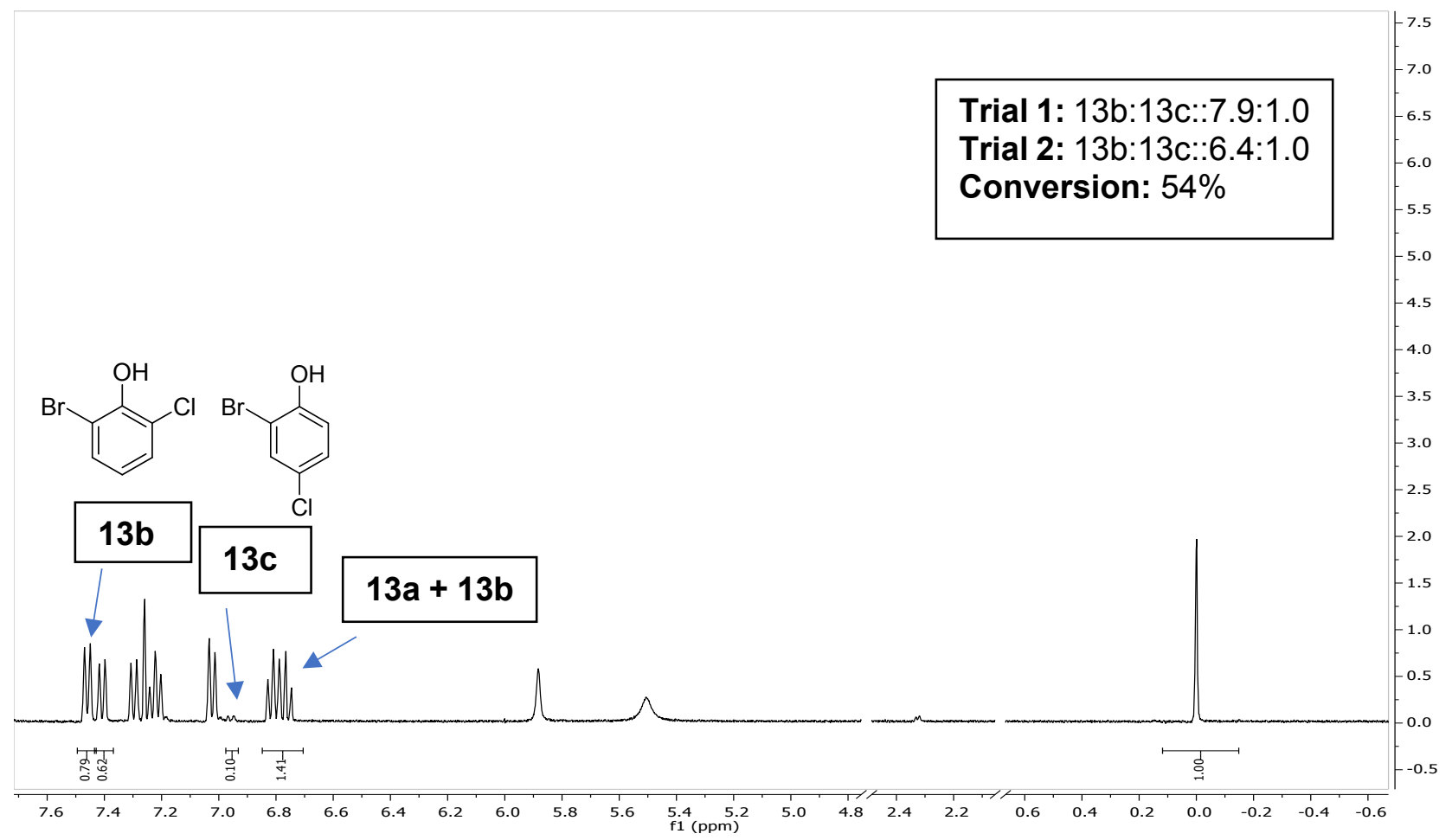

Example of ${ }^{1} \mathrm{H}\left(500 \mathrm{MHz}, \mathrm{CDCl}_{3}\right), 13 \mathrm{a}+$ catalyst $10 ; \mathrm{t}=12 \mathrm{~h}$. 


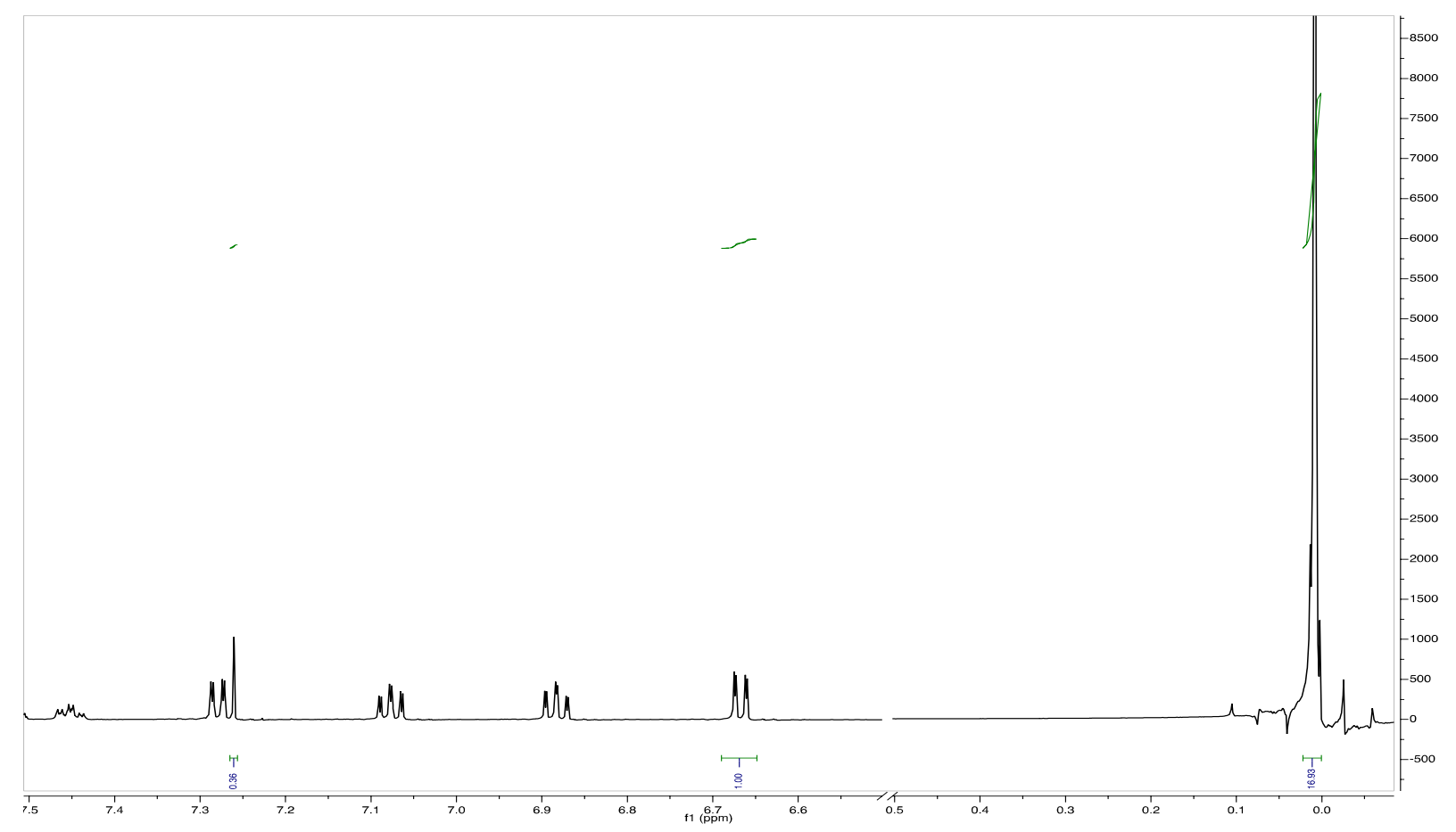

Example of ${ }^{1} \mathrm{H}\left(400 \mathrm{MHz}, \mathrm{CDCl}_{3}\right), 14 \mathrm{a}+$ catalyst 3 (triphenylphosphine sulfide); $\mathrm{t}=0 \mathrm{~h}$

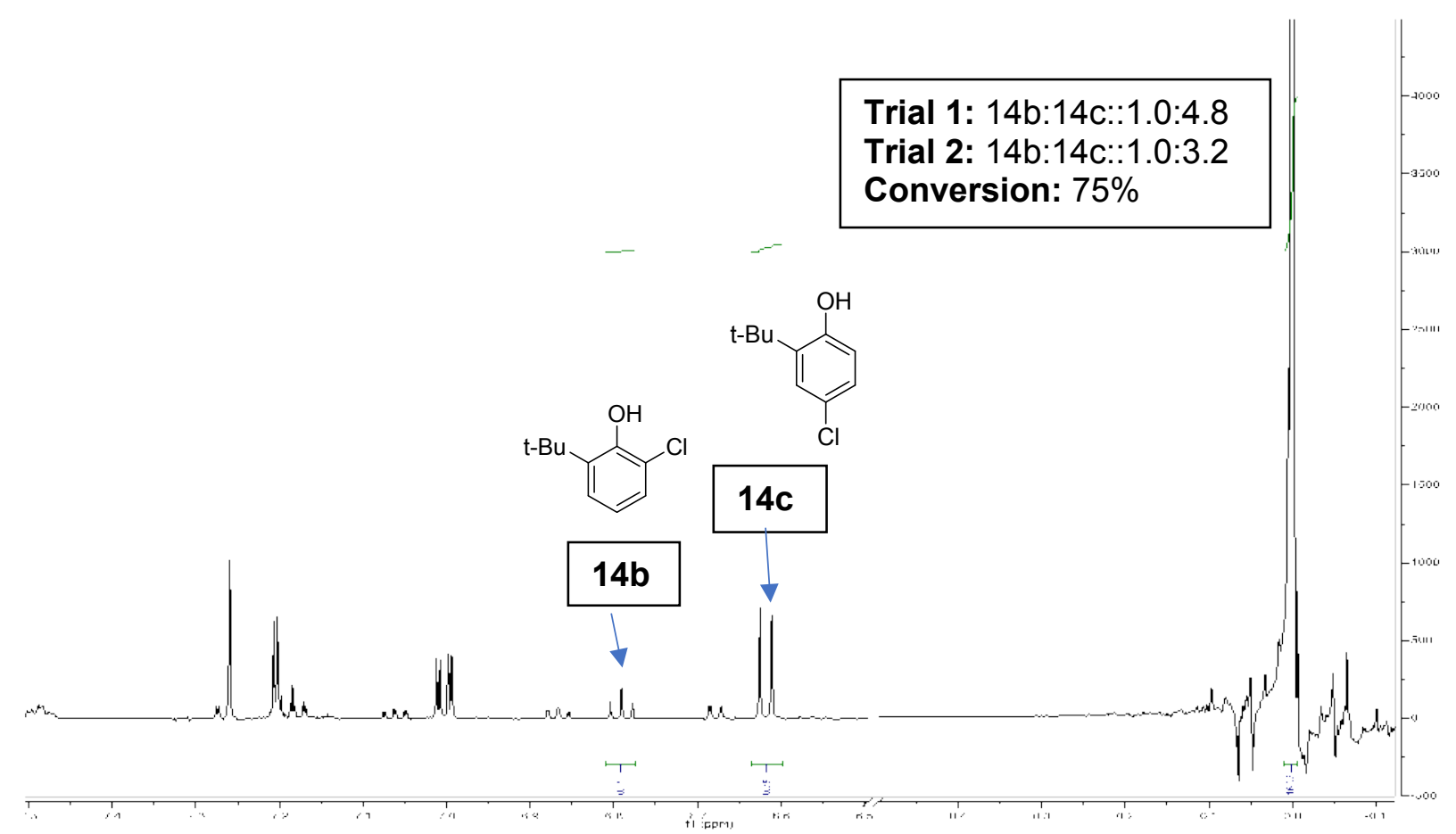

Example of ${ }^{1} \mathrm{H}\left(400 \mathrm{MHz}, \mathrm{CDCl}_{3}\right), 14 \mathrm{a}+$ catalyst 3 (triphenylphosphine sulfide); $\mathrm{t}=12 \mathrm{~h}$. NMRs were taken from previously published literature in our laboratory and the results were reproduced. ${ }^{6}$ 


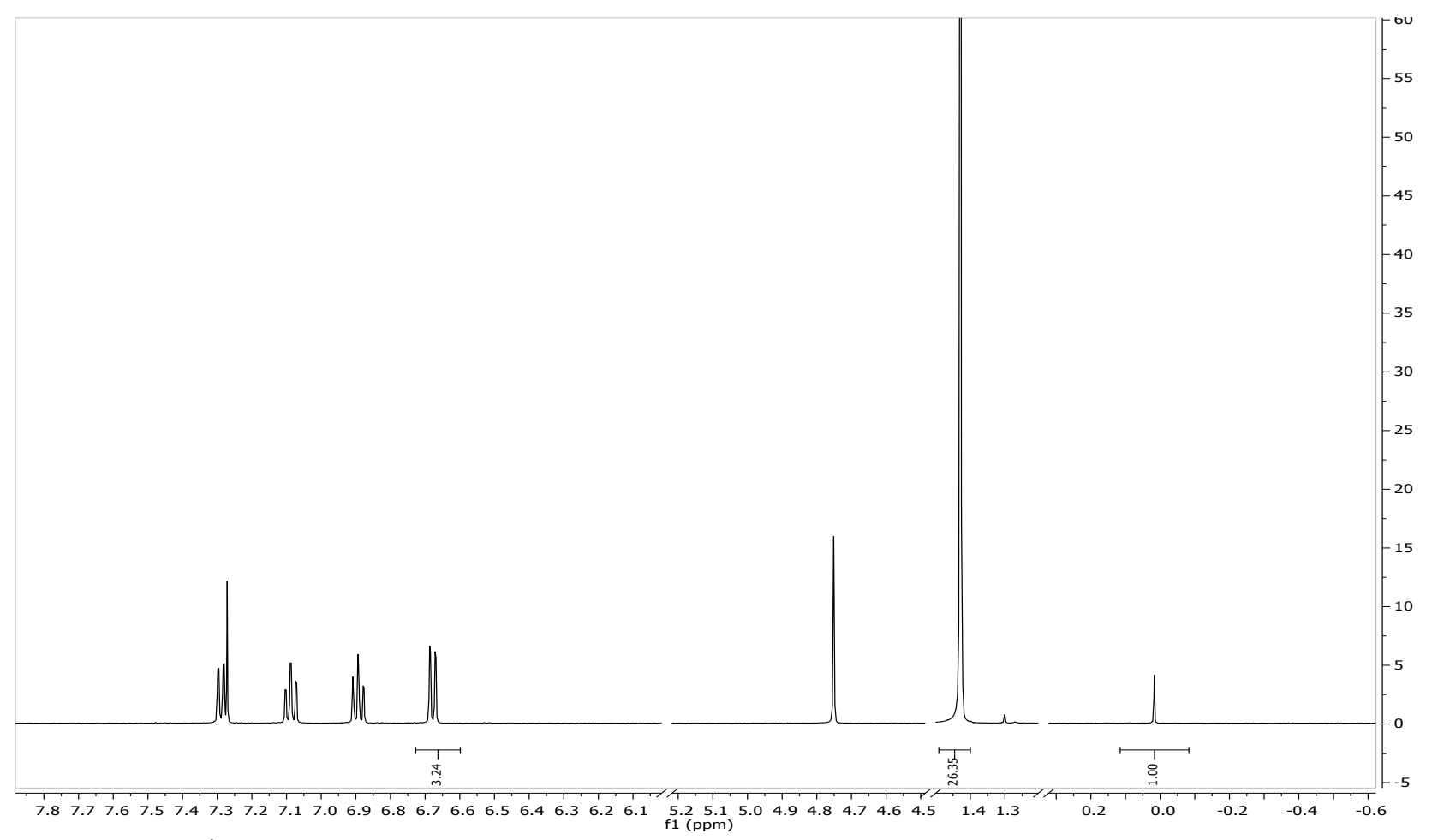

Example of ${ }^{1} \mathrm{H}\left(500 \mathrm{MHz}, \mathrm{CDCl}_{3}\right), 14 \mathrm{a}+$ catalyst $10 ; \mathrm{t}=0 \mathrm{~h}$

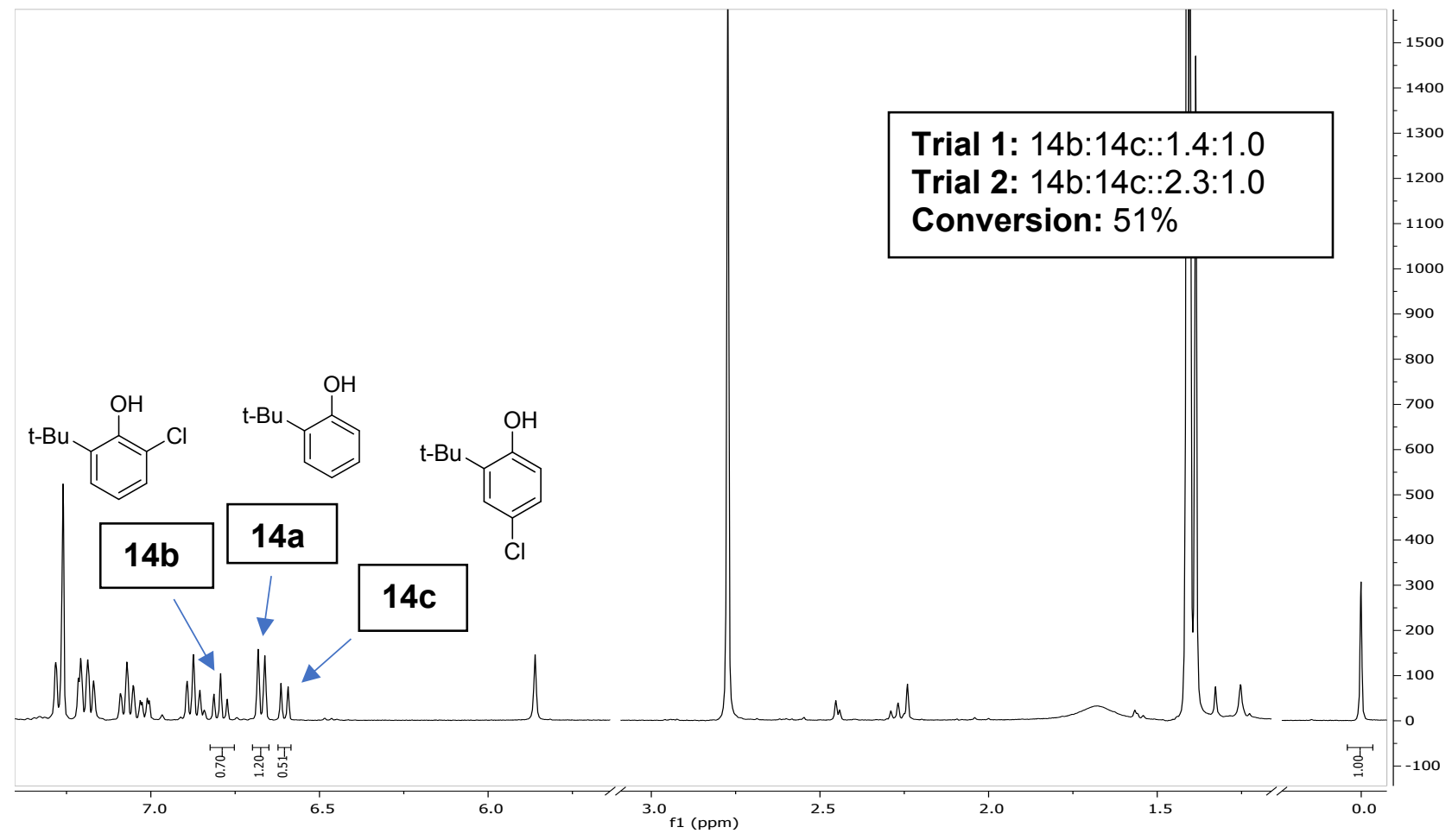

Example of ${ }^{1} \mathrm{H}\left(400 \mathrm{MHz}, \mathrm{CDCl}_{3}\right), 14 \mathrm{a}+$ catalyst $10 ; \mathrm{t}=12 \mathrm{~h}$. 


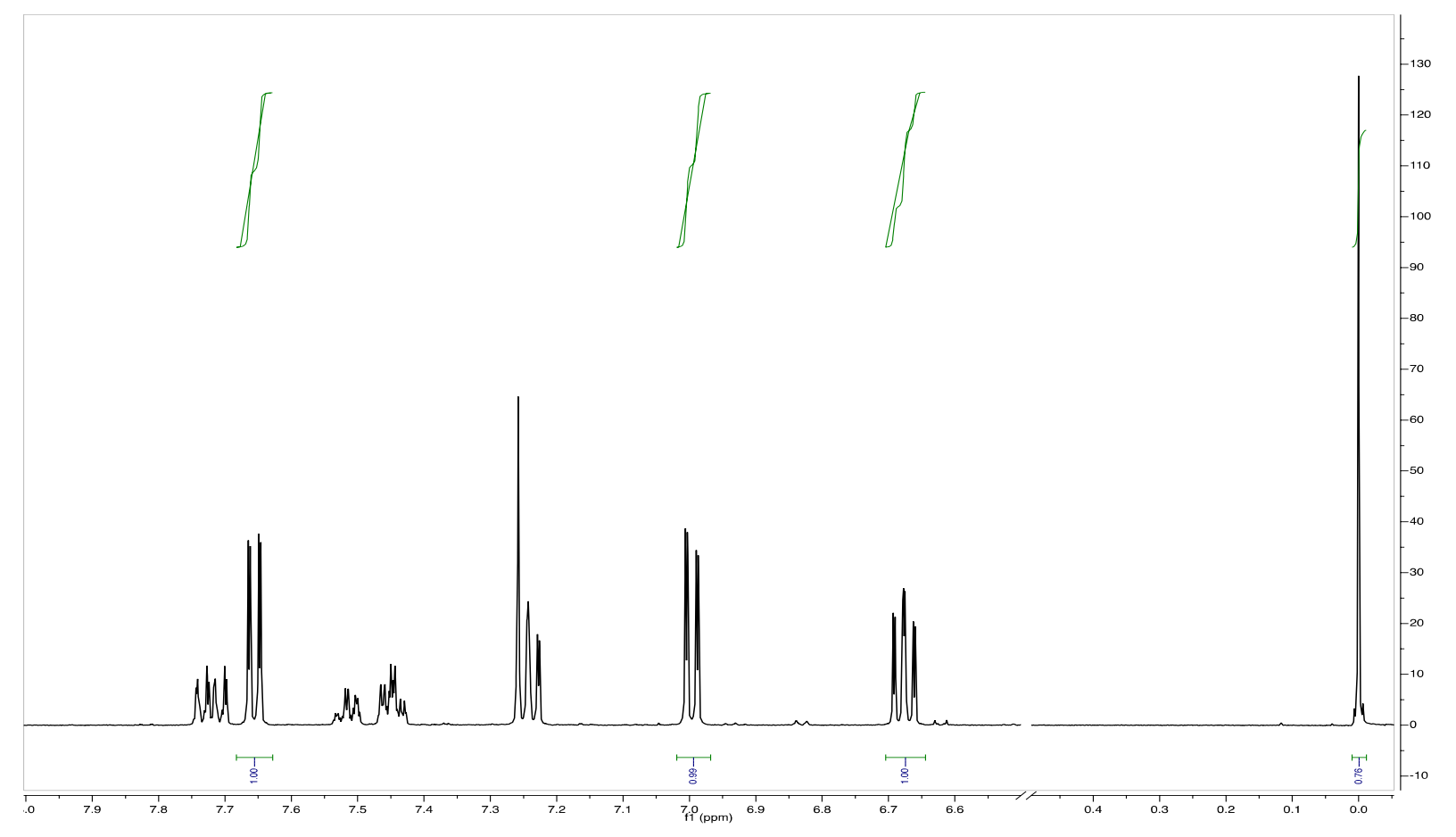

Example of ${ }^{1} \mathrm{H}\left(400 \mathrm{MHz}, \mathrm{CDCl}_{3}\right), 15 \mathrm{a}+$ catalyst 3 (triphenylphosphine sulfide); $\mathrm{t}=0 \mathrm{~h}$

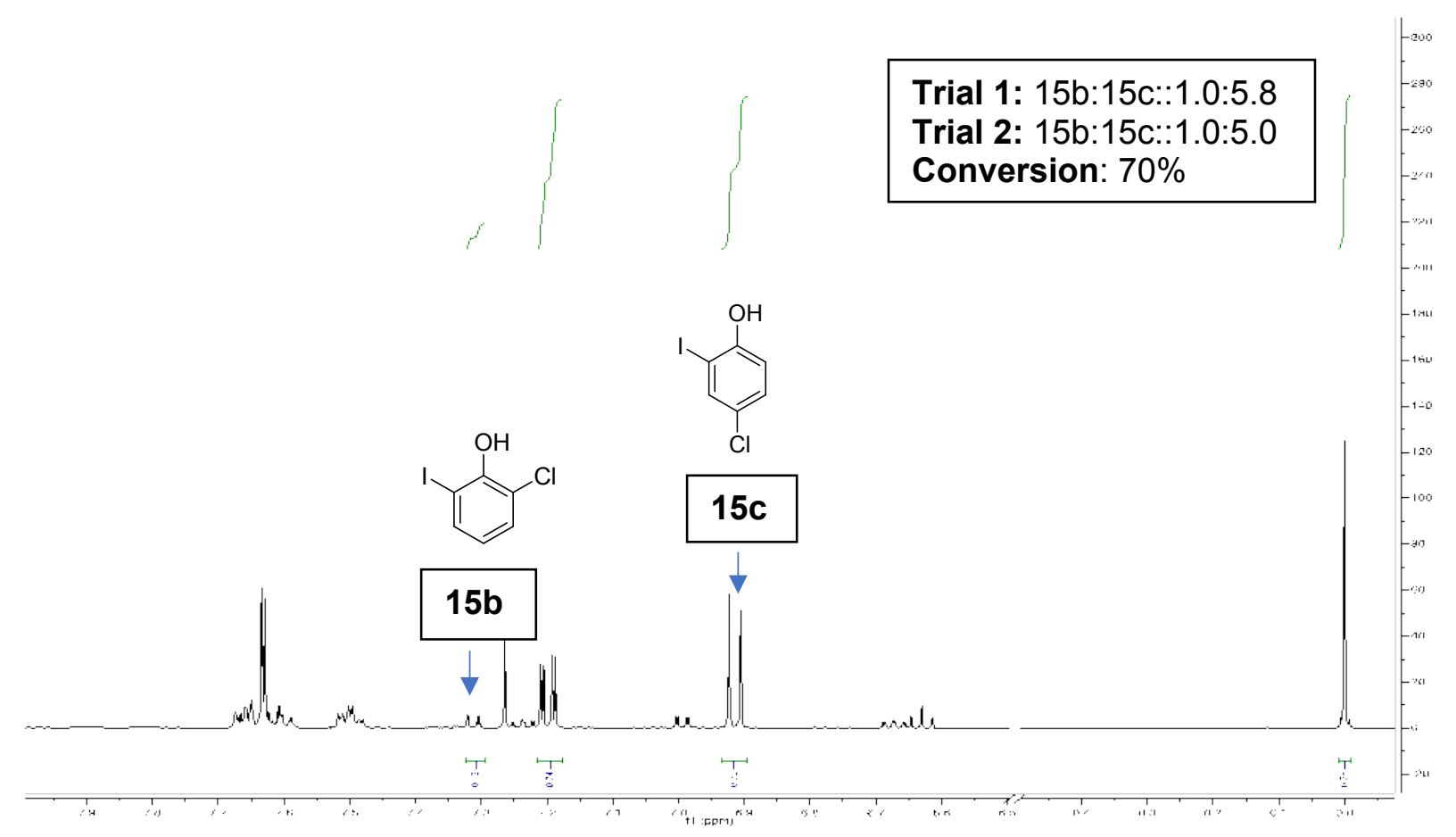

Example of ${ }^{1} \mathrm{H}\left(400 \mathrm{MHz}, \mathrm{CDCl}_{3}\right), 15 \mathrm{a}+$ catalyst 3 (triphenylphosphine sulfide); $\mathrm{t}=12 \mathrm{~h}$. NMRs were taken from previously published literature in our laboratory and the results were reproduced. ${ }^{6}$ 


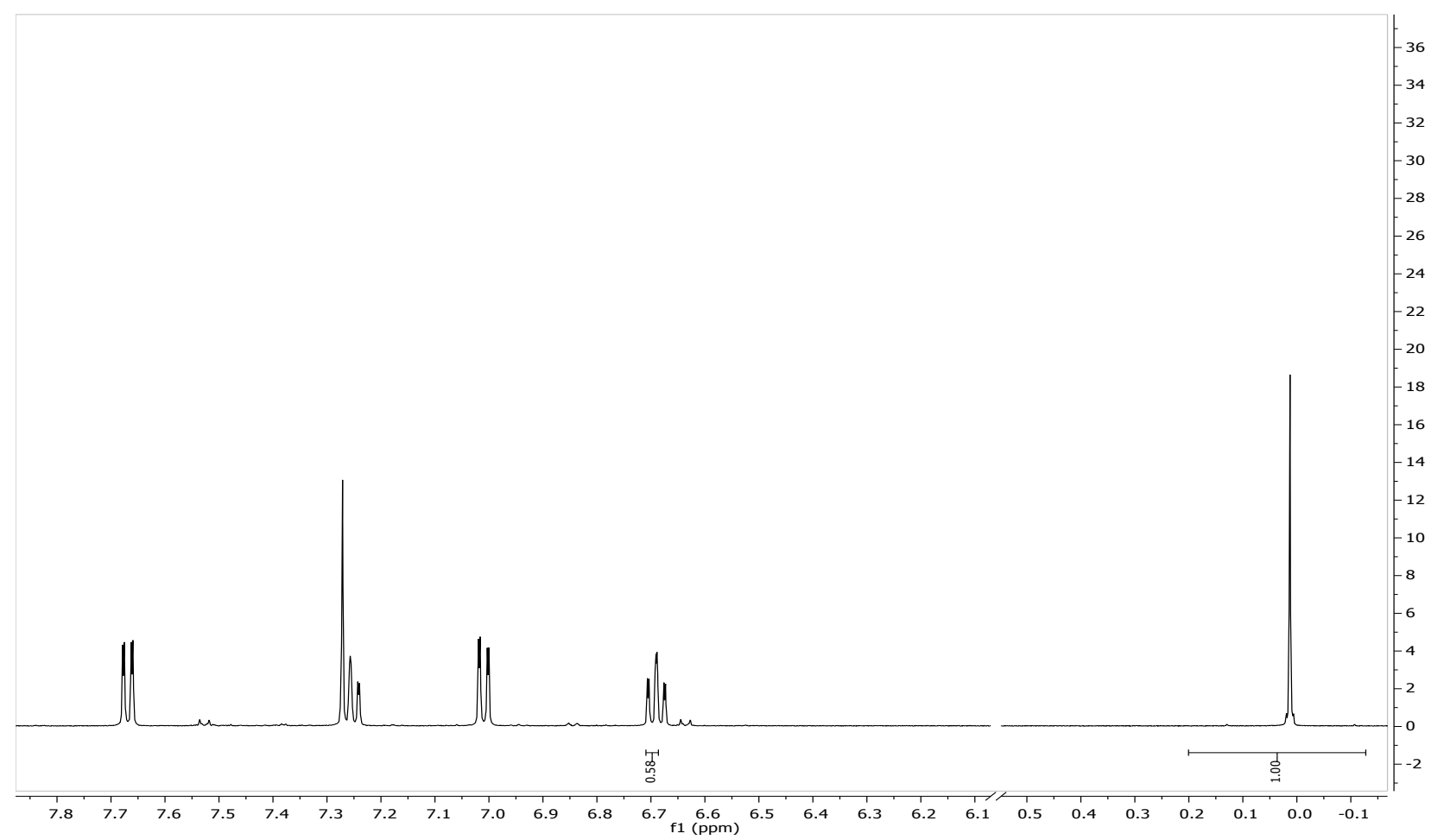

Example of ${ }^{1} \mathrm{H}\left(500 \mathrm{MHz}, \mathrm{CDCl}_{3}\right), 15 \mathrm{a}+$ catalyst $10 ; \mathrm{t}=0 \mathrm{~h}$

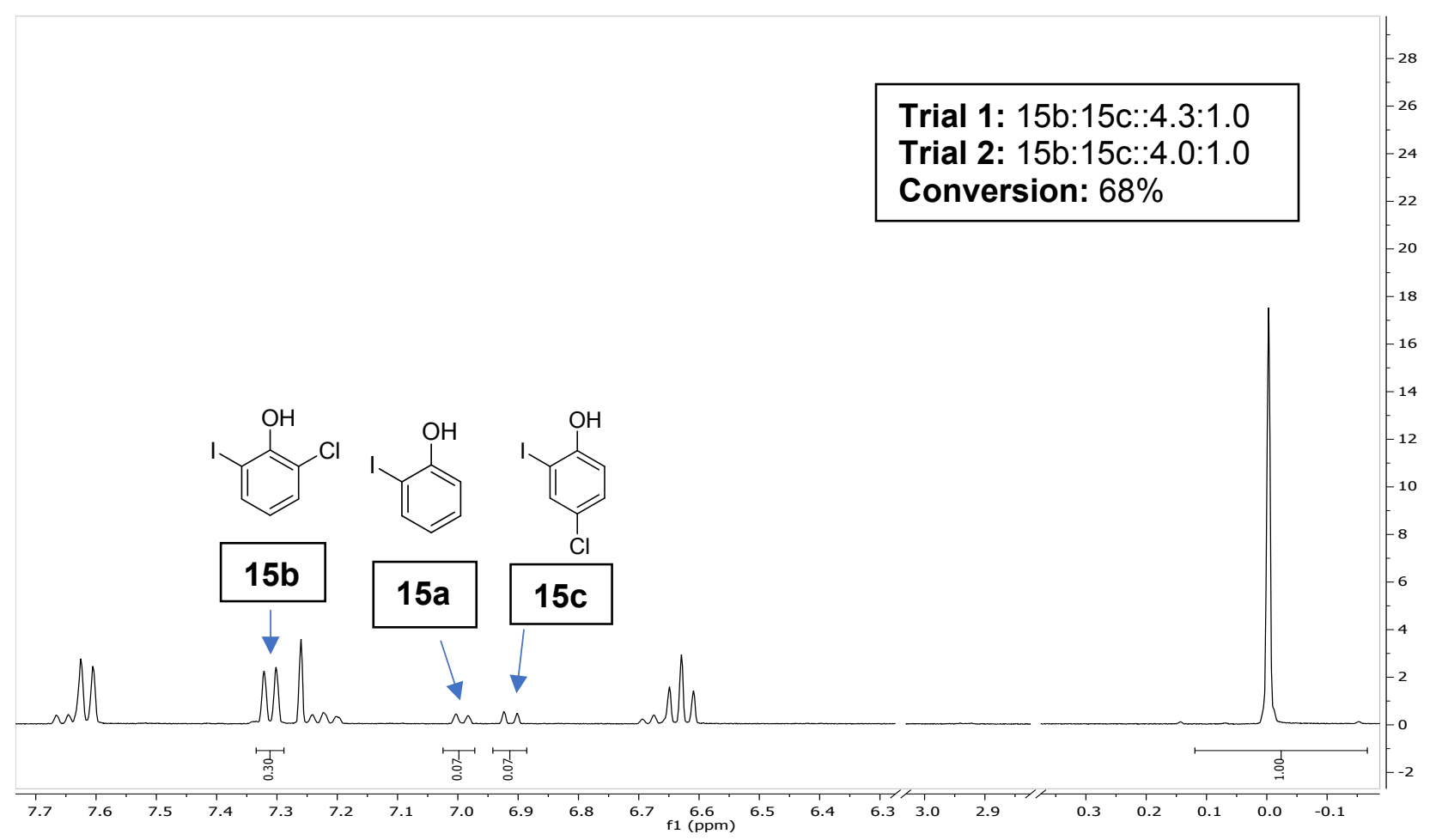

Example of ${ }^{1} \mathrm{H}\left(500 \mathrm{MHz}, \mathrm{CDCl}_{3}\right), 15 \mathrm{a}+$ catalyst $10 ; \mathrm{t}=12 \mathrm{~h}$ 


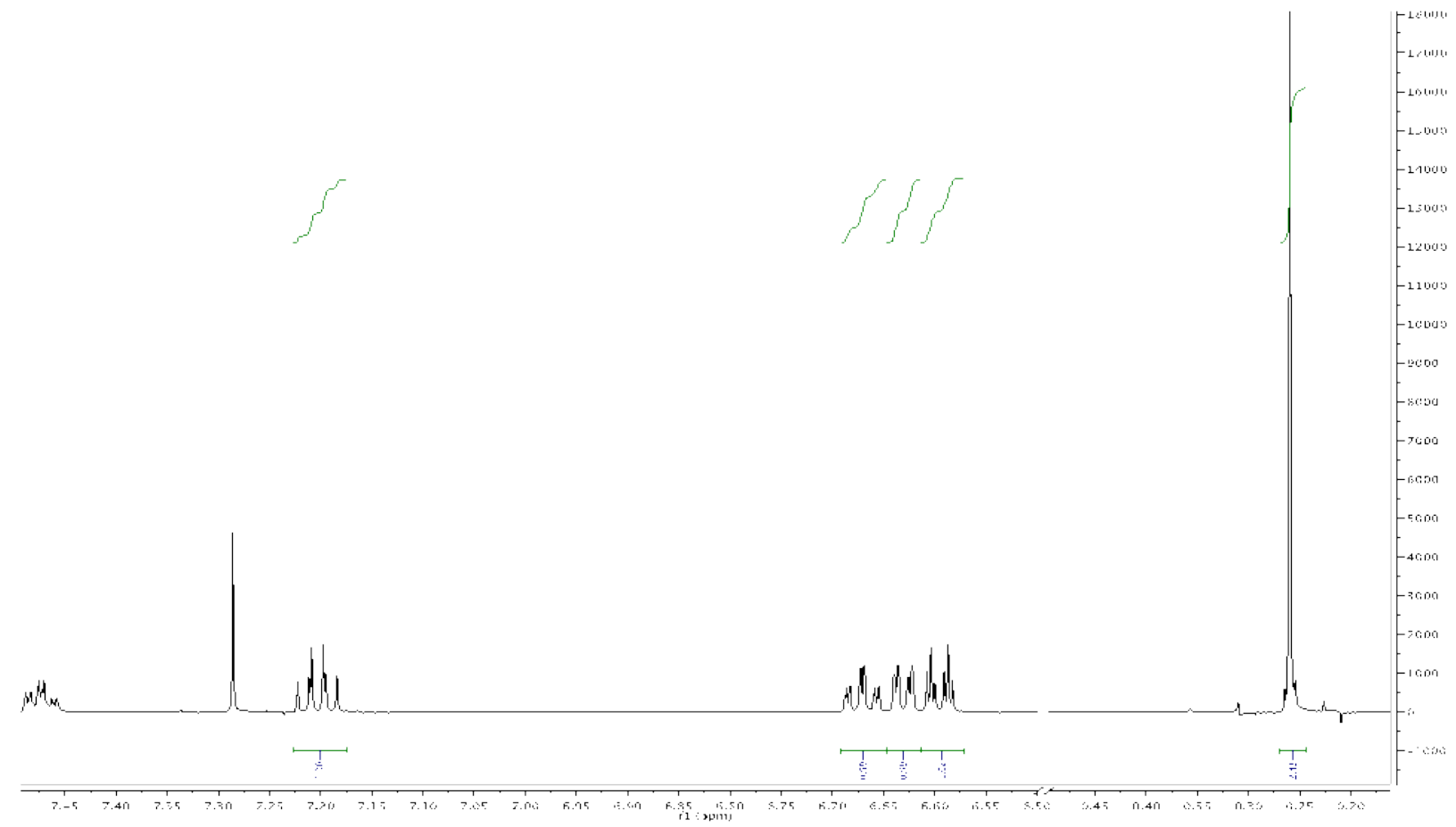

Example of ${ }^{1} \mathrm{H}\left(400 \mathrm{MHz}, \mathrm{CDCl}_{3}\right), 16 \mathrm{a}+$ catalyst 3 (triphenylphosphine sulfide); $\mathrm{t}=0 \mathrm{~h}$

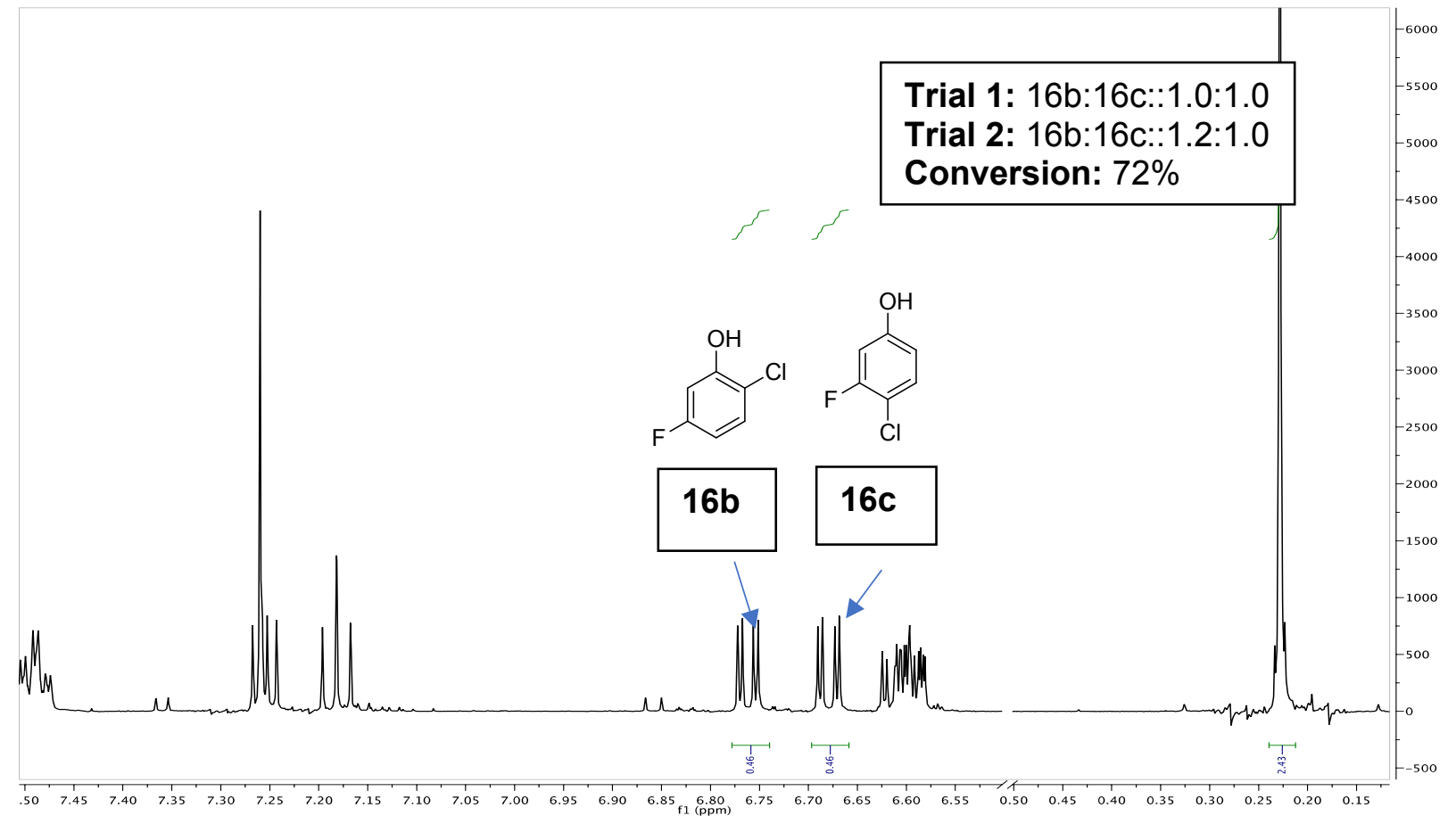

Example of ${ }^{1} \mathrm{H} 400 \mathrm{MHz}, \mathrm{CDCl}_{3}$ ), $16 \mathrm{a}+$ catalyst 3 (triphenylphosphine sulfide); $\mathrm{t}=12 \mathrm{~h}$. NMRs were taken from previously published literature in our laboratory and the results were reproduced. ${ }^{6}$ 


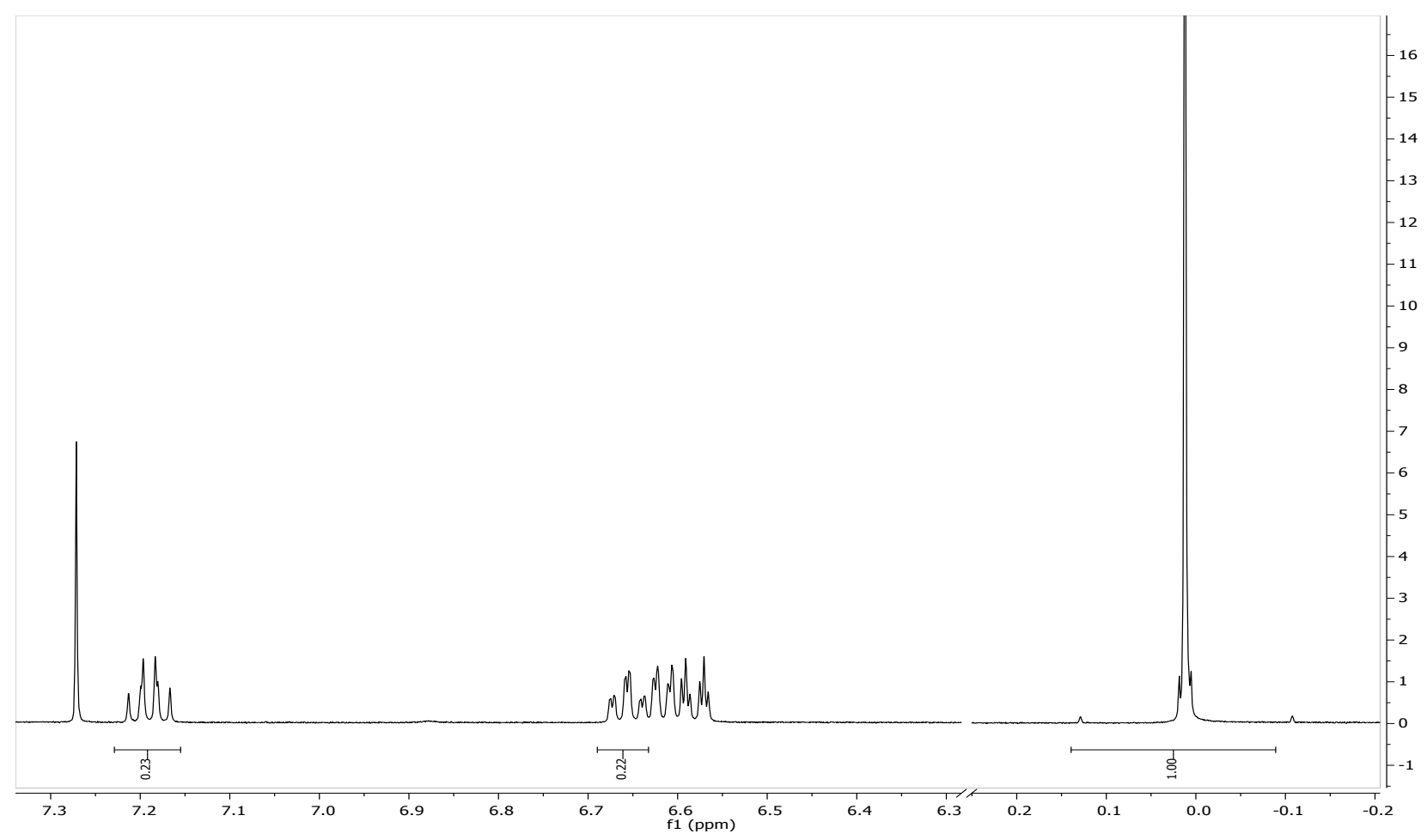

Example of ${ }^{1} \mathrm{H}\left(500 \mathrm{MHz}, \mathrm{CDCl}_{3}\right), 16 \mathrm{a}+$ catalyst $10 ; \mathrm{t}=0 \mathrm{~h}$

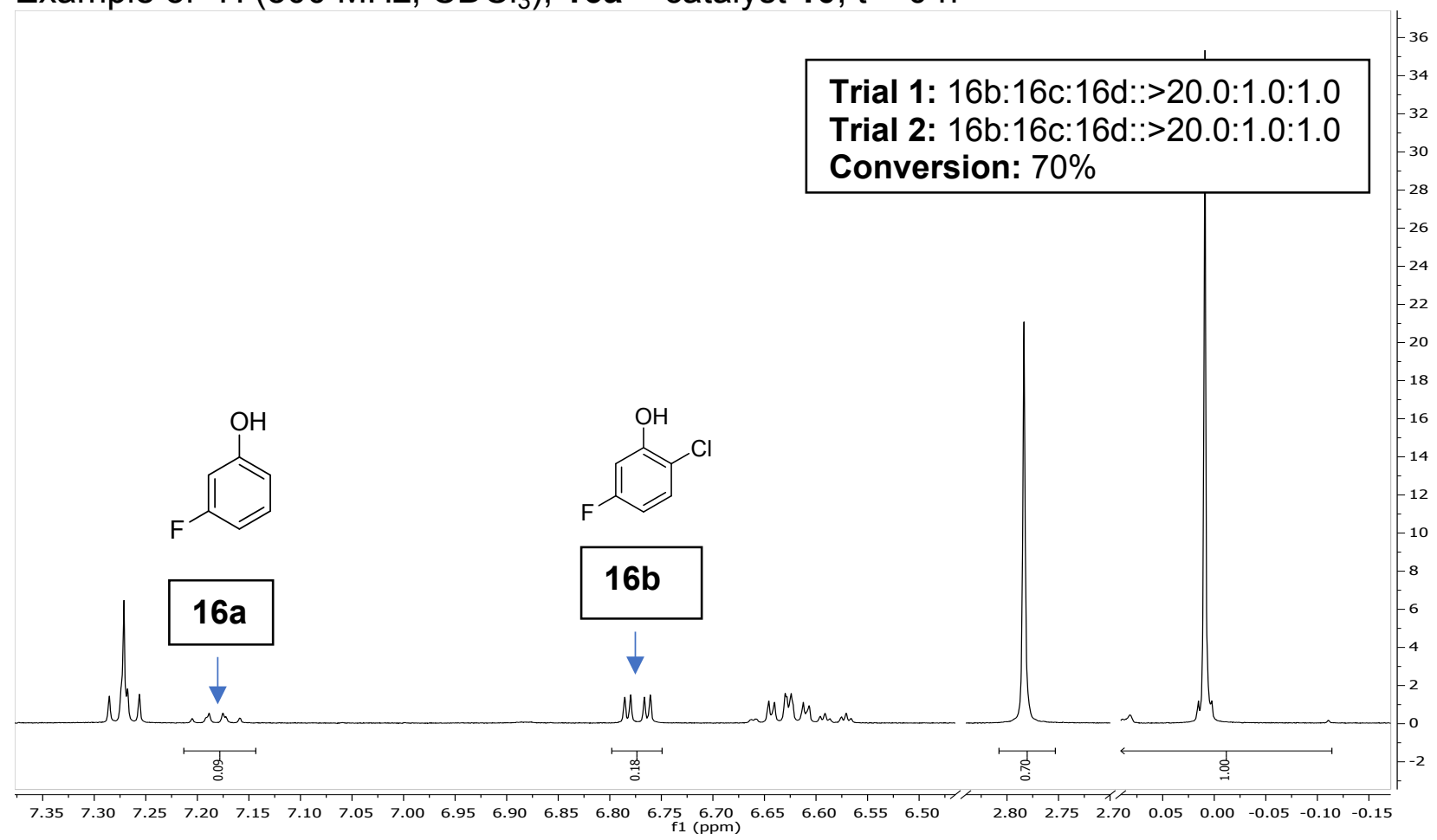

Example of ${ }^{1} \mathrm{H}\left(500 \mathrm{MHz}, \mathrm{CDCl}_{3}\right), 16 \mathbf{a}+$ catalyst $10 ; \mathrm{t}=12 \mathrm{~h}$. No presence of $16 \mathrm{c}$ and $16 \mathrm{~d}$ was observed but the ratio was used to calculate a quantitative delta $\mathrm{G}$ value. 


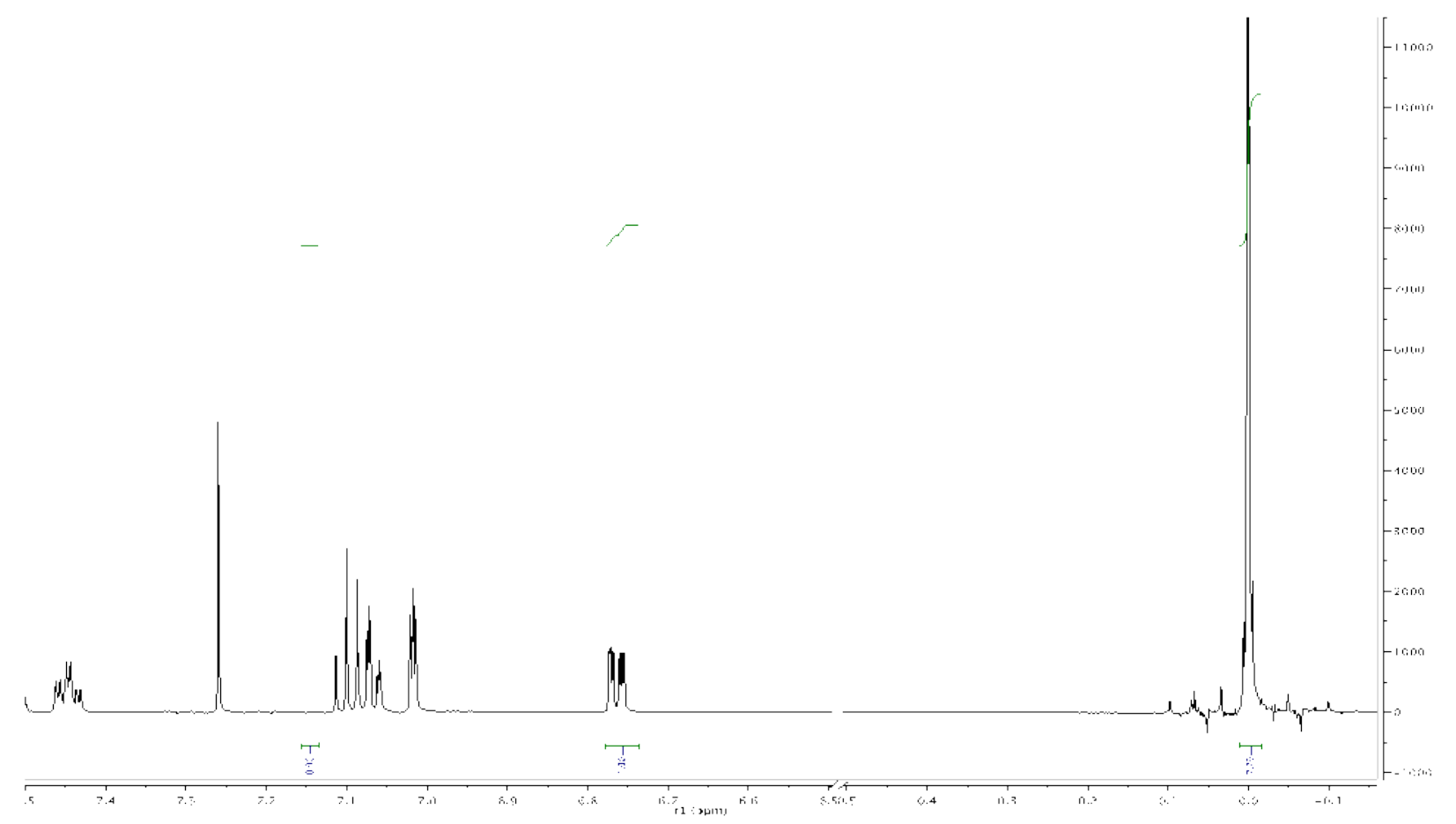

Example of ${ }^{1} \mathrm{H}\left(400 \mathrm{MHz}, \mathrm{CDCl}_{3}\right), 17 \mathrm{a}+$ catalyst 3 (triphenylphosphine sulfide); $\mathrm{t}=0 \mathrm{~h}$

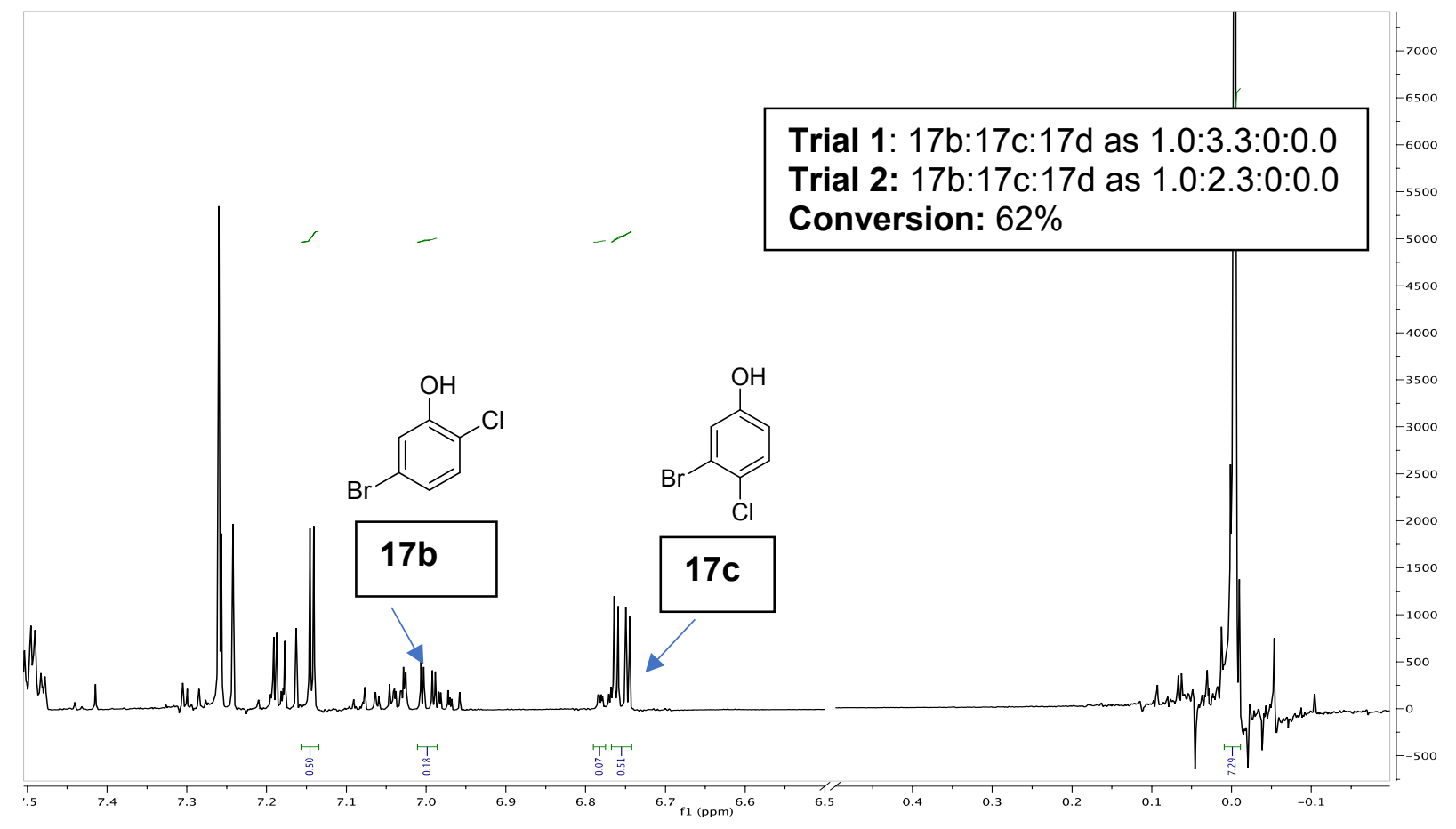

Example of ${ }^{1} \mathrm{H}\left(400 \mathrm{MHz}, \mathrm{CDCl}_{3}\right), 17 \mathrm{a}+$ catalyst 3 (triphenylphosphine sulfide); $\mathrm{t}=3 \mathrm{~h}$. NMRs were taken from previously published literature in our laboratory and the results were reproduced. ${ }^{6}$ 


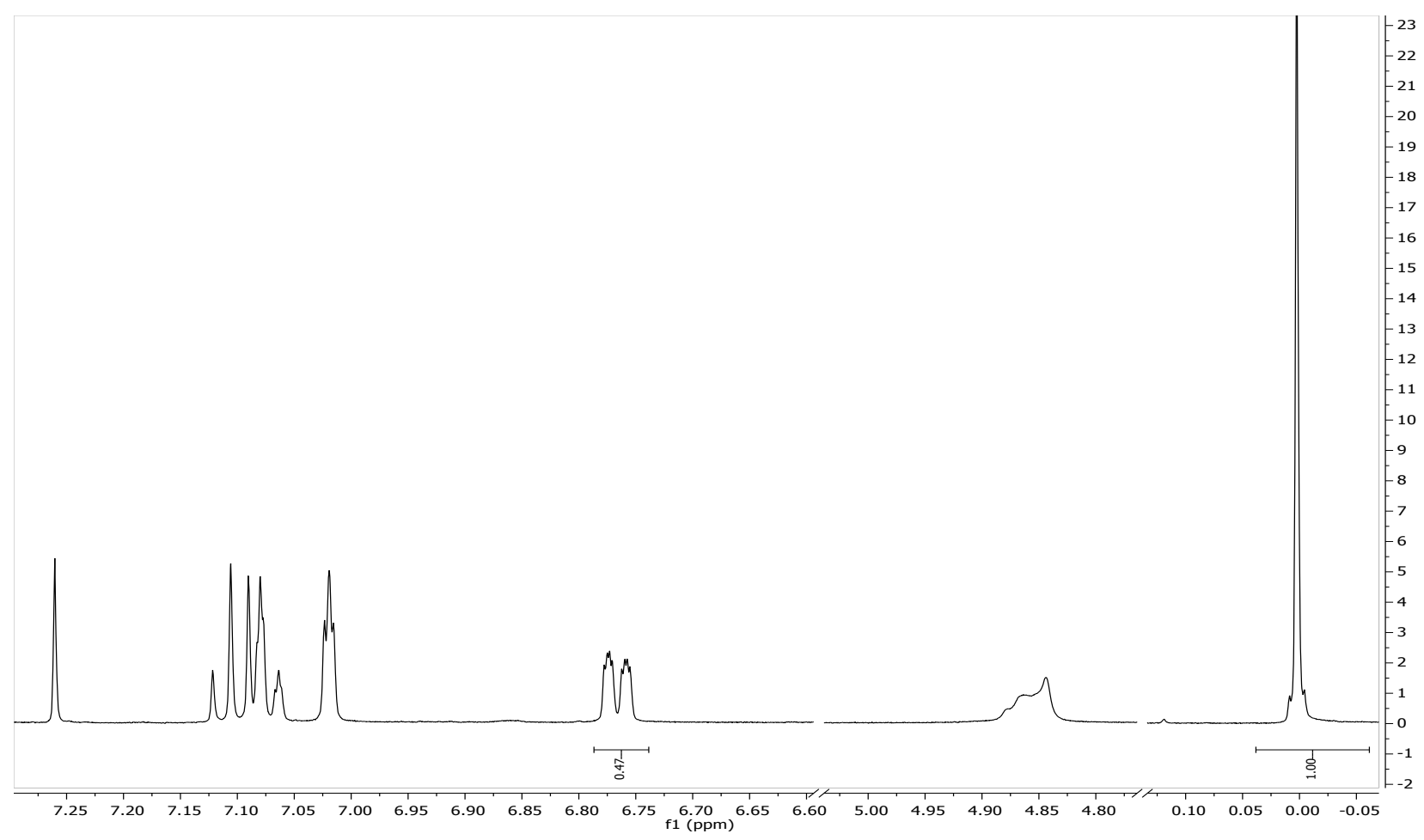

Example of ${ }^{1} \mathrm{H}\left(500 \mathrm{MHz}, \mathrm{CDCl}_{3}\right), 17 \mathrm{a}+$ catalyst $10 ; \mathrm{t}=0 \mathrm{~h}$

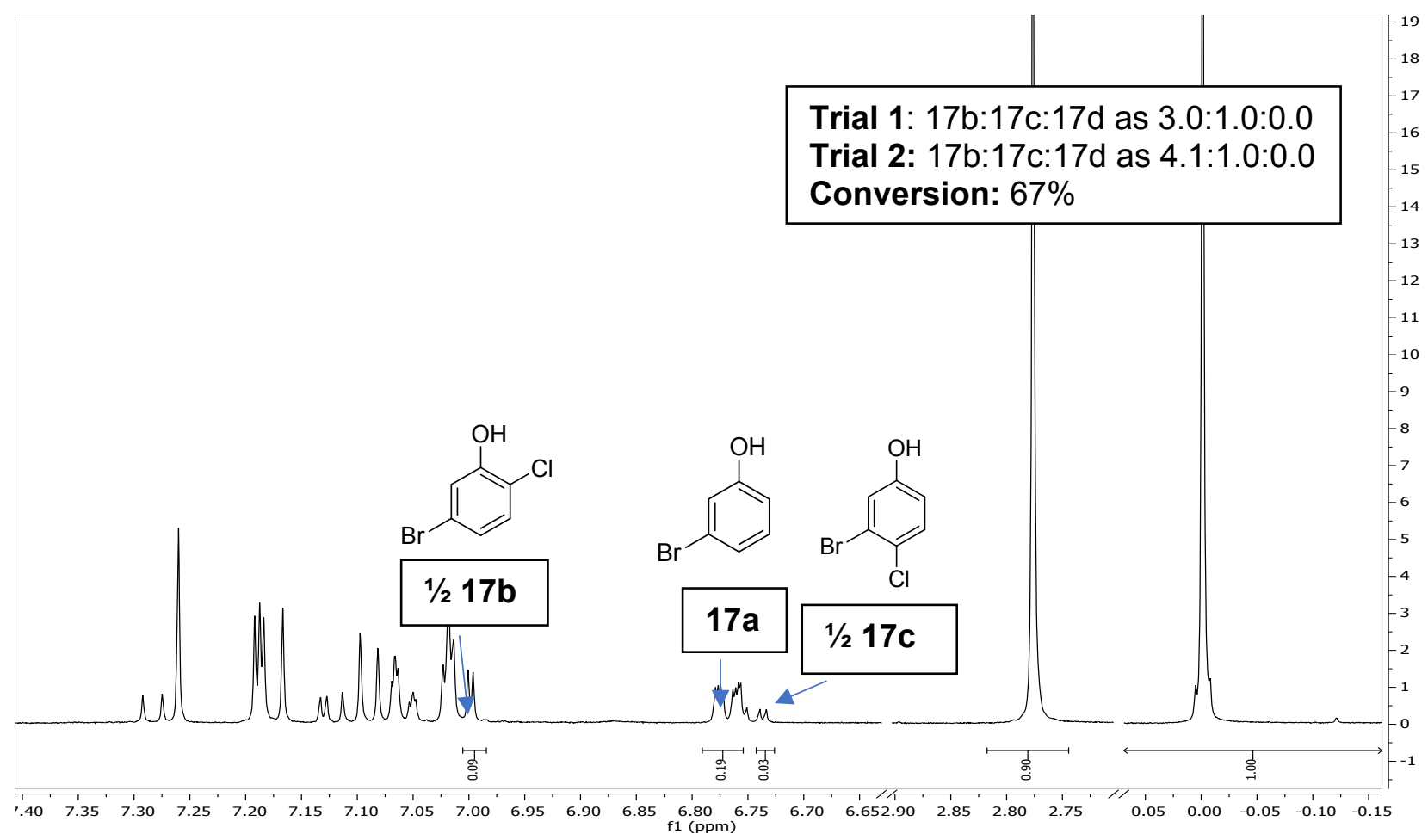

Example of ${ }^{1} \mathrm{H}\left(500 \mathrm{MHz}, \mathrm{CDCl}_{3}\right), 17 \mathrm{a}+$ catalyst $10 ; \mathrm{t}=12 \mathrm{~h}$ 


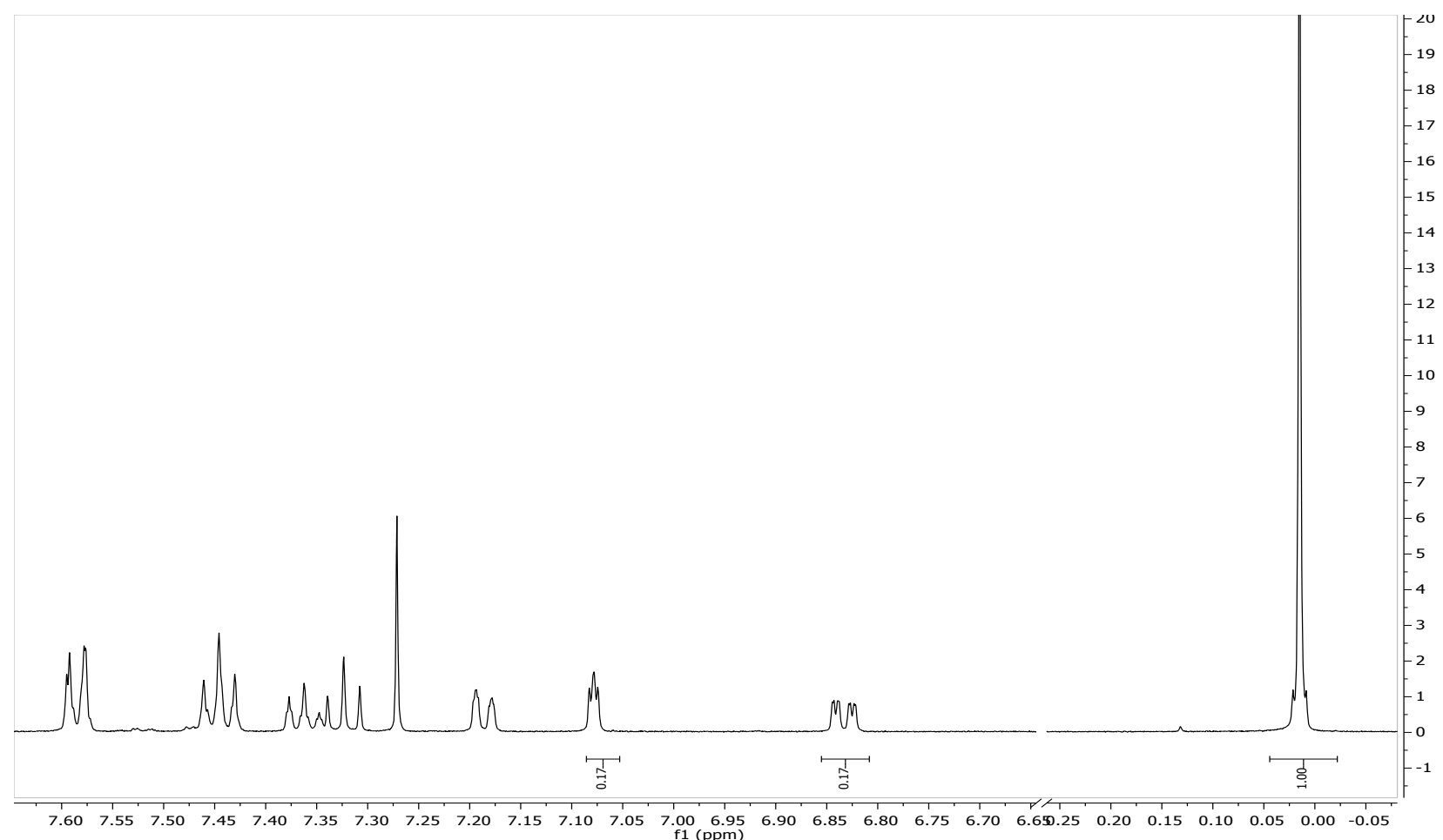

Example of ${ }^{1} \mathrm{H}\left(500 \mathrm{MHz}, \mathrm{CDCl}_{3}\right), \mathbf{1 8 a}+$ catalyst $\mathbf{3}$ (triphenylphosphine sulfide); $\mathrm{t}=0 \mathrm{~h}$

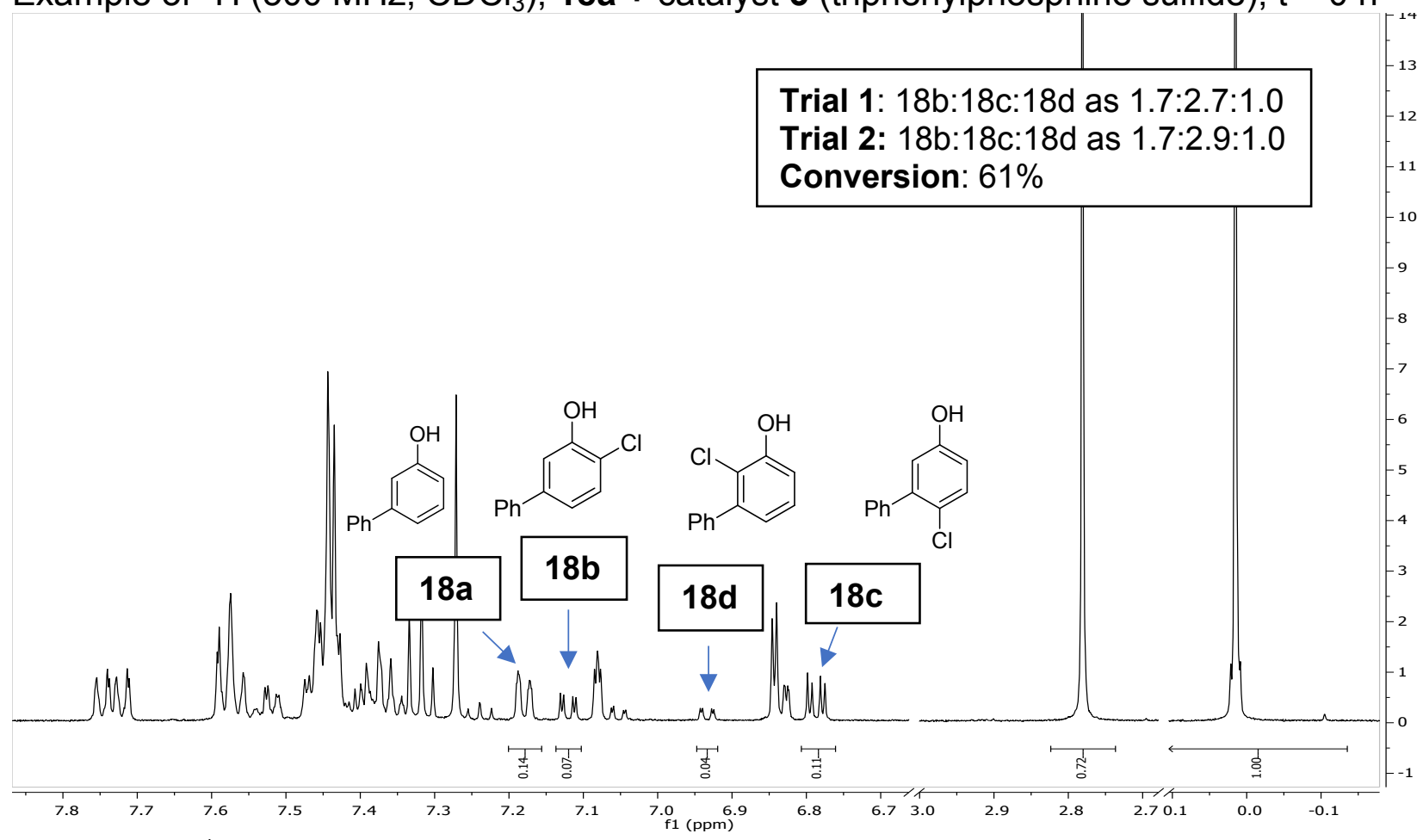

Example of ${ }^{1} \mathrm{H}\left(500 \mathrm{MHz}, \mathrm{CDCl}_{3}\right), 18 \mathrm{a}+$ catalyst 3 (triphenylphosphine sulfide); $\mathrm{t}=18 \mathrm{~h}$ 


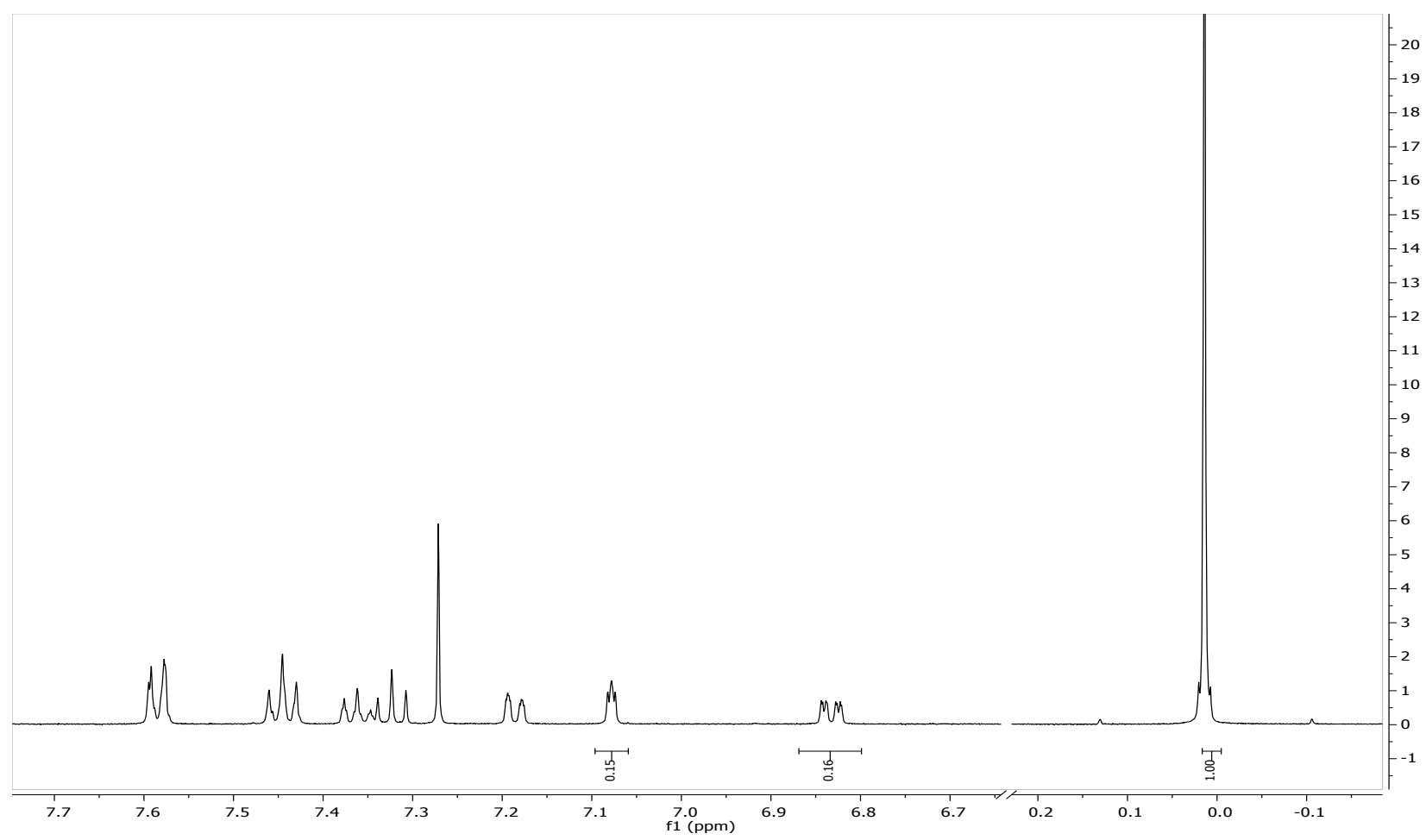

Example of ${ }^{1} \mathrm{H}\left(500 \mathrm{MHz}, \mathrm{CDCl}_{3}\right), \mathbf{1 8 a}+$ catalyst $10 ; \mathrm{t}=0 \mathrm{~h}$

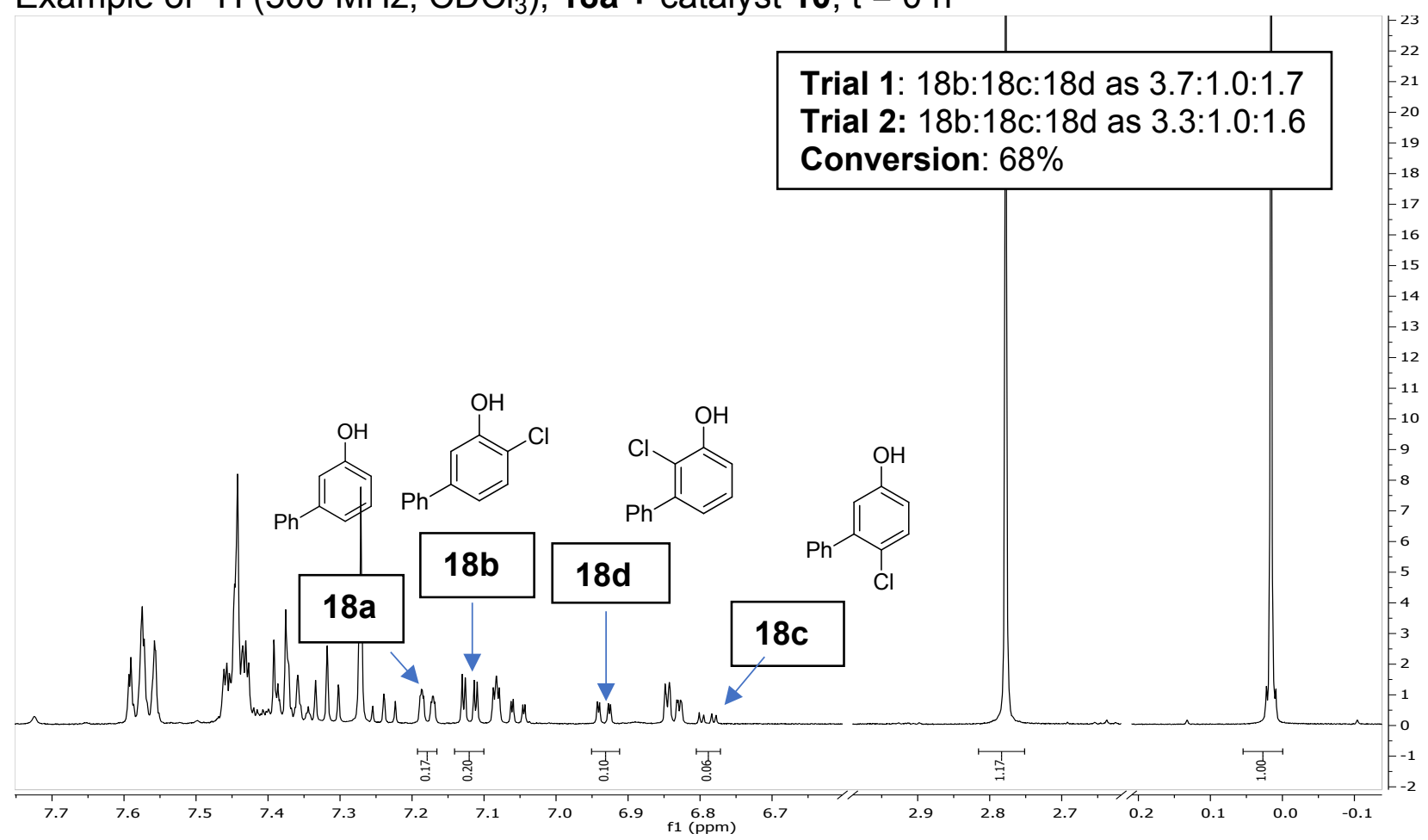

Example of ${ }^{1} \mathrm{H}\left(500 \mathrm{MHz}, \mathrm{CDCl}_{3}\right), \mathbf{1 8 a}+$ catalyst $10 ; \mathrm{t}=18 \mathrm{~h}$ 


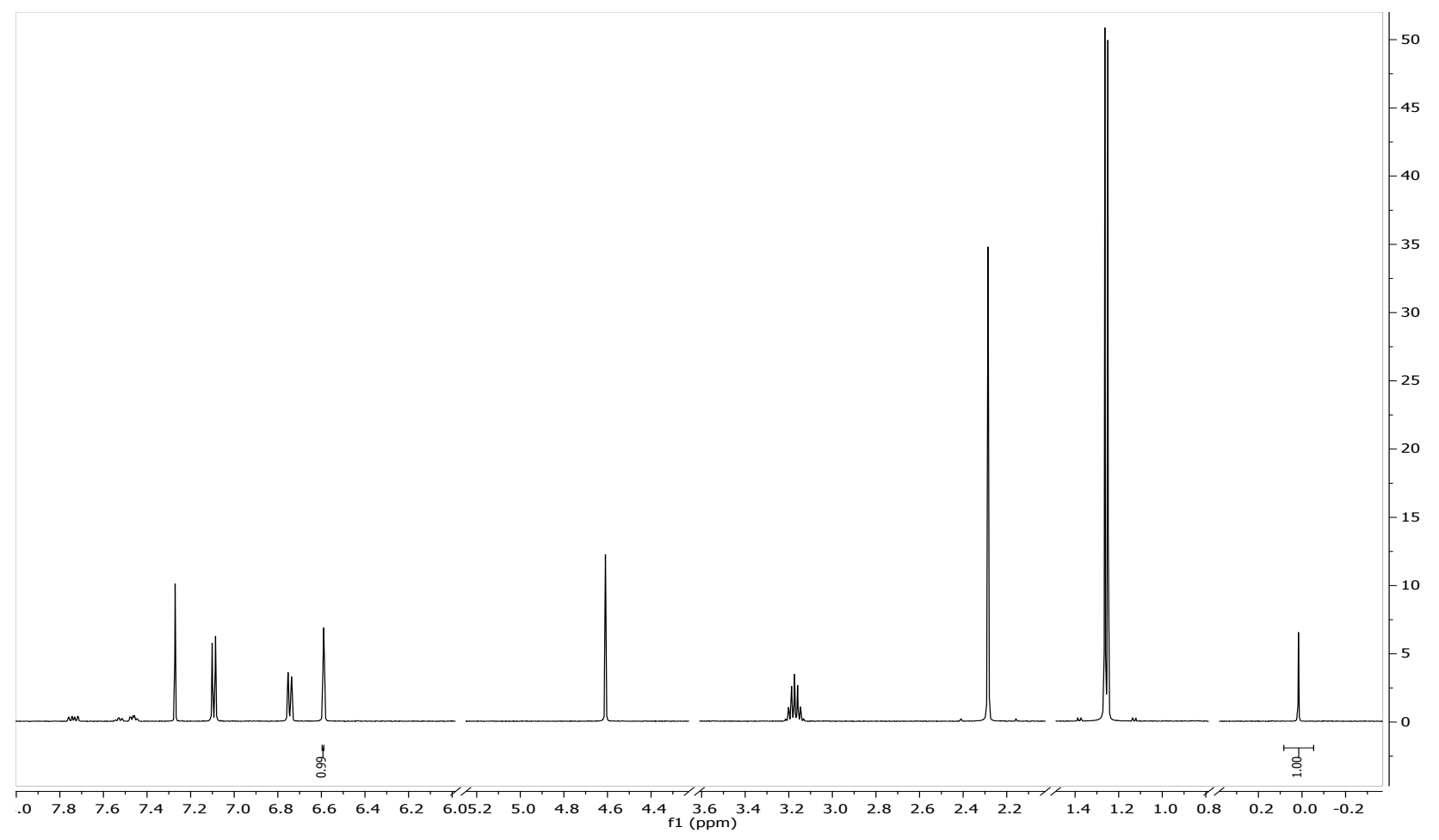

Example of ${ }^{1} \mathrm{H}\left(500 \mathrm{MHz}, \mathrm{CDCl}_{3}\right), 19 \mathrm{a}+$ catalyst 3 (triphenylphosphine sulfide); $\mathrm{t}=0 \mathrm{~h}$

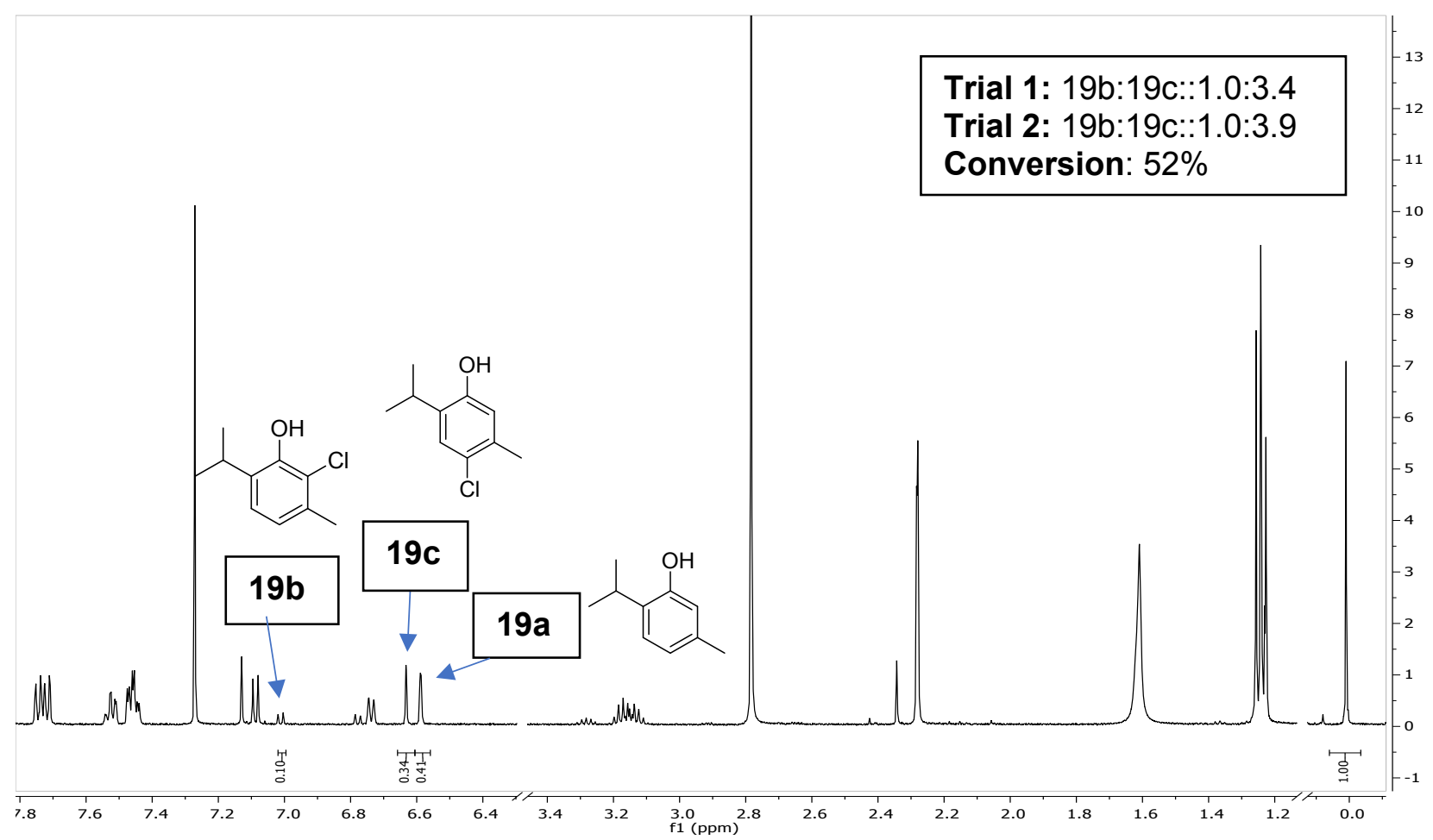

Example of ${ }^{1} \mathrm{H}\left(500 \mathrm{MHz}, \mathrm{CDCl}_{3}\right), 19 \mathrm{a}+$ catalyst 3 (triphenylphosphine sulfide); $\mathrm{t}=12 \mathrm{~h}$. 


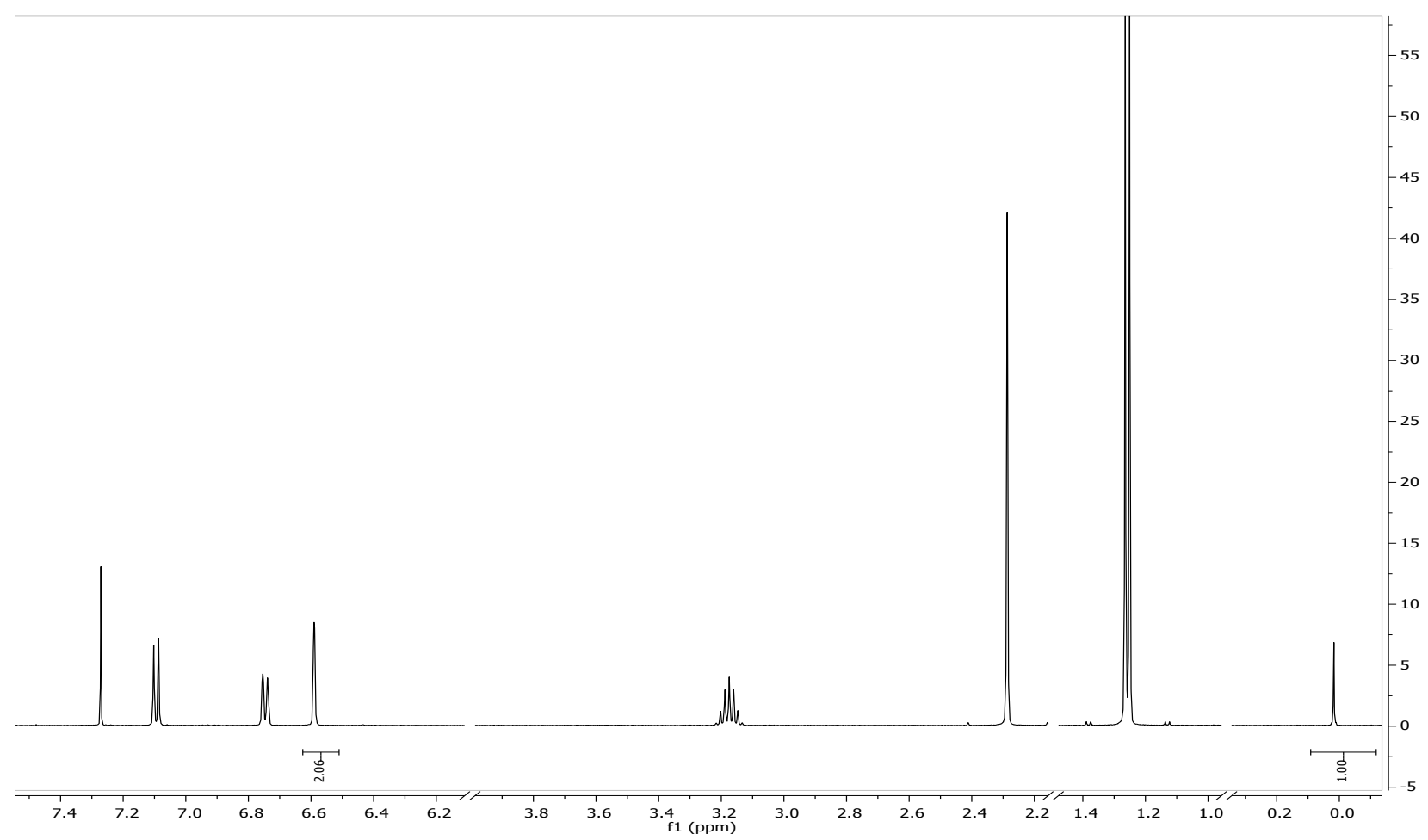

Example of ${ }^{1} \mathrm{H}\left(500 \mathrm{MHz}, \mathrm{CDCl}_{3}\right), 19 \mathrm{a}+$ catalyst $10 ; \mathrm{t}=0 \mathrm{~h}$

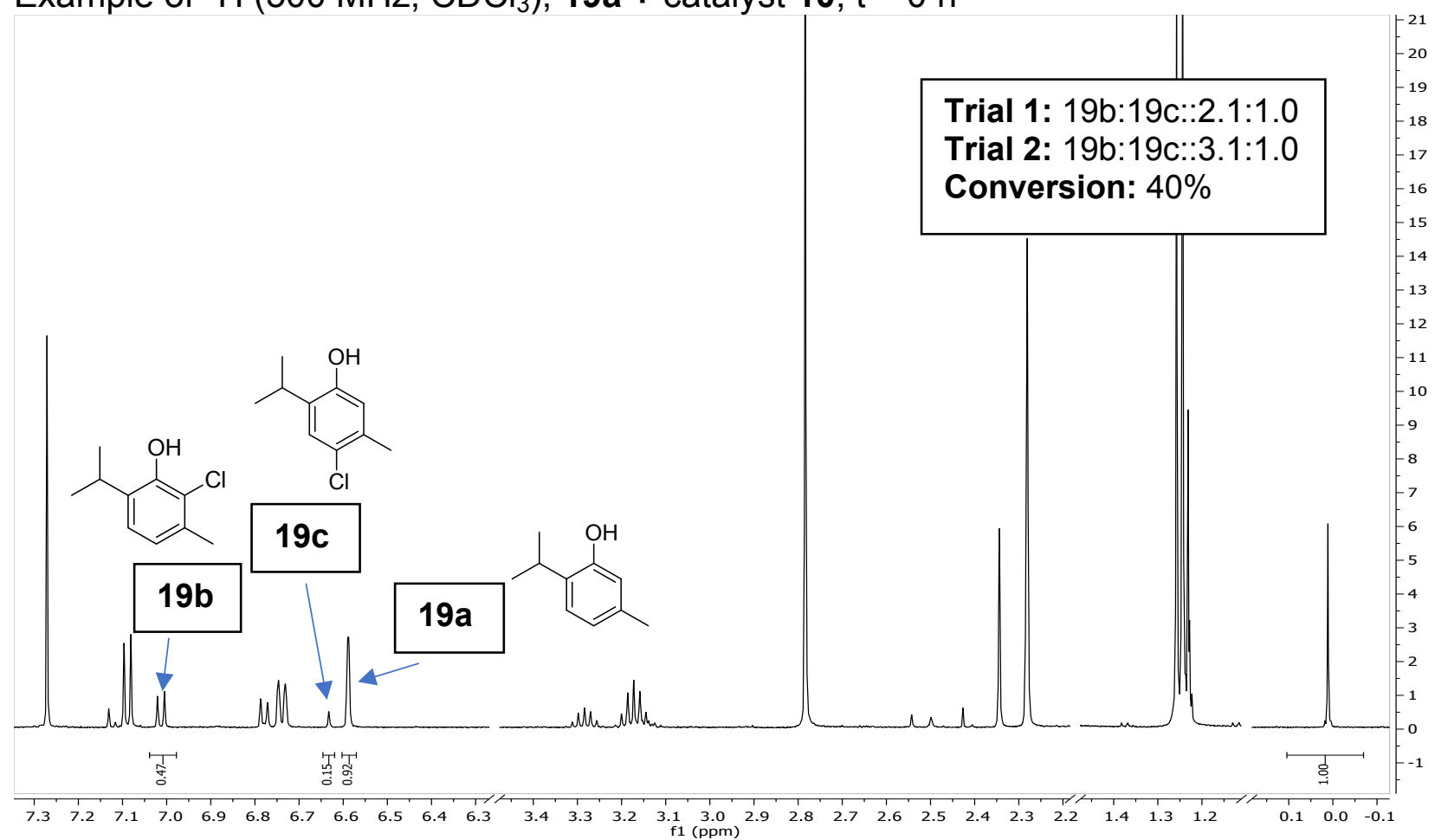

Example of ${ }^{1} \mathrm{H}\left(500 \mathrm{MHz}, \mathrm{CDCl}_{3}\right), 19 \mathrm{a}+$ catalyst $10 ; \mathrm{t}=12 \mathrm{~h}$ 


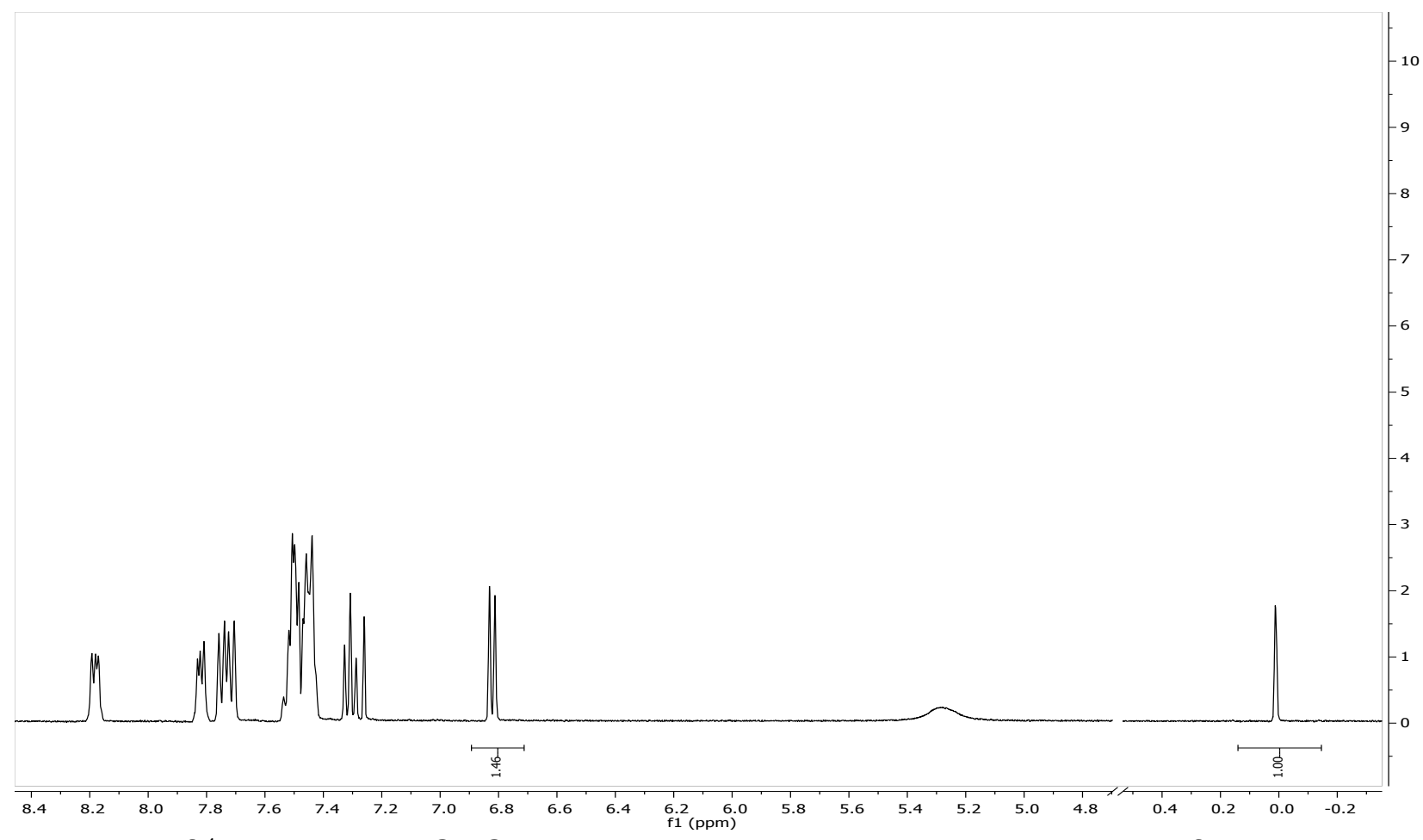

Example of ${ }^{1} \mathrm{H}\left(500 \mathrm{MHz}, \mathrm{CDCl}_{3}\right), 20 \mathrm{a}+$ catalyst 3 (triphenylphosphine sulfide); $\mathrm{t}=0 \mathrm{~h}$

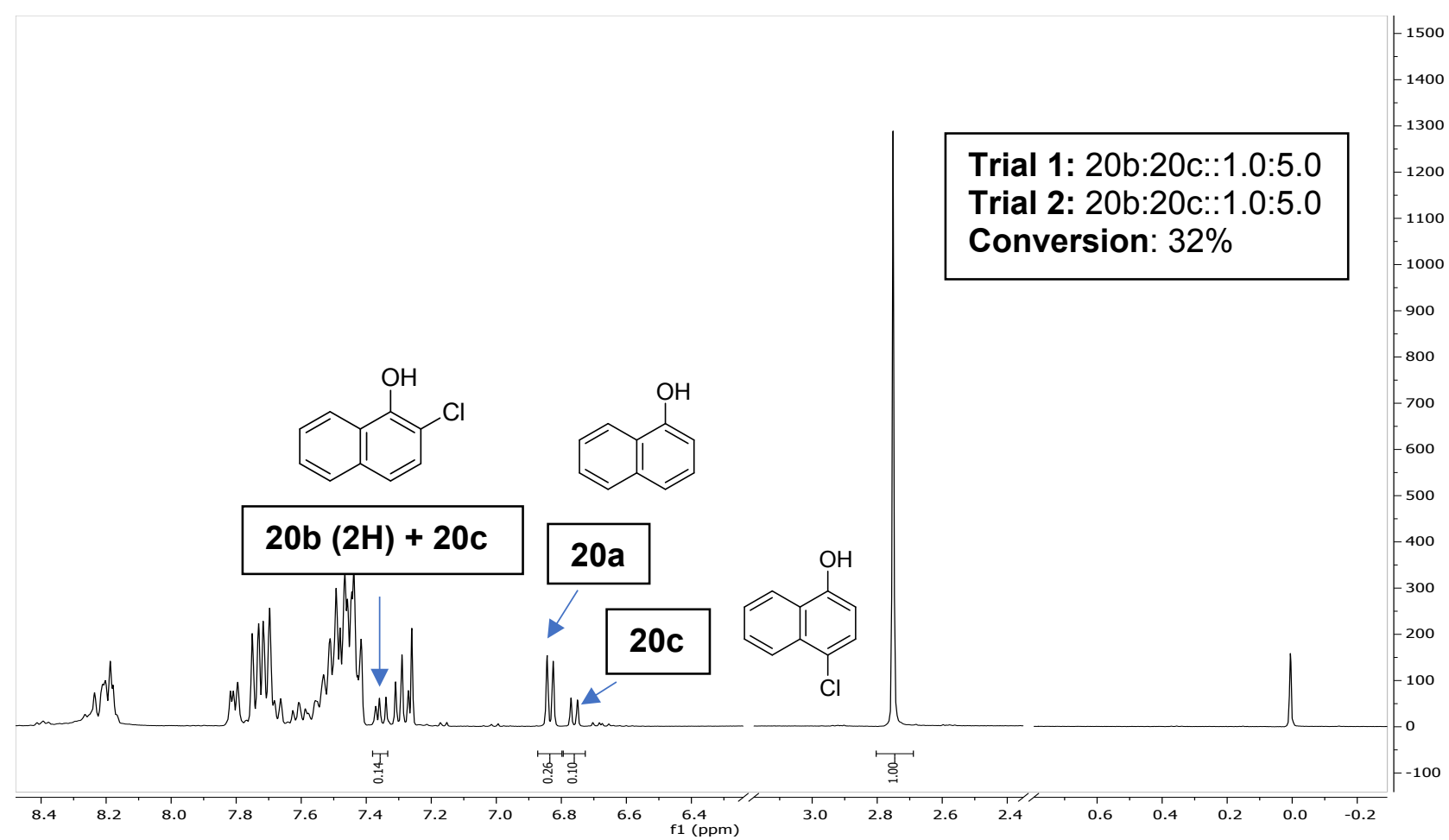

Example of ${ }^{1} \mathrm{H}\left(400 \mathrm{MHz}, \mathrm{CDCl}_{3}\right), 20 \mathrm{a}+$ catalyst 3 (triphenylphosphine sulfide); Full conversion based on succinimide and no presence of NCS. 


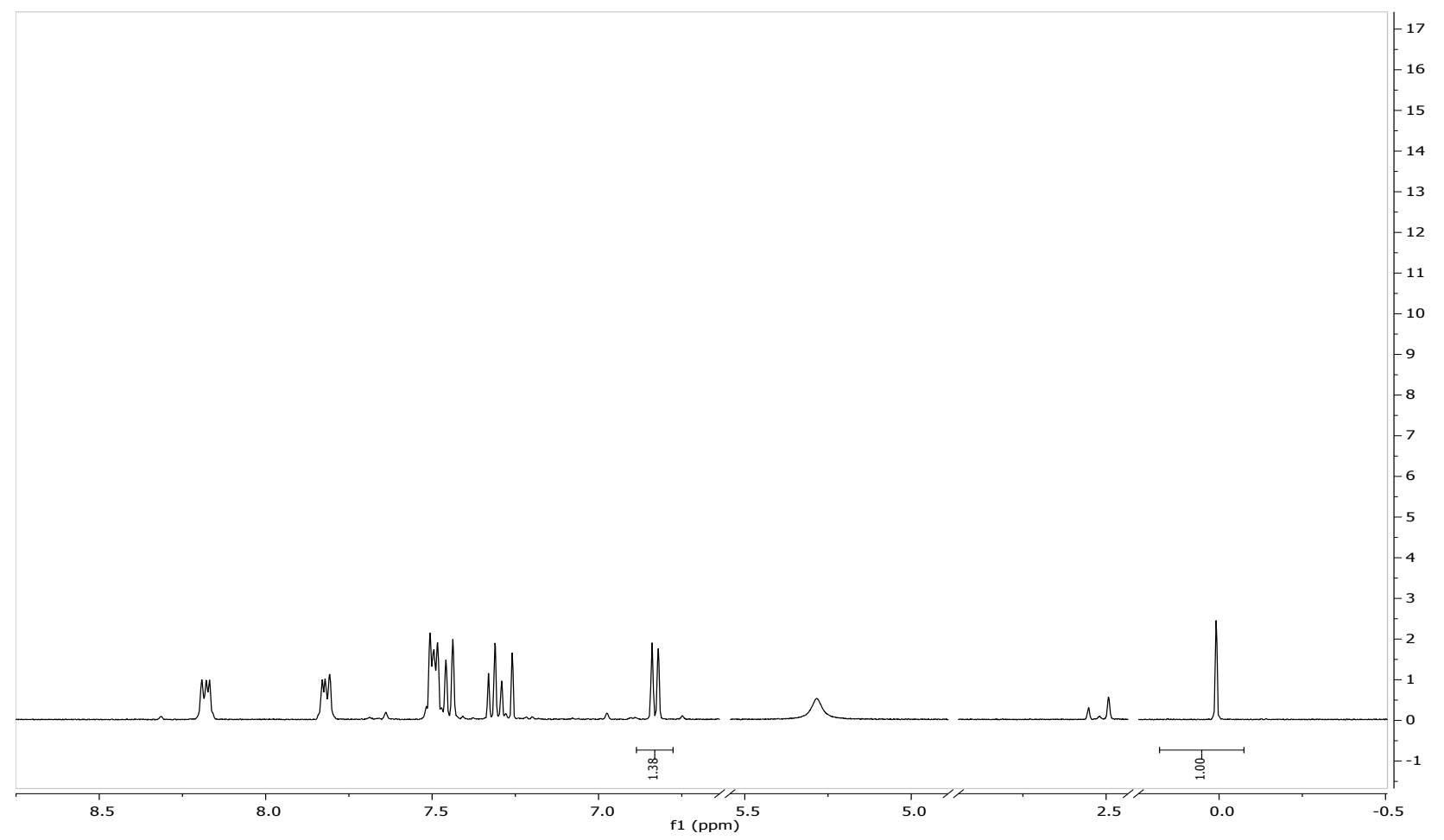

Example of ${ }^{1} \mathrm{H}\left(500 \mathrm{MHz}, \mathrm{CDCl}_{3}\right), 20 \mathrm{a}+$ catalyst $10 ; \mathrm{t}=0 \mathrm{~h}$

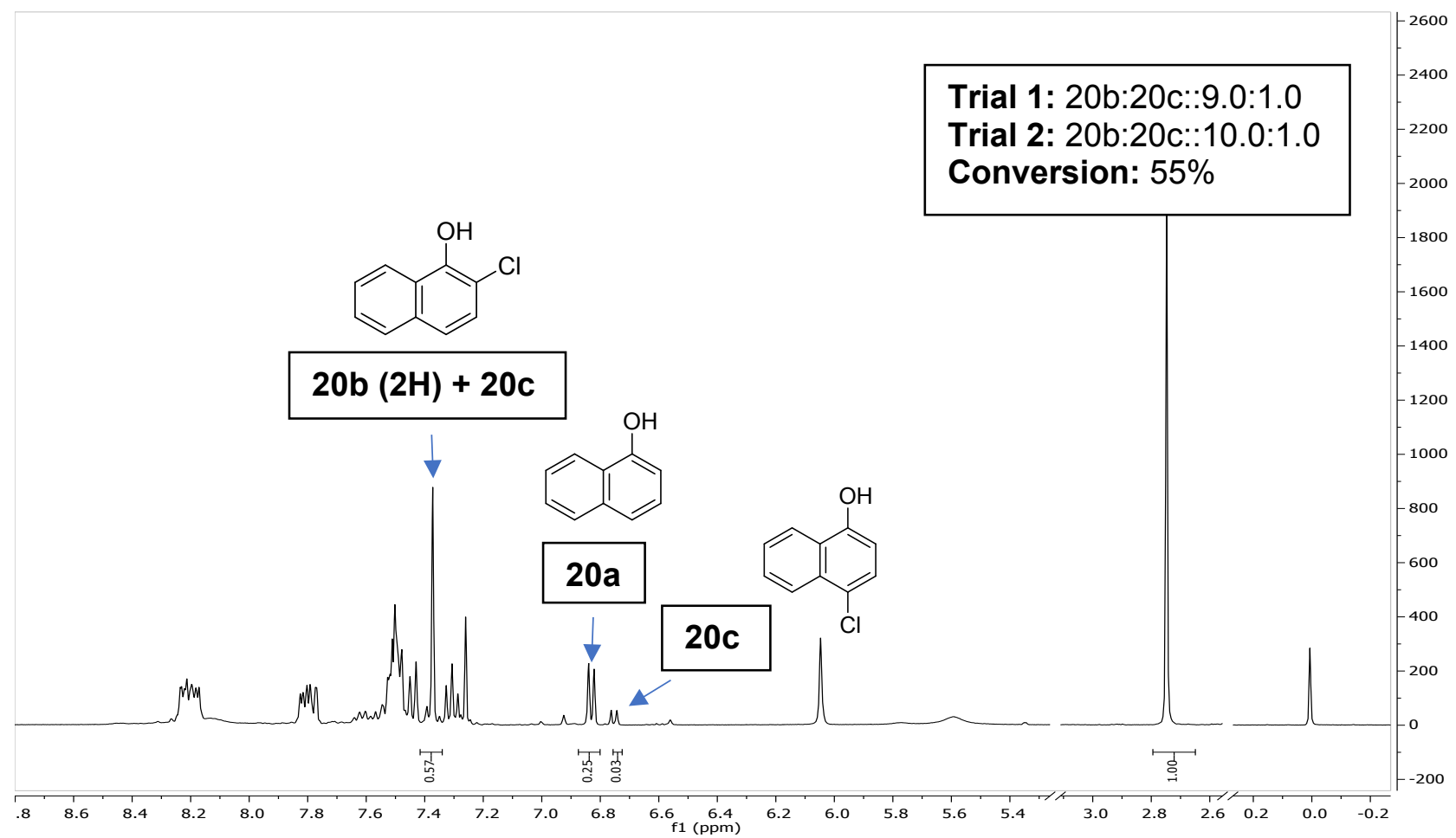

Example of ${ }^{1} \mathrm{H}\left(400 \mathrm{MHz}, \mathrm{CDCl}_{3}\right), 20 \mathrm{a}+$ catalyst $10 ; \mathrm{t}=12 \mathrm{~h}$. Full conversion based on succinimide and no presence of NCS. 


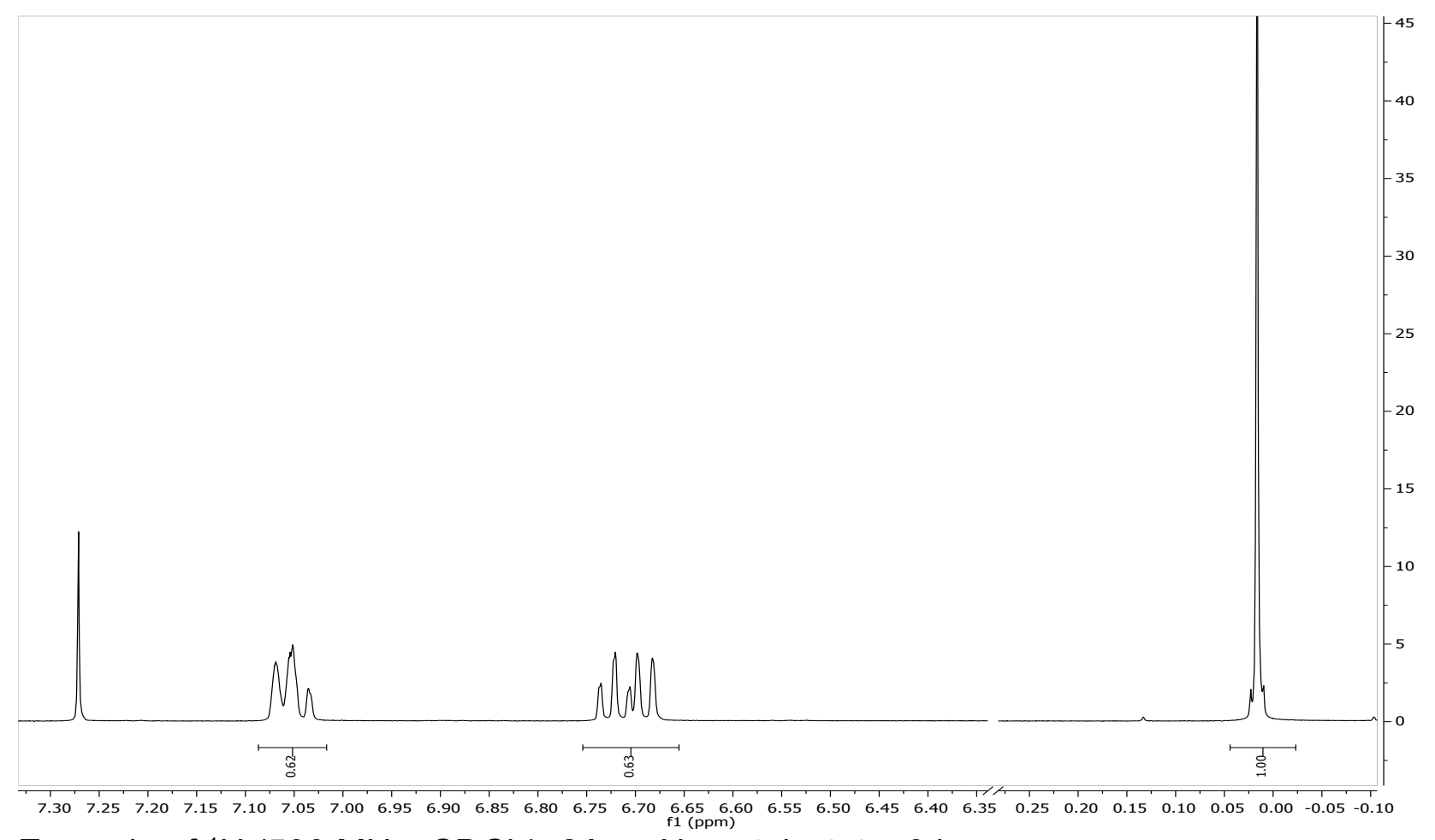

Example of ${ }^{1} \mathrm{H}\left(500 \mathrm{MHz}, \mathrm{CDCl}_{3}\right), 21 \mathrm{a}+$ No catalyst; $\mathrm{t}=0 \mathrm{~h}$

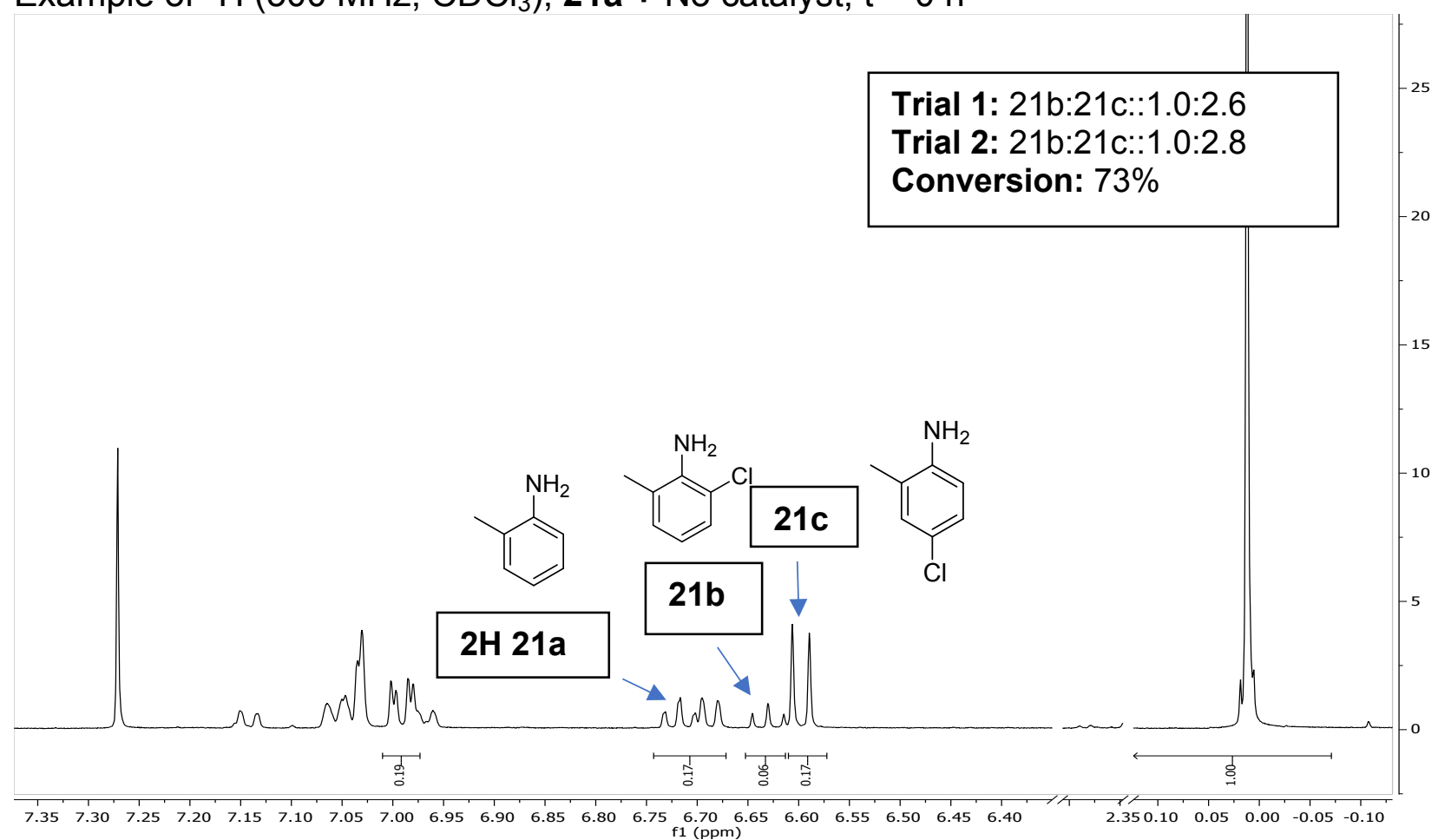

Example of ${ }^{1} \mathrm{H}\left(500 \mathrm{MHz}, \mathrm{CDCl}_{3}\right), 21 \mathrm{a}+$ No catalyst; $\mathrm{t}=12 \mathrm{~h}$ 


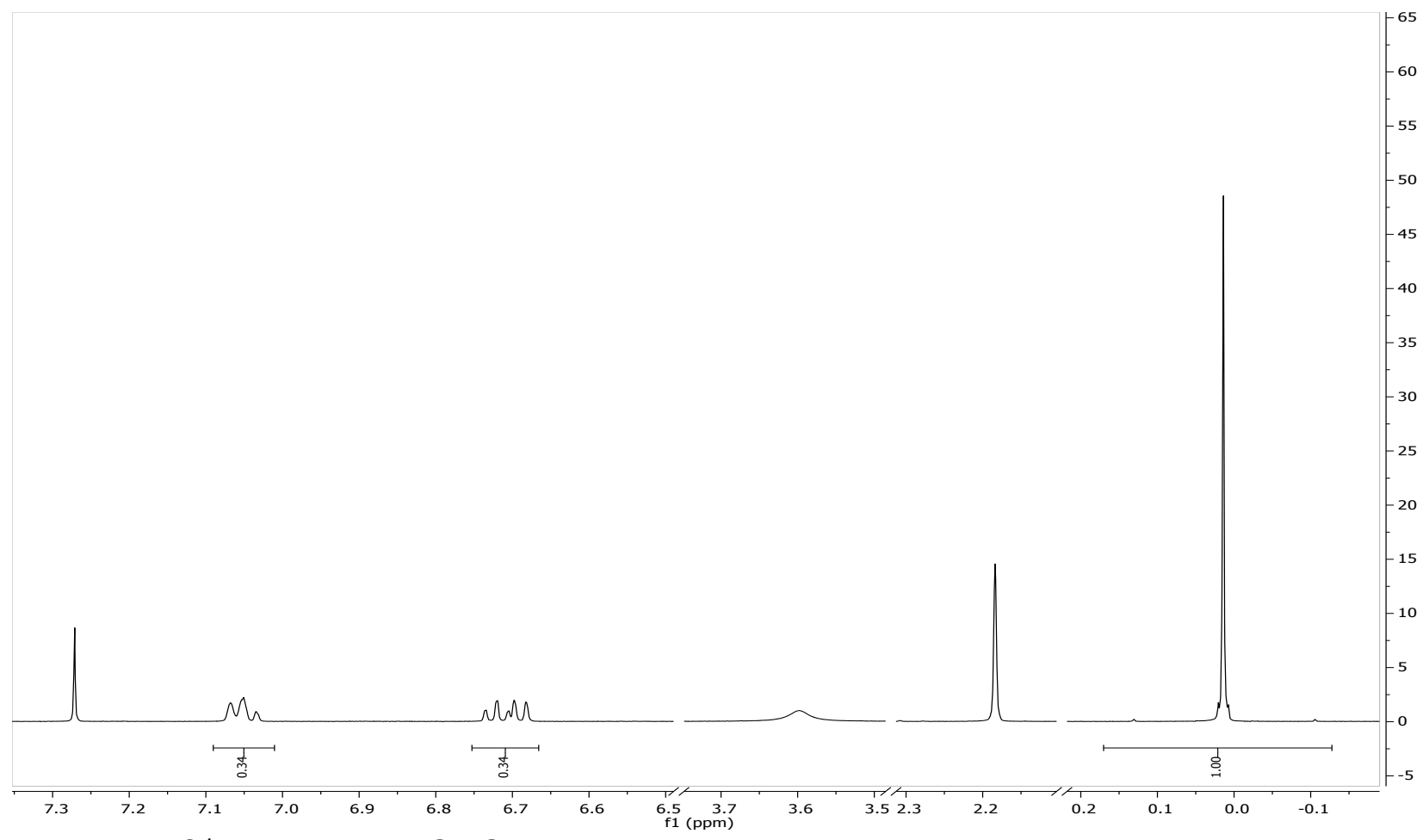

Example of ${ }^{1} \mathrm{H}\left(500 \mathrm{MHz}, \mathrm{CDCl}_{3}\right), 21 \mathrm{a}+$ catalyst $10 ; \mathrm{t}=0 \mathrm{~h}$

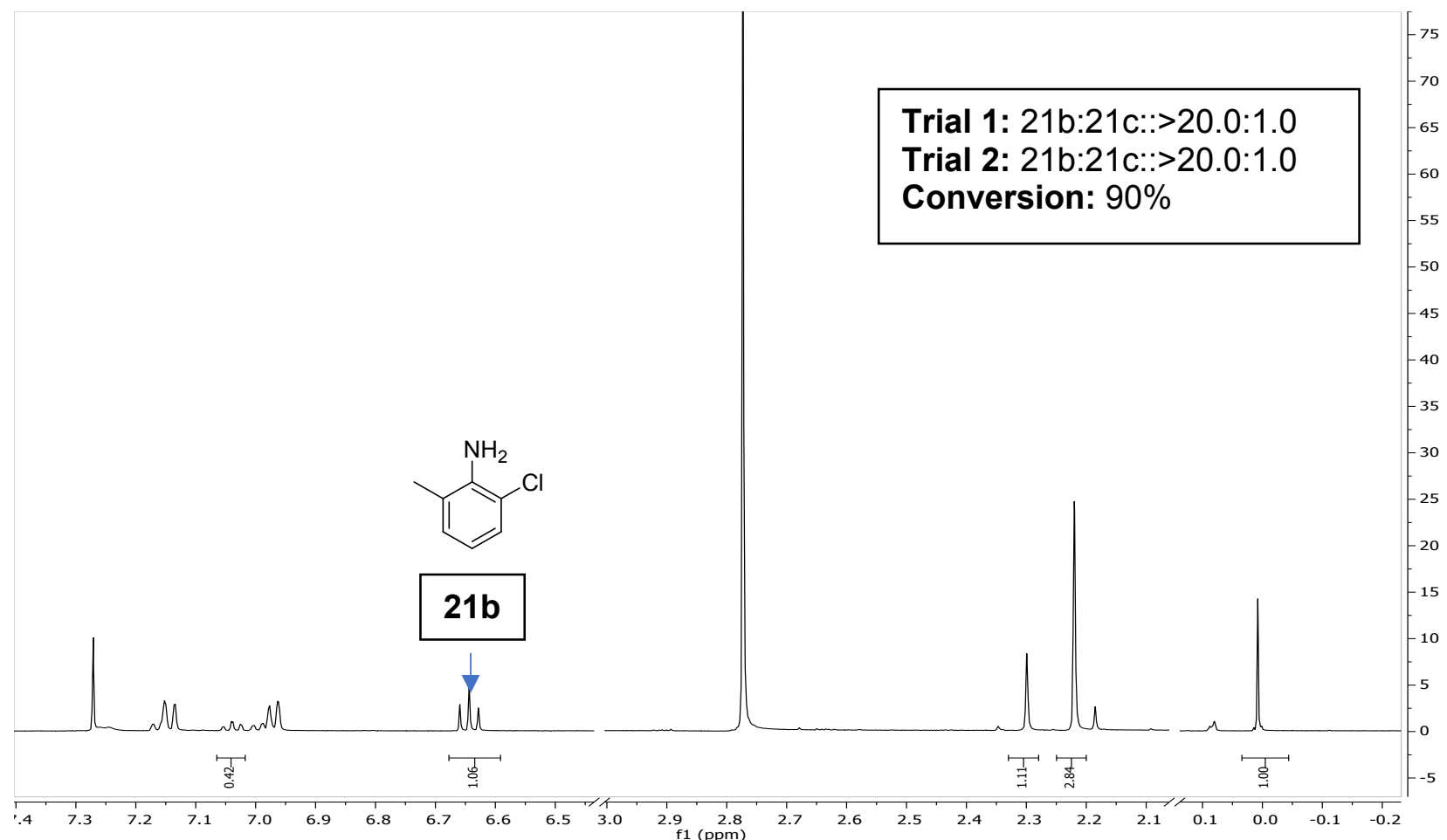

Example of ${ }^{1} \mathrm{H}\left(500 \mathrm{MHz}, \mathrm{CDCl}_{3}\right), 21 \mathrm{a}+$ catalyst 10; $\mathrm{t}=12 \mathrm{~h}$. There is no presence of 21a or 21c but another ortho-substituted byproduct. This is believed to be a chloronium salt. 


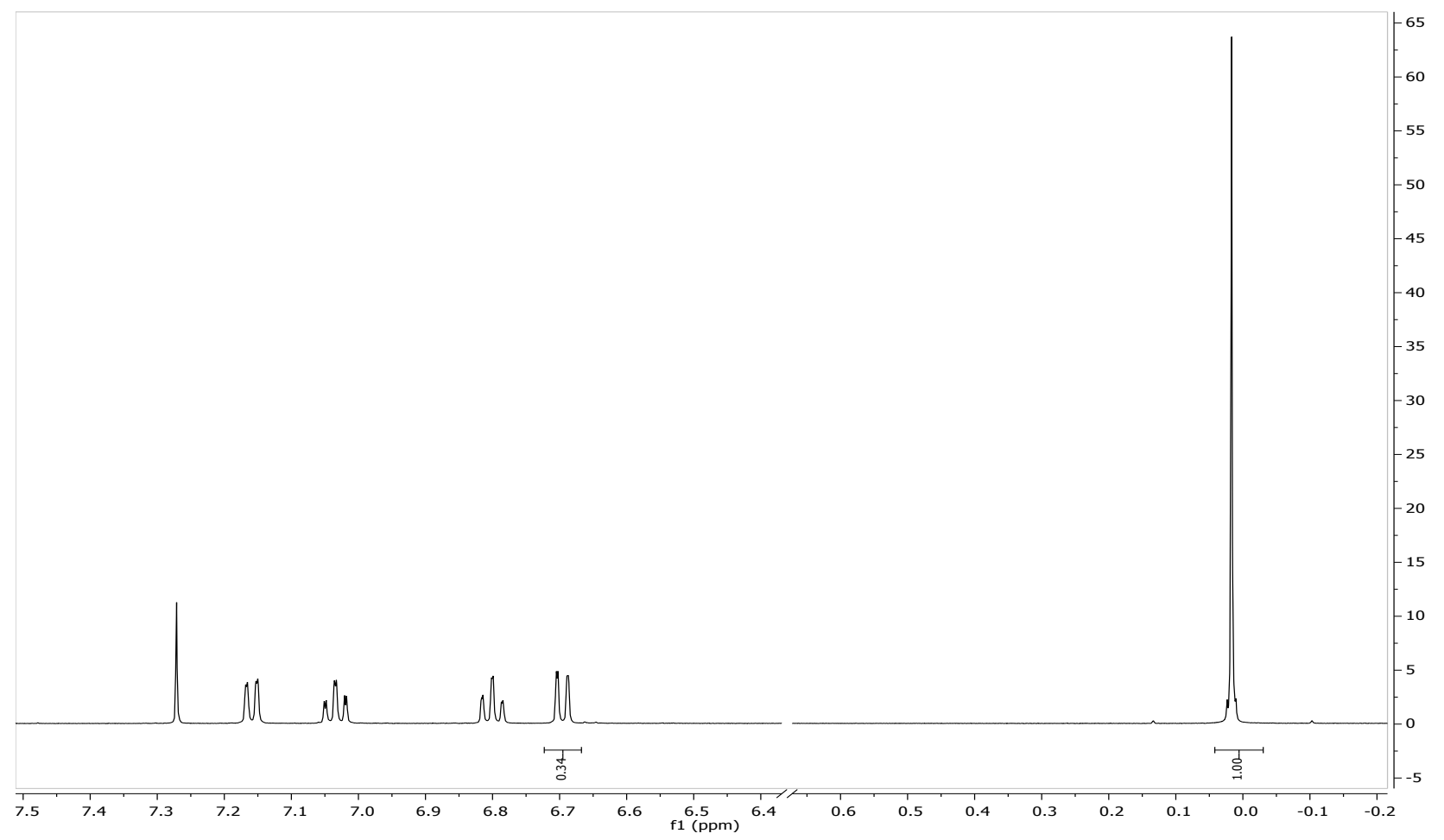

Example of ${ }^{1} \mathrm{H}\left(500 \mathrm{MHz}, \mathrm{CDCl}_{3}\right), 22 \mathrm{a}+$ No catalyst; $\mathrm{t}=0 \mathrm{~h}$

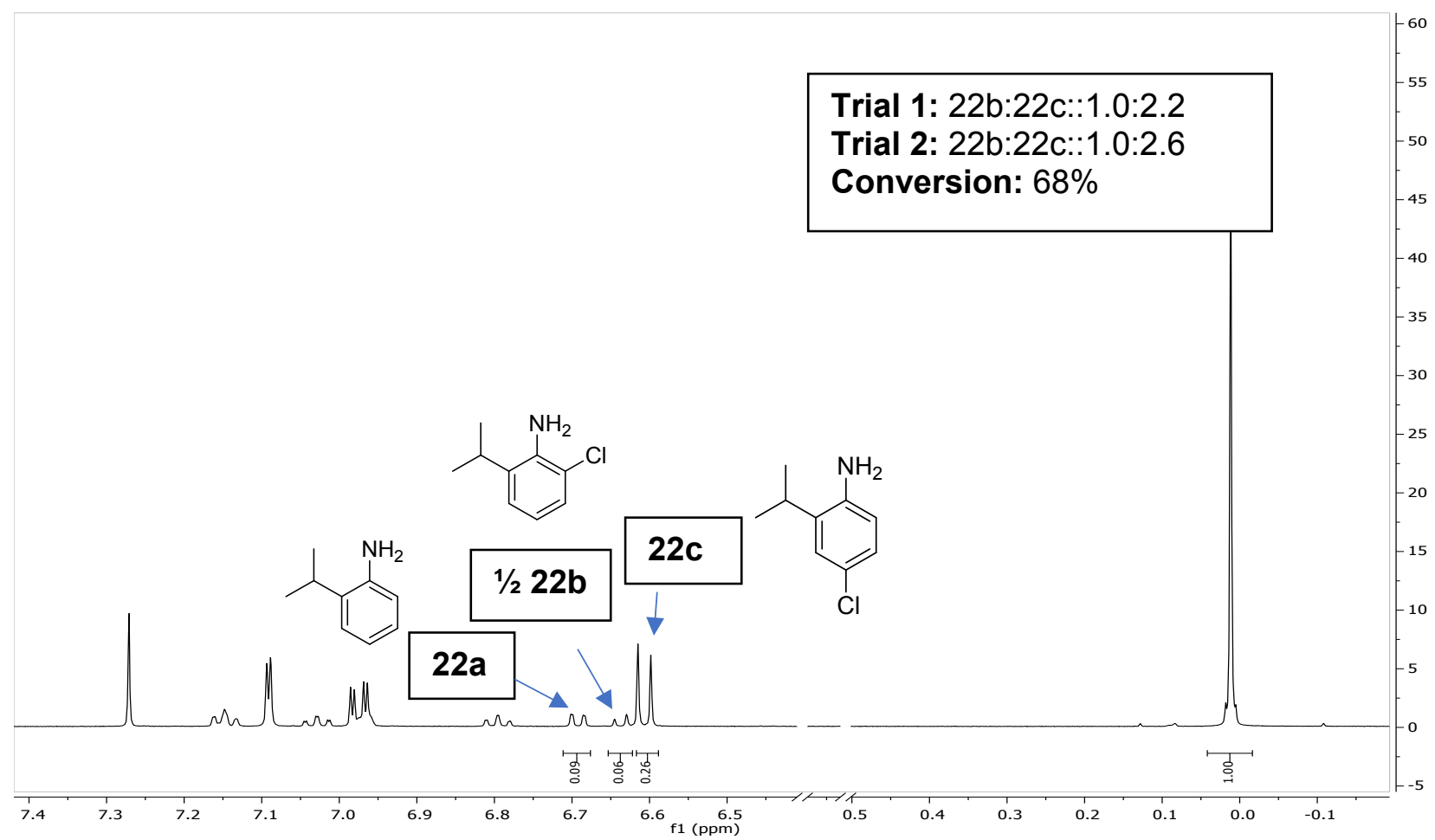

Example of ${ }^{1} \mathrm{H}\left(500 \mathrm{MHz}, \mathrm{CDCl}_{3}\right), 22 \mathrm{a}+$ No catalyst; $\mathrm{t}=12 \mathrm{~h}$ 


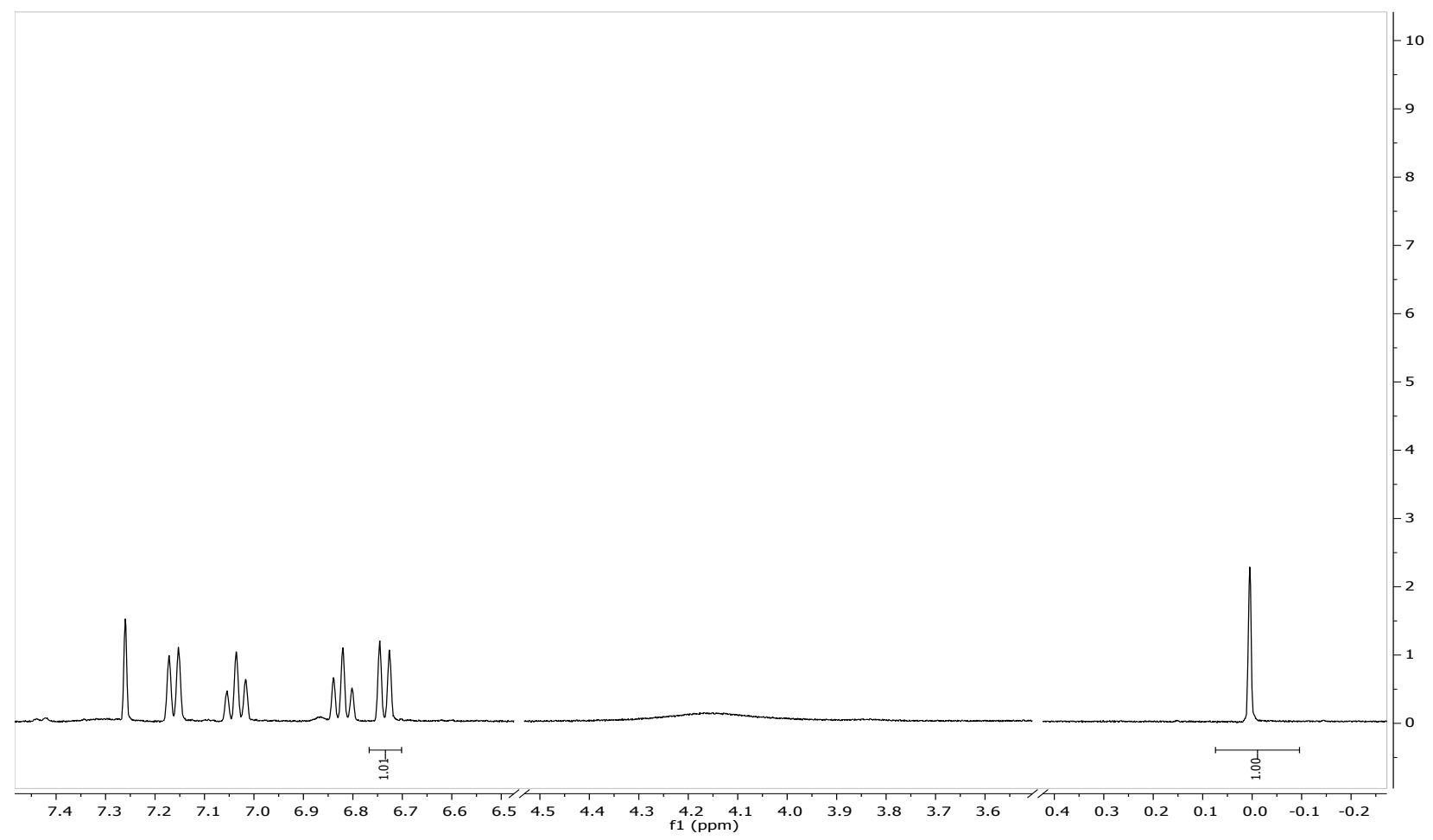

Example of ${ }^{1} \mathrm{H}\left(500 \mathrm{MHz}, \mathrm{CDCl}_{3}\right), 22 \mathrm{a}+$ catalyst $10 ; \mathrm{t}=0 \mathrm{~h}$

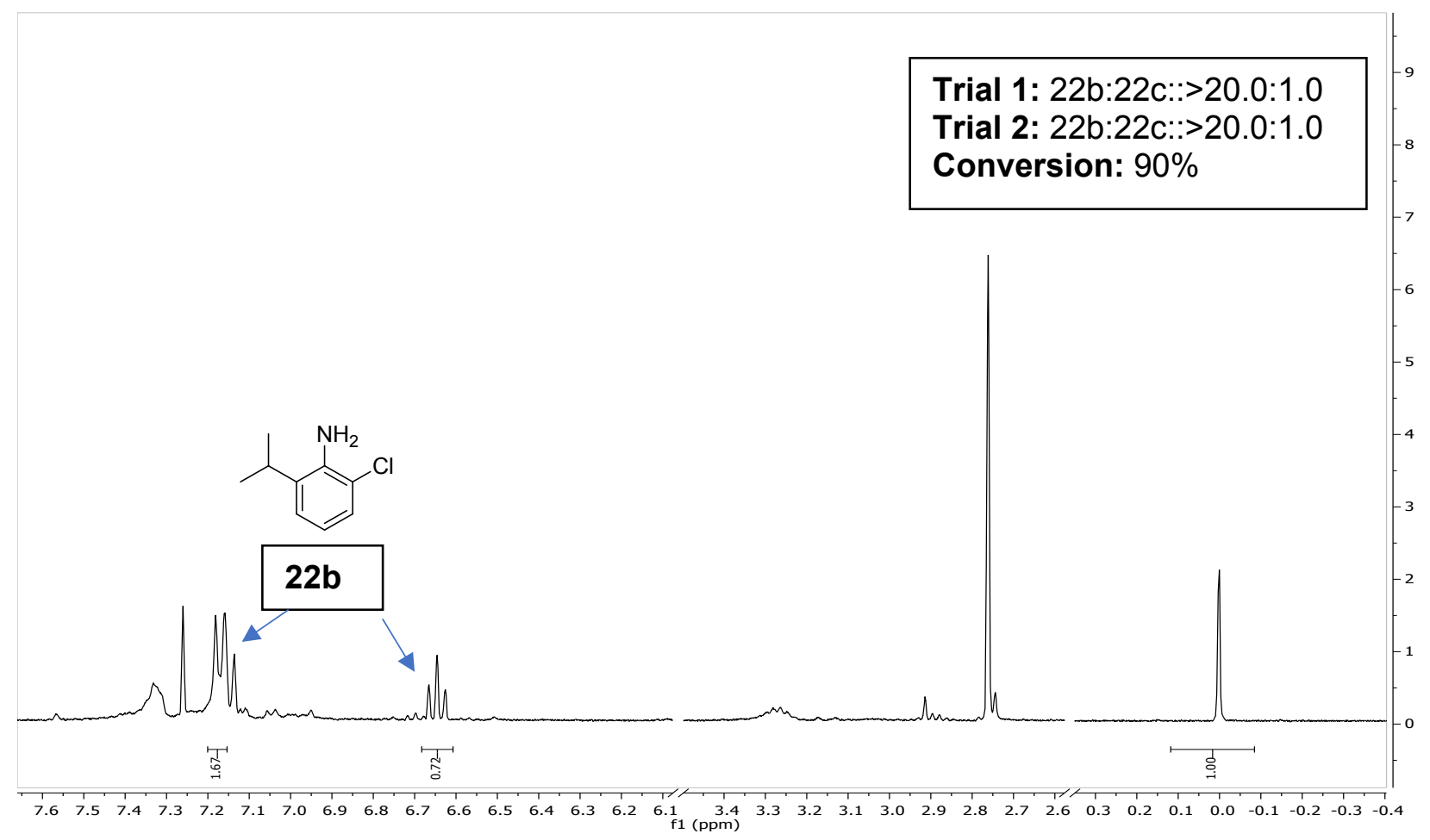

Example of ${ }^{1} \mathrm{H}\left(500 \mathrm{MHz}, \mathrm{CDCl}_{3}\right), 22 \mathrm{a}+$ catalyst $10 ; \mathrm{t}=12 \mathrm{~h}$. There is no presence of 21a or 21c. 


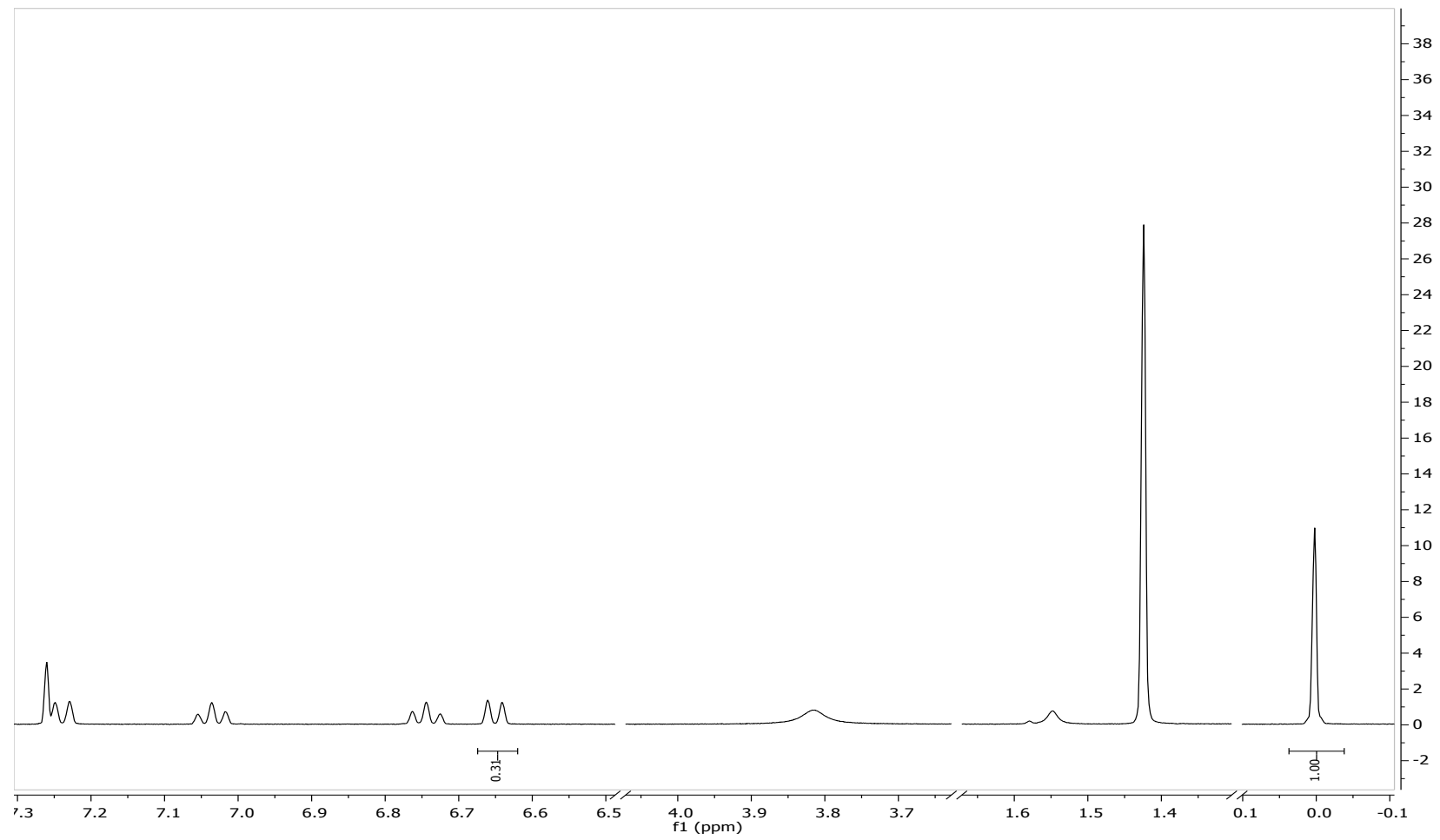

Example of ${ }^{1} \mathrm{H}\left(500 \mathrm{MHz}, \mathrm{CDCl}_{3}\right), 23 \mathrm{a}+$ No catalyst; $\mathrm{t}=0 \mathrm{~h}$

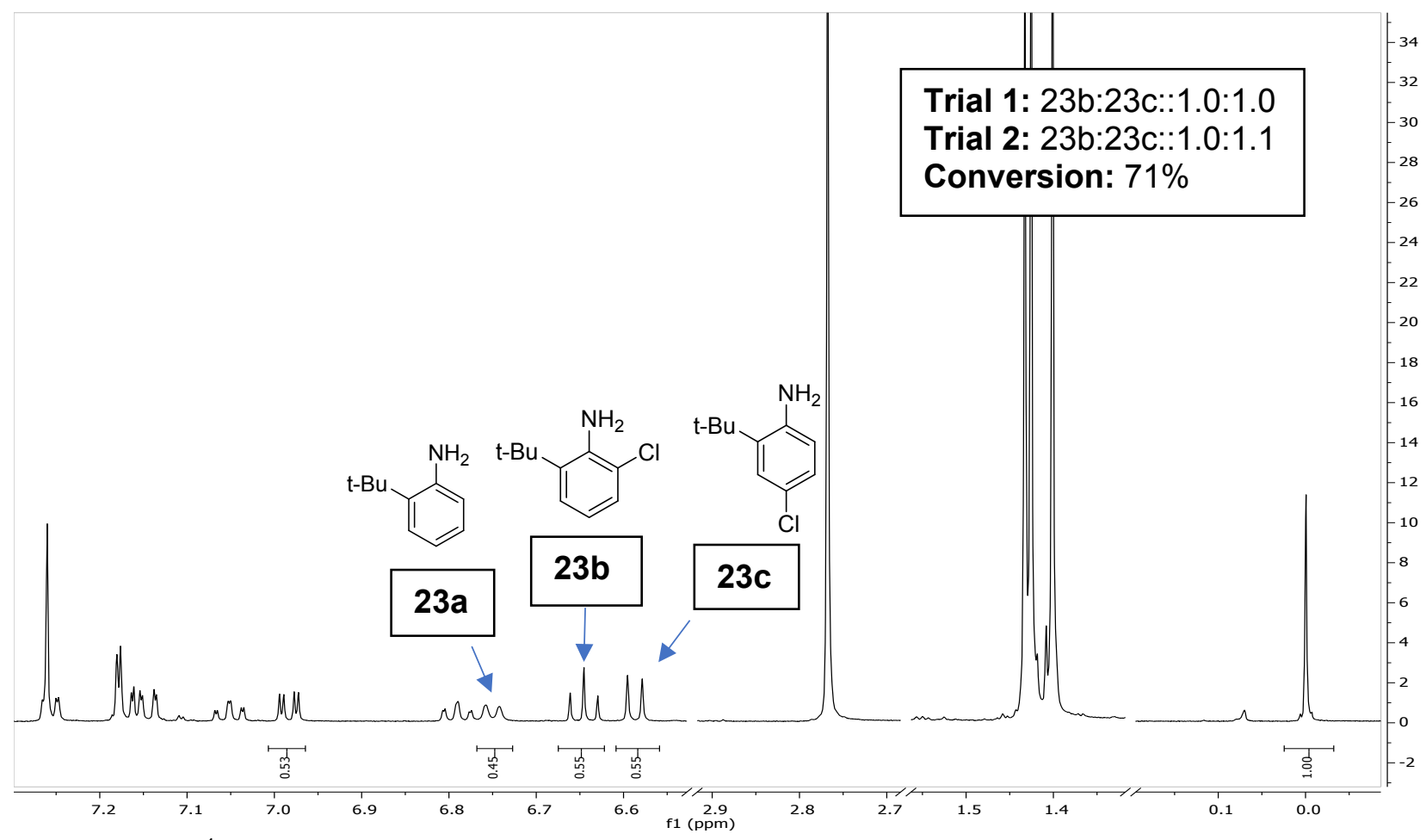

Example of ${ }^{1} \mathrm{H}\left(500 \mathrm{MHz}, \mathrm{CDCl}_{3}\right), 23 \mathrm{a}+$ No catalyst; $\mathrm{t}=12 \mathrm{~h}$ 


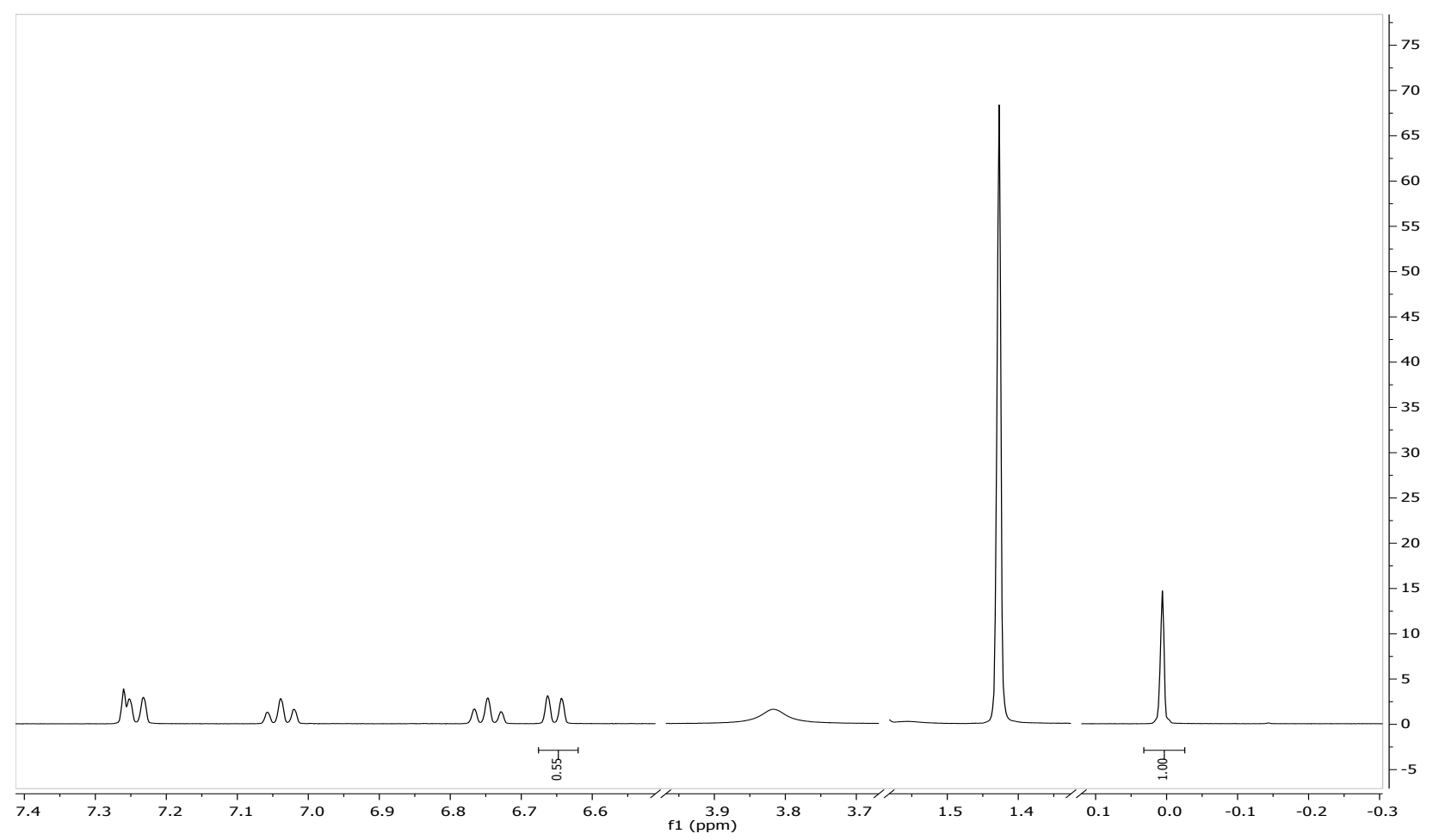

Example of ${ }^{1} \mathrm{H}\left(500 \mathrm{MHz}, \mathrm{CDCl}_{3}\right), 23 \mathrm{a}+$ catalyst $10 ; \mathrm{t}=0 \mathrm{~h}$

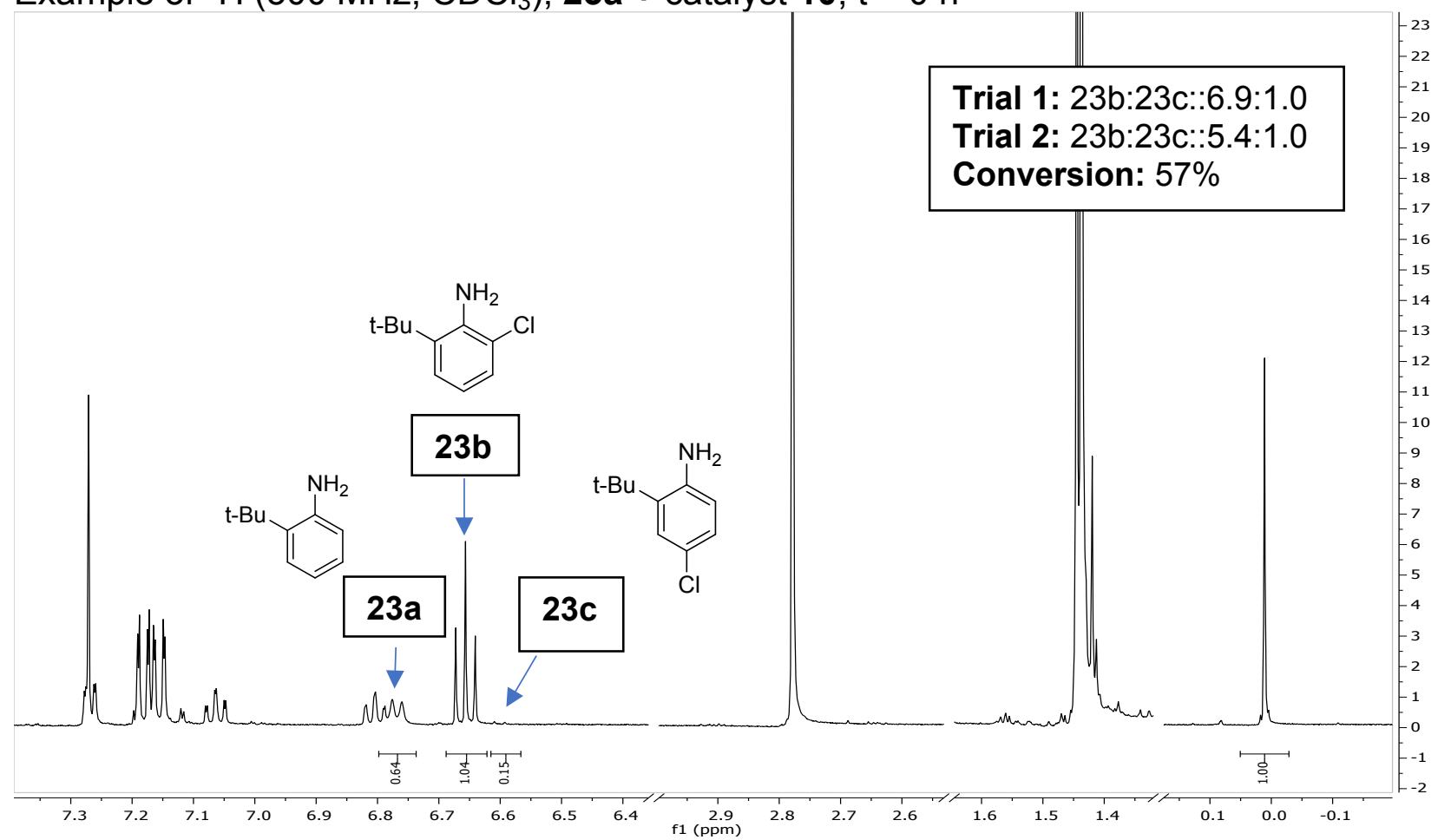

Example of ${ }^{1} \mathrm{H}\left(500 \mathrm{MHz}, \mathrm{CDCl}_{3}\right), 23 \mathrm{a}+$ catalyst $10 ; \mathrm{t}=12 \mathrm{~h}$ 


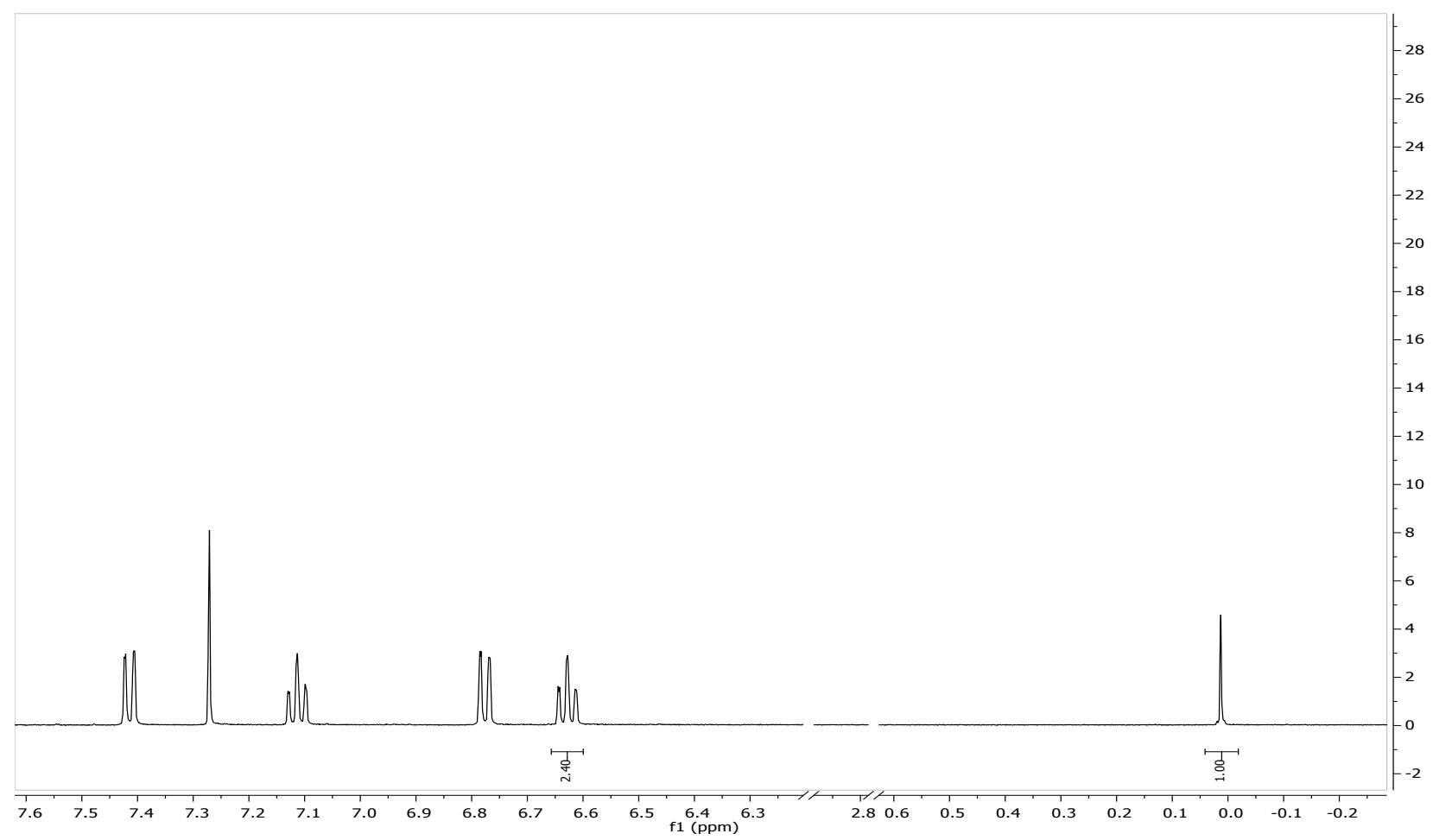

Example of ${ }^{1} \mathrm{H}\left(500 \mathrm{MHz}, \mathrm{CDCl}_{3}\right), 24 \mathrm{a}+$ No catalyst; $\mathrm{t}=0 \mathrm{~h}$

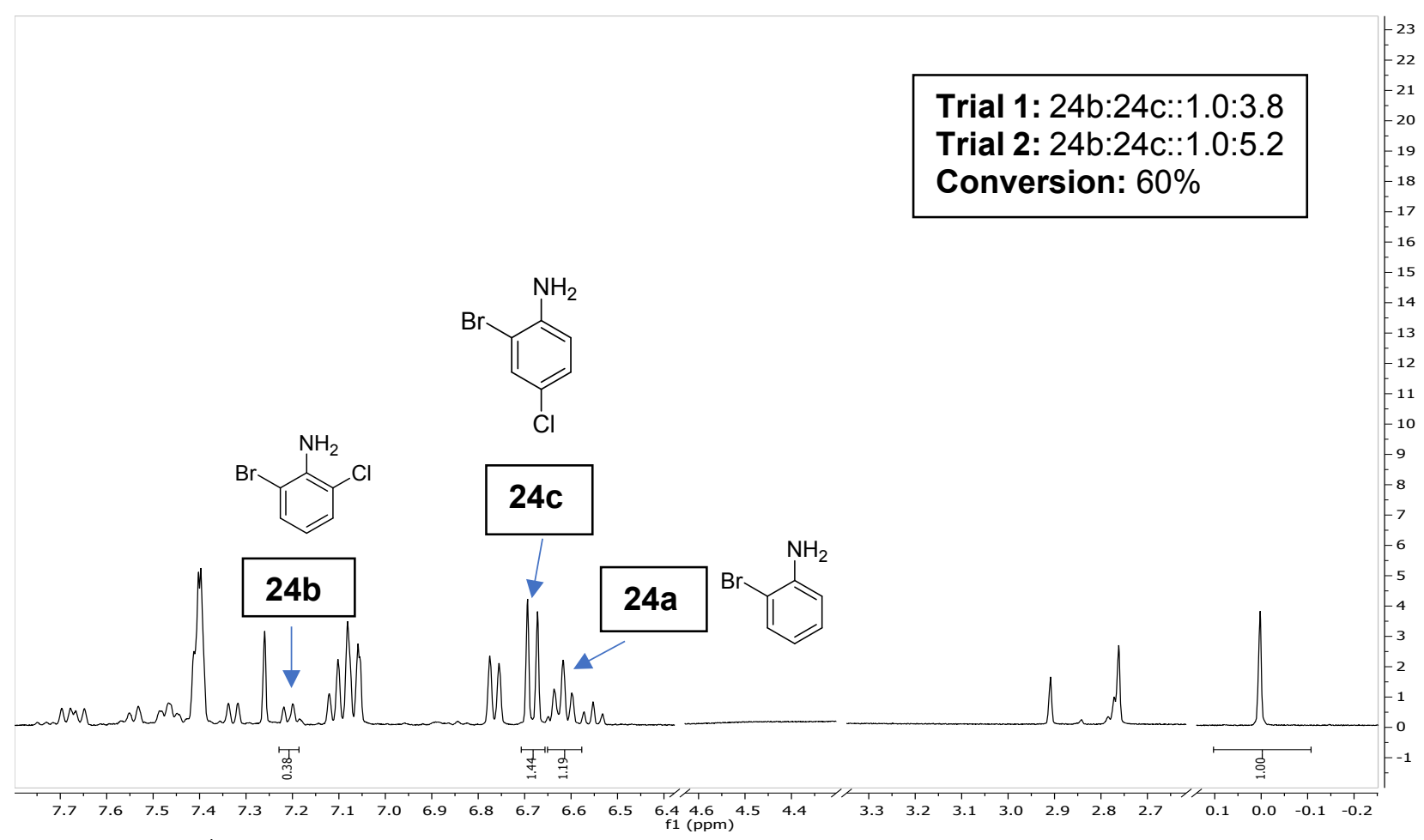

Example of ${ }^{1} \mathrm{H}\left(500 \mathrm{MHz}, \mathrm{CDCl}_{3}\right), 24 \mathrm{a}+$ No catalyst; $\mathrm{t}=18 \mathrm{~h}$ 


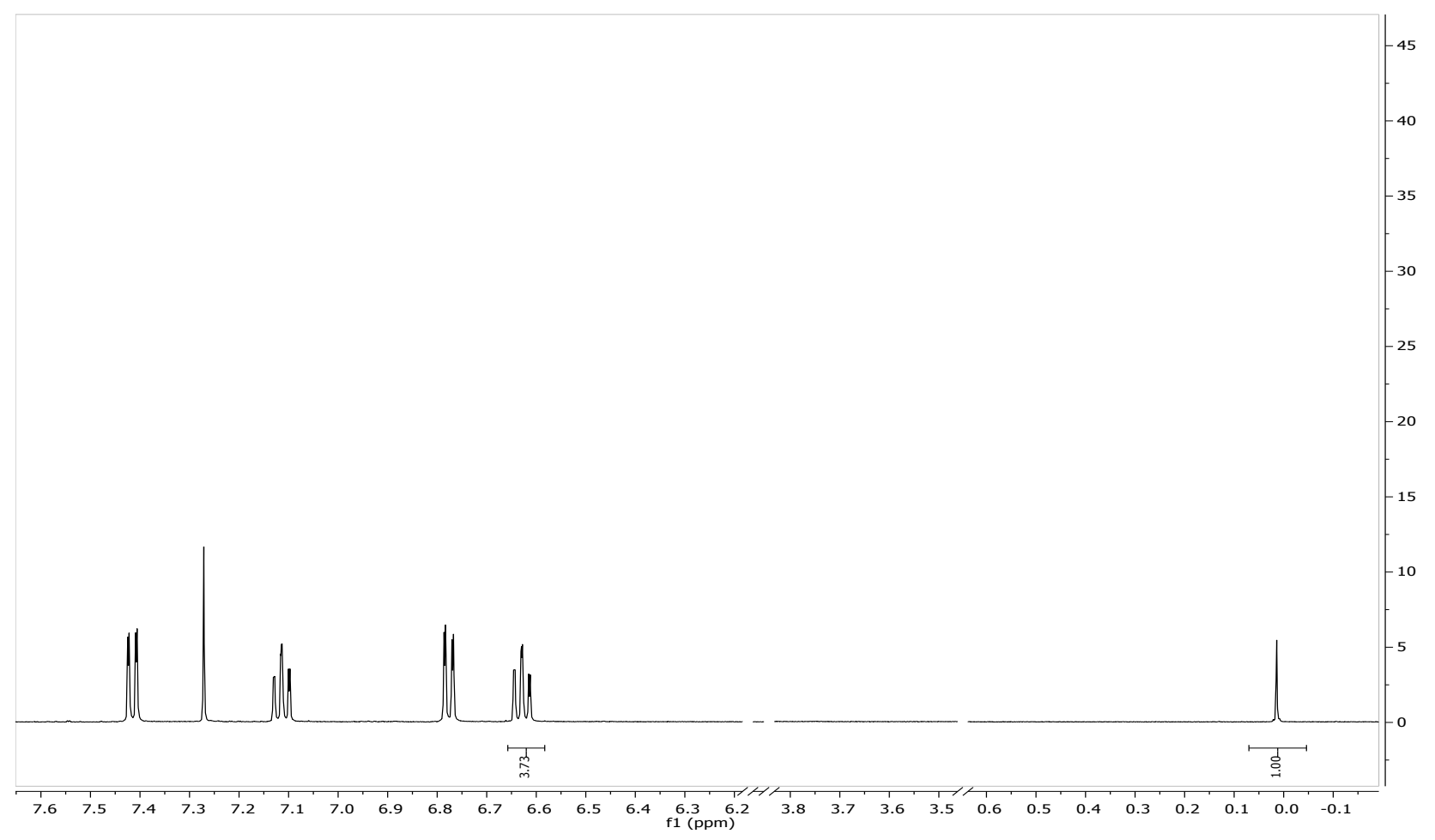

Example of ${ }^{1} \mathrm{H}\left(500 \mathrm{MHz}, \mathrm{CDCl}_{3}\right), 24 \mathrm{a}+$ catalyst $10 ; \mathrm{t}=0 \mathrm{~h}$

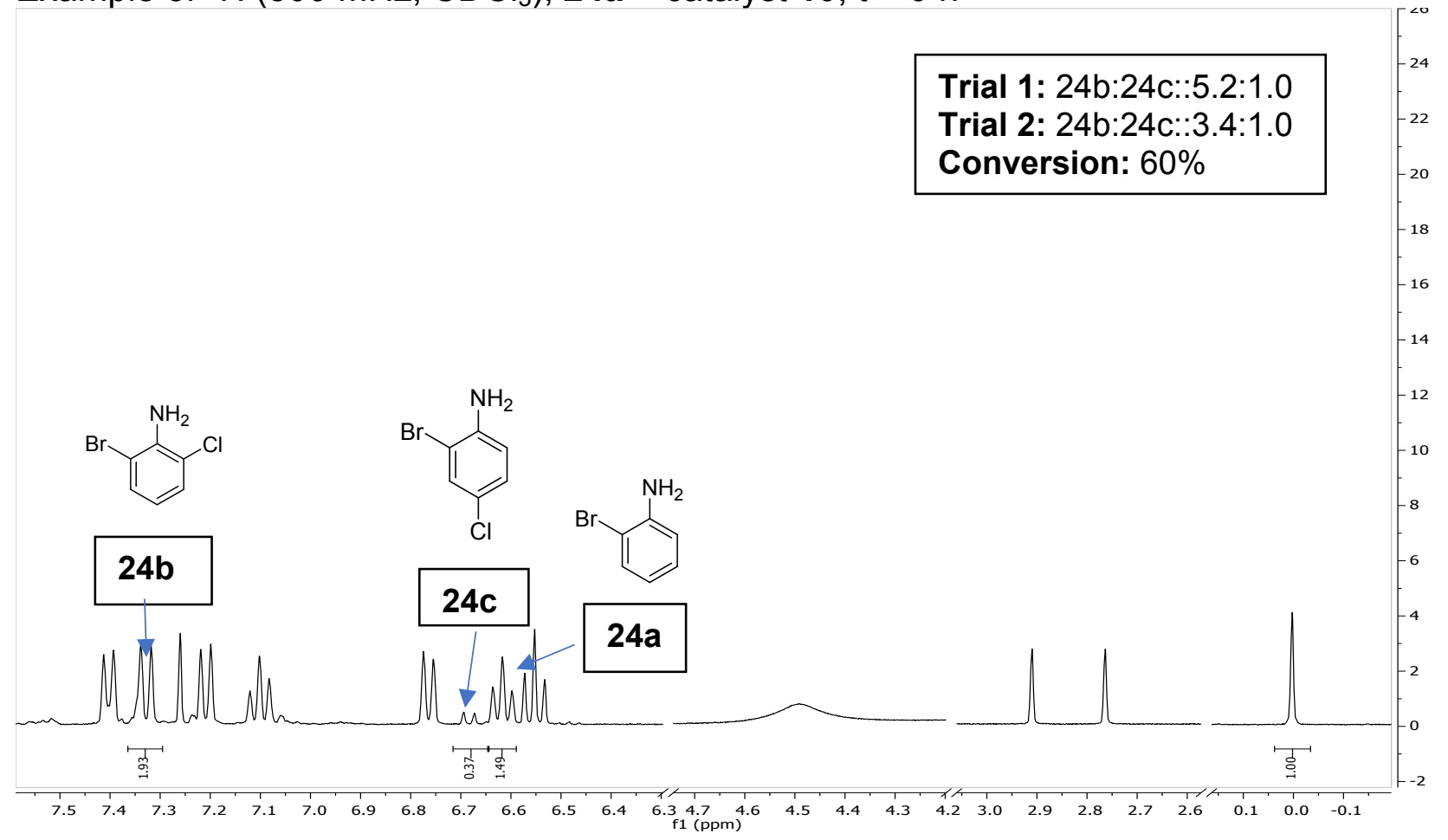

Example of ${ }^{1} \mathrm{H}\left(500 \mathrm{MHz}, \mathrm{CDCl}_{3}\right), 24 \mathrm{a}+$ catalyst $10 ; \mathrm{t}=18 \mathrm{~h}$ 


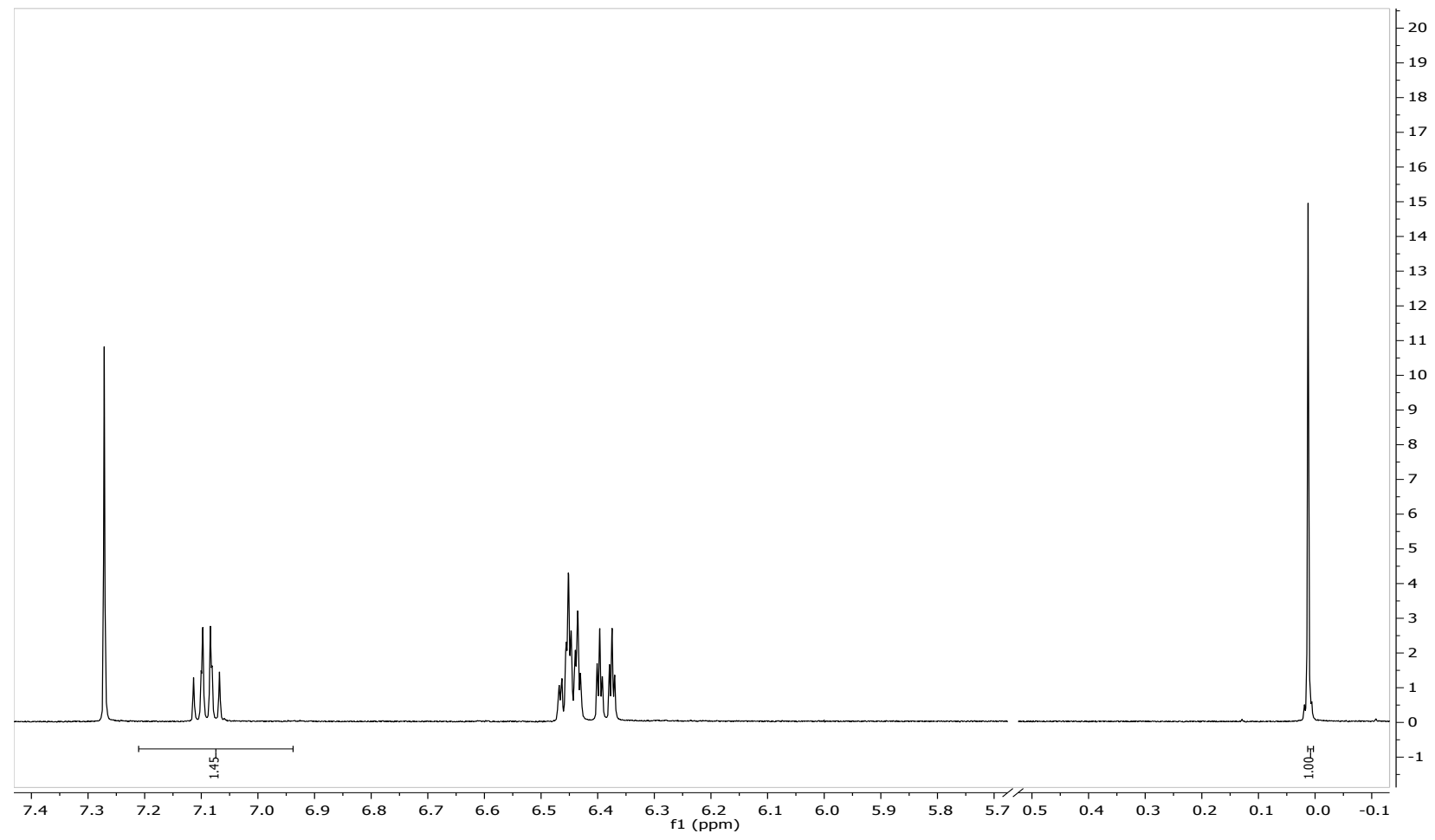

Example of ${ }^{1} \mathrm{H}\left(500 \mathrm{MHz}, \mathrm{CDCl}_{3}\right), 25 \mathrm{a}+$ no catalyst; $\mathrm{t}=0 \mathrm{~h}$

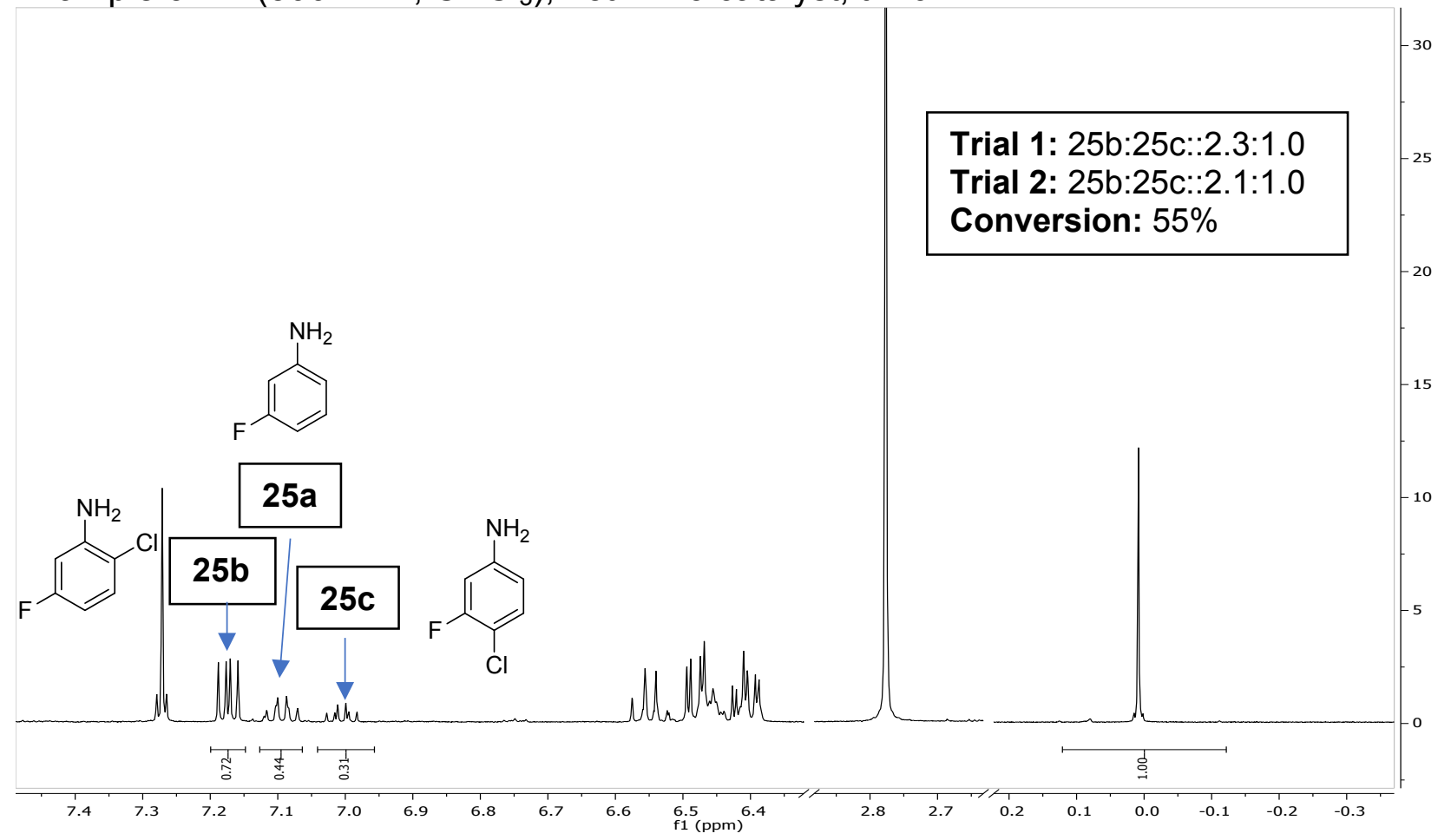

Example of ${ }^{1} \mathrm{H}\left(500 \mathrm{MHz}, \mathrm{CDCl}_{3}\right), 25 \mathrm{a}+$ no catalyst; $\mathrm{t}=12 \mathrm{~h}$ 


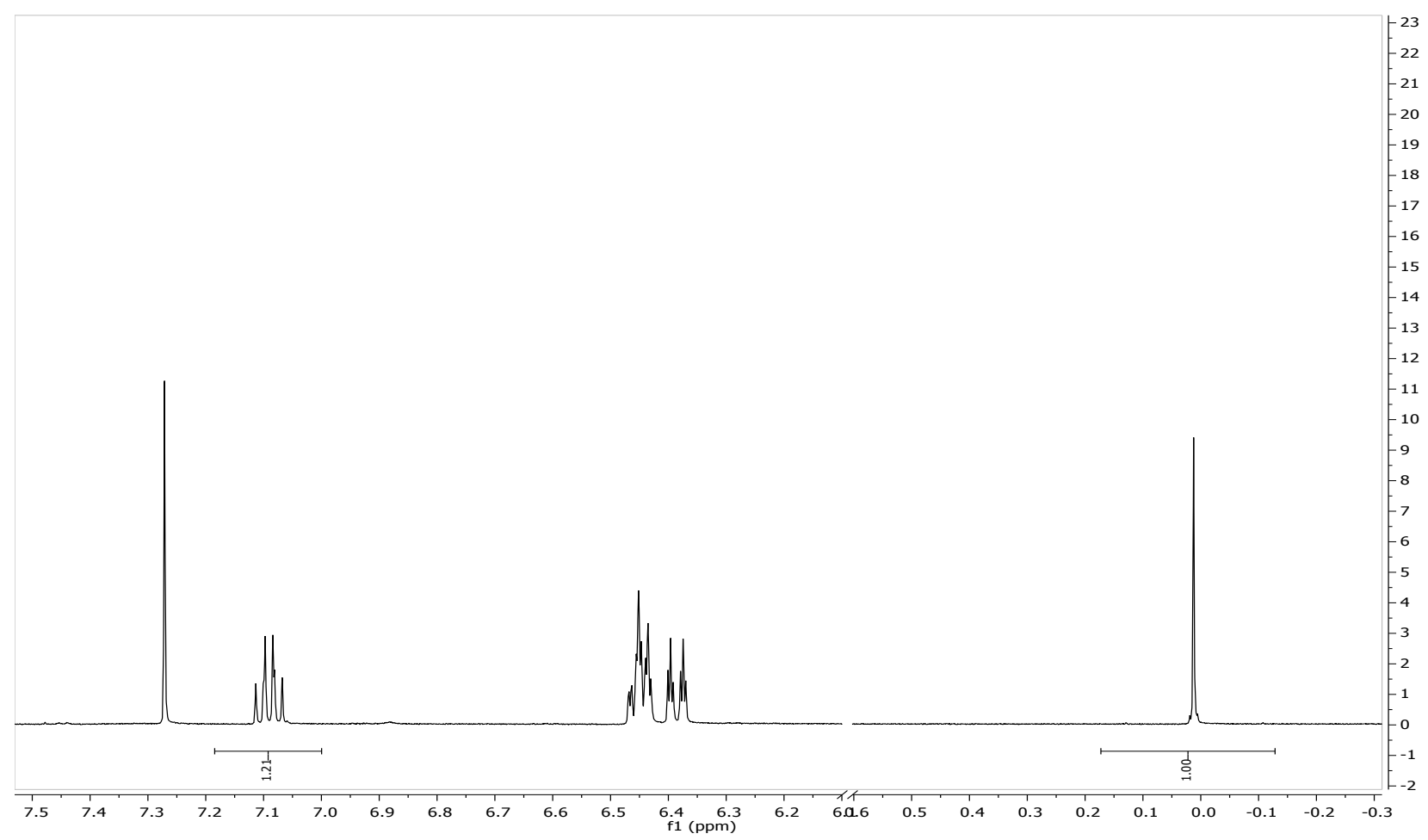

Example of ${ }^{1} \mathrm{H}\left(500 \mathrm{MHz}, \mathrm{CDCl}_{3}\right), 25 \mathrm{a}+$ catalyst $10 ; \mathrm{t}=0 \mathrm{~h}$

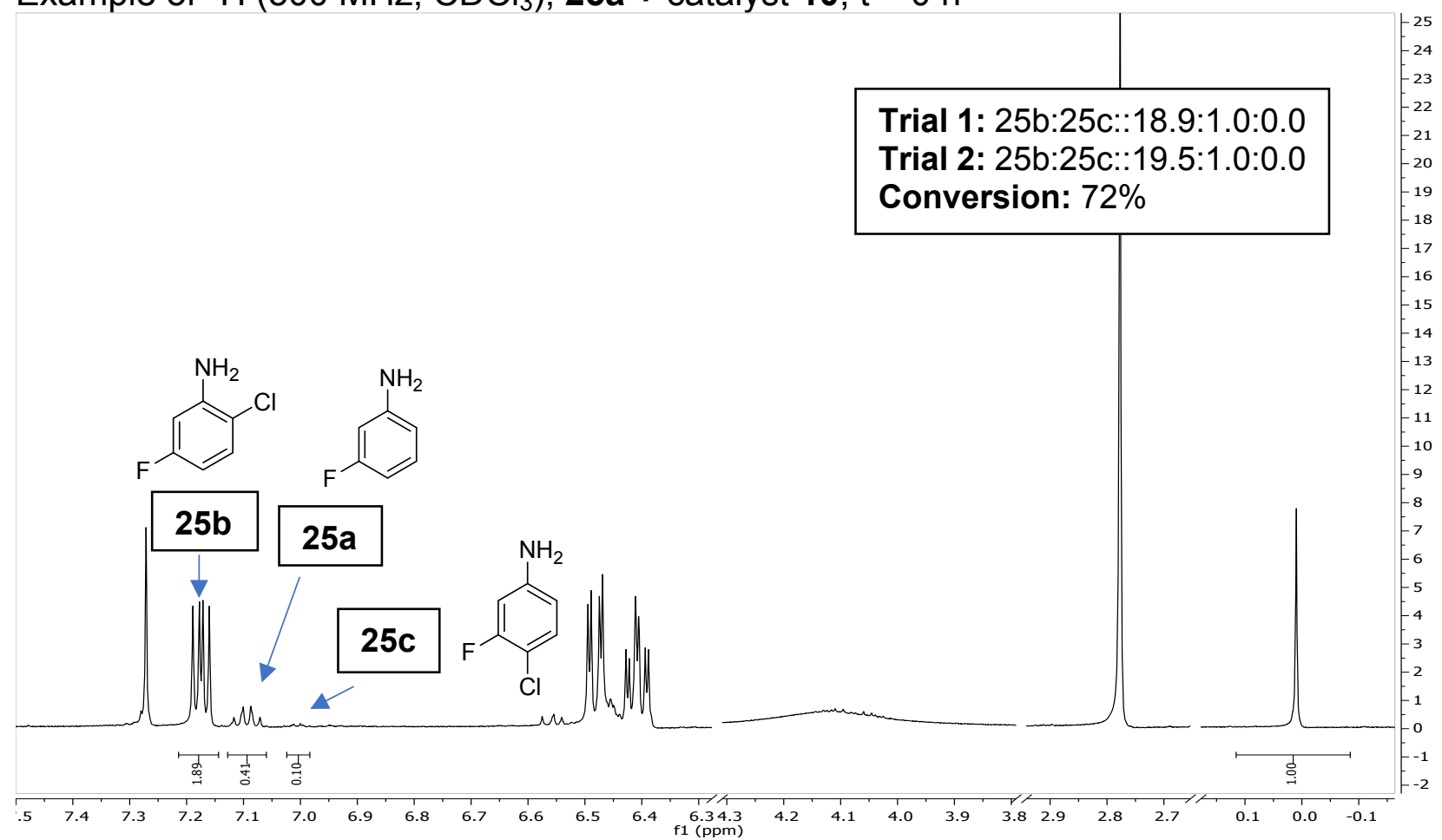

Example of ${ }^{1} \mathrm{H}\left(500 \mathrm{MHz}, \mathrm{CDCl}_{3}\right), 25 \mathrm{a}+$ catalyst $10 ; \mathrm{t}=12 \mathrm{~h}$ 


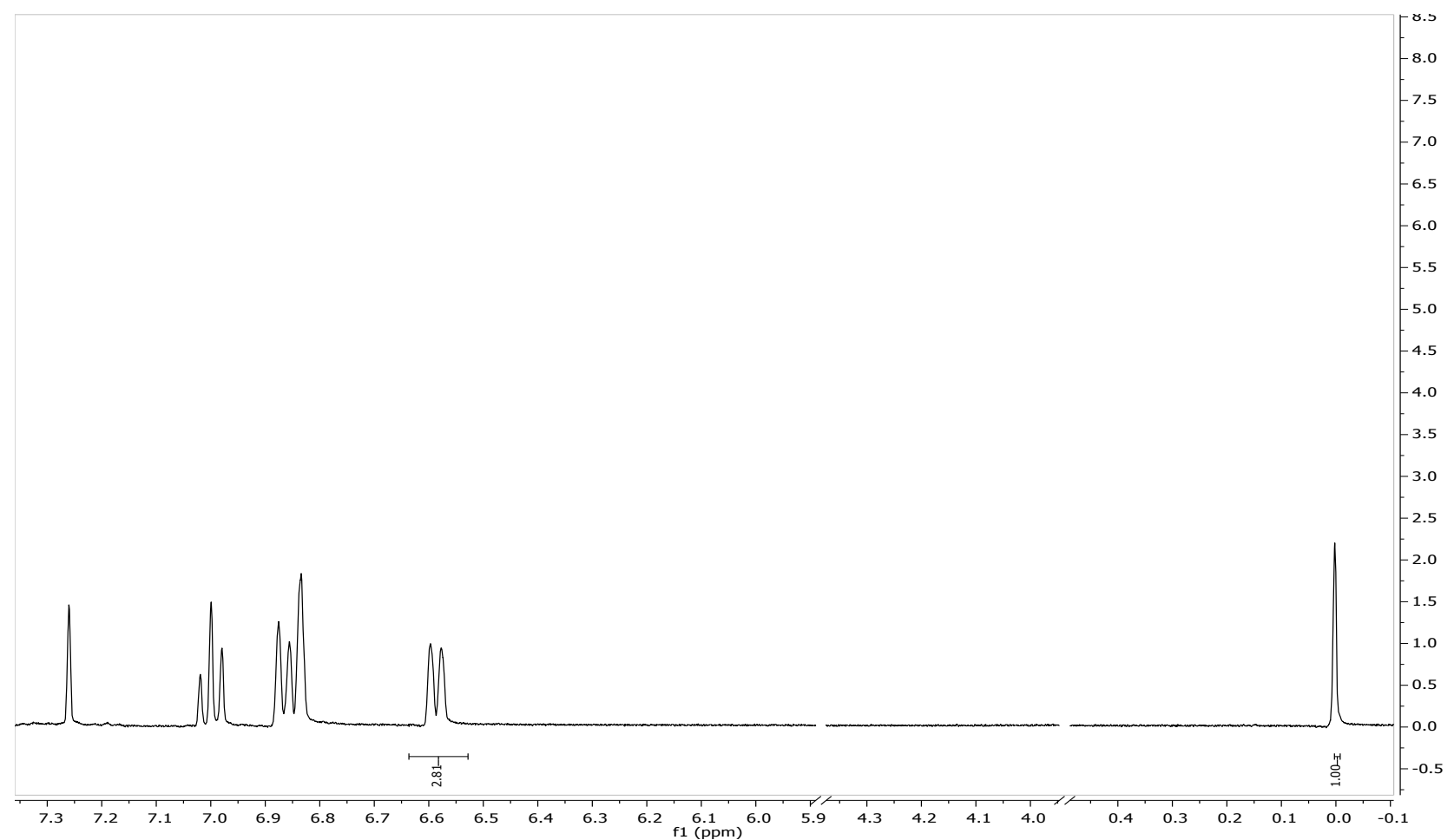

Example of ${ }^{1} \mathrm{H}\left(500 \mathrm{MHz}, \mathrm{CDCl}_{3}\right), \mathbf{2 6 a}+$ No catalyst; $\mathrm{t}=0 \mathrm{~h}$

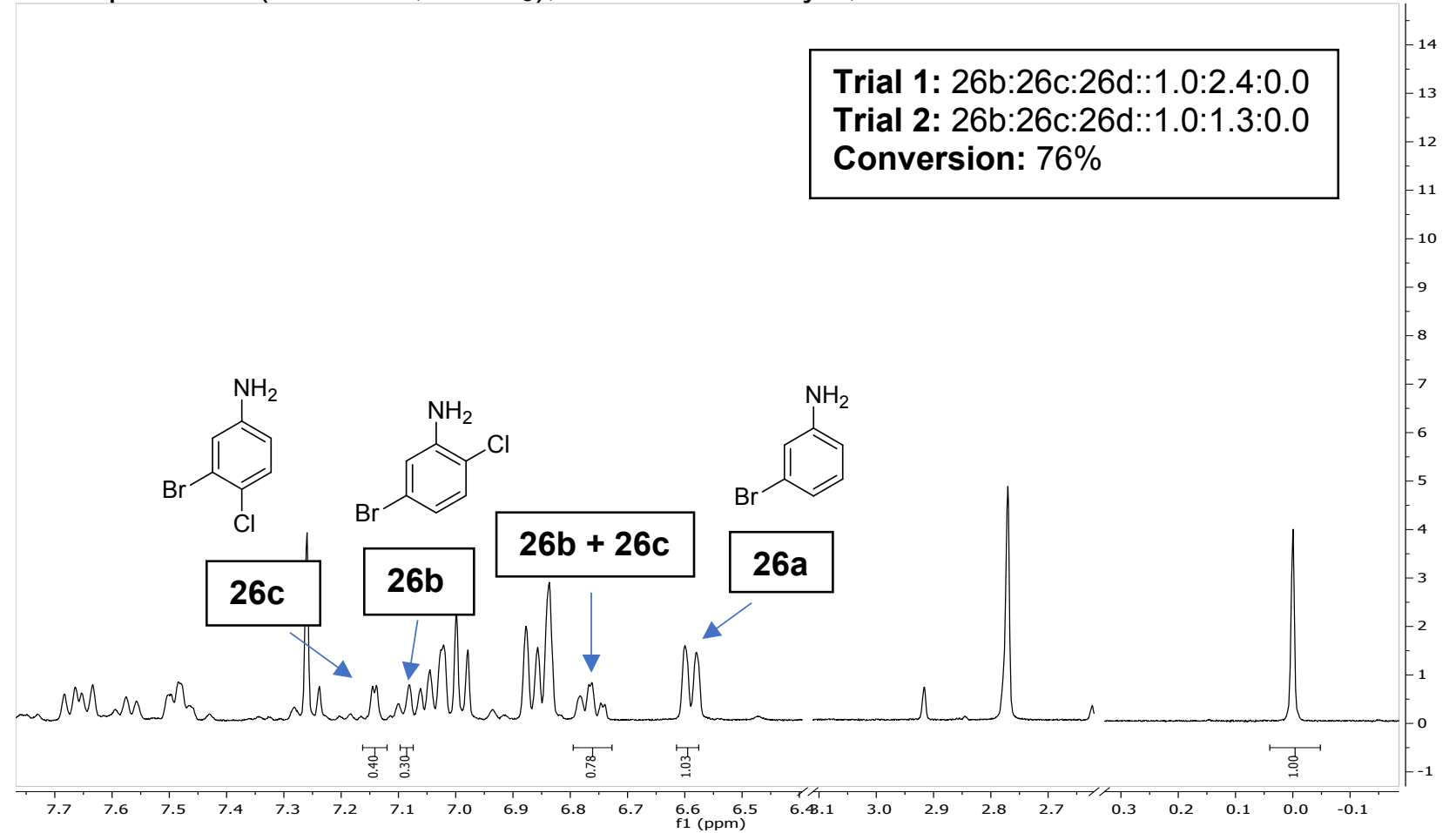

Example of ${ }^{1} \mathrm{H}\left(500 \mathrm{MHz}, \mathrm{CDCl}_{3}\right), 26 \mathrm{a}+$ No catalyst; $\mathrm{t}=18 \mathrm{~h}$ 


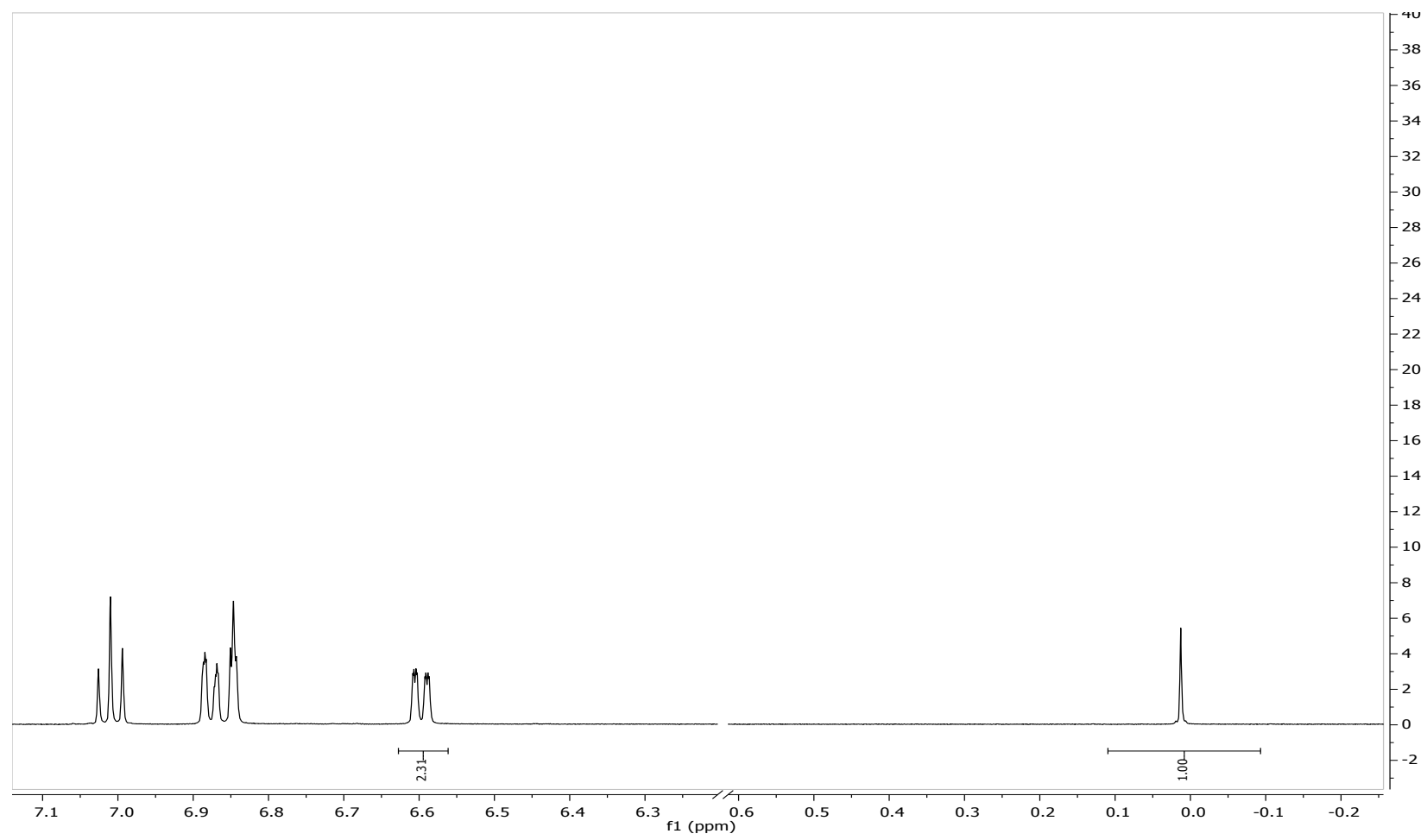

Example of ${ }^{1} \mathrm{H}\left(500 \mathrm{MHz}, \mathrm{CDCl}_{3}\right), \mathbf{2 6 a}+$ catalyst $10 ; \mathrm{t}=0 \mathrm{~h}$

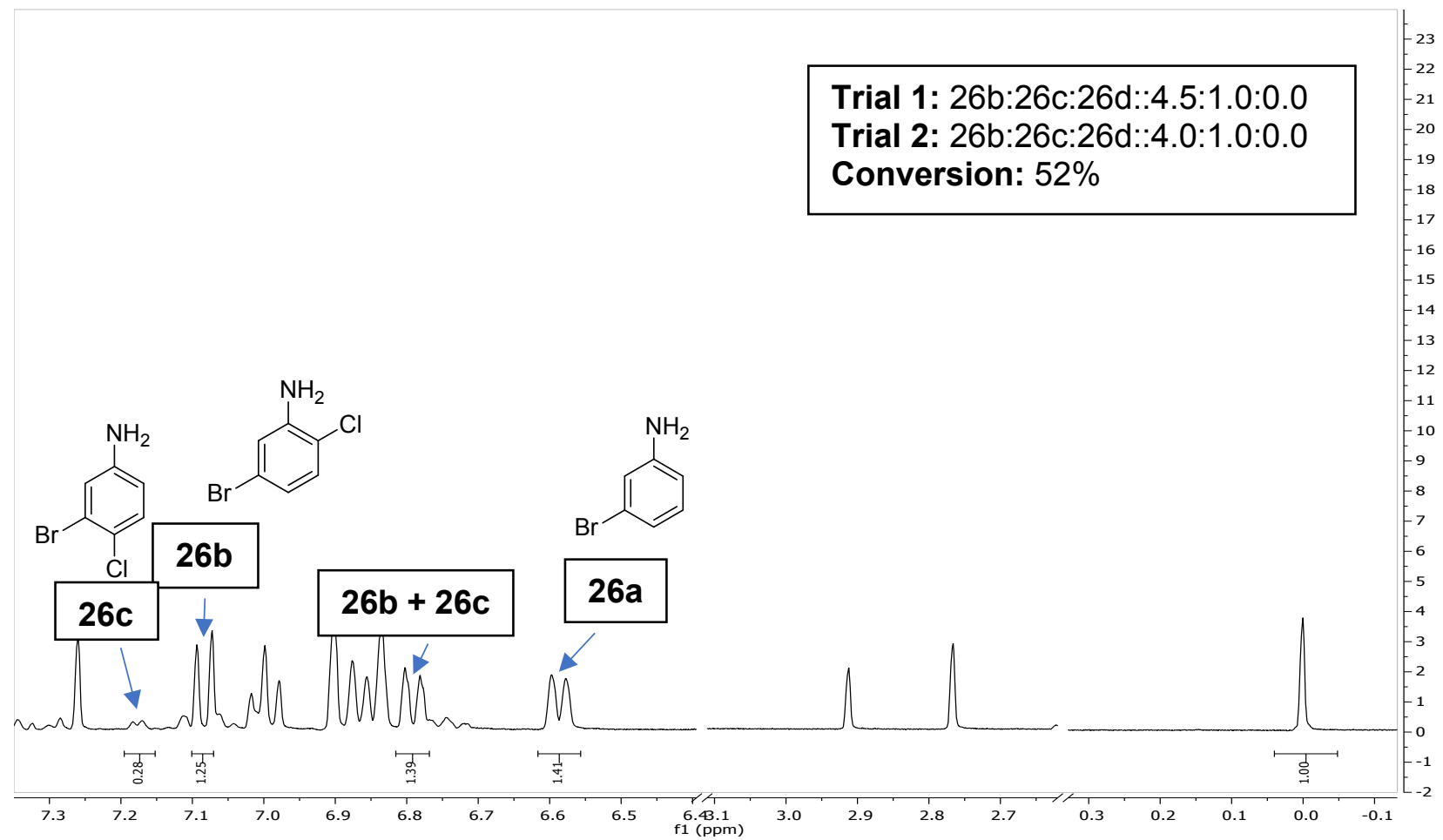

Example of ${ }^{1} \mathrm{H}\left(500 \mathrm{MHz}, \mathrm{CDCl}_{3}\right), 26 \mathrm{a}+$ catalyst $10 ; \mathrm{t}=18 \mathrm{~h}$ 


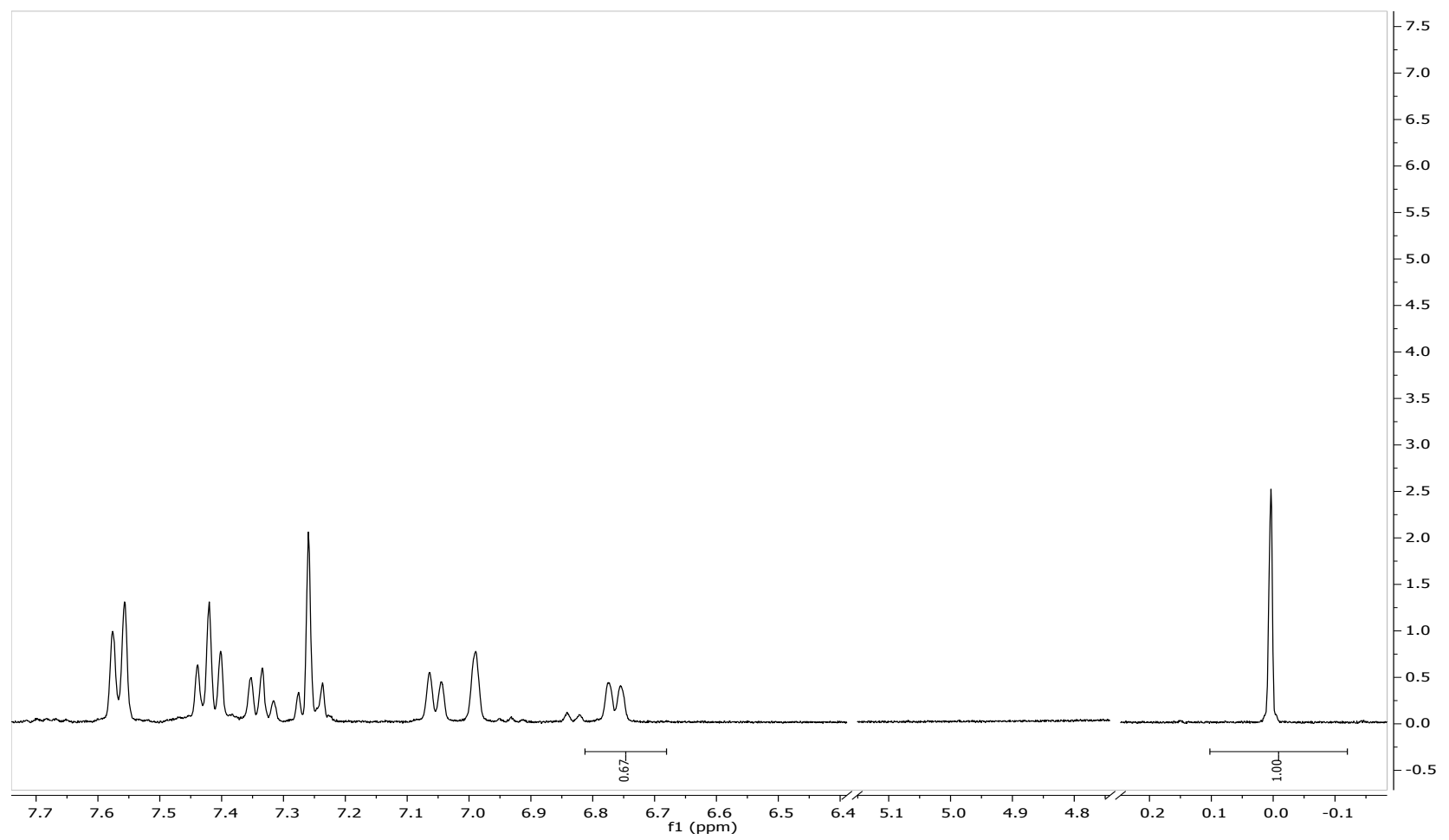

Example of ${ }^{1} \mathrm{H}\left(500 \mathrm{MHz}, \mathrm{CDCl}_{3}\right), 27 \mathrm{a}+$ no catalyst; $\mathrm{t}=0 \mathrm{~h}$

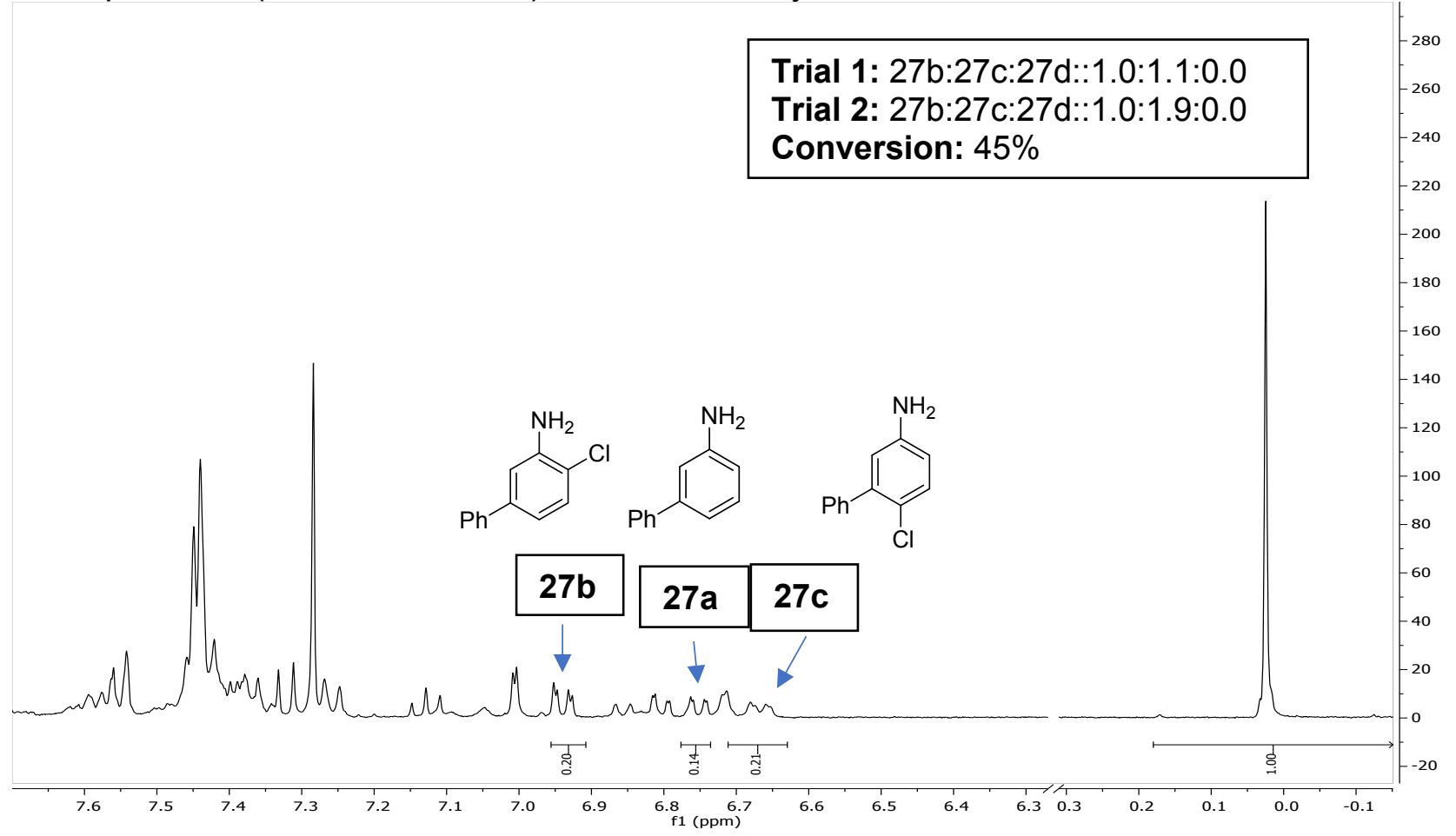

Example of ${ }^{1} \mathrm{H}\left(400 \mathrm{MHz}, \mathrm{CDCl}_{3}\right), 27 \mathrm{a}+$ no catalyst; $\mathrm{t}=12 \mathrm{~h}$ 


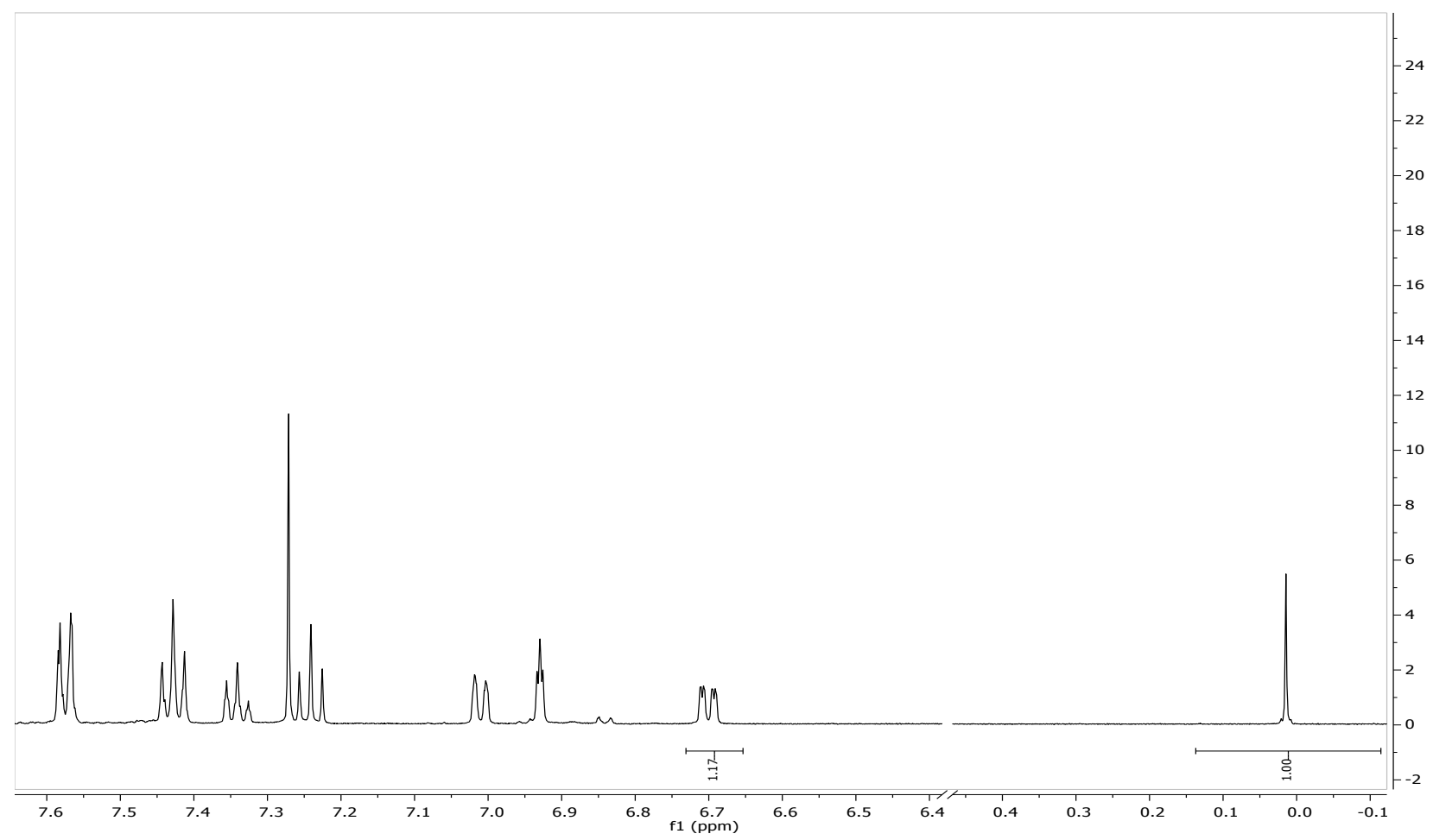

Example of ${ }^{1} \mathrm{H}\left(500 \mathrm{MHz}, \mathrm{CDCl}_{3}\right), 27 \mathrm{a}+$ catalyst $10 ; \mathrm{t}=0 \mathrm{~h}$

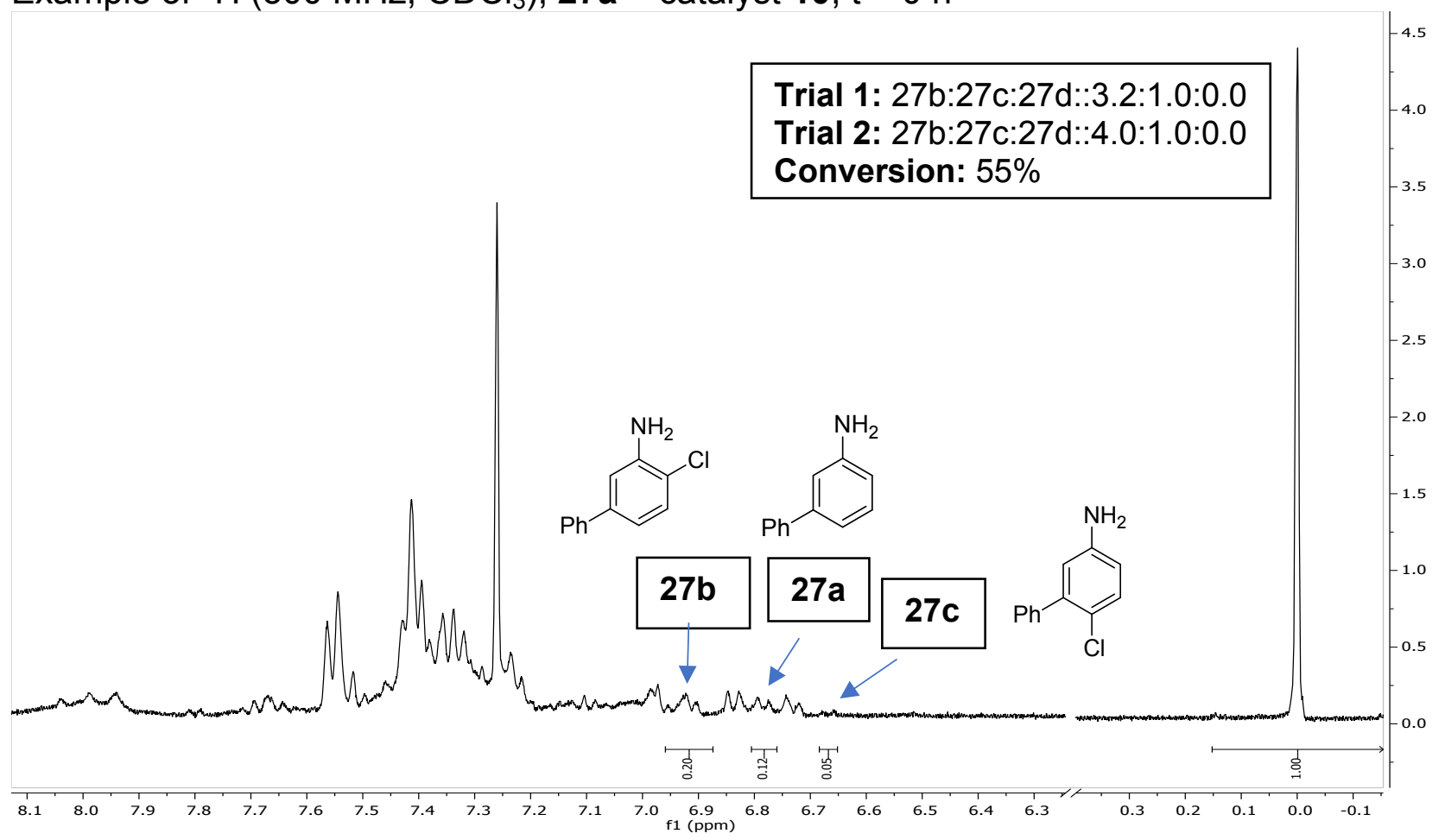

Example of ${ }^{1} \mathrm{H}\left(500 \mathrm{MHz}, \mathrm{CDCl}_{3}\right), 27 \mathrm{a}+$ catalyst $10 ; \mathrm{t}=12 \mathrm{~h}$ 


\section{B. NMR Spectra of Purified Products}

${ }^{1} \mathrm{H}(500 \mathrm{MHz}){ }^{13} \mathrm{C}(126 \mathrm{MHz})$ and ${ }^{19} \mathrm{~F}(376 \mathrm{MHz})$ NMR Spectra of $\mathbf{N}-((3 \mathrm{aS}, 7 \mathrm{aS})$-hexahydro-1Hbenzo[d]imidazol-2(3H)-ylidene)-3,5-bis(trifluoromethyl)aniline (1a)

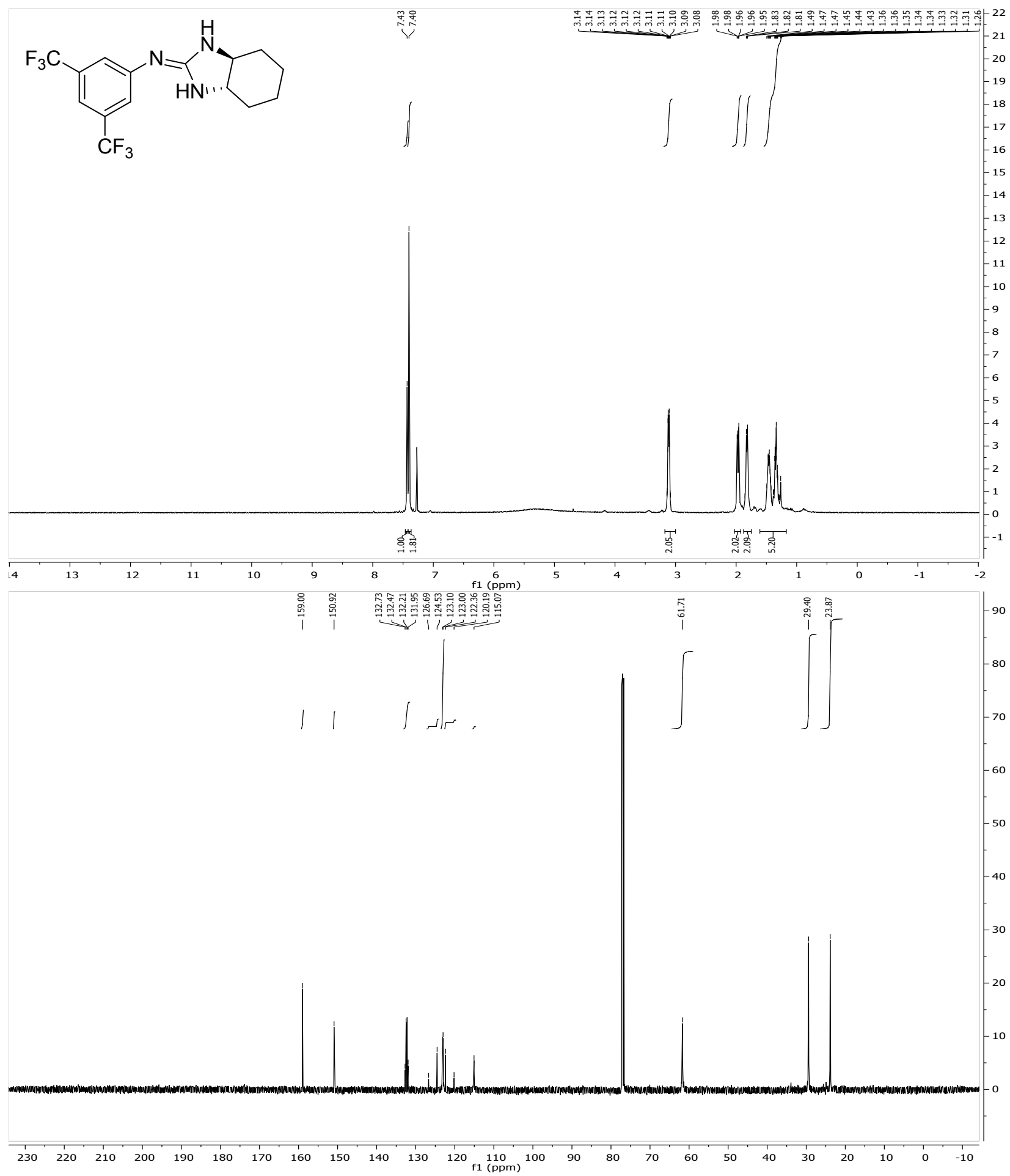




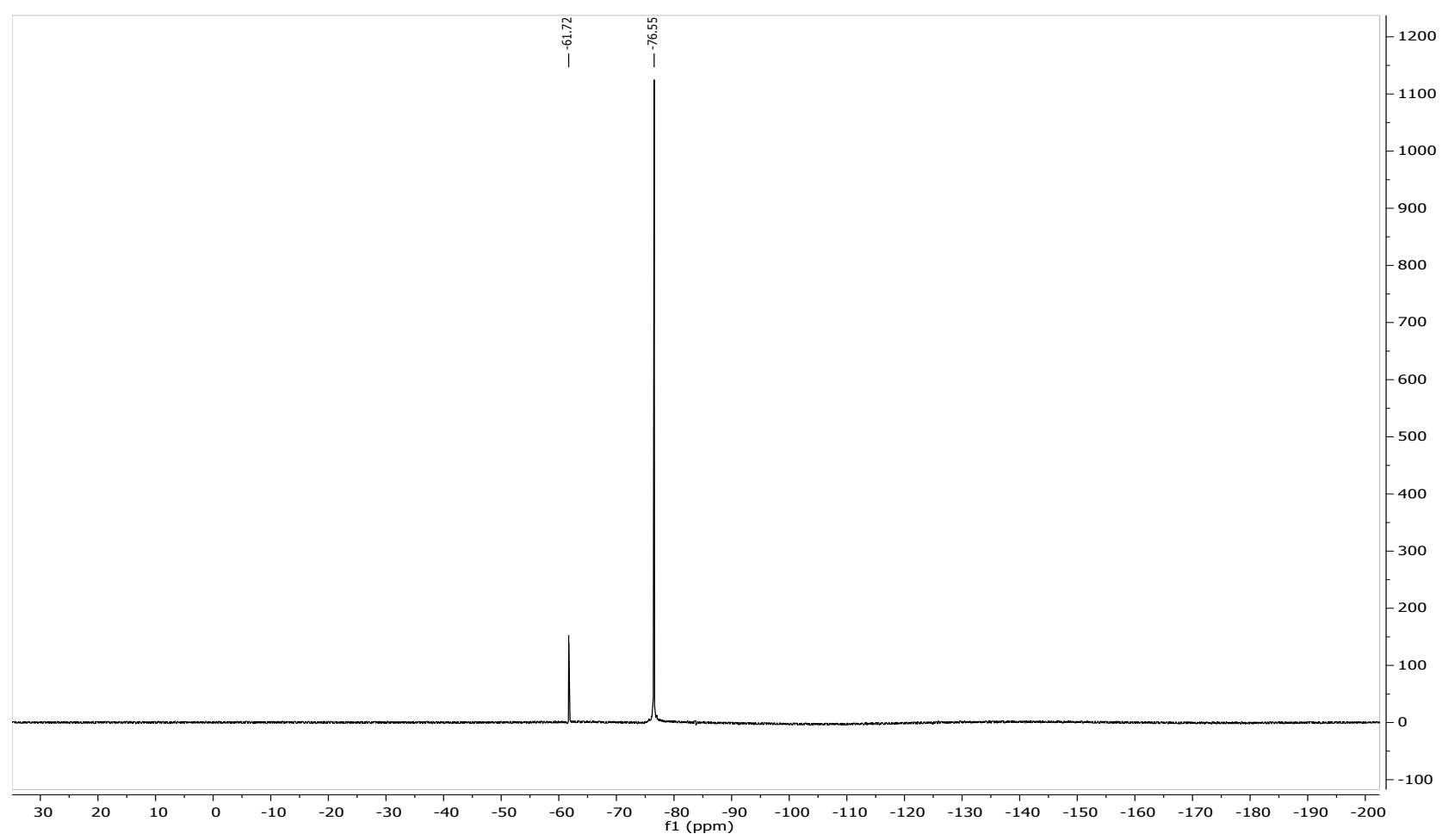

S-38 
${ }^{1} \mathrm{H}(500 \mathrm{MHz})$ and ${ }^{13} \mathrm{C}(126 \mathrm{MHz})$ NMR Spectra $\left(\mathrm{CDCl}_{3}\right)$ of 1,2-dimesityldiselane

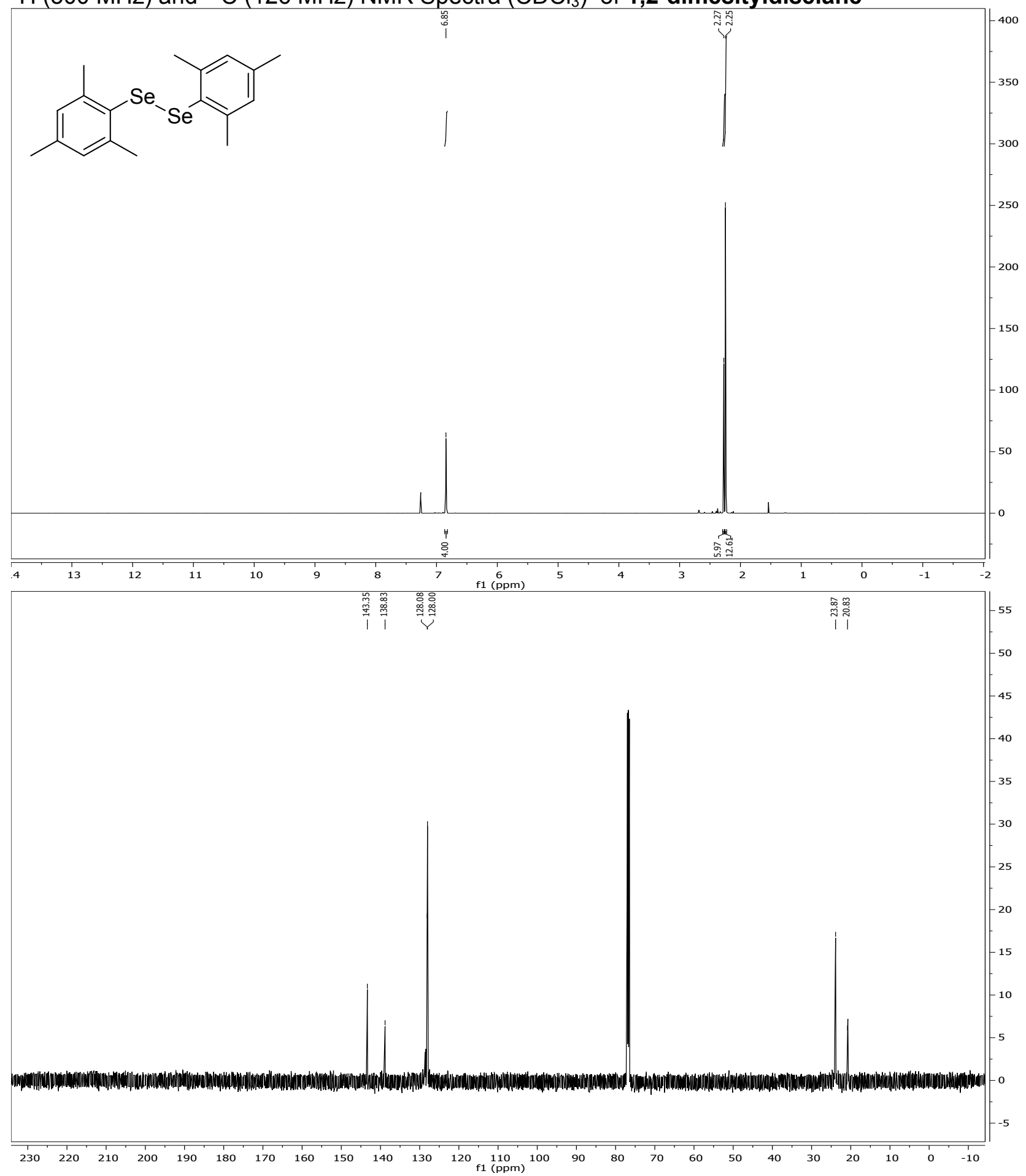


${ }^{1} \mathrm{H}(500 \mathrm{MHz})$ and ${ }^{13} \mathrm{C}(126 \mathrm{MHz}) \mathrm{NMR}$ Spectra $\left(\mathrm{CDCl}_{3}\right)$ of tert-butyl ((1S,2S)-2-(mesitylthio)2,3-dihydro-1H-inden-1-yl)carbamate

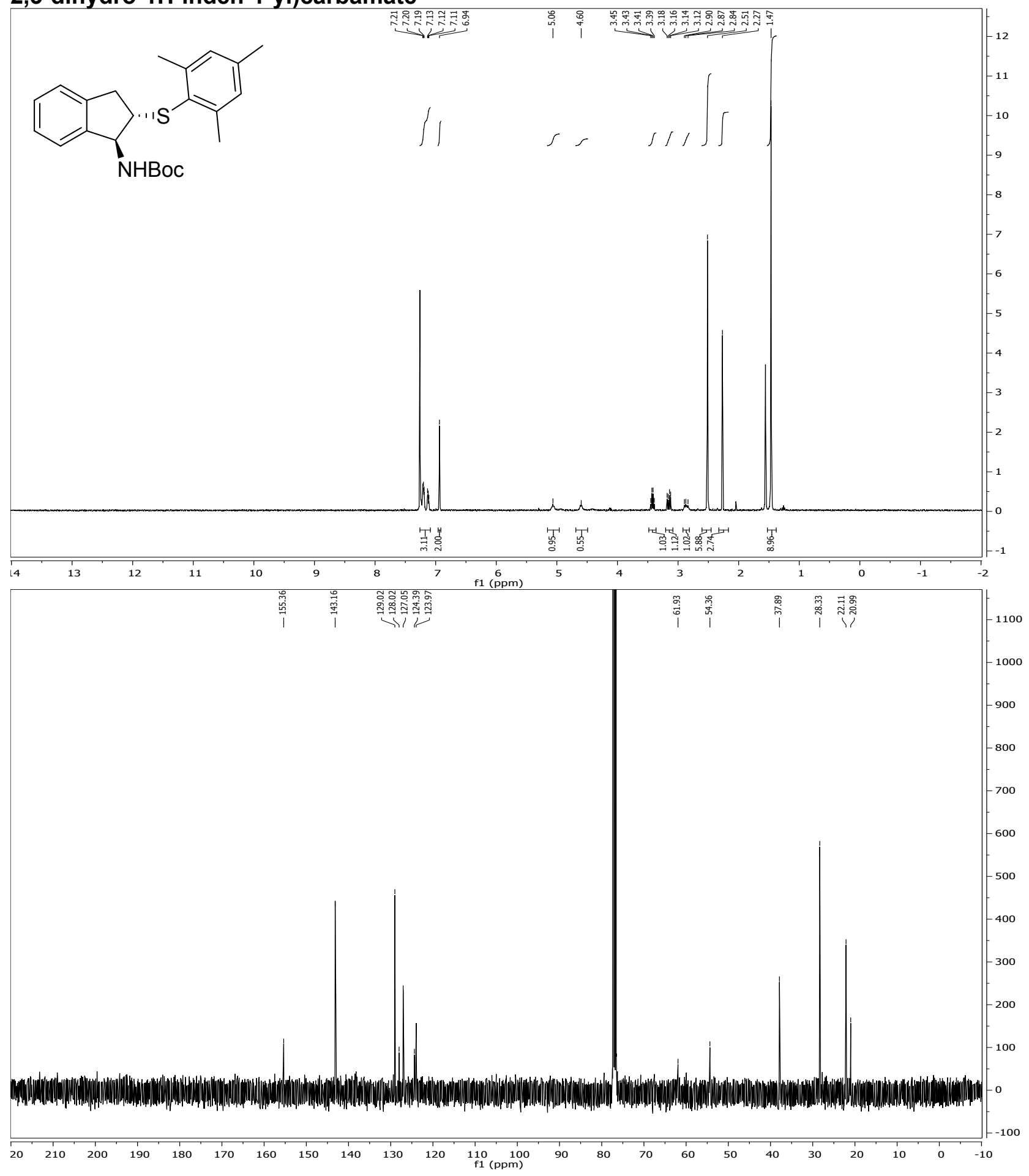


${ }^{1} \mathrm{H}(500 \mathrm{MHz})$ and ${ }^{13} \mathrm{C}(126 \mathrm{MHz})$ NMR Spectra $\left(\mathrm{CDCl}_{3}\right)$ of tert-butyl ((1S,2S)-2-(mesitylselanyl)2,3-dihydro-1H-inden-1-yl)carbamate

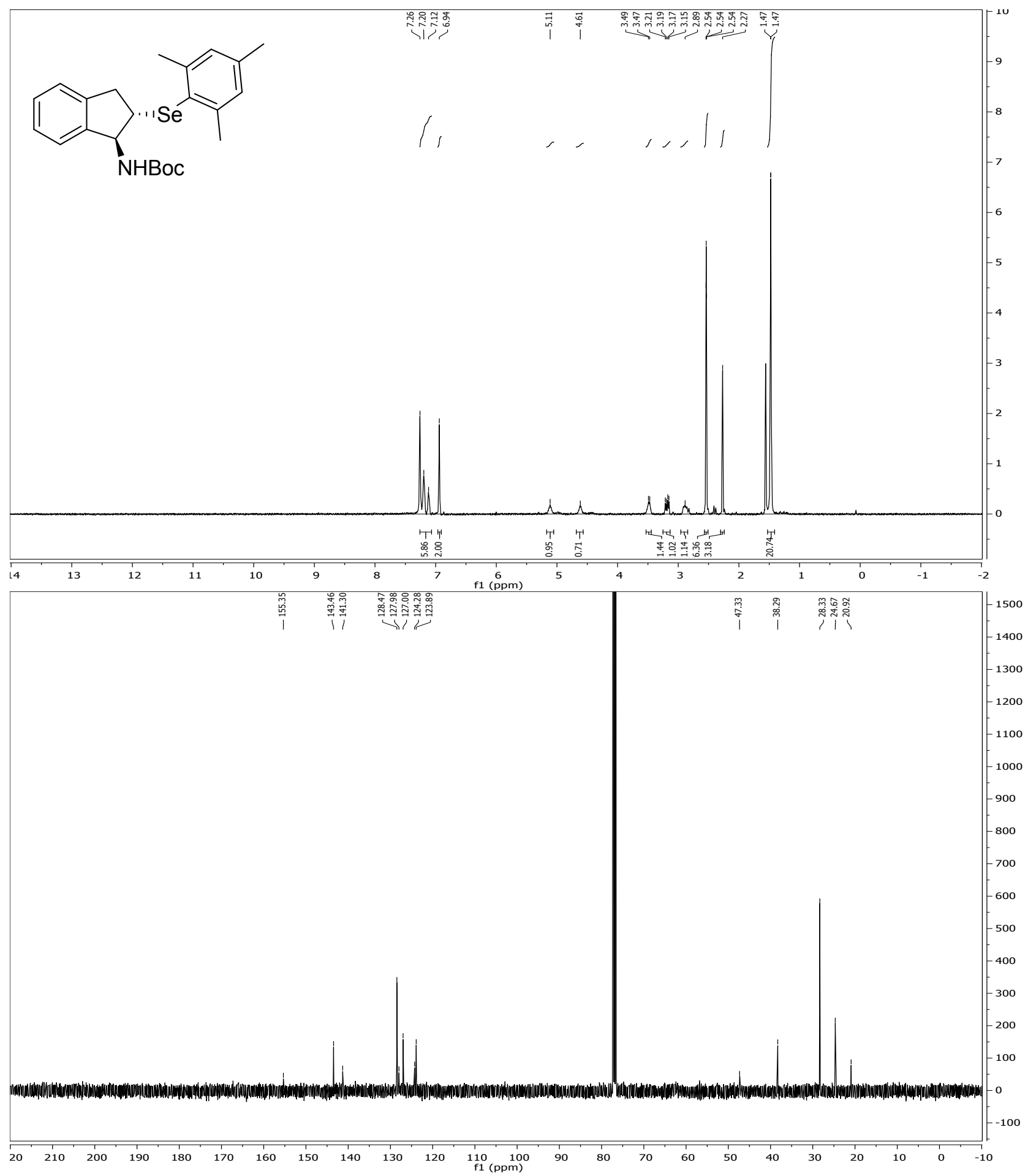


${ }^{1} \mathrm{H}(400 \mathrm{MHz})$ and ${ }^{13} \mathrm{C}(126 \mathrm{MHz}) \mathrm{NMR}$ Spectra $\left(\mathrm{CDCl}_{3}\right)$ of (1S,2S)-2-(mesitylthio)-2,3-dihydro1H-inden-1-amine

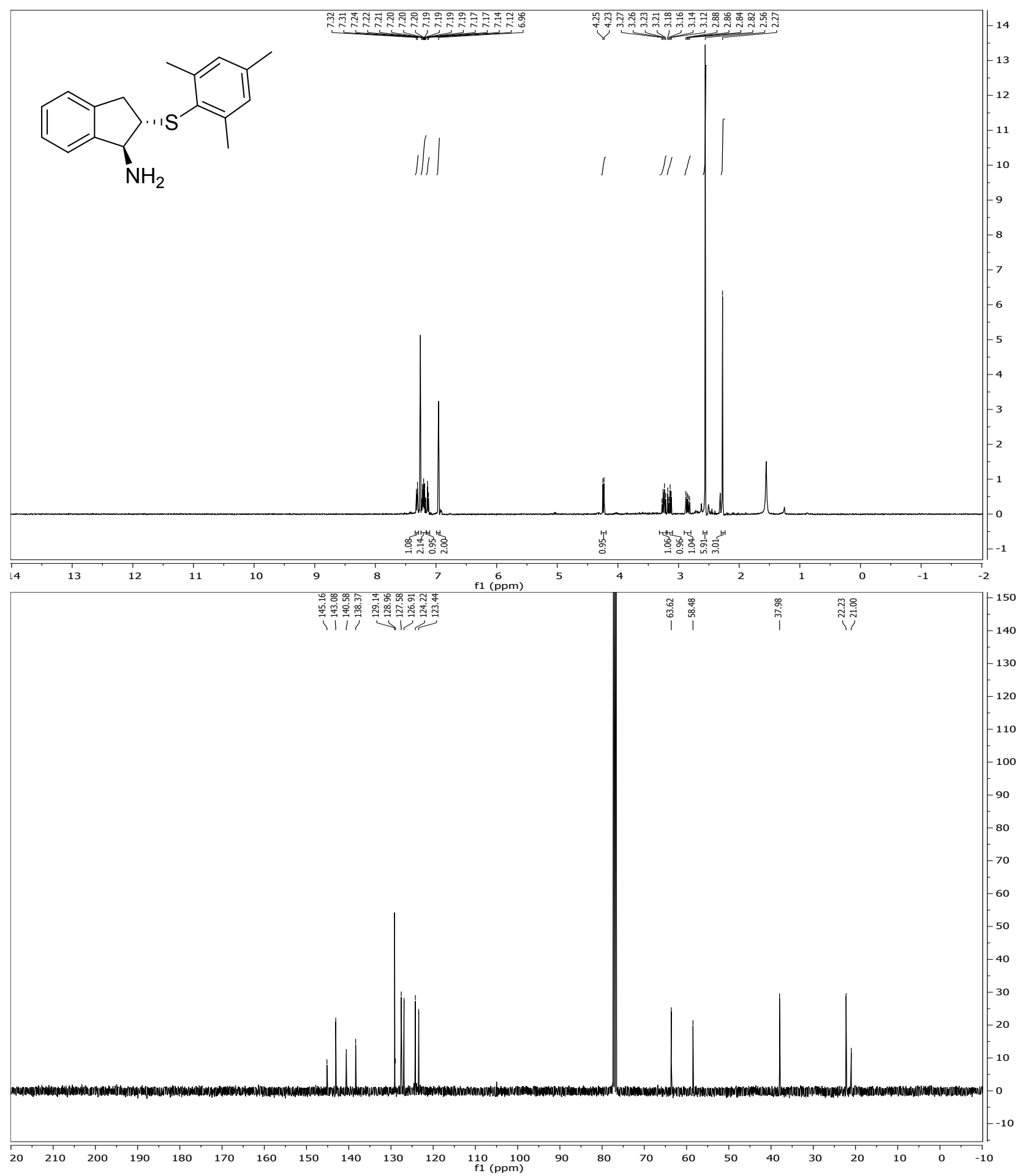


${ }^{1} \mathrm{H}(500 \mathrm{MHz})$ and ${ }^{13} \mathrm{C}(126 \mathrm{MHz}) \mathrm{NMR}$ Spectra $\left(\mathrm{CDCl}_{3}\right)$ of $(\mathbf{1 S}, 2 \mathrm{~S})-2-($ mesitylselanyl)-2,3dihydro-1H-inden-1-amine

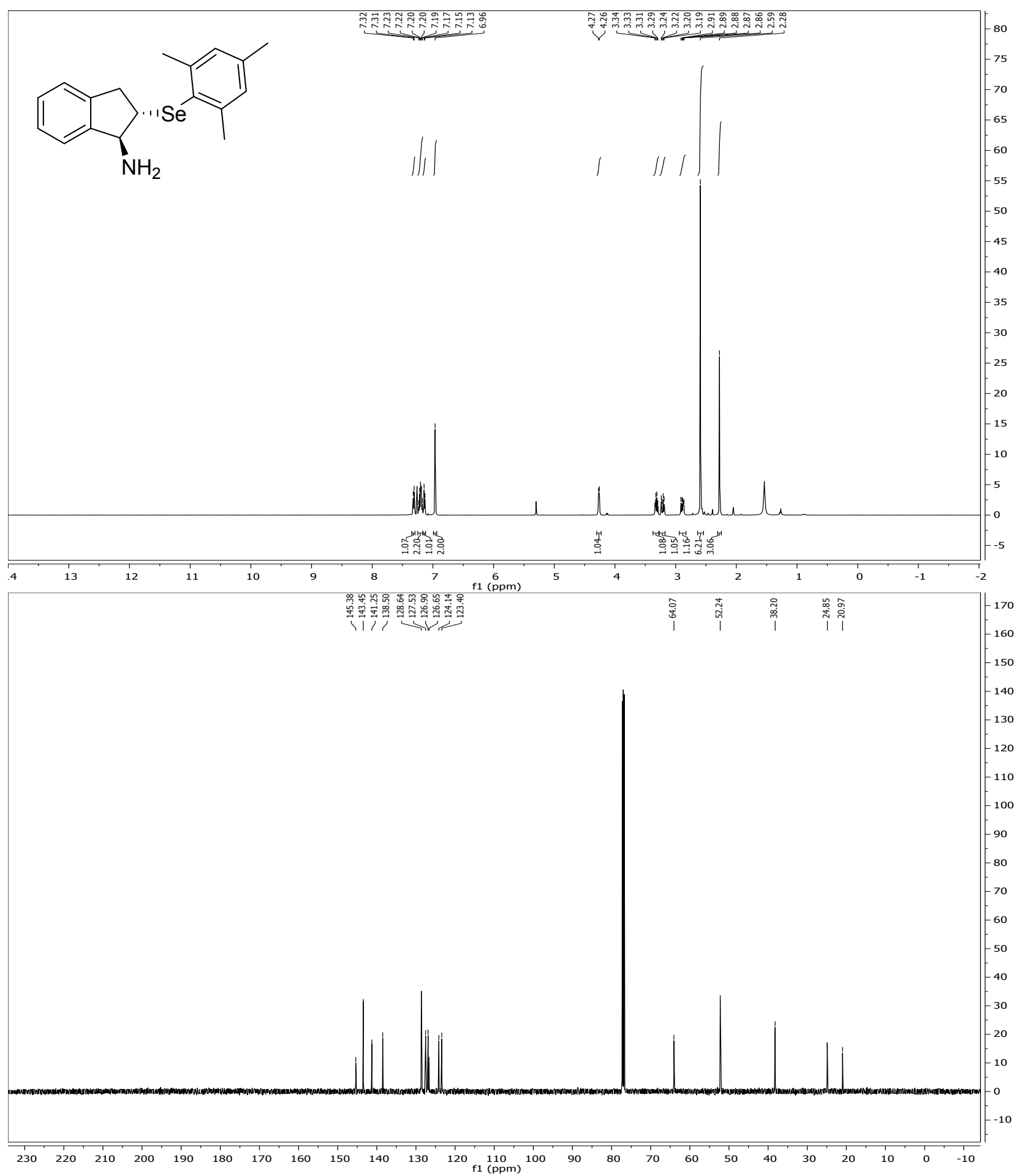


${ }^{1} \mathrm{H} \quad\left(400 \mathrm{MHz}, \mathrm{DMSO}-d_{6}\right)$ and ${ }^{13} \mathrm{C} \quad\left(126 \mathrm{MHz}, \mathrm{CDCl}_{3}\right)$ NMR Spectra of 1-(3,5bis(trifluoromethyl)phenyl)-3-((1S,2S)-2-(phenylthio)-2,3-dihydro-1H-inden-1-yl)thiourea (7)
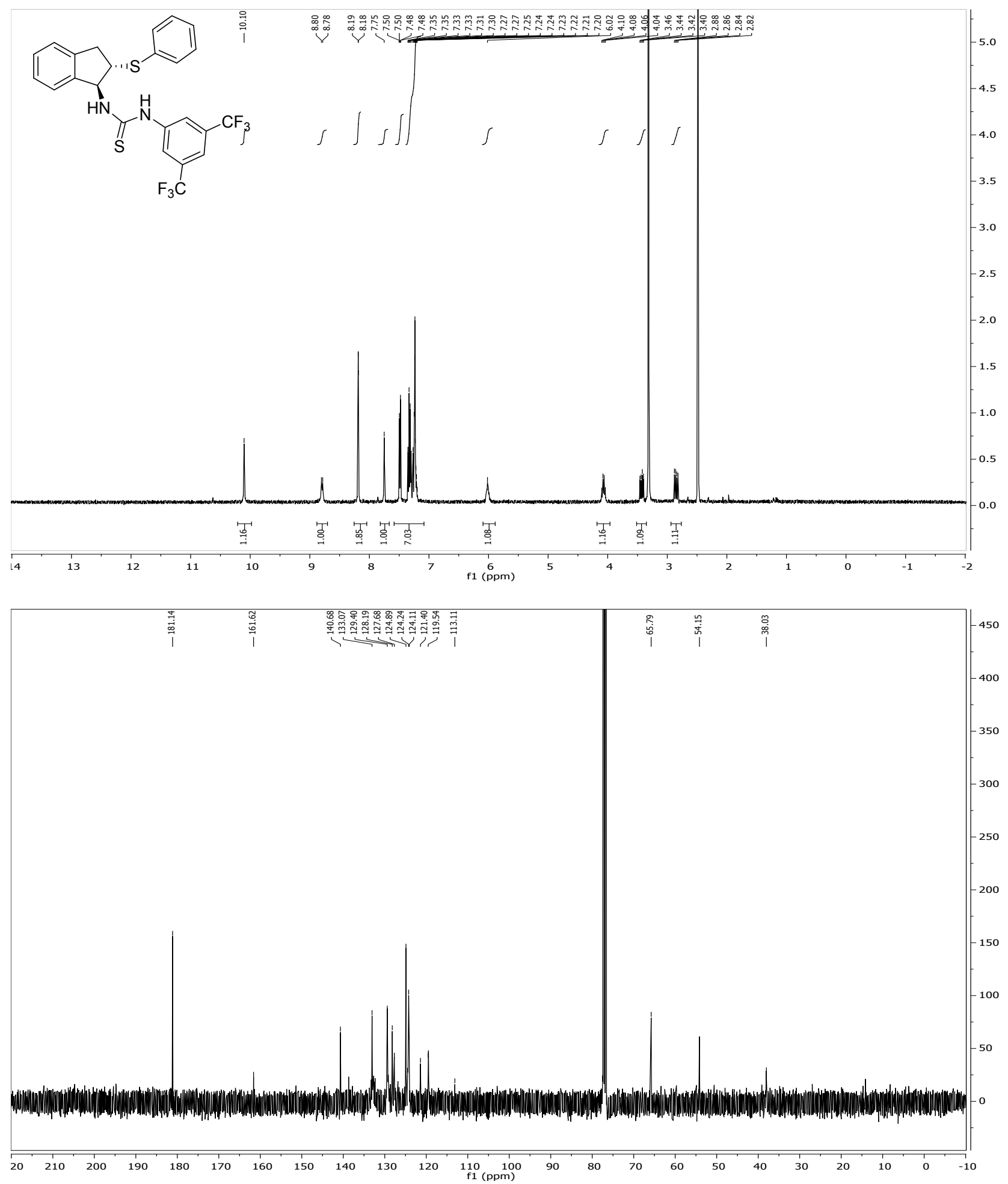
${ }^{1} \mathrm{H} \quad\left(400 \mathrm{MHz}, \quad \mathrm{DMSO}-d_{6}\right)$ and ${ }^{13} \mathrm{C} \quad\left(126 \mathrm{MHz}, \mathrm{CDCl}_{3}\right)$ NMR Spectra of 1-(3,5bis(trifluoromethyl)phenyl)-3-((1S,2S)-2-((4-methoxyphenyl)thio)-2,3-dihydro-1H-inden-1yl)thiourea (8)
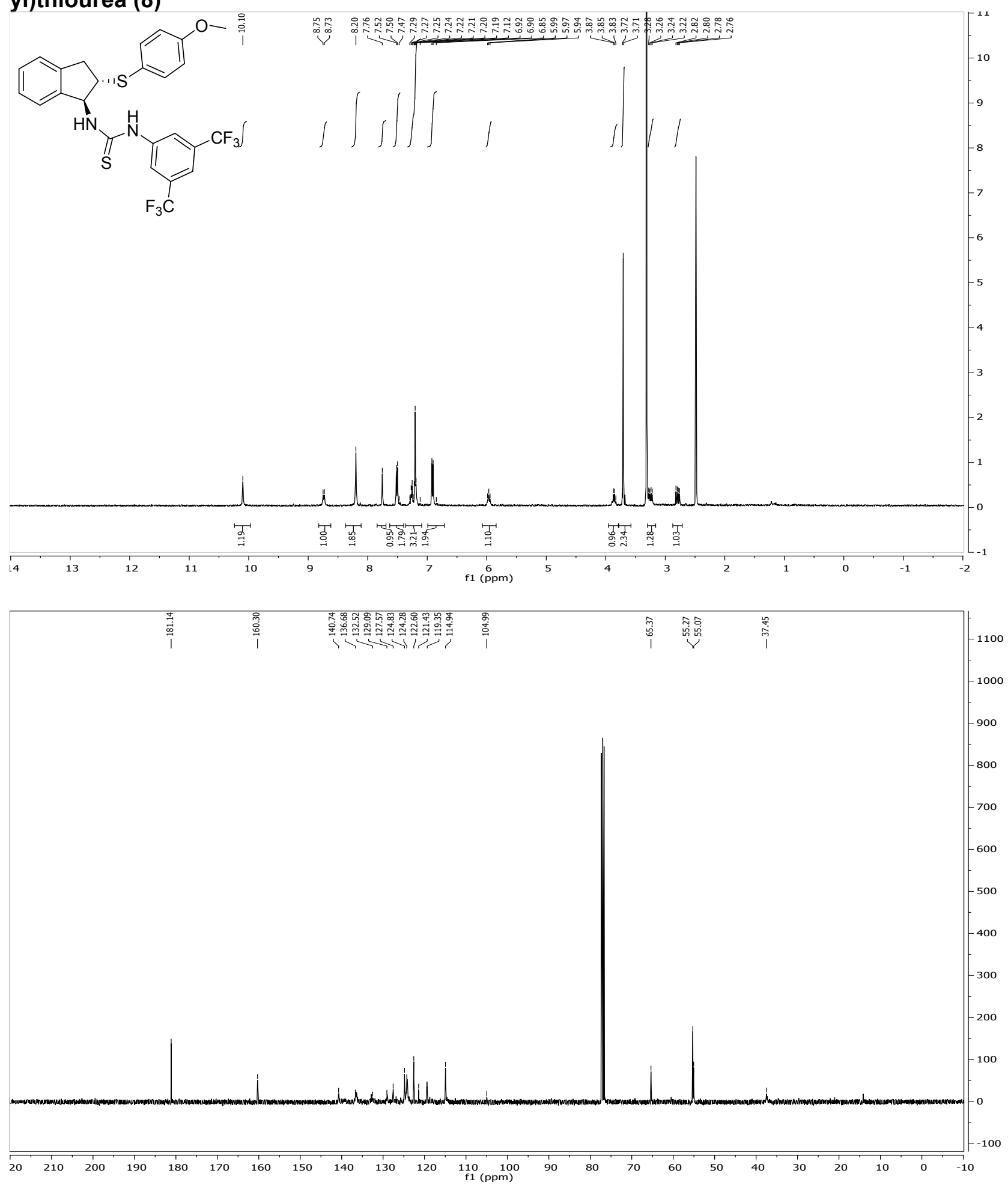
${ }^{1} \mathrm{H}\left(400 \mathrm{MHz}, \mathrm{DMSO}-\mathrm{d}_{6}\right),{ }^{13} \mathrm{C}\left(126 \mathrm{MHz} \mathrm{CDCl}_{3}\right)$ and ${ }^{19} \mathrm{~F}\left(470 \mathrm{MHz}, \mathrm{CDCl}_{3}\right) \mathrm{NMR}$ Spectra of 1(3,5-bis(trifluoromethyl)phenyl)-3-((1S,2S)-2-(mesitylthio)-2,3-dihydro-1H-inden-1yl)thiourea (9)

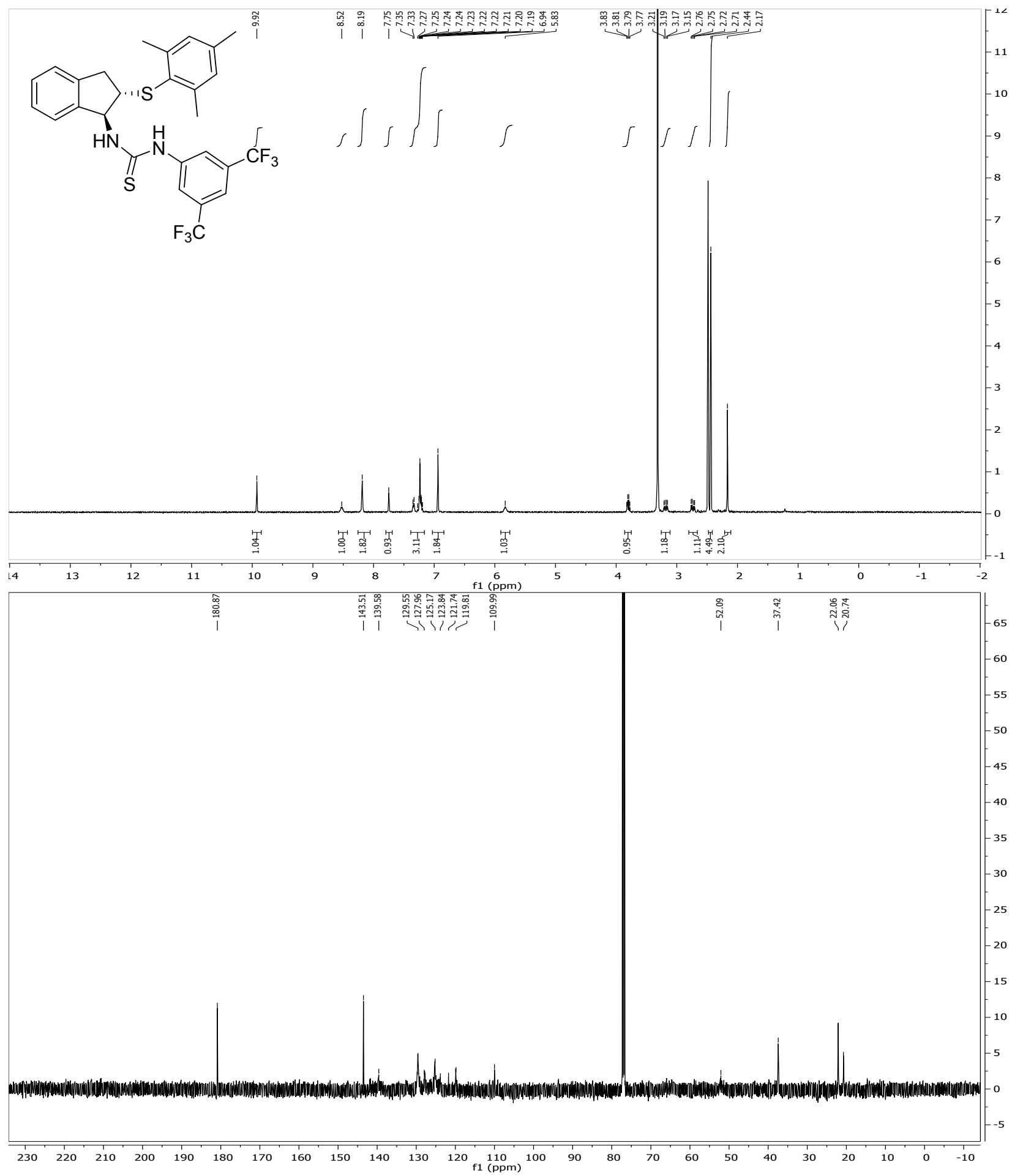




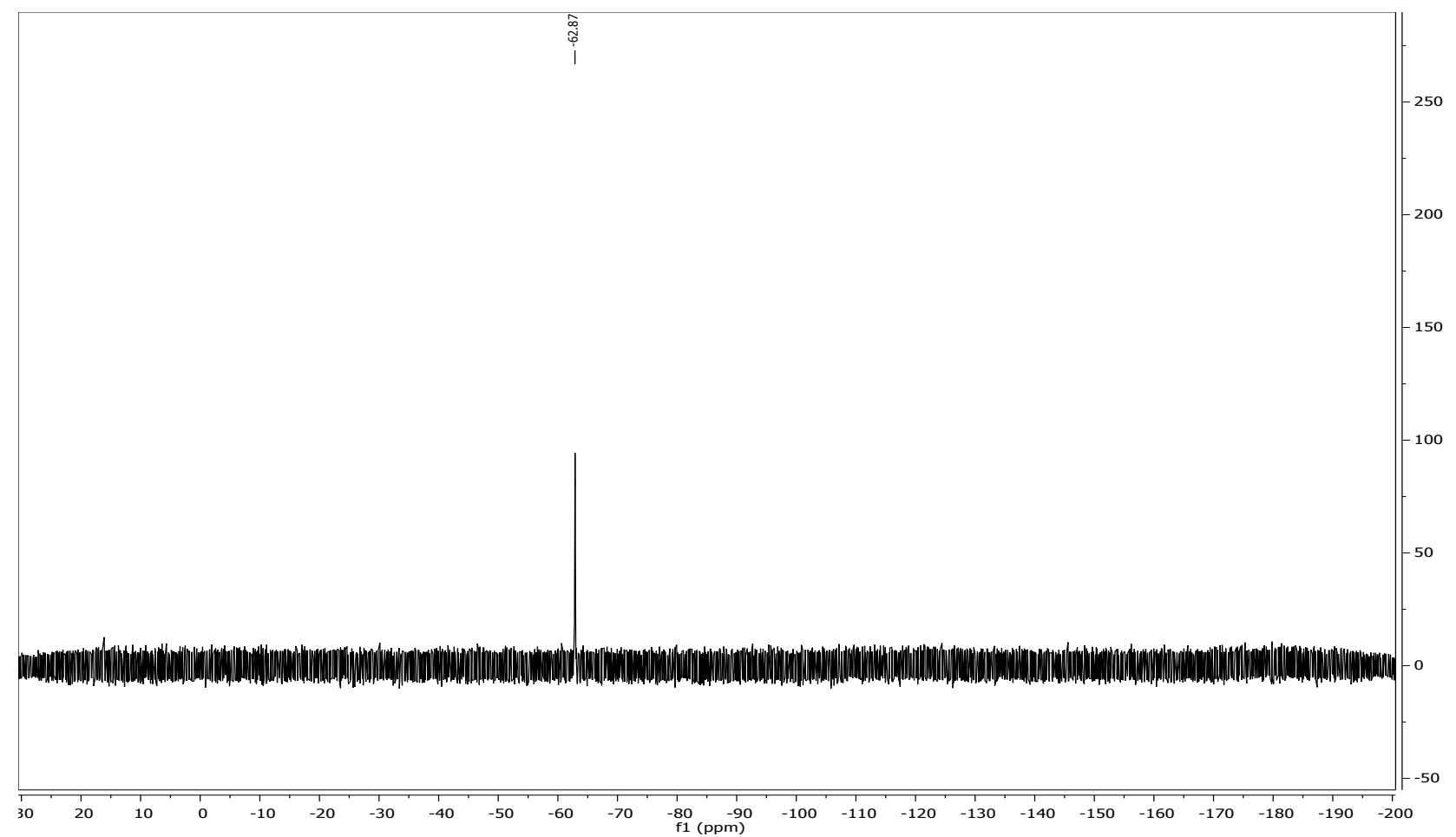


${ }^{1} \mathrm{H}\left(400 \mathrm{MHz}, \mathrm{DMSO}-d_{6}\right),{ }^{13} \mathrm{C}\left(126 \mathrm{MHz}, \mathrm{CDCl}_{3}\right)$ and ${ }^{19} \mathrm{~F}\left(470 \mathrm{MHz}, \mathrm{CDCl}_{3}\right)$ NMR Spectra of 1-(3,5bis(trifluoromethyl)phenyl)-3-((1S,2S)-2-(mesitylselanyl)-2,3-dihydro-1H-inden-1yl)thiourea (10)

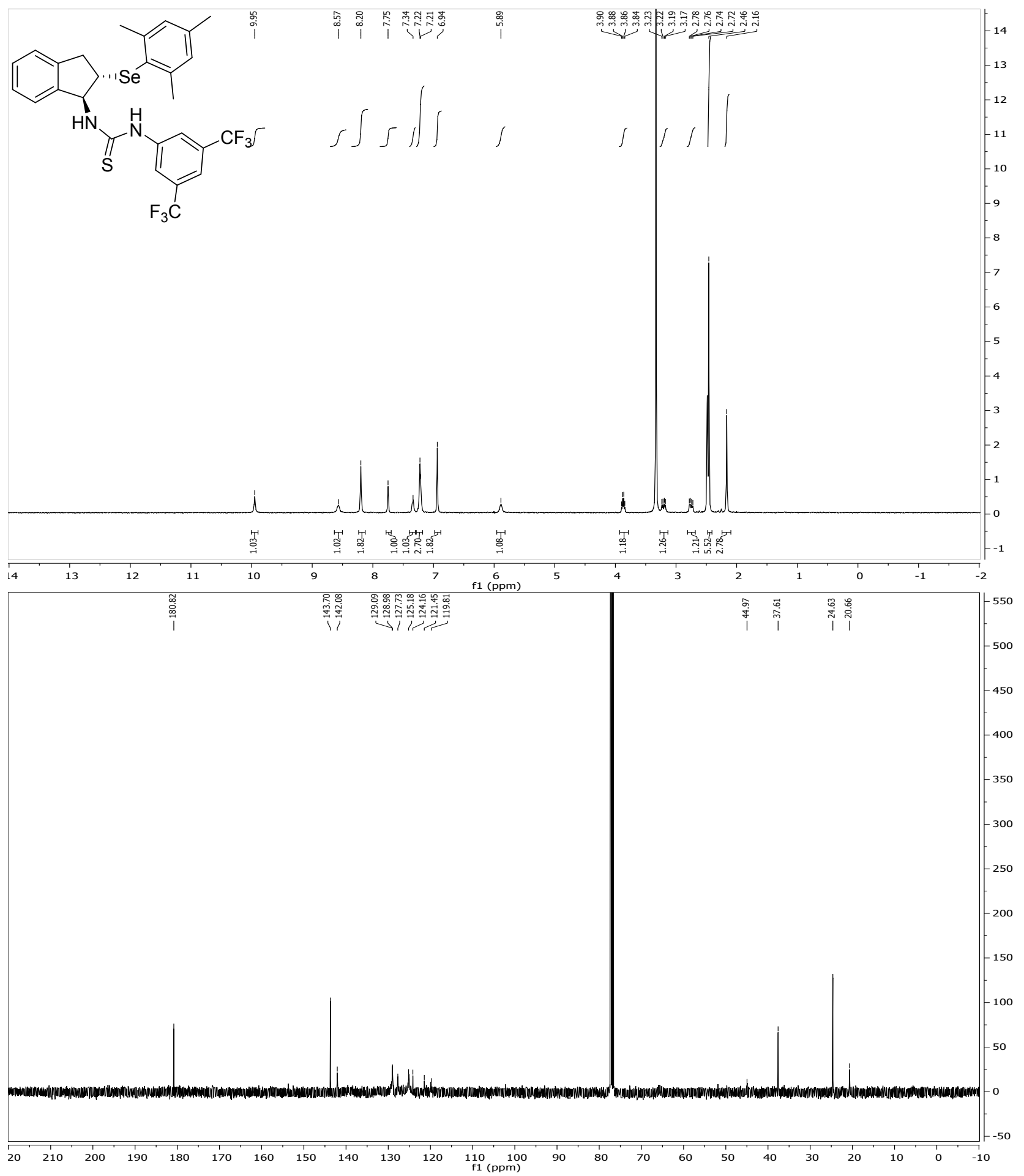




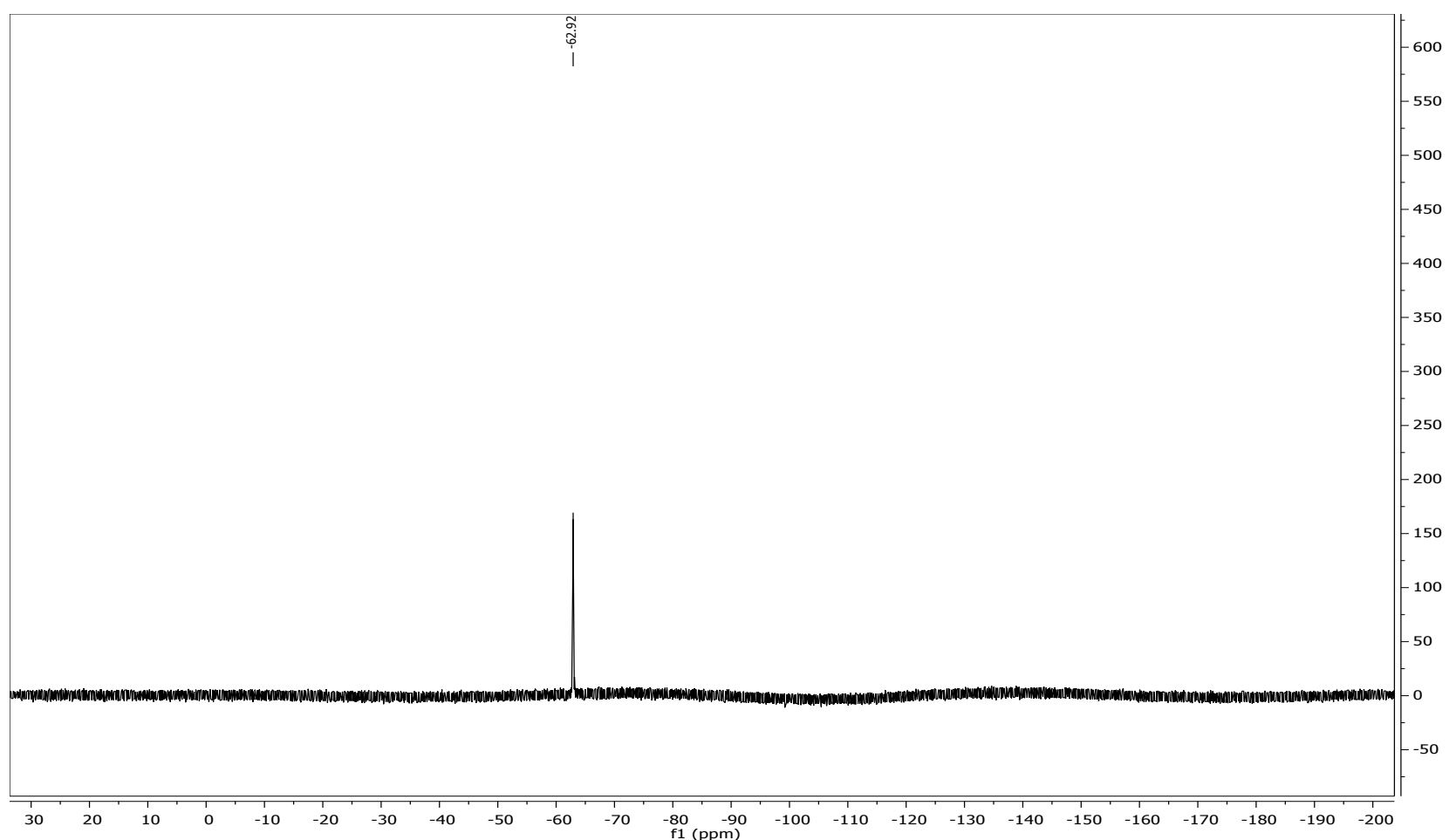


${ }^{1} \mathrm{H}(500 \mathrm{MHz})$ and ${ }^{13} \mathrm{C}(126 \mathrm{MHz}) \mathrm{NMR}$ Spectra $\left(\mathrm{CDCl}_{3}\right)$ of 3-chloro-[1,1'-biphenyl]-2-ol (11b)

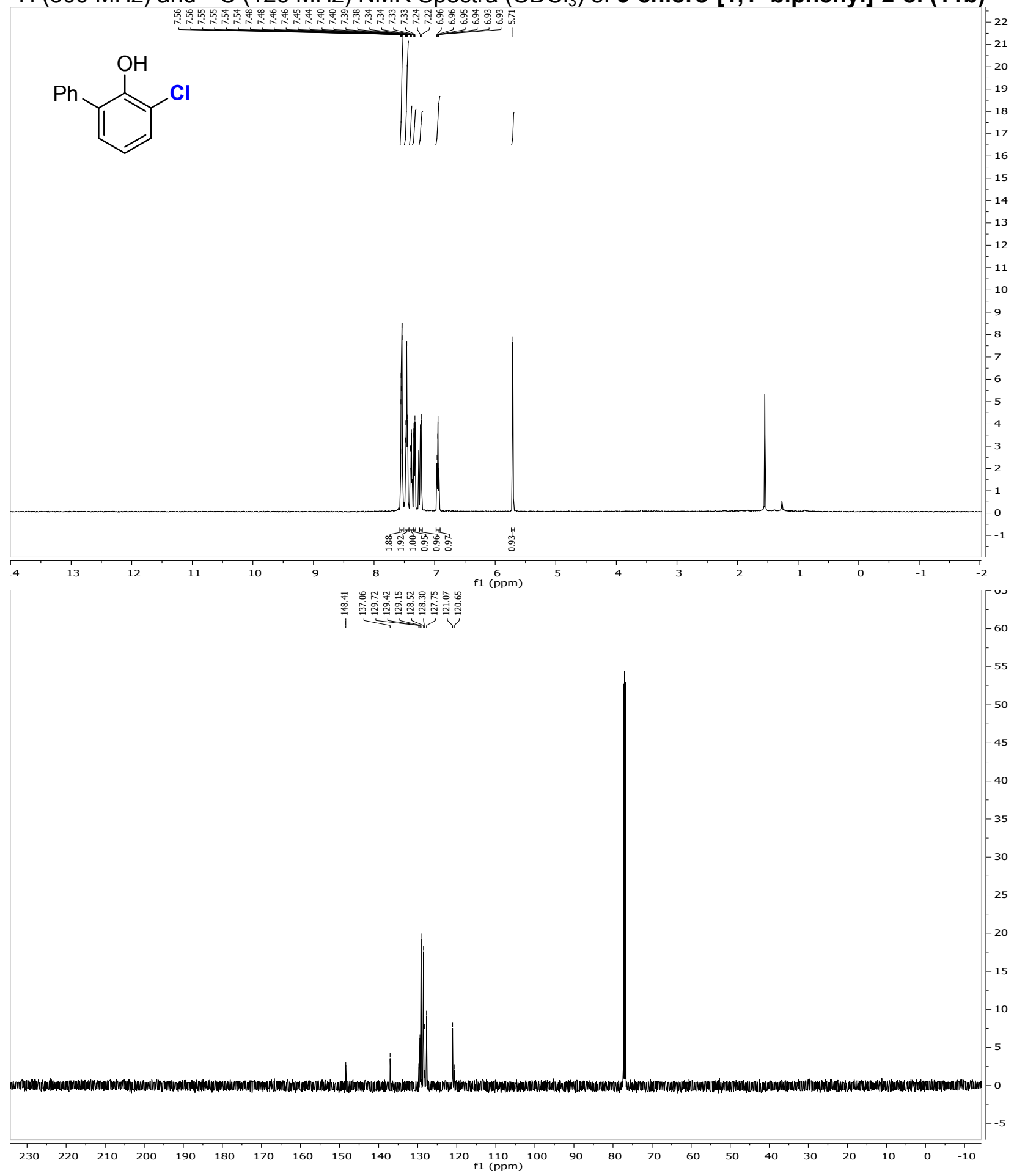


${ }^{1} \mathrm{H}(500 \mathrm{MHz})$ and ${ }^{13} \mathrm{C}(126 \mathrm{MHz})$ NMR Spectra $\left(\mathrm{CDCl}_{3}\right)$ of 3-chloro-2-hydroxybenzonitrile (12b)

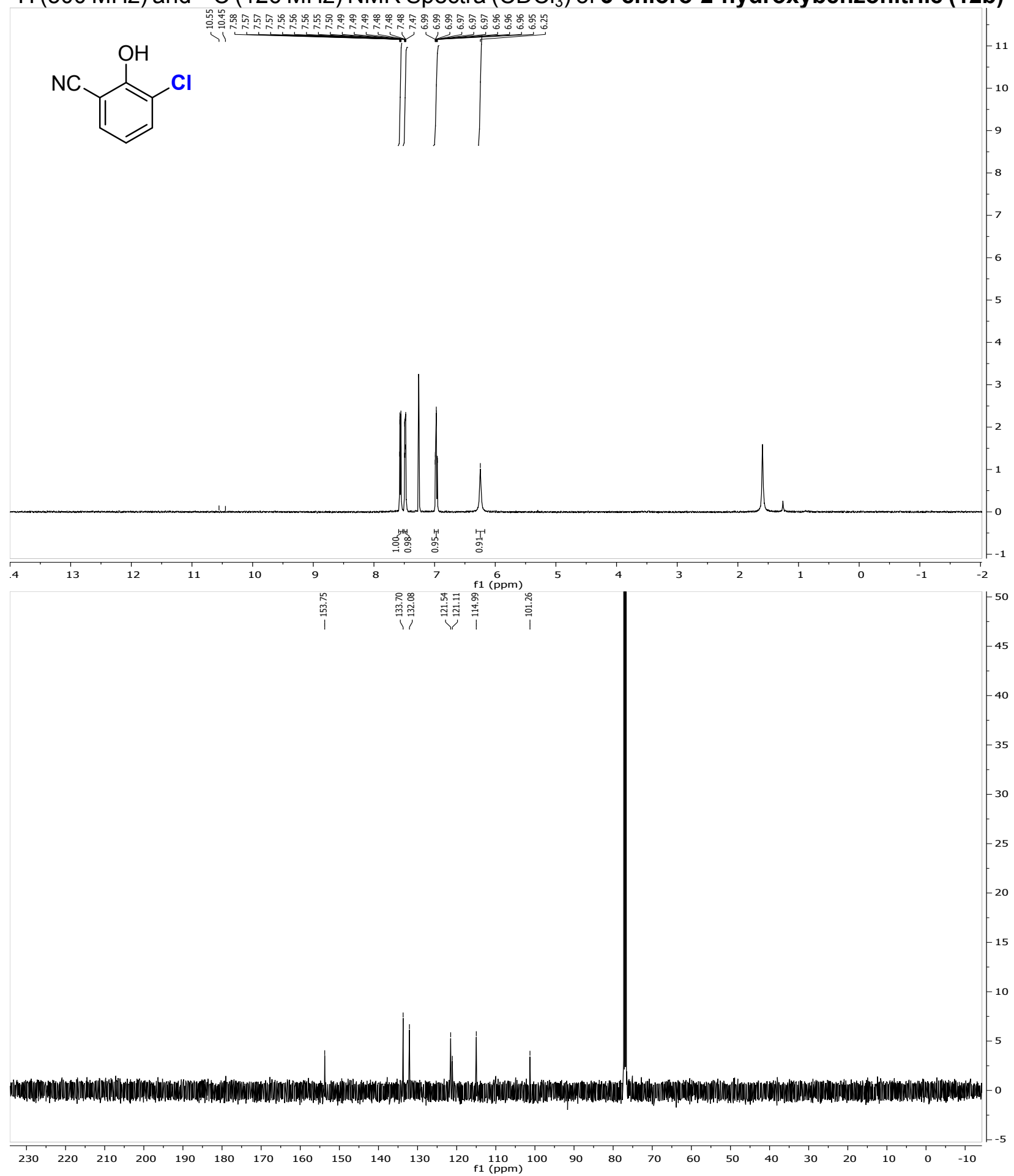


${ }^{1} \mathrm{H}(500 \mathrm{MHz})$ and ${ }^{13} \mathrm{C}(126 \mathrm{MHz}) \mathrm{NMR}$ Spectra $\left(\mathrm{CDCl}_{3}\right)$ of 2-bromo-6-chlorophenol (13b)

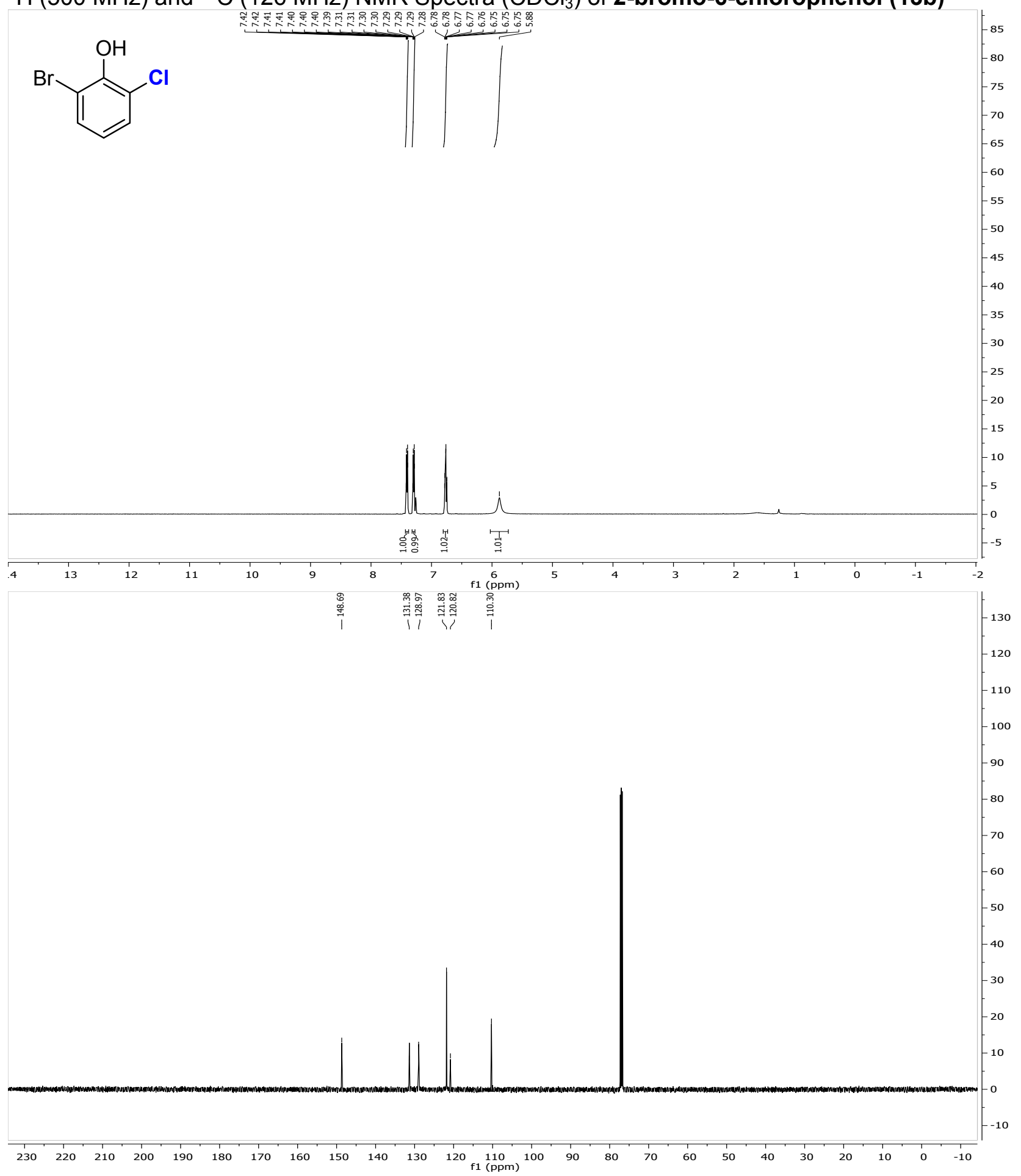


${ }^{1} \mathrm{H}(400 \mathrm{MHz})$ and ${ }^{13} \mathrm{C}(126 \mathrm{MHz}) \mathrm{NMR}$ Spectra $\left(\mathrm{CDCl}_{3}\right)$ of 2-(tert-butyl)-6-chlorophenol (14b)

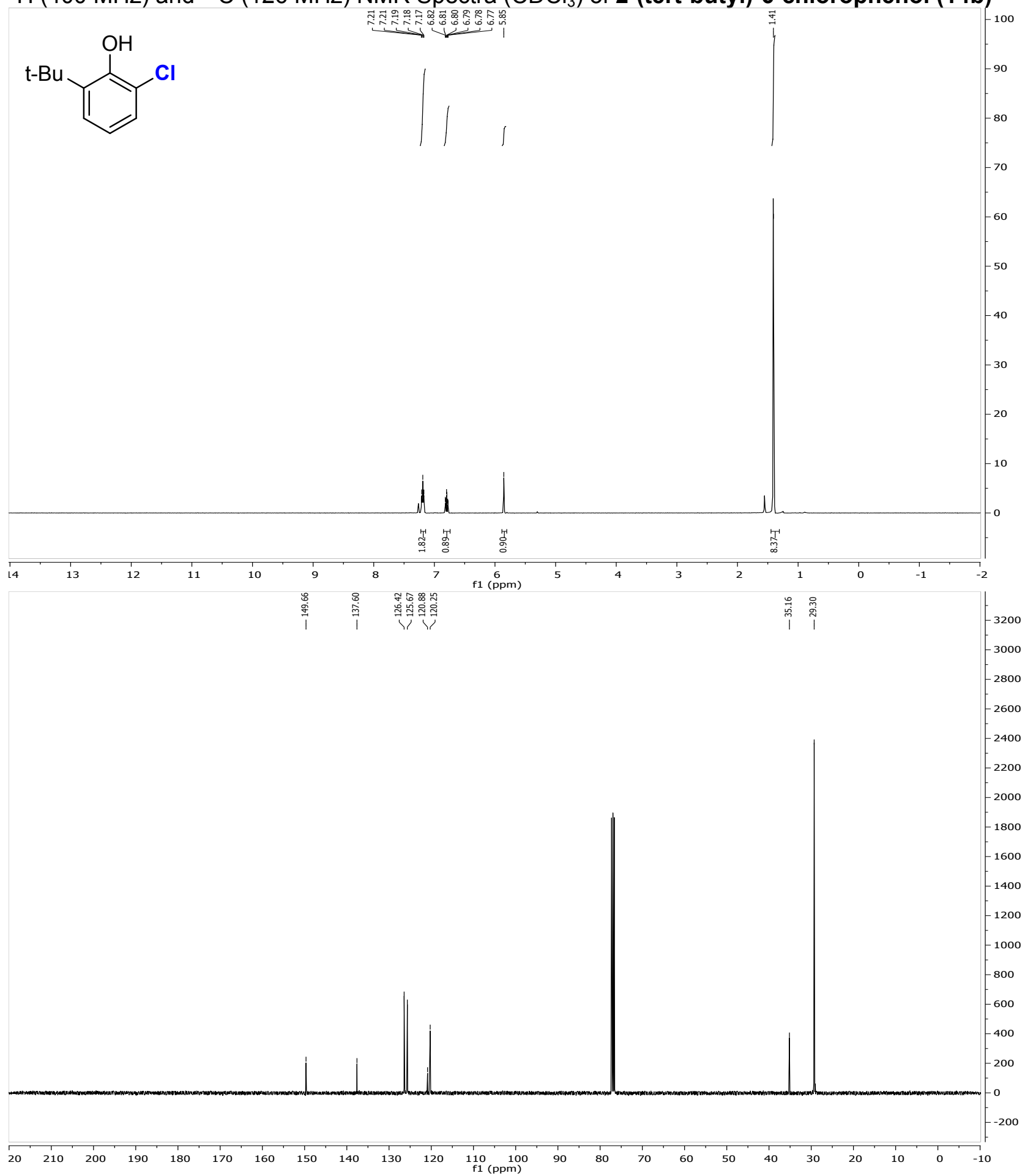


${ }^{1} \mathrm{H}(400 \mathrm{MHz})$ and ${ }^{13} \mathrm{C}(126 \mathrm{MHz}) \mathrm{NMR}$ Spectra $\left(\mathrm{CDCl}_{3}\right)$ of 2-chloro-6-iodophenol (15b)

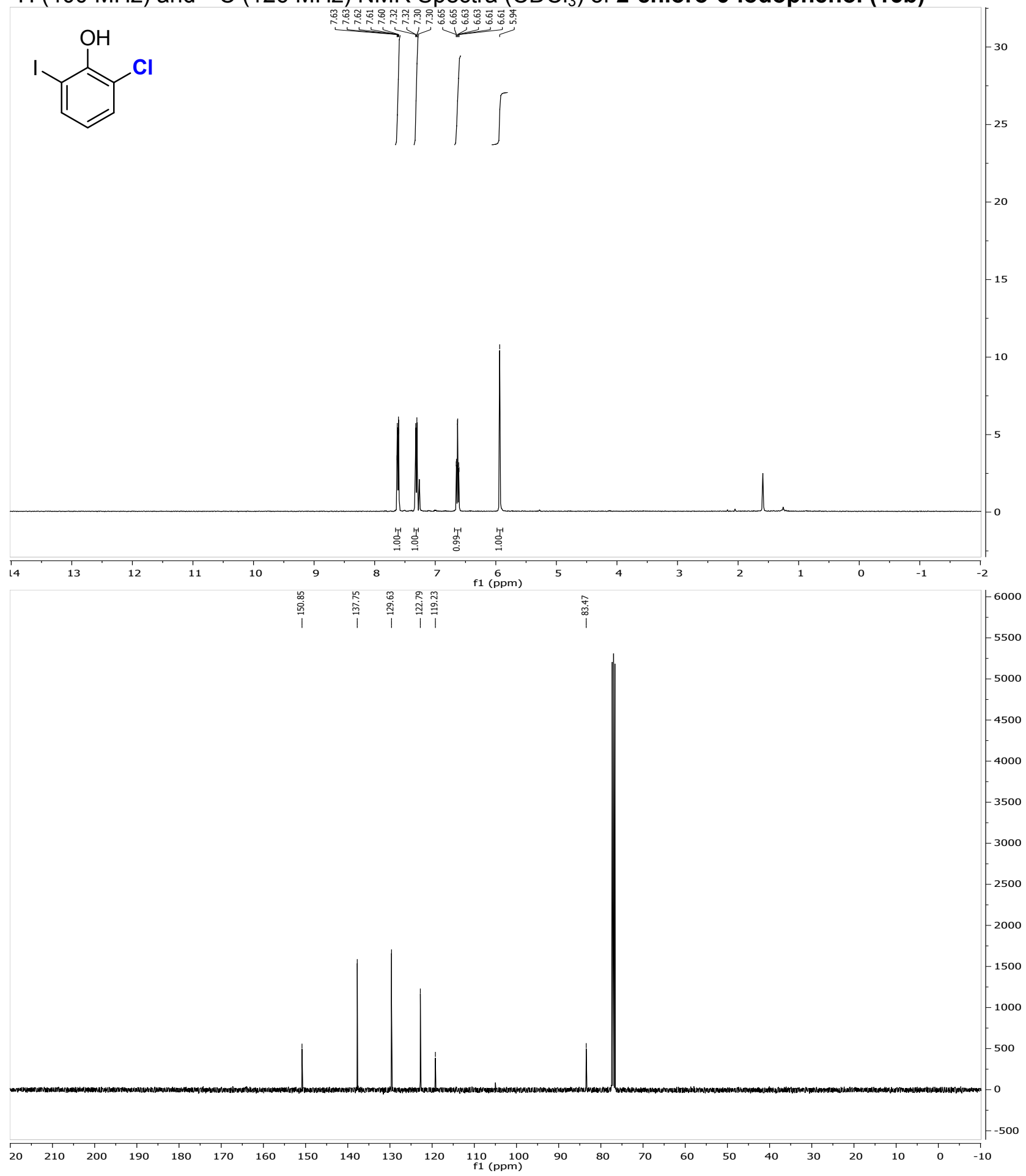


${ }^{1} \mathrm{H}(400 \mathrm{MHz}),{ }^{13} \mathrm{C}(126 \mathrm{MHz})$ and ${ }^{19} \mathrm{~F}(376 \mathrm{MHz})$ NMR Spectra $\left(\mathrm{CDCl}_{3}\right)$ of 2-chloro-5fluorophenol (16b)

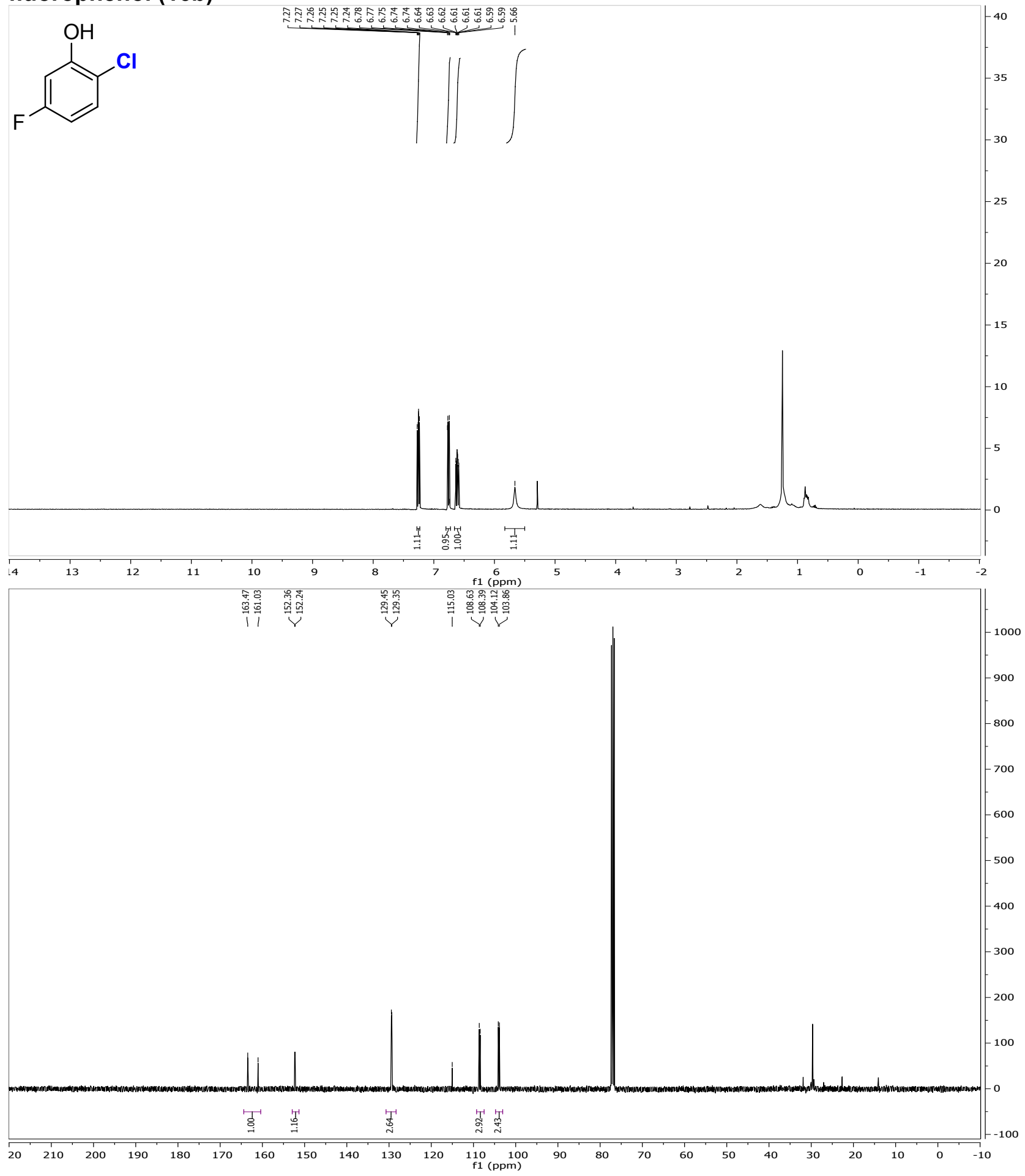




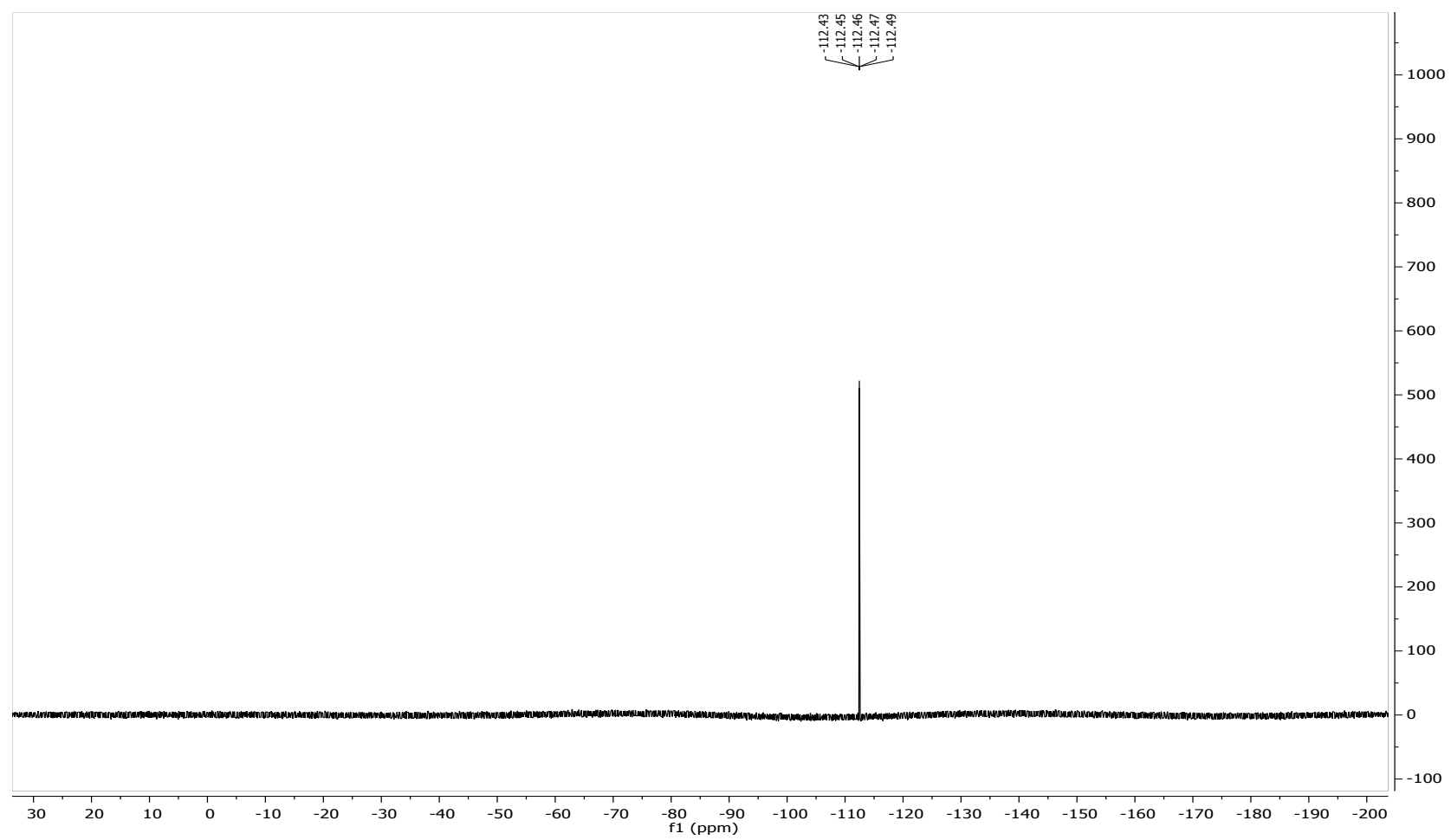


${ }^{1} \mathrm{H}(500 \mathrm{MHz})$ and ${ }^{13} \mathrm{C}(126 \mathrm{MHz}) \mathrm{NMR}$ Spectra $\left(\mathrm{CDCl}_{3}\right)$ of 5-bromo-2-chlorophenol (17b)

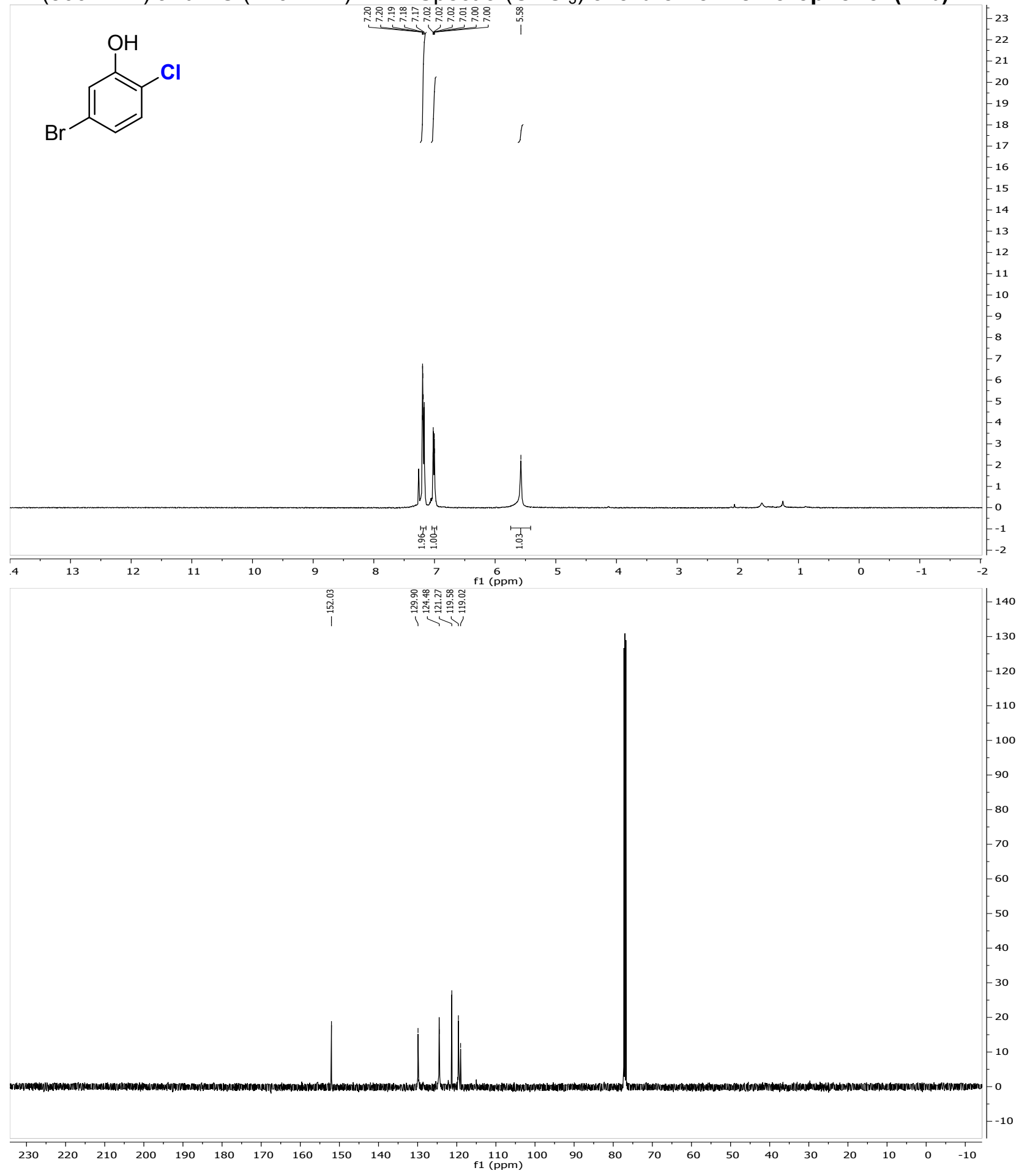


${ }^{1} \mathrm{H}(400 \mathrm{MHz})$ and ${ }^{13} \mathrm{C}(126 \mathrm{MHz})$ NMR Spectra $\left(\mathrm{CDCl}_{3}\right)$ of 4-chloro-[1,1'-biphenyl]-3-ol (18b)

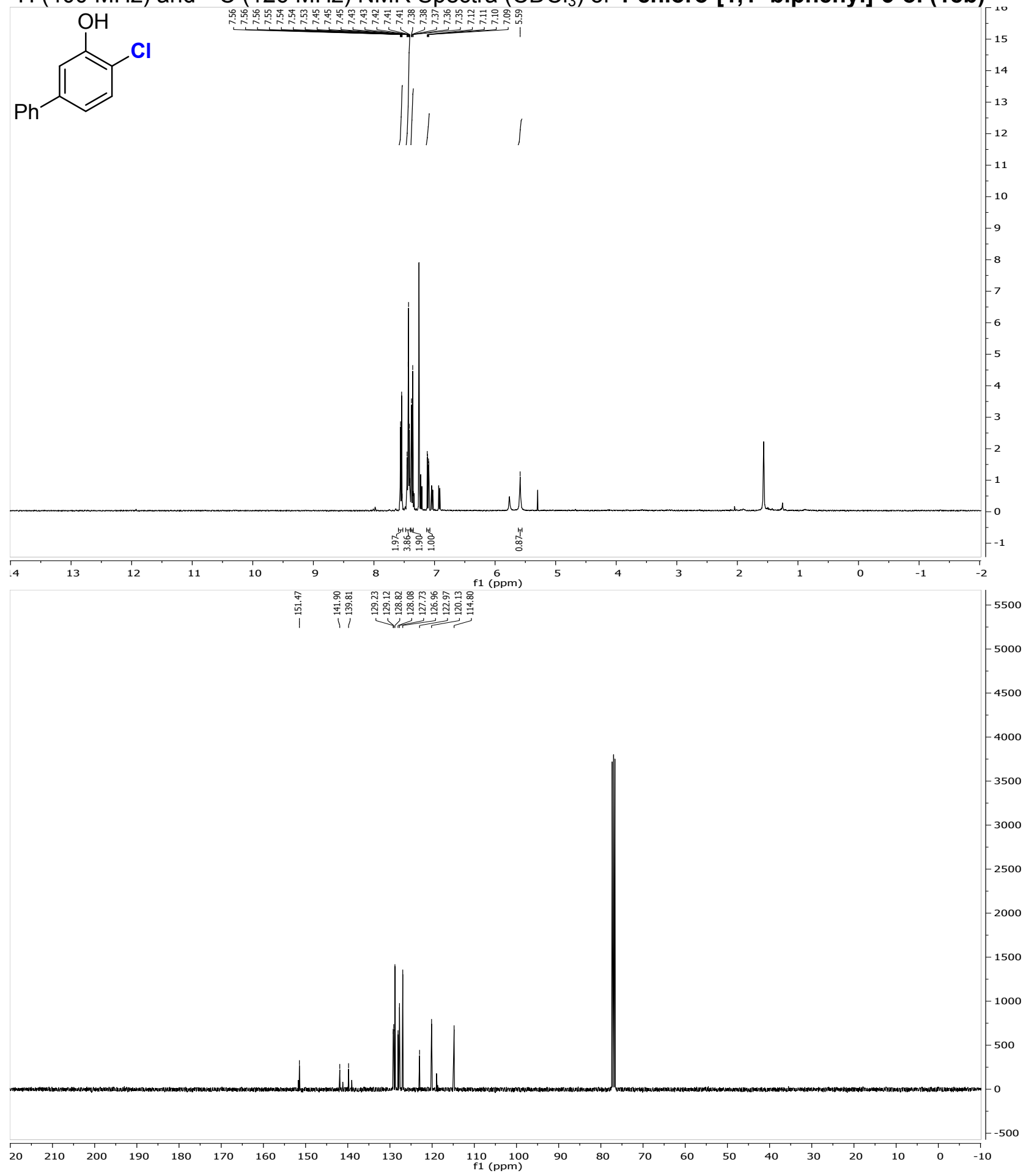


${ }^{1} \mathrm{H}(400 \mathrm{MHz})$ and ${ }^{13} \mathrm{C}(126 \mathrm{MHz})$ NMR Spectra $\left(\mathrm{CDCl}_{3}\right)$ of 2-chloro-6-isopropyl-3methylphenol (19b)

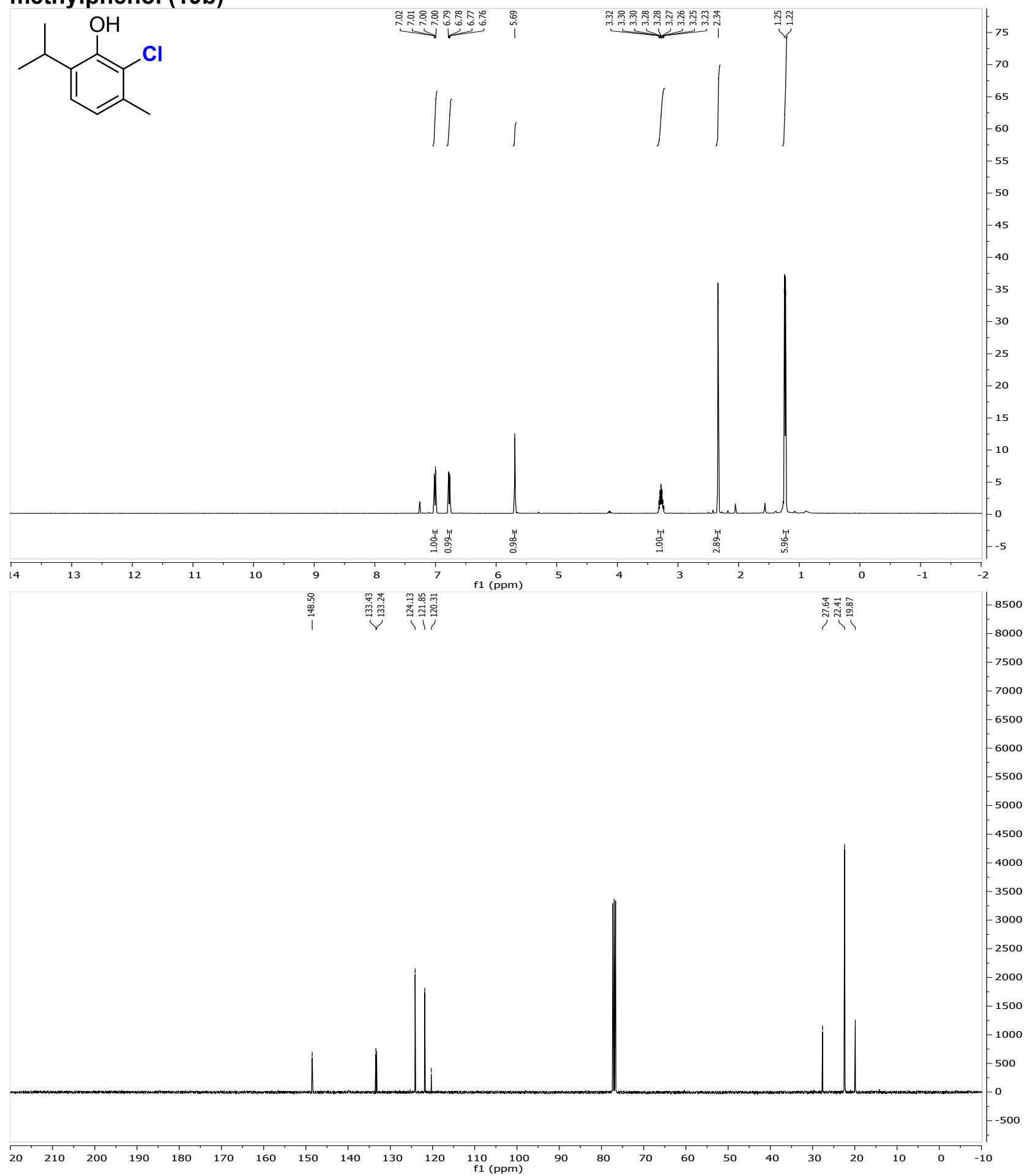


${ }^{1} \mathrm{H}(400 \mathrm{MHz})$ and ${ }^{13} \mathrm{C}(126 \mathrm{MHz})$ NMR Spectra $\left(\mathrm{CDCl}_{3}\right)$ of 2-chloronaphthalen-1-ol (20b)

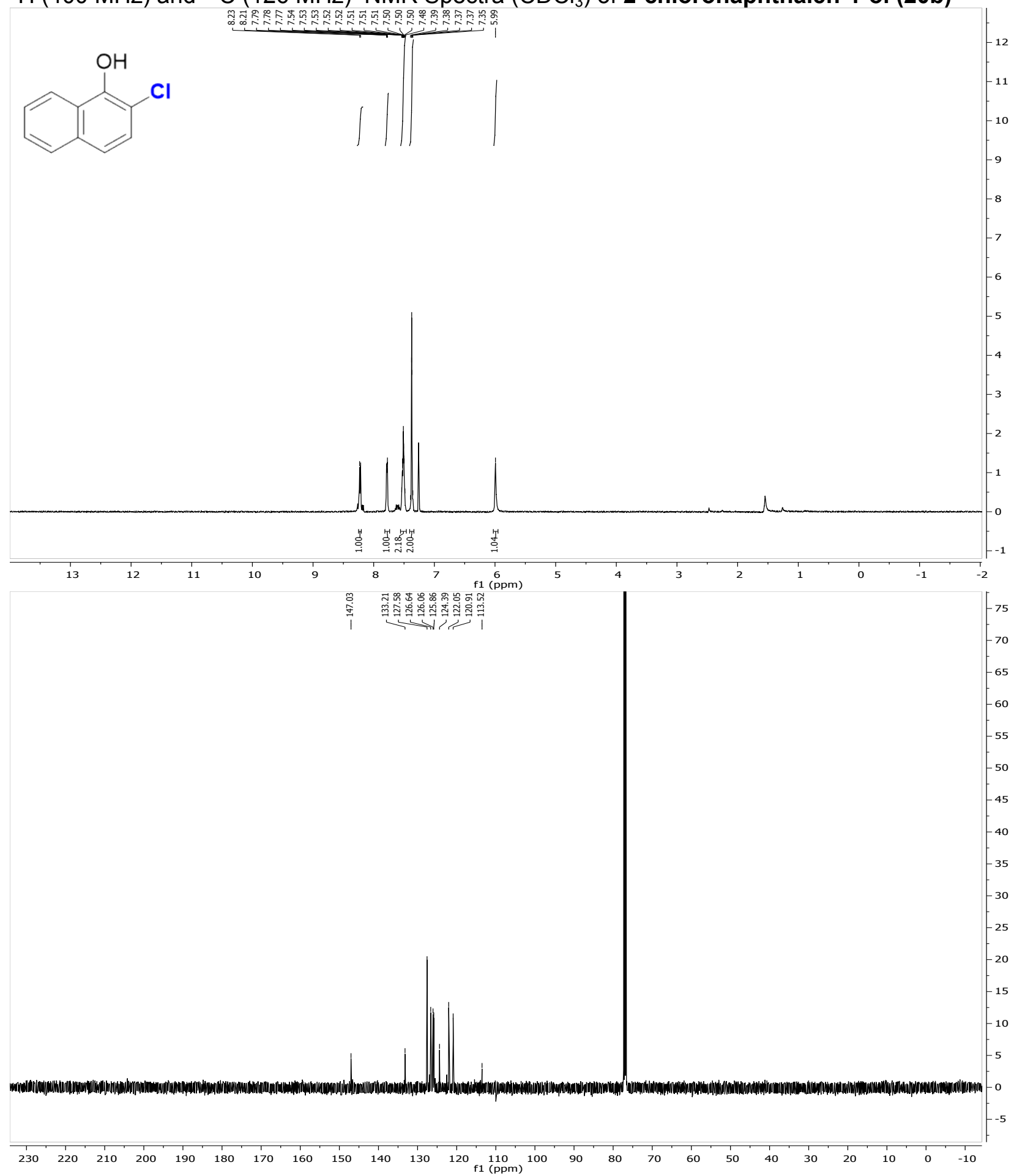


${ }^{1} \mathrm{H}(400 \mathrm{MHz})$ and ${ }^{13} \mathrm{C}(126 \mathrm{MHz}) \mathrm{NMR}$ Spectra $\left(\mathrm{CDCl}_{3}\right)$ of 2-chloro-6-methylaniline (21b)

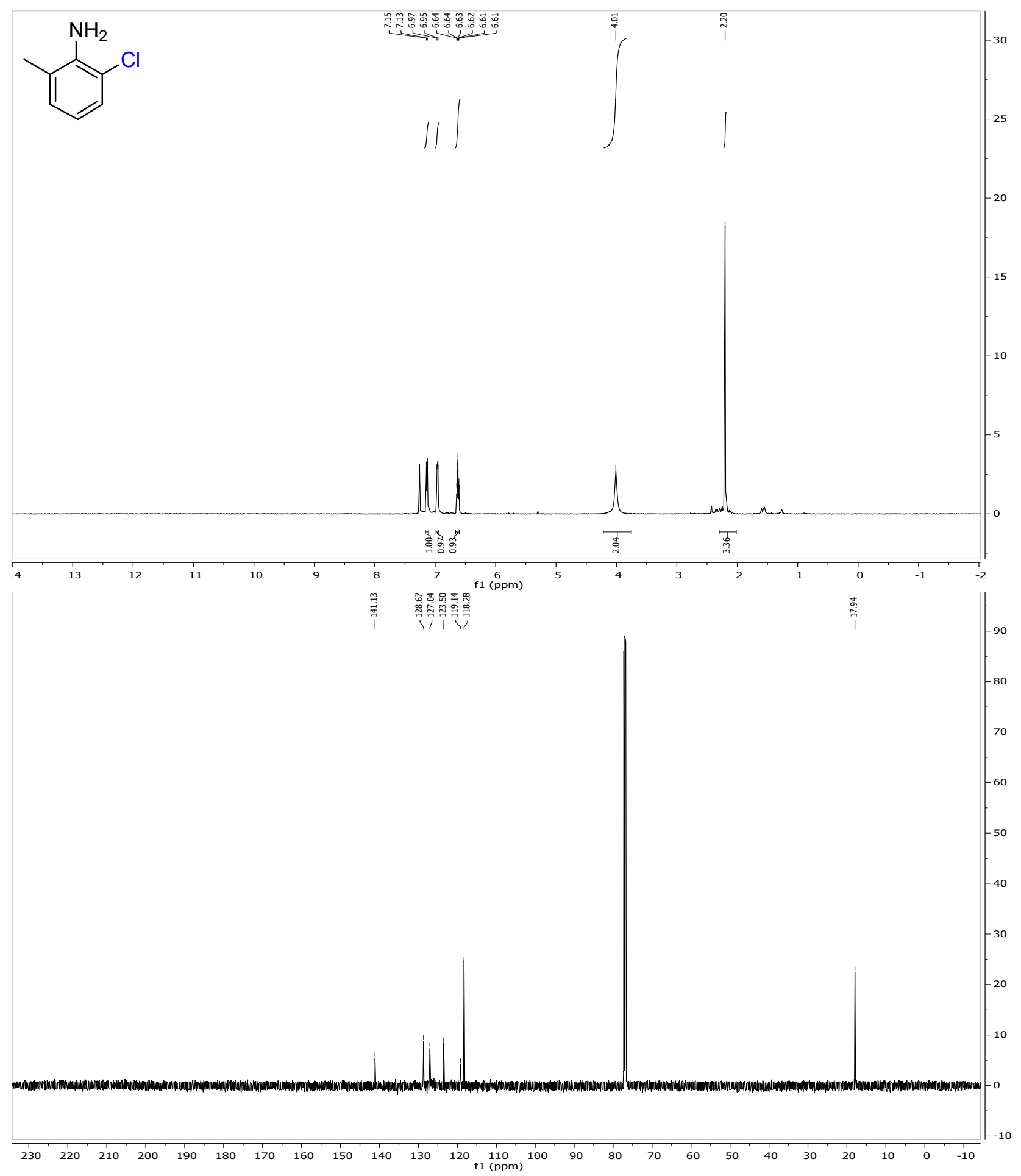


${ }^{1} \mathrm{H}(400 \mathrm{MHz})$ and ${ }^{13} \mathrm{C}(126 \mathrm{MHz})$ NMR Spectra $\left(\mathrm{CDCl}_{3}\right)$ of 2-chloro-6-isopropylaniline (22b)

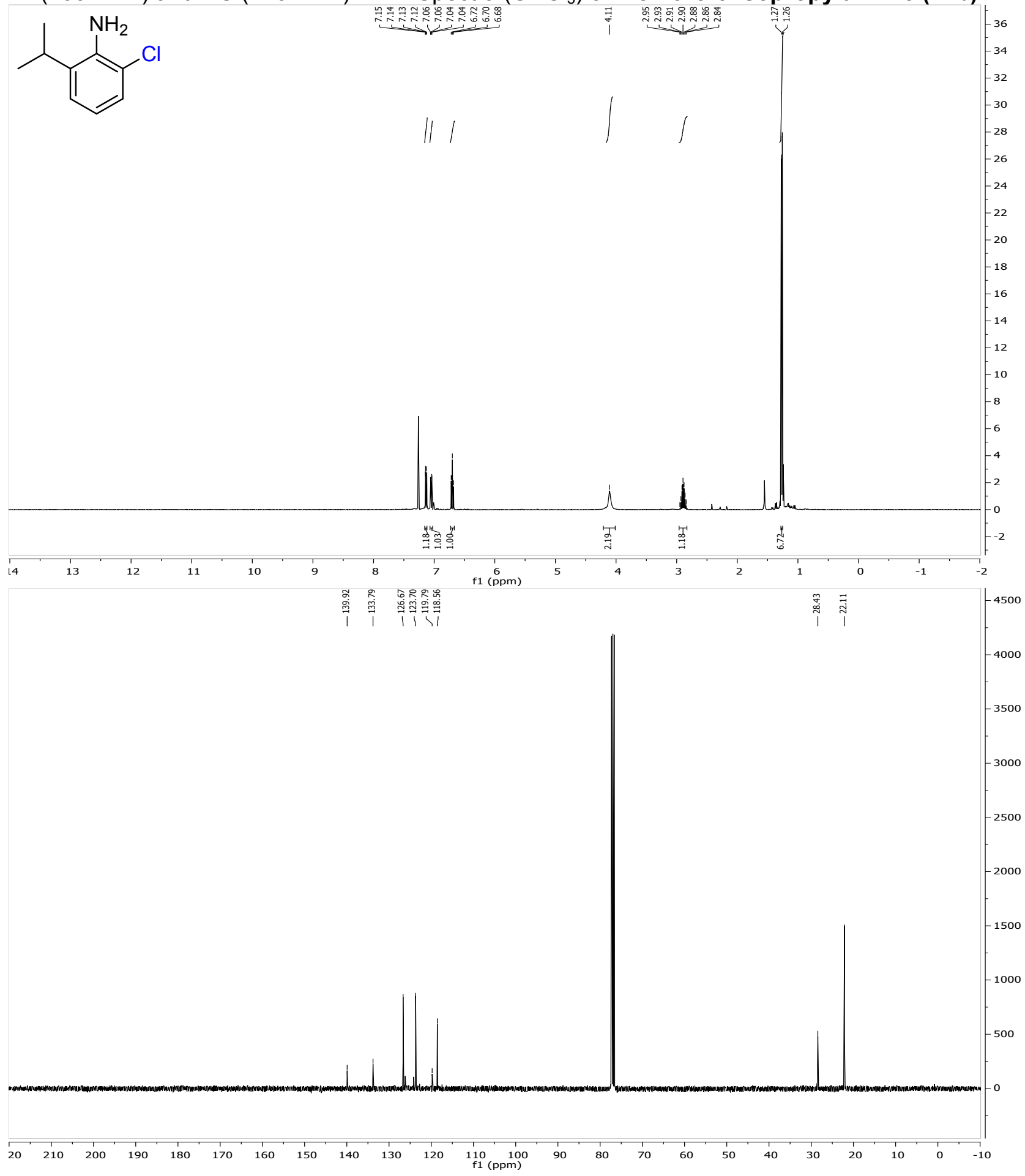


${ }^{1} \mathrm{H}(400 \mathrm{MHz})$ and ${ }^{13} \mathrm{C}(126 \mathrm{MHz}) \mathrm{NMR}$ Spectra $\left(\mathrm{CDCl}_{3}\right)$ of 2-(tert-butyl)-6-chloroaniline (23b)

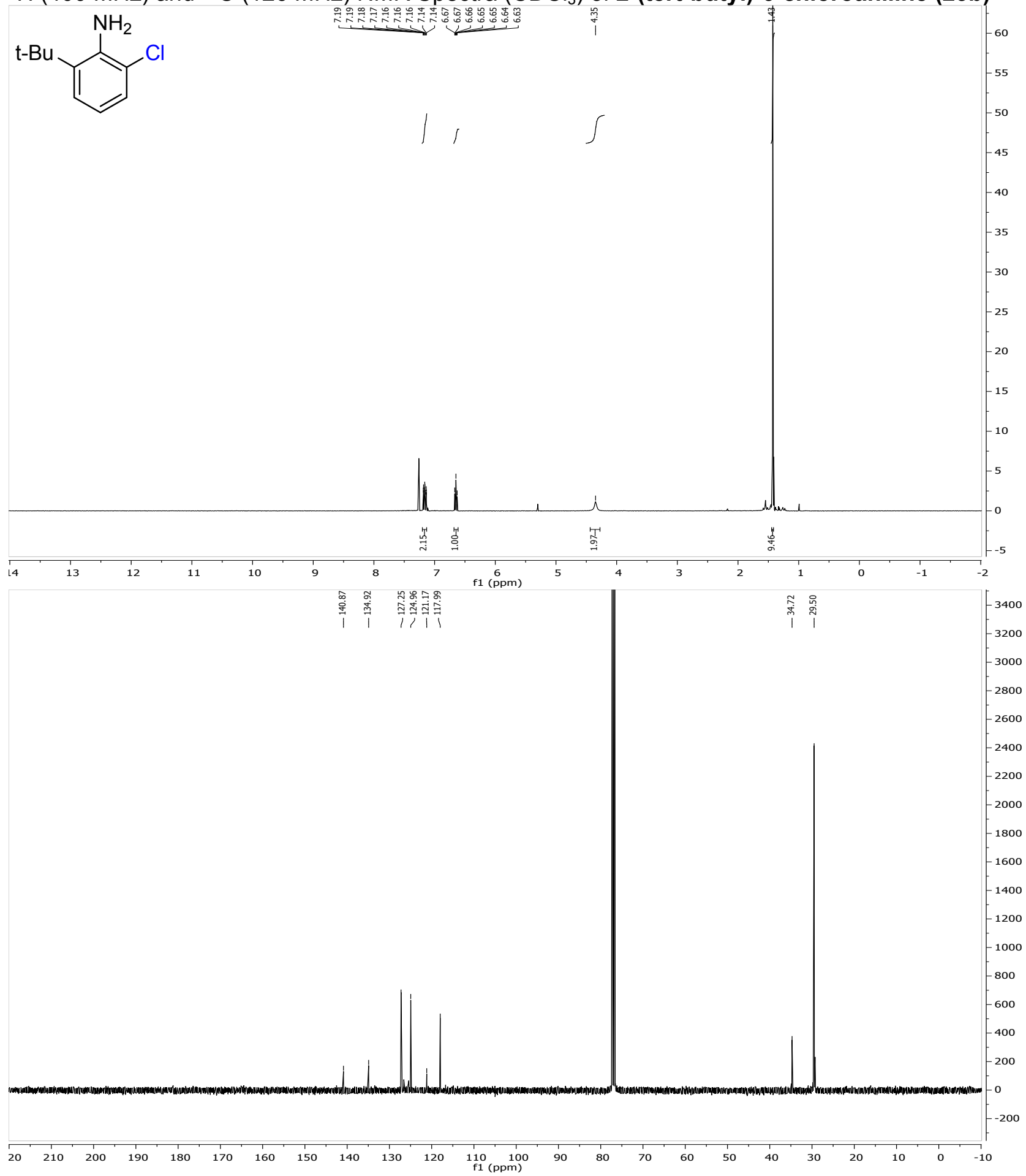


${ }^{1} \mathrm{H}(400 \mathrm{MHz})$ and ${ }^{13} \mathrm{C}(126 \mathrm{MHz}) \mathrm{NMR}$ Spectra $\left(\mathrm{CDCl}_{3}\right)$ of 2-bromo-6-chloroaniline (24b)

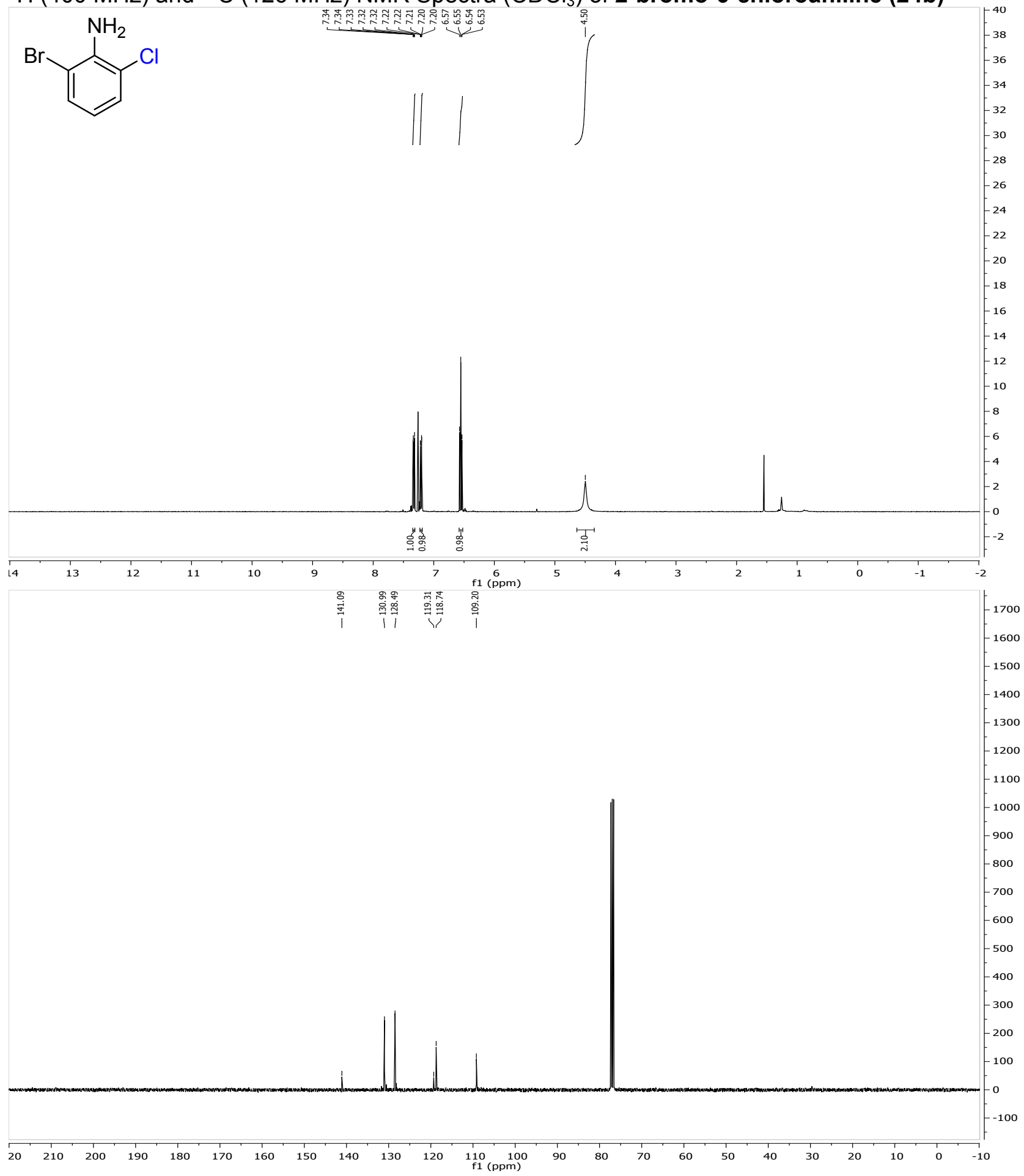


${ }^{1} \mathrm{H}(400 \mathrm{MHz}),{ }^{13} \mathrm{C}(126 \mathrm{MHz})$ and ${ }^{19} \mathrm{~F}(376 \mathrm{MHz})$ NMR Spectra $\left(\mathrm{CDCl}_{3}\right)$ of 2-chloro-5fluoroaniline (25b)

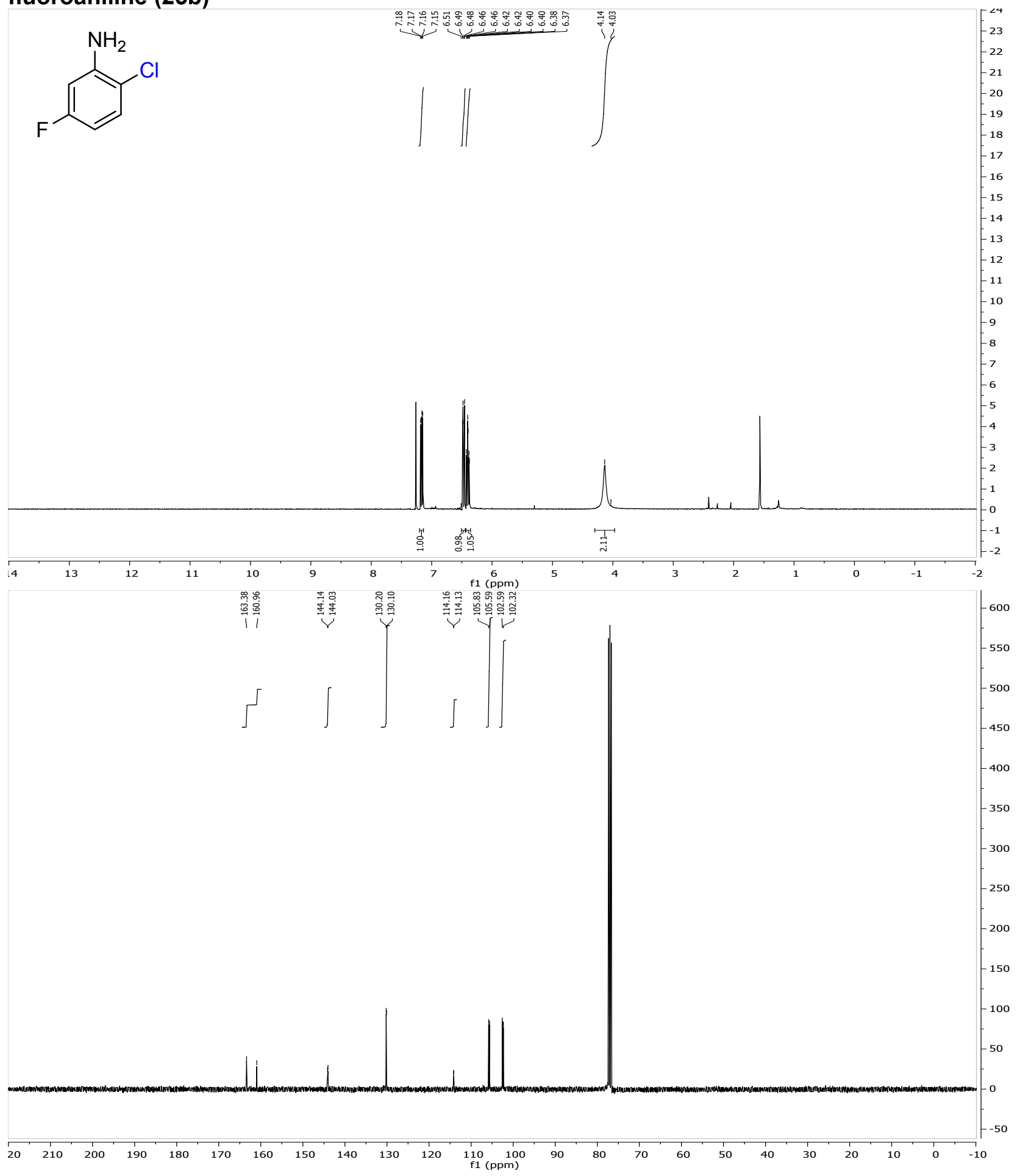




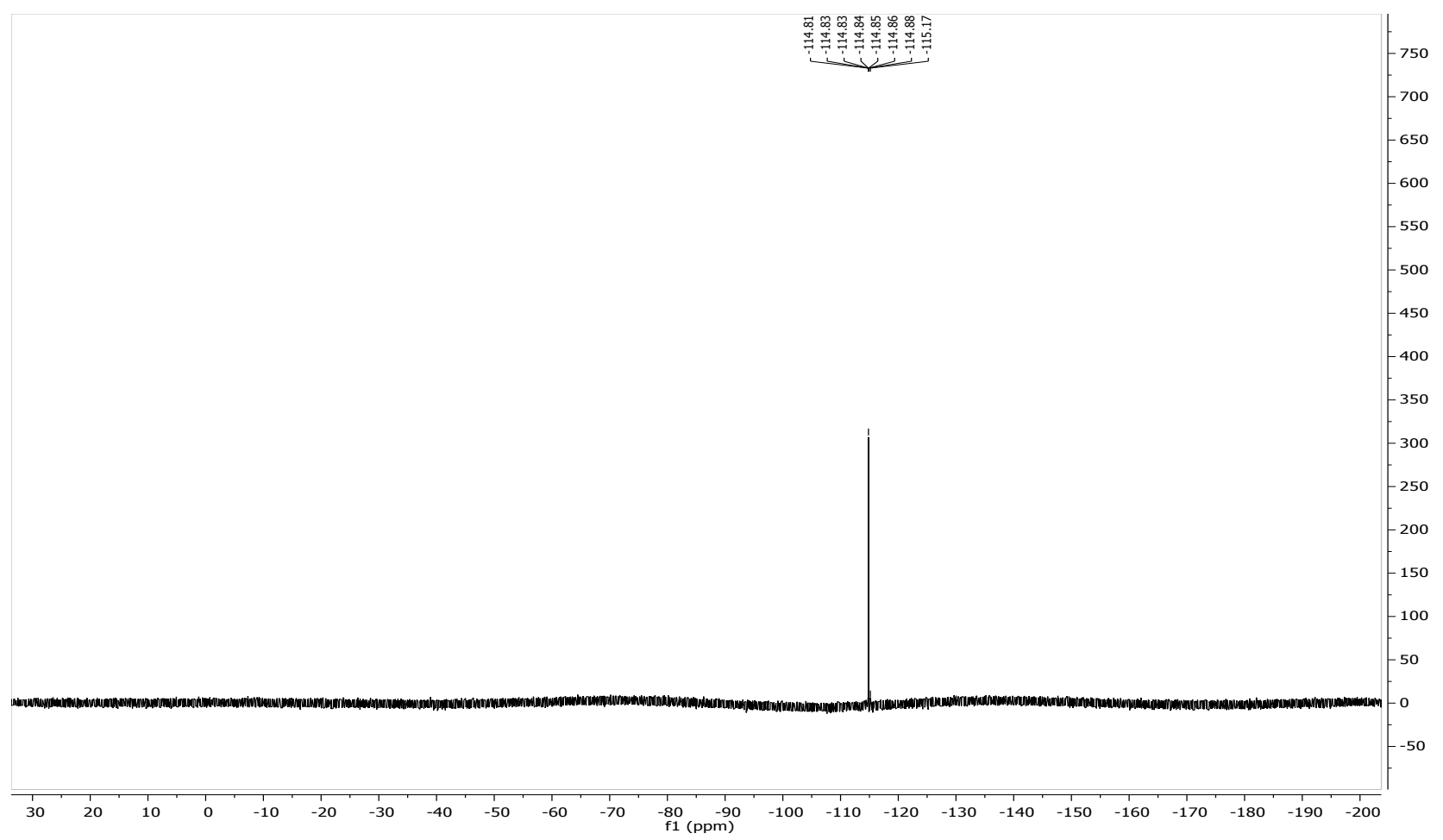


${ }^{1} \mathrm{H}(400 \mathrm{MHz})$ and ${ }^{13} \mathrm{C}(126 \mathrm{MHz}) \mathrm{NMR}$ Spectra $\left(\mathrm{CDCl}_{3}\right)$ of 5-bromo-2-chloroaniline (26b)

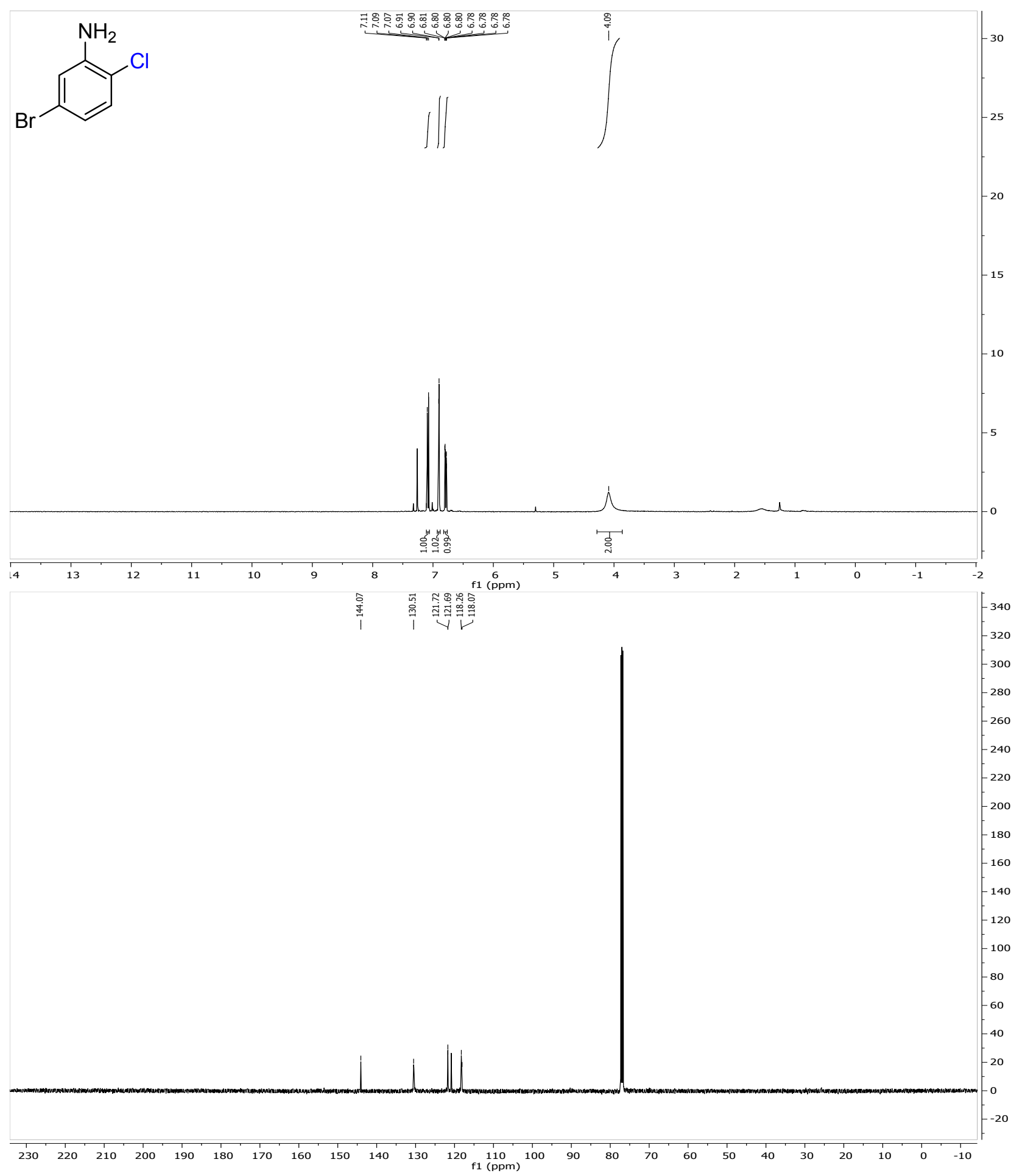


${ }^{1} \mathrm{H}(400 \mathrm{MHz})$ and ${ }^{13} \mathrm{C}(126 \mathrm{MHz}) \mathrm{NMR}$ Spectra $\left(\mathrm{CDCl}_{3}\right)$ of 4-chloro-[1,1'-biphenyl]-3-amine (27b). There is an impurity of starting material in this spectrum.

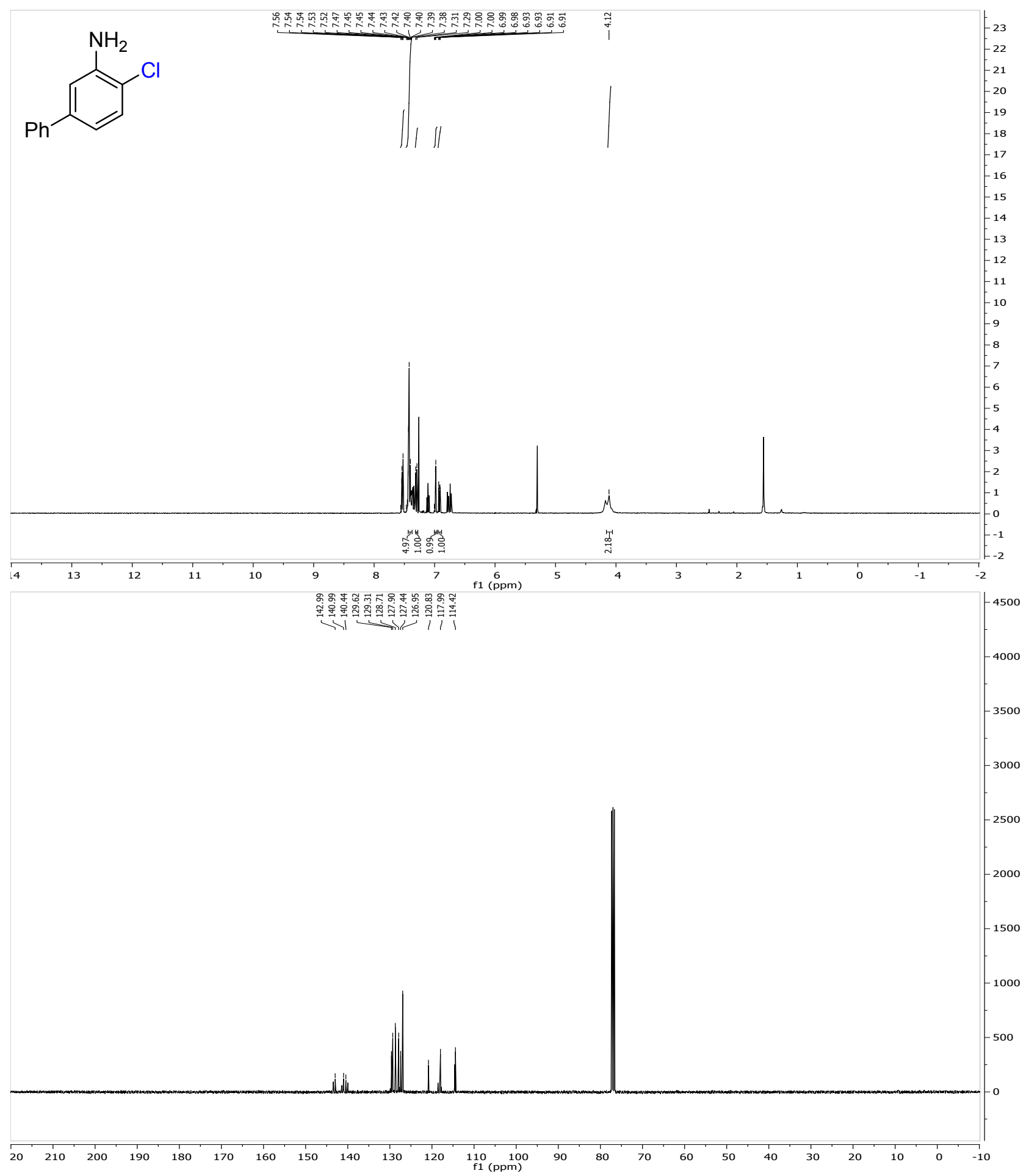




\section{Crystal Structure Experimental Summary}

Single crystals of compound 1a were obtained by slow solvent evaporation from dichloromethane solution over the course of three days. The single crystal X-ray diffraction studies were carried out on a Bruker Kappa APEX-II CCD diffractometer equipped with $\mathrm{Cu} \mathrm{K}_{\alpha}$ radiation $(\lambda=1.5478)$. A $0.097 \times 0.035 \times 0.021 \mathrm{~mm}$ piece of a colorless needle was mounted on a Cryoloop with Paratone oil. Data were collected in a nitrogen gas stream at $100(2) \mathrm{K}$ using $\phi$ and $\varpi$ scans. Crystal-to-detector distance was $45 \mathrm{~mm}$ using variable exposure time (10s-60s) depending on $\theta$ with a scan width of $2.0^{\circ}$. Data collection was $98.8 \%$ complete to $68.00^{\circ}$ in $\theta$. A total of 12255 reflections were collected covering the indices, $-12<=h<=12,-18<=k<=18,-10<=\mid<=12.5851$ reflections were found to be symmetry independent, with a Rint of 0.0725 . Indexing and unit cell refinement indicated a primitive, monoclinic lattice. The space group was found to be $P 21$. The data were integrated using the Bruker SAINT software program and scaled using the SADABS software program. Solution by direct methods (SHELXT) produced a complete phasing model consistent with the proposed structure.

All nonhydrogen atoms were refined anisotropically by full-matrix least-squares (SHELXL-2014). All carbon bonded hydrogen atoms were placed using a riding model. Their positions were constrained relative to their parent atom using the appropriate HFIX command in SHELXL-2014. All other hydrogen atoms (H-bonding) were located in the difference map. Their relative positions were restrained using DFIX commands and their thermals freely refined. The absolute stereochemistry of the molecule was established by anomalous dispersion using the Parson's method with a Flack parameter of 0.041(43).

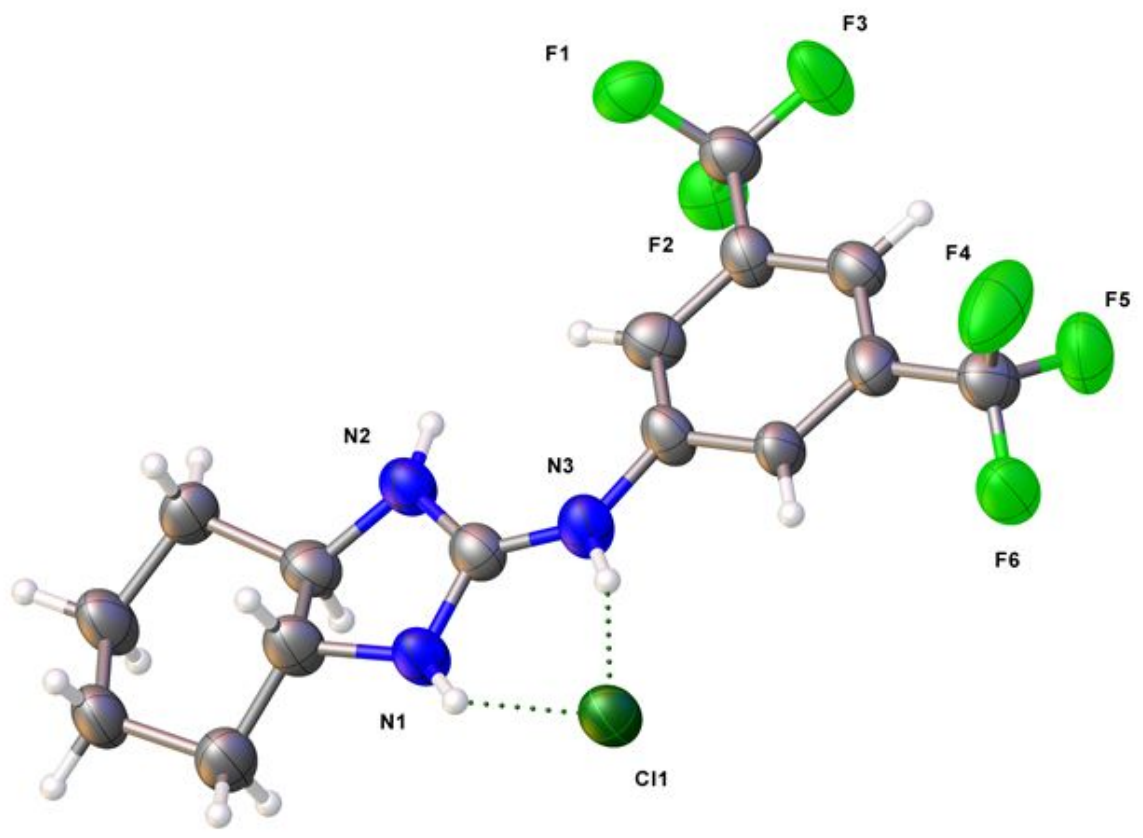

X-Ray Structure of catalyst $1 \mathrm{a}$ degradation product (guanidinium salt), showing thermal ellipsoids at the $50 \%$ probability level. 
Crystal data and structure refinement for catalyst $1 \mathrm{a}$.

Report date

Identification code

Empirical formula

Molecular formula

Formula weight

Temperature

Wavelength

Crystal system

Space group

Unit cell dimensions

Volume

Z

Density (calculated)

Absorption coefficient

$\mathrm{F}(000)$

Crystal size

Crystal color, habit

Theta range for data collection

Index ranges

Reflections collected

Independent reflections

Completeness to theta $=68.000^{\circ}$

Absorption correction

Max. and min. transmission

Refinement method

Data / restraints / parameters

Goodness-of-fit on F2

Final $\mathrm{R}$ indices [l>2sigma(I)]

$\mathrm{R}$ indices (all data)

Absolute structure parameter

Extinction coefficient

Largest diff. peak and hole
2018-06-12

SMM-11-141-A-B

C15 H16 Cl F6 N3

C15 H16 F6 N3, Cl

387.76

$100.0 \mathrm{~K}$

$1.54178 \AA$

Monoclinic

P 1211

$a=10.5346(5) \AA$

$\alpha=90^{\circ}$.

$b=15.4968(7) \AA$

$\beta=110.950(4)^{\circ}$.

$c=10.7550(6) \AA$

$\gamma=90^{\circ}$.
4

$1.571 \mathrm{Mg} / \mathrm{m}^{3}$

$2.709 \mathrm{~mm}^{-1}$

792

$0.097 \times 0.035 \times 0.021 \mathrm{~mm}^{3}$

Colorless Needle

4.402 to $68.556^{\circ}$.

$-12<=\mathrm{h}<=12,-18<=\mathrm{k}<=18,-10<=\mathrm{l}<=12$

12255

$5851[R($ int $)=0.0725, R($ sigma $)=0.1130]$

$98.8 \%$

Semi-empirical from equivalents

0.3200 and 0.1915

Full-matrix least-squares on $\mathrm{F}^{2}$

5851 / 7 / 475

1.025

$\mathrm{R} 1=0.0638, w R 2=0.1525$

$\mathrm{R} 1=0.1016, \mathrm{wR} 2=0.1775$

$0.04(4)$

$\mathrm{n} / \mathrm{a}$

0.441 and -0.329 e. $\AA^{-3}$ 


\section{Isotopically Labeled Experiments with Lewis Base Catalysts}

${ }^{13} \mathrm{C}$ and ${ }^{15} \mathrm{~N}$-labeled Experiments with Nagasawa's Bis-Thiourea Catalyst

Both isotopically labeled derivatives of catalyst 1 were synthesized. Then a $.025 \mathrm{M}$ solution of catalyst was dissolved in $\mathrm{CDCl}_{3}$ in an NMR tube. $\mathrm{A} \mathrm{t}=0$ was taken, followed by addition of NCS reagent. After 10 minutes, another spectrum was taken. For proton and carbon, phenol was added after completion of the $2^{\text {nd }} 10$ minute scan and another spectrum was taken afterwards. For nitrogen, the reaction was left for two days and then quenched with phenol.

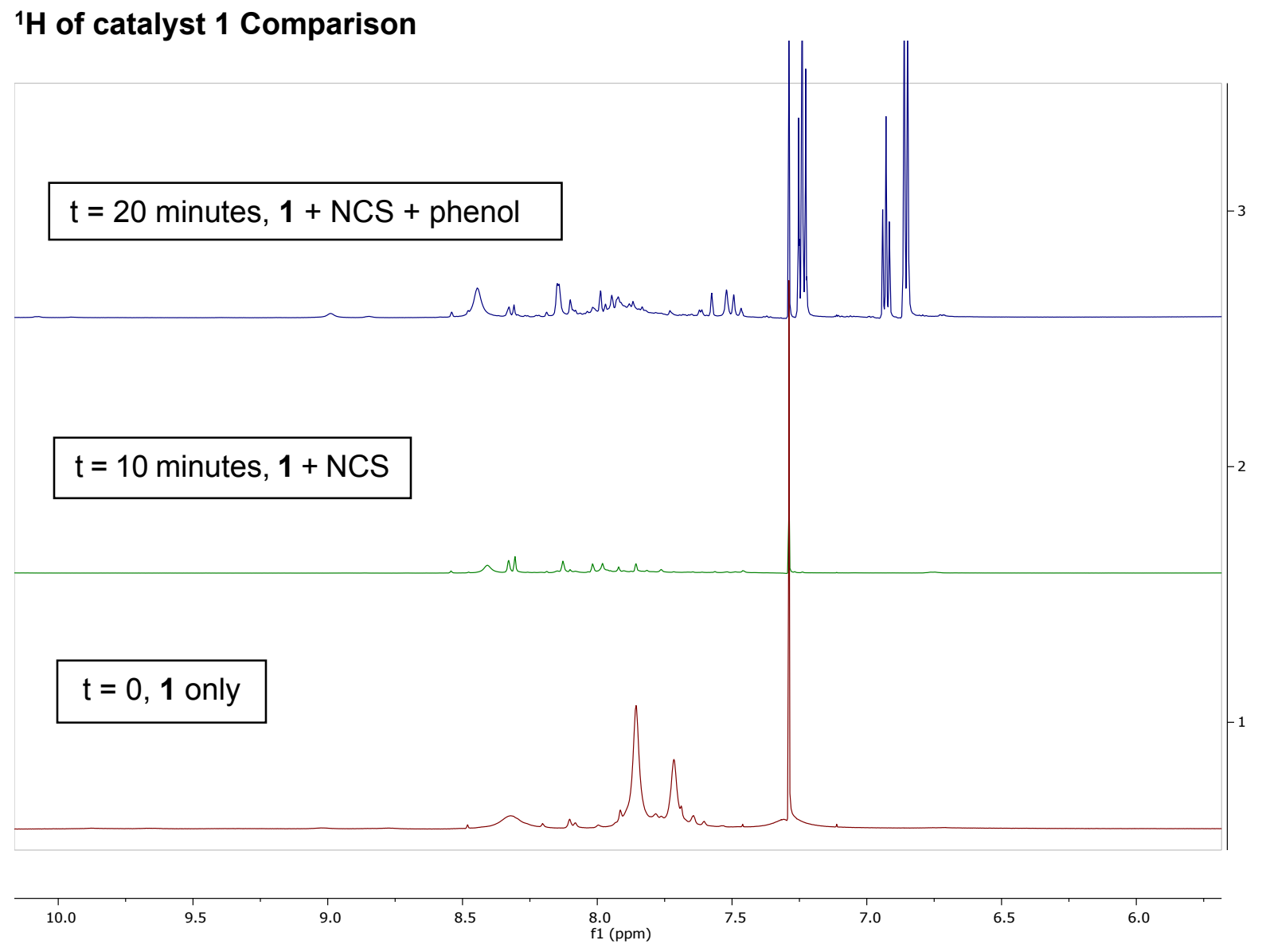


${ }^{13} \mathrm{C}$ of catalyst 1 Comparison

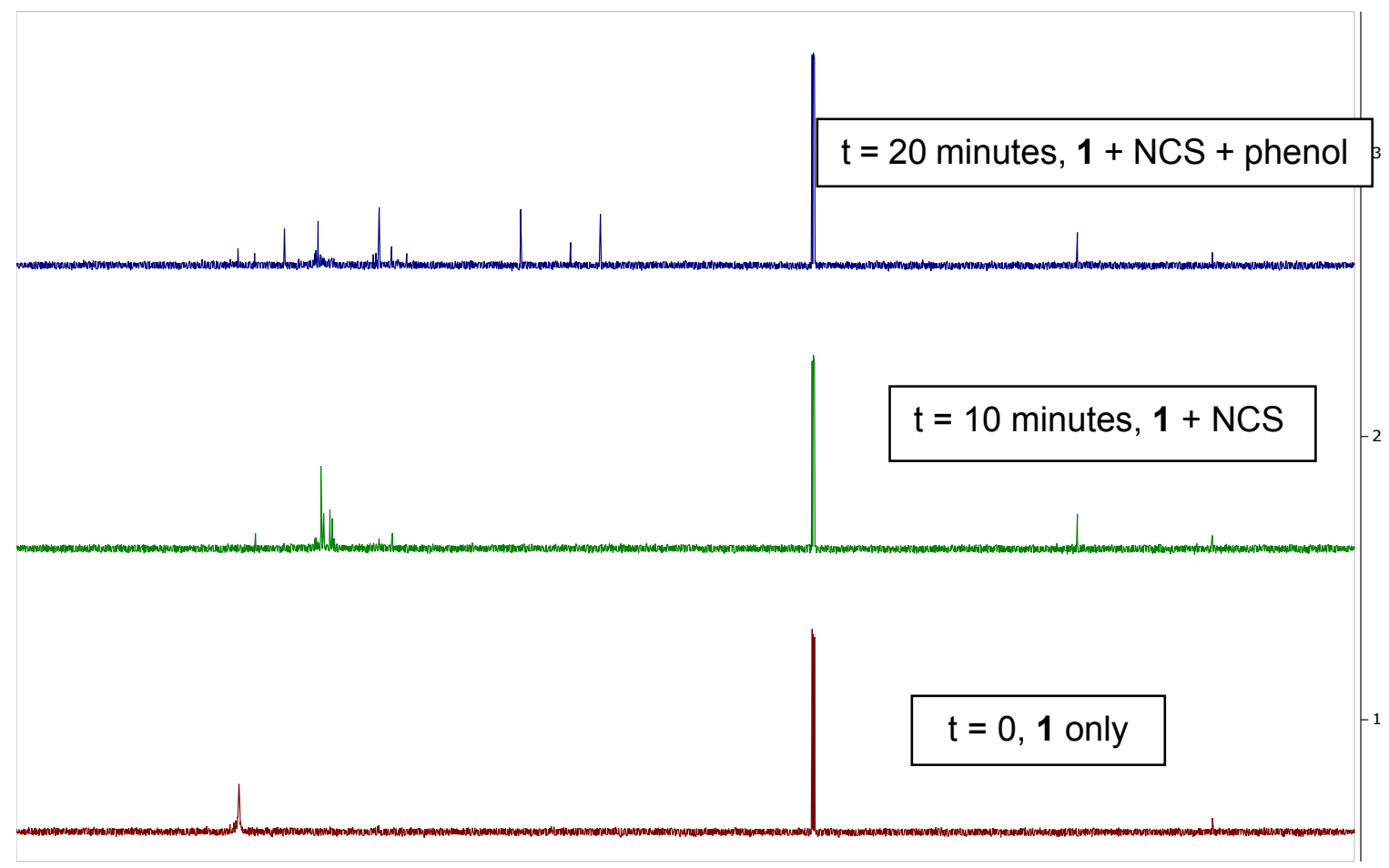

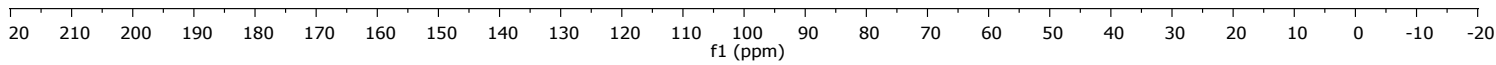

${ }^{15} \mathrm{~N}$ of catalyst 1 Comparison

$\mathrm{t}=\mathbf{2}$ days after phenol

quench

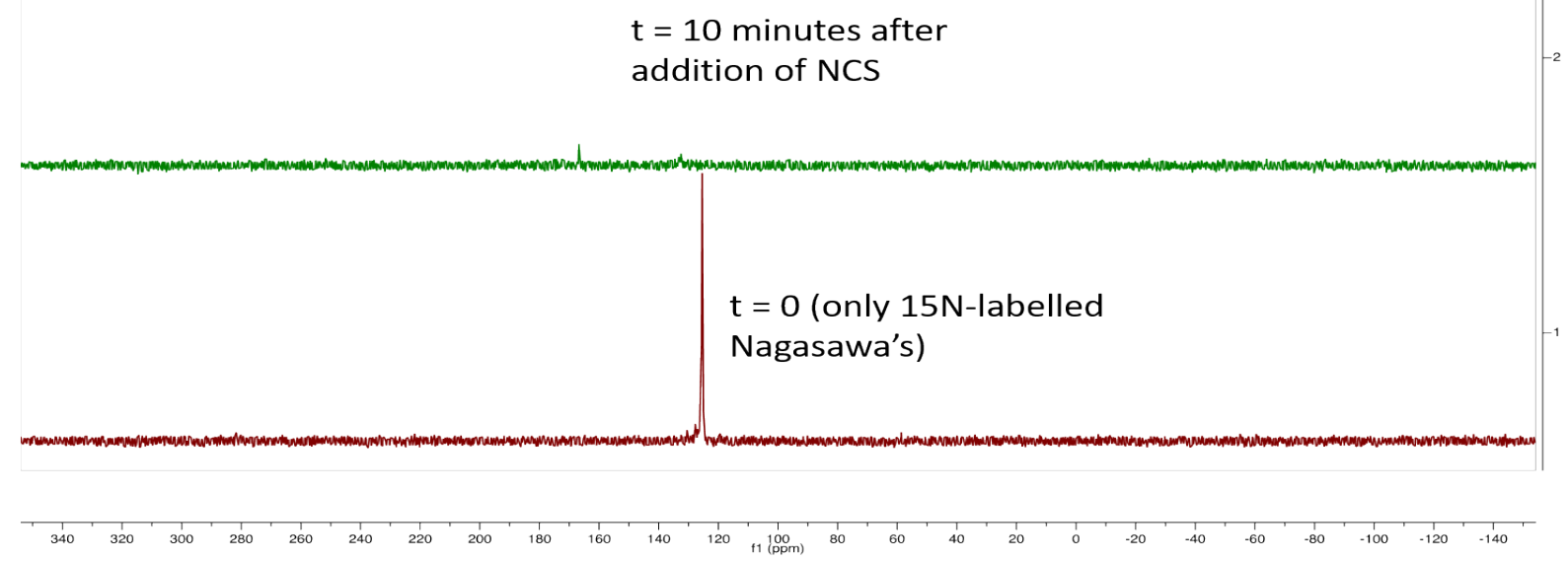


${ }^{15} \mathrm{~N}$ Spectrum of Synthesized 1a (synthesis based on published literature)

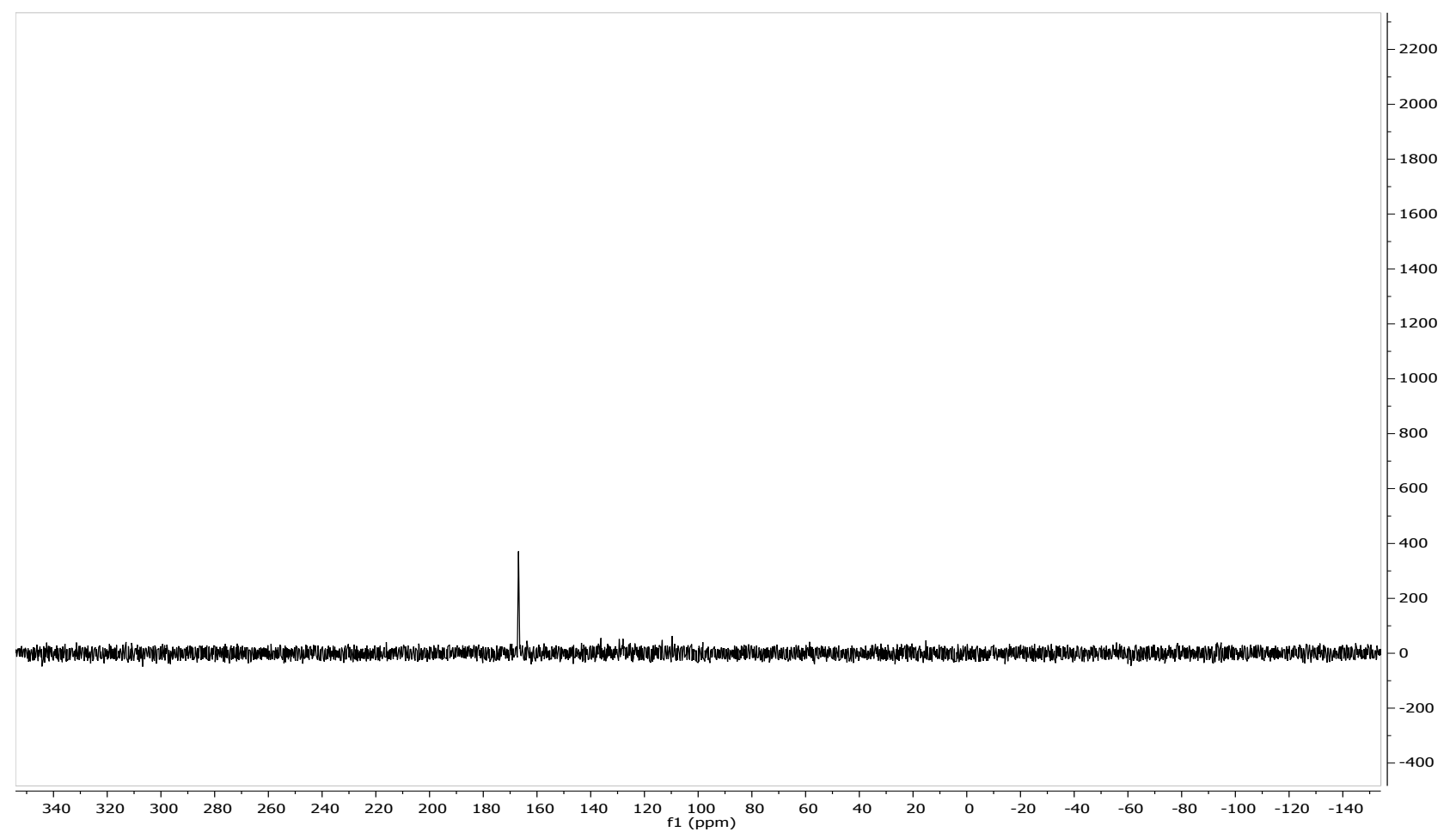


E. Preliminary Mechanistic Experiment: Observing NMR Shifts with Catalyst 10 and Nchlorosuccinimide

$.003 \mathrm{mmol}$ (1 equiv) of catalyst 10 was added to an NMR tube and dissolved in $600 \mu \mathrm{L}$ of $\mathrm{CDCl}_{3}$ $(.005 \mathrm{M})$. The catalyst loading was increased tenfold to obtain better signal on the NMR spectrum. $A{ }^{1} \mathrm{H}$ and ${ }^{19} \mathrm{~F}$ NMR spectrum was taken as $\mathrm{t}=0.8 \mathrm{mg}$ of NCS $(.06 \mathrm{mmol}, 20$ equiv) was added to the reaction NMR tube and stirred. A second ${ }^{1} \mathrm{H}$ and ${ }^{19} \mathrm{~F}$ NMR spectrum was taken as $\mathrm{t}=10 \mathrm{~min}$, showing shifts in the peaks. This observation suggests a coordination occurs between catalyst 10 and $\mathrm{N}$-chlorosuccinimide to form a new intermediate in the catalytic cycle. 
${ }^{1} \mathrm{H}$ and ${ }^{19} \mathrm{~F}$ NMR Spectra of Catalyst 10 . No NCS, $t=0$.

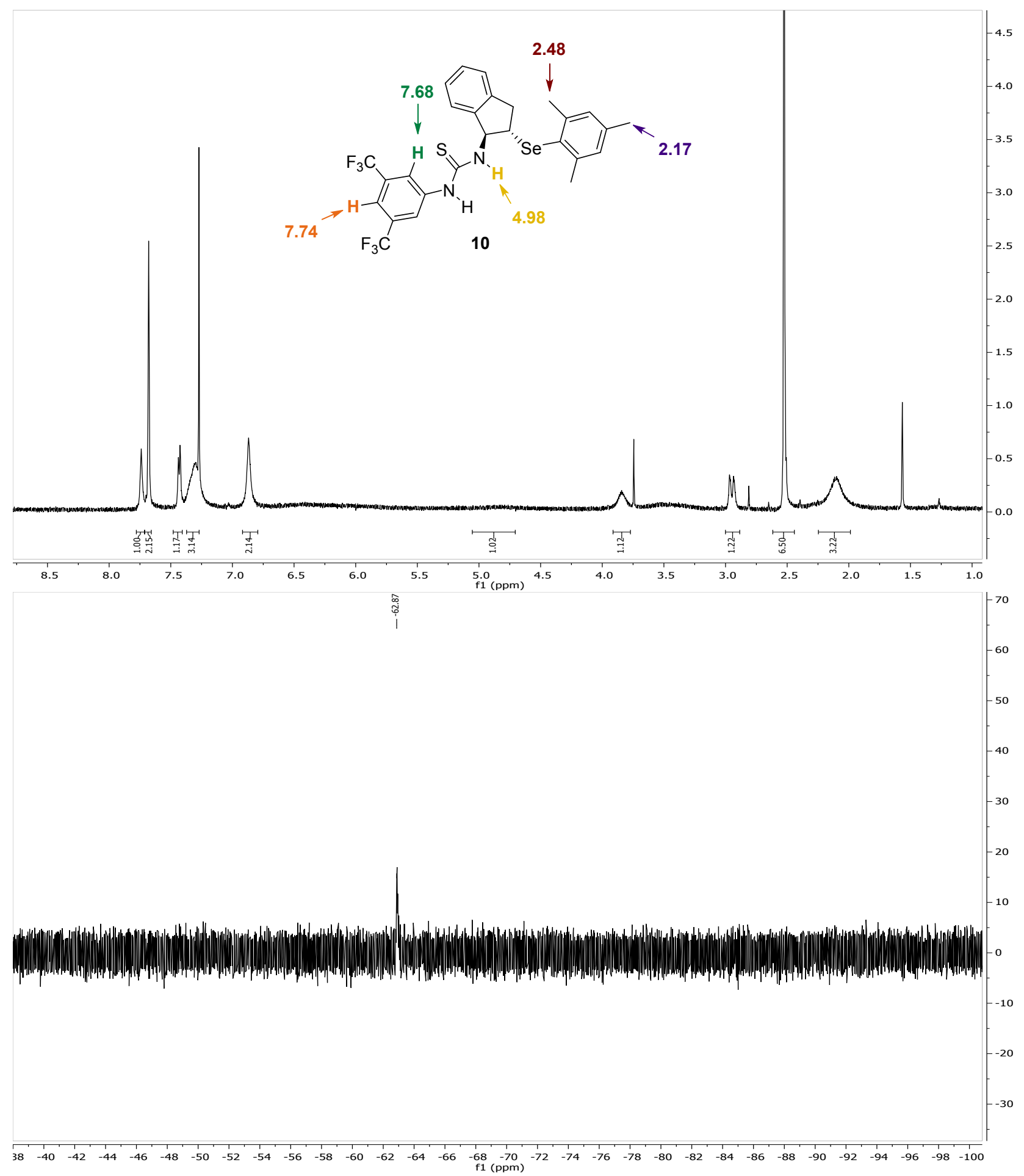


${ }^{1} \mathrm{H}$ and ${ }^{19} \mathrm{~F}$ NMR Spectra of Catalyst 10. NCS added, $t=10$. A possible intermediate is drawn below.
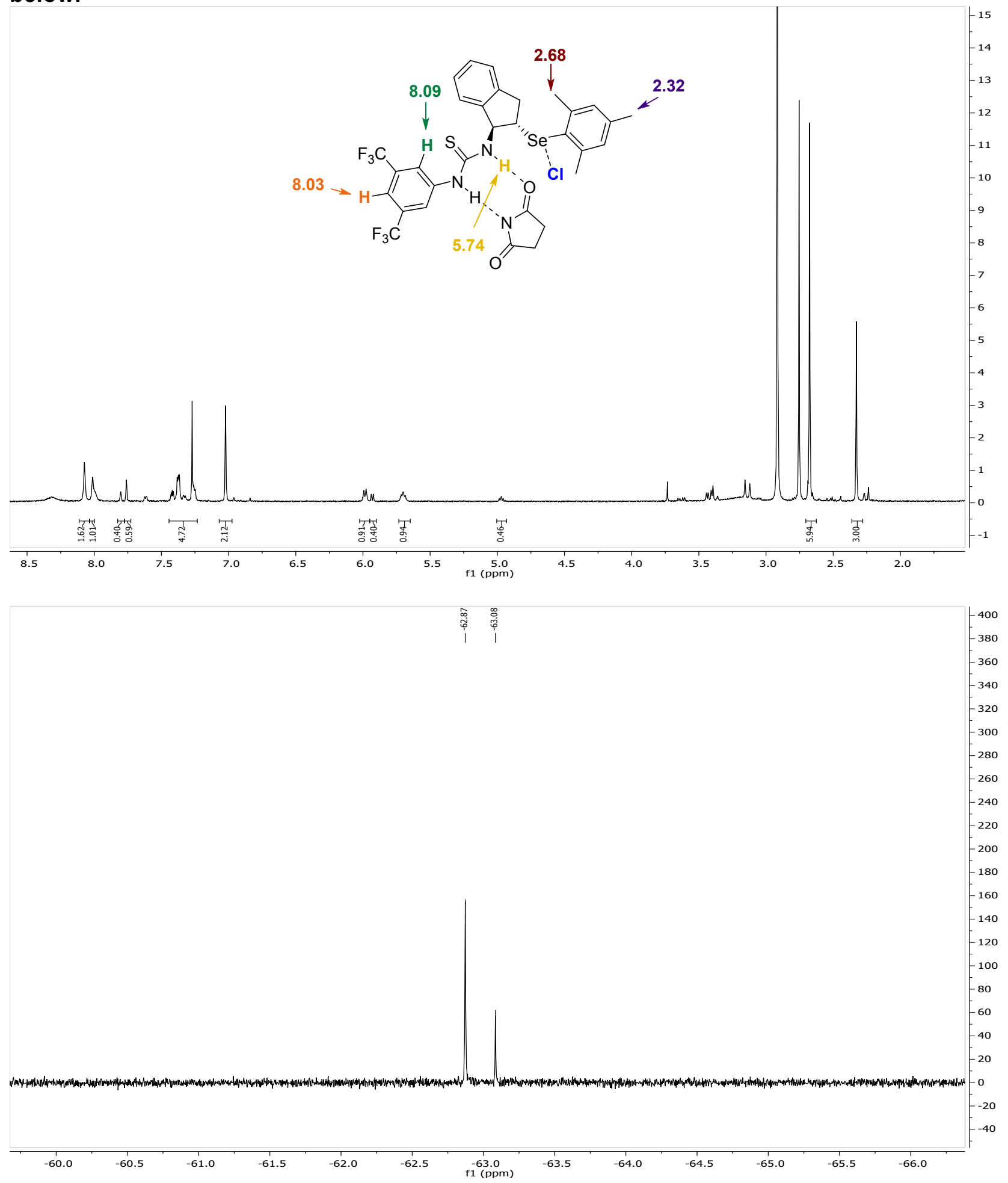
F. Full Optimization Table for ortho chlorination of compound 2a. Note: entry 14 tested the effect of excess succinimide on the conversion and selectivity of the reaction (1.0, 1.5 and 2.0 equivalents were tested). Succinimide was added after the addition of Lewis base catalyst and before the addition of $\mathrm{N}$-chlorosuccinimide. Overall, there is no significant change to either conversion or selectivity. 


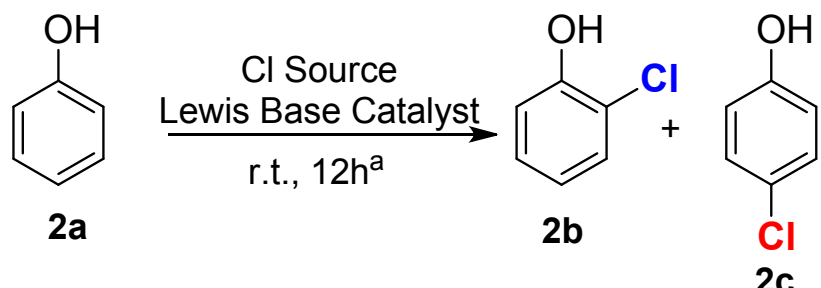

\begin{tabular}{|c|c|c|c|c|c|c|}
\hline Entry & Cat (\%) & Cl Source & Solvent & Conv. $(\%)^{b}$ & $2 b: 2 c^{c}$ & $\Delta \Delta \Delta \mathrm{G}^{\ddagger}(\mathrm{kcal} / \mathrm{mol})^{\mathrm{d}}$ \\
\hline 1 & None & NCS & $\mathrm{CDCl}_{3}$ & 0 & $\mathrm{~N} / \mathrm{A}$ & $\mathrm{N} / \mathrm{A}$ \\
\hline 2 & $\mathrm{PPh}_{3}=\mathrm{S} 3(10 \%)$ & NCS & $\mathrm{CDCl}_{3}$ & 85 & $1.0: 4.0$ & 0.0 \\
\hline 3 & $4(10 \%)$ & NCS & $\mathrm{CDCl}_{3}$ & 40 & $1.0: 3.0$ & 0.17 \\
\hline 4 & $5(10 \%)$ & NCS & $\mathrm{CDCl}_{3}$ & 46 & $1.0: 3.5$ & 0.07 \\
\hline 5 & $6(10 \%)$ & NCS & $\mathrm{CDCl}_{3}$ & 56 & $1.0: 2.7$ & 0.23 \\
\hline 6 & $7(10 \%)$ & NCS & $\mathrm{CDCl}_{3}$ & 51 & $1.5: 1.0$ & 1.05 \\
\hline 7 & $8(10 \%)$ & NCS & $\mathrm{CDCl}_{3}$ & 85 & 1.0:1.0 & 0.81 \\
\hline 8 & $9(10 \%)$ & NCS & $\mathrm{CDCl}_{3}$ & 49 & 2.5:1.0 & 1.35 \\
\hline 9 & $10(10 \%)$ & NCS & $\mathrm{CDCl}_{3}$ & 72 & 4.5:1.0 & 1.71 \\
\hline 10 & $10(10 \%)$ & NCS & DCM & 84 & 3.0:1.0 & 1.47 \\
\hline 11 & $10(10 \%)$ & NCS & Hexane & 56 & 2.8:1.0 & 1.43 \\
\hline 12 & $10(10 \%)$ & NCS & Toluene & 45 & 2.6:1.0 & 1.38 \\
\hline 13 & $10(1 \%)$ & NCS & $\mathrm{CDCl}_{3}$ & 70 & $>20: 1.0$ & 2.59 \\
\hline 14 & $10(1 \%)$ & $\begin{array}{c}\text { XS Succinimide } \\
+ \text { NCS }\end{array}$ & $\mathrm{CDCl}_{3}$ & 68 & $>20: 1.0$ & 2.59 \\
\hline 15 & $10(1 \%)$ & DCDMH & $\mathrm{CDCl}_{3}$ & 90 & 2.0:1.0 & 1.22 \\
\hline
\end{tabular}

Lewis Base Catalyst:<smiles>[R]C1c2ccccc2C[C@H]1Sc1ccccc1</smiles>

4) $R_{1}=N H_{2}$

5) $\mathrm{R}_{1}=\mathrm{NHTs}$

6) $\mathrm{R}_{1}=\mathrm{NHTf}$

7) $R_{1}=$<smiles>CNC(=S)Nc1cc(O)cc(C(F)(F)F)c1</smiles>

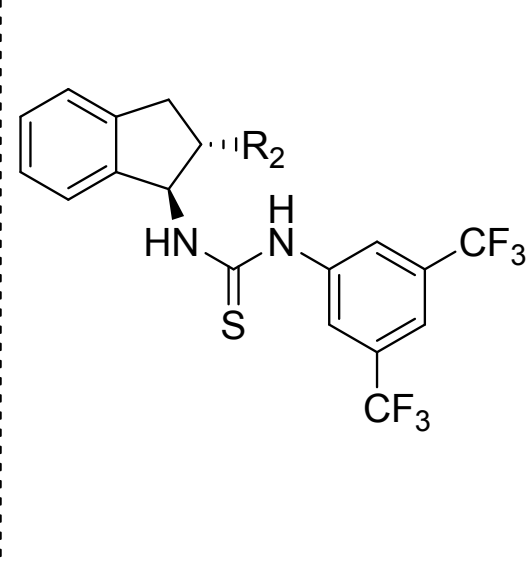




\section{G. Measurement of complete conversion with excess NCS}

The following experiments were done with the exact same procedure written in section A, with the only variation being addition of 1.5 equivalents of NCS instead of 1.2. The experiment was done on a phenol and aniline to show "secondary resolution" and its effect on regioselectivity.

Example ${ }^{1} \mathrm{H} 13 \mathrm{a}+$ catalyst 10 and 1.5 equiv of $\mathrm{NCS} ; \mathrm{t}=12 \mathrm{~h}$

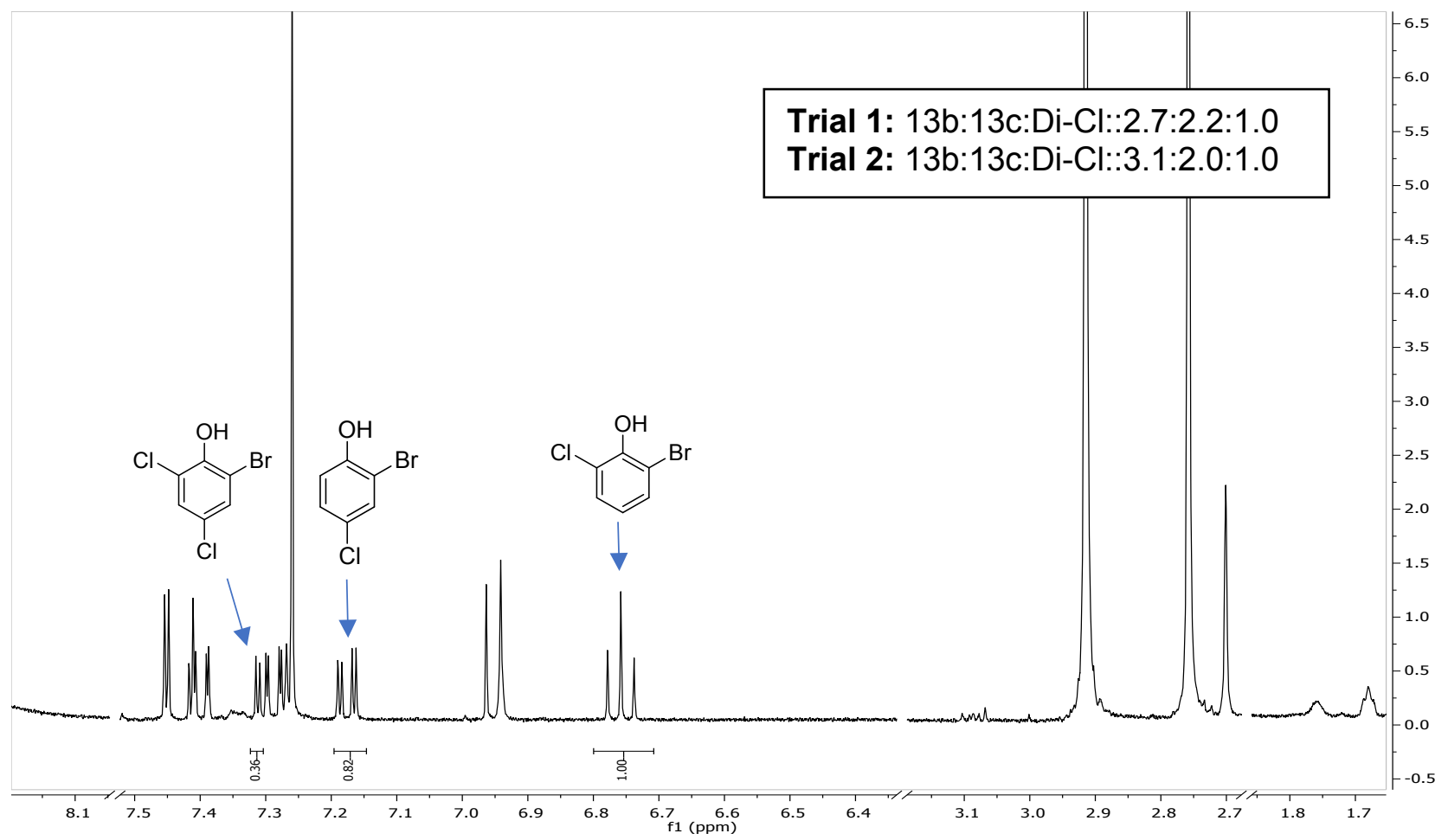


Example ${ }^{1} \mathrm{H} 21 \mathrm{a}+$ catalyst 10 and 1.5 equiv of NCS; $\mathrm{t}=12 \mathrm{~h}$

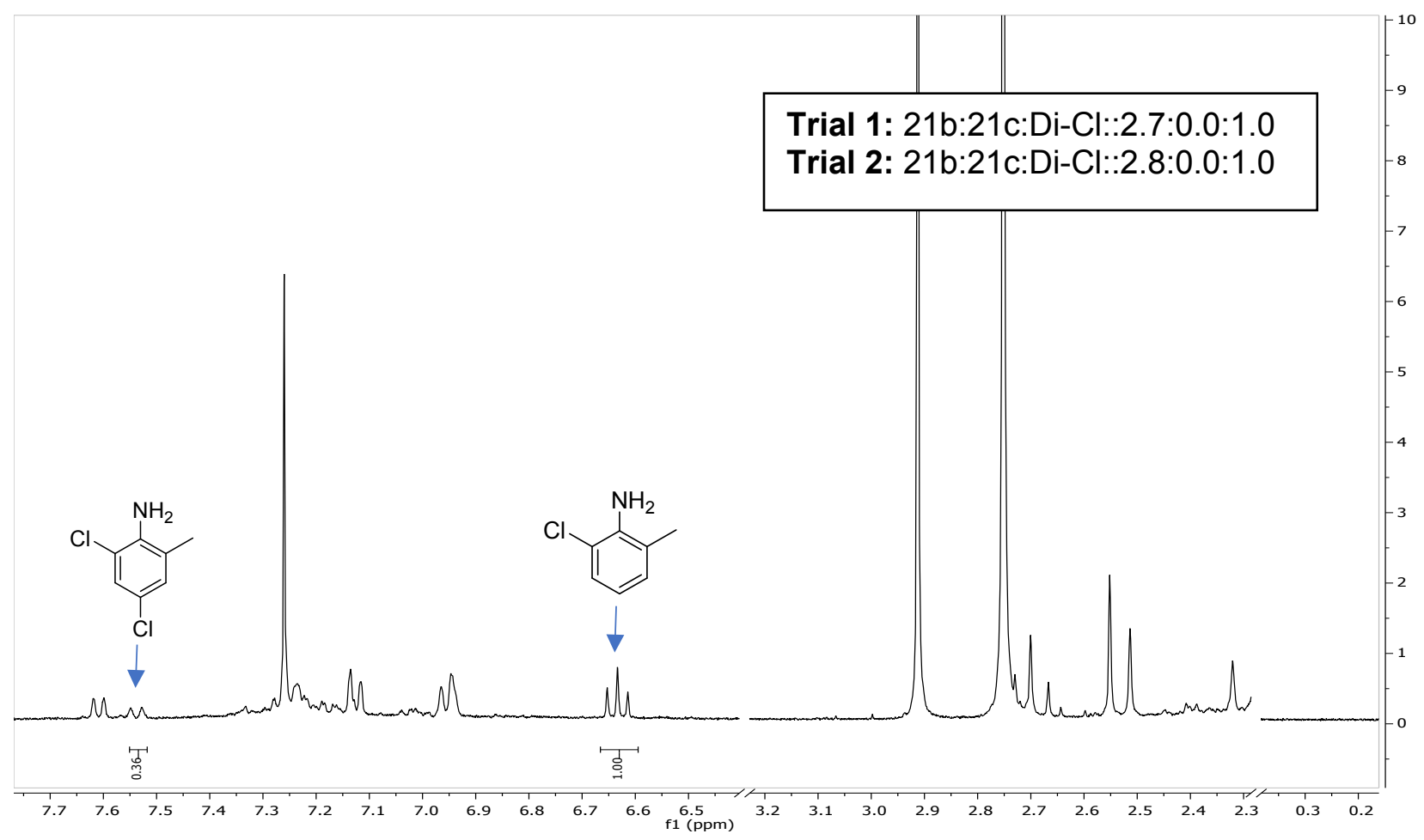

